Arkivoc

Free to Authors and Readers
A Platinum Open Access Journal for Organic Chemistry
Review

DOAJ Seal

Arkivoc 2021, part i, 162-235

\title{
Pyrazole-carboxaldehydes as versatile precursors for different pyrazole- substituted heterocyclic systems
}

\author{
Ismail A. Abdelhamid*, Mahmoud A. E. Hawass, Sherif M. H. Sanad, and Ahmed H. M. Elwahy* \\ Chemistry Department, Faculty of Science, Cairo University, Giza, Egypt \\ Email: ismailshafy@yahoo.com, aelwahy@hotmail.com
}

Received 10-26-2020

Accepted $12-01-2020$

Published on line $02-22-2021$

\section{Abstract}

In the last decade, interest in pyrazole chemistry has grown considerably due to the discovery of fascinating properties demonstrated by a large number of pyrazole derivatives. They occur in a wide range of natural products, dyes, and as scaffolds in a number of drugs and associated pharmaceutical active substances. Substantial attention has been paid to the creation of hybrid molecules in which two heterocycles are bound in a single molecule to enhance their biological effectiveness and overcome drug resistance. In this regard, this review illustrated various methods for the construction of pyrazole-substituted heterocycles and their corresponding fused derivatives using pyrazole carboxaldehydes as effective precursors. The heterocyclic systems mentioned in this review are categorized according to the type of the heterocyclic systems.

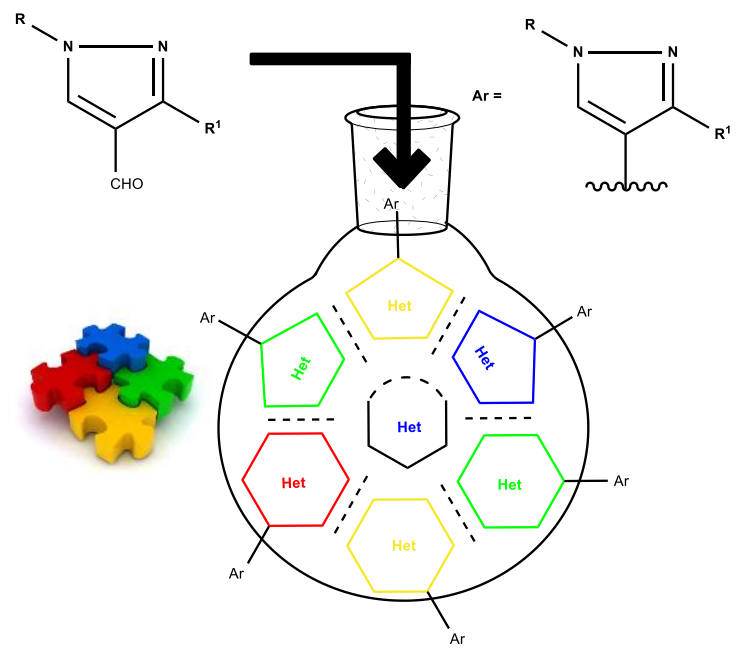

Keywords : Vilsmeier-Haack reaction, pyrazole-carboxaldehydes, pyrazole-substituted heterocycles, pyrazolesubstituted fused-heterocycles 


\section{Table of Contents}

1. Introduction

2. Synthesis of Pyrazole-carboxaldehyde

2.1. Vilsmeier-Haack reaction

2.1.1 Vilsmeier-Haack reaction of hydrazine

2.1.2 Vilsmeier-Haack reaction of pyrazole derivatives

2.1.2.1 Vilsmeier-Haack reaction of pyrazole

2.1.2.2 Vilsmeier-Haack reaction of pyrazolone

2.1.2.3 Vilsmeier-Haack reaction of aminopyrazoles

2.2. Oxidation of the corresponding alcohols

2.3. Reduction of the corresponding pyrazolecarbonitrile

2.4. Hydrolysis of (pyrazolyl)methanimine

2.5. Miscellaneous methods

3. Synthesis of Pyrazole-substituted Heterocycles

3.1. Pyrazole-substituted monoheterocyclic ring

3.1.1 Monocyclic five-membered with one heteroatom

3.1.1.1 Furan derivatives

3.1.1.2 Pyrrole derivatives

3.1.2 Monocyclic five-membered with two heteroatoms

3.1.2.1 Pyrazole derivatives

3.1.2.1.1 Synthesis of pyrazole derivatives from chalcone carrying pyrazole

3.1.2.1.2 Synthesis of pyrazole derivatives from arylidene malononitrile carrying pyrazole

3.1.2.1.3 Synthesis of pyrazole derivatives from cyanoacetohydrazide carrying pyrazole

3.1.2.1.4 Synthesis of pyrazole derivatives from cyanoacrylohydrazide carrying pyrazole

3.1.2.1.5 Miscellaneous methods

3.1.2.2 Imidazole derivatives

3.1.2.3 Oxazole derivatives

3.1.2.4 Isoxazole derivatives

3.1.2.5 Thiazole derivatives

3.1.2.6 1,2-Oxaphosphole derivatives

3.1.3. Monocyclic five-membered with three heteroatoms

3.1.3.1 1,3,4-Thiadiazole derivatives

3.1.3.2 1,2,3-Triazole derivatives

3.1.3.3 1,2,4-Triazole derivatives

3.1.3.4 1,3,4-Oxadiazole derivatives

3.1.4. Monocyclic six-membered with one heteroatom

3.1.4.1 Pyran derivatives

3.1.4.2 Pyridine derivatives

3.1.4.2.1 Synthesis of pyridine derivatives from chalcone carrying pyrazole

3.1.4.2.2 Synthesis of pyridine derivatives from aryliden malononitrile carrying pyrazole

3.1.4.2.3 Synthesis of pyridine derivatives from ethyl arylidencyanoacetate linked to pyrazole moiety

3.1.4.2.4 Synthesis of pyridine derivatives from hydrazone carrying pyrazole 
3.1.4.2.5 Synthesis of pyridine derivatives from reaction of pyrazole-carboxaldehyde with 3aminocrotononitrile

3.1.4.2.6 Synthesis of pyridine derivatives via one-pot reaction of pyrazole-carboxaldehyde

3.1.5. Monocyclic six-membered with two heteroatoms

3.1.5.1. Pyrimidine derivatives

3.1.6. Monocyclic six-membered with three heteroatoms

3.1.6.1. Triazine derivatives

3.1.7. Monocyclic six-membered with four heteroatoms

3.1.7.1. Tetrazine derivatives

3.1.7.2. Oxadiazaphosphinin derivatives

3.2. Pyrazole-substituted fused heterocyclic system

3.2.1. Pyrazole-substituted bicyclic systems

3.2.1.1 Fused [5-6] system with two heteroatoms

3.2.1.1.1 Thiazolo[3,2-a]pyridine derivatives

3.2.1.1.2. Fused [5-6] system with three heteroatoms

3.2.1.1.2.1. Pyrazolo[3,4-b]pyridine derivatives

3.2.1.1.2.2. Thiazolo[3,2-a]pyrimidine derivatives

3.2.1.1.2.3. $1 H$-Imidazo[4,5- $b]$ pyridine derivatives

3.2.1.1.3. Fused [5-6] system with four heteroatoms

3.2.1.1.3.1. $[1,2,4]$ Triazolo[4,3-a]pyrimidine derivatives

3.2.1.1.3.2. Triazolo[ $[1,5-a]$ pyrimidine derivatives

3.2.1.1.3.3. Pyrazolo[3,4- $d]$ pyrimidine derivatives

3.2.1.1.4. Fused [5-6] system with five heteroatoms

3.2.1.1.4.1. 1,2,4-Triazolo[4,3-b]1,2,4-triazine derivatives

3.2.1.1.5. Fused [6-5] system with two heteroatoms

3.2.1.1.5.1. Benzo[d]imidazole derivatives

3.2.1.1.5.2. Benzo[ $[d]$ oxazole derivatives

3.2.1.1.5.3. Benzo[d]thiazole derivatives

3.2.1.1.6. Fused [6-5] system with three heteroatoms

3.2.1.1.6.1. Pyrano[2,3-c]pyrazole derivatives

3.2.1.1.6.2. Pyrano[2,3-d]thiazole derivatives

3.2.1.1.6.3. Thiopyrano[2,3- $d]$ thiazole derivatives

3.2.1.1.7. Fused [6-6] system with one heteroatom

3.2.1.1.7.1. Chromene derivatives

3.2.1.1.1.1.2. Quinoline derivatives

3.2.1.1.7.1.3. Isoquinoline derivatives

3.2.1.1.8. Fused [6-6] system with two heteroatoms

3.2.1.1.8.1 Quinazoline derivatives

3.2.1.1.8.2 Phthalazine derivatives

3.2.1.1.9 Fused [6-6] system with three heteroatoms

3.2.1.1.9.1. Pyridopyrimidine derivatives

3.2.1.1.9.2. Benzo[ $e][1,4,2]$ (ox/ di/ thi)azaphosphinine derivatives

3.2.1.1.10. Fused [6-6] system with four heteroatoms

3.2.1.1.10.1. Pyrimido[4,5- $d]$ pyrimidine derivatives 
3.2.1.1.11. Fused [6-7] system with two heteroatoms

3.2.1.1.11.1. Benzo[b][1,4]thiazepine derivatives

3.2.2. Pyrazole-substituted tricyclic system

3.2.2.1. Fused [5-5-6] system with three heteroatoms

3.2.2.1.1. Cyclopenta[b]pyrazolo[4,3-e]pyridine derivatives

3.2.2.2. Fused [5-5-6] system with five heteroatoms

3.2.2.2.1. Dipyrazolo[3,4-b:4',3'-e]pyridine derivatives

3.2.2.2.2. Dipyrazolo[1,5-a:3',4'-d]pyrimidine derivatives

3.2.2.3. Fused [5-6-5] system with three hetero atoms

3.2.2.3.1. [1,3]Thiazolo[3,2-a]benzimidazole derivatives

3.2.2.3.2. Pyrrolo $\left[3^{\prime}, 4^{\prime}: 5,6\right]$ thiopyrano[2,3- $\left.d\right]$ thiazole derivatives

3.2.2.4. Fused [5-6-6] system with two heteroatoms

3.2.2.4.1. Furo[2,3-f]chromene derivatives

3.2.2.4.2. Pyrazolo[1,2-b]phthalazine derivatives

3.2.2.4.3. Pyrrolo[1,2-a]quinoxalines derivatives

3.2.2.5. Fused [6-5-5] system with five heteroatoms

3.2.2.5.1. Pyrano[2,3-c:6,5-c']dipyrazole derivatives

3.2.2.6. Fused [6-5-6] system with one heteroatom

3.2.2.6.1. Indeno[1,2- $b]$ pyridine derivatives

3.2.2.7. Fused [6-5-6] system with two heteroatom

3.2.2.7.1. Indeno[1,2- $d$ ] pyrimidine derivatives

3.2.2.8. Fused [6-6-6] system with one heteroatom

3.2.2.8.1. Acridine derivatives

3.2.2.8.2. Benzo[ $h]$ chromene and benzo[f]chromene

3.2.2.8.3. Xanthene derivatives

3.2.2.9. Fused [6-6-6] system with two heteroatoms

3.2.2.9.1. Naphtho[1,2-e][1,3]oxazine derivatives

3.2.2.9.2. Pyrano[3,2-c]chromene derivatives

3.2.2.9.3. Chromeno[4,3-b]pyridine derivatives

3.2.2.10. Fused [6-6-6] system with three heteroatoms

3.2.2.10.1. Thiochromeno $[3,4-d]$ pyrimidine derivatives

3.2.2.10.2. Pyrimido[4,5- $b$ ]quinoline derivatives

3.2.2.11. Fused [6-6-6] system with five heteroatoms

3.2.2.11.1. Pyrido[2,3-d:6,5- $\left.d^{\prime}\right]$ dipyrimidine derivatives

3.2.3. Pyrazole-substituted tetracyclic system

3.2.3.1. Fused [6-5-6-6] system with two heteroatoms

3.2.3.1.1. Benzo[ $g]$ thieno[3,4- $b]$ thiochromene derivatives

3.2.3.2. Fused [6-5-6-6] system with four heteroatoms

3.2.3.2.1. Imidazo[4,5-f][1,10]phenanthroline derivatives

3.2.4. Pyrazole-substituted pentacyclic system

3.2.4.1. Fused [6-5-5-6-6] system with nine heteroatoms

3.2.4.1.1. Pyrido[2,3- $\left.d: 6,5-d^{\prime}\right]$ ditriazolopyrimidine derivatives

Conclusions

References 


\section{Introduction}

Heterocycles are significant classes of compounds that make up more than half of all known organic compounds. They exist in a wide range of medications, most supplements, many natural products, and biomolecules like hormones, antibiotics, alkaloids, vitamins, etc. The vast majority of commercially available synthetic drugs have a heterocyclic structural component. Many heterocyclic compounds were found to exhibit a wide variety of biological activities including antitumor, antibiotics, anti-inflammatory, antidepressant, antimalarial, anti-HIV, antimicrobial, antibacterial, antiviral, antidiabetic, herbicide, and fungicide agents. Many of the heterocycles have also many applications such as dyestuff, fluorescent sensor, brightening agents, information storage, plastics, and analytical reagents. Heterocycles are also of great interest as intermediates, protecting groups, chiral auxiliaries, organic catalysts, and metal ligands. In addition, ionic liquids composed of heterocyclic compounds can serve as green solvents as well as catalyst. ${ }^{1-20}$

Nitrogen-containing heterocycles are among the most active compounds due to their large occurrence in natural products. They are among the core structures of various biologically active compounds and are considered as essential roles in many of the chemical reactions occurring in all organisms. ${ }^{19}$ They also show numerous applications in chemistry, biology, and other sciences. In addition, nitrogen-containing heterocycles play a significant role in coordination chemistry. ${ }^{21}$

Among different nitrogen-containing heterocycles, pyrazole derivatives represent an interesting class of fivemembered heterocycles. ${ }^{11}$ Pyrazole is a motif found in a number of molecules that have a wide range of agricultural and pharmaceutical activities. ${ }^{12,22}$

Pyrazole derivatives exhibited a wide variety of biological profiles, such as anti-tuberculosis, anti-AIDS, anti-malarial, anti-microbial, antitumor, antifungal, anti-hyperglycemic agents, anti-depressant agents, anticonvulsant agents, antipyretic agents, and anti-anxiety agents. ${ }^{23-29}$ The pyrazole ring is involved in diverse therapeutic active compounds. In this respect, a variety of well-known drugs belonging to various categories such as celecoxib, rimonabant, fomepizole, and sildenafil have been recently developed.

Some of the pyrazole derivatives have important applications as brightening agents ${ }^{30}$ and some exhibit significant solvatochromic and electroluminescence properties. ${ }^{31}$ Their application in material chemistry, ${ }^{32}$ semiconductors, ${ }^{33}$ liquid crystals, ${ }^{34}$ and organic light-emitting diodes ${ }^{35}$ have been extensively reported.

The diversity in the numerous potential applications of pyrazoles encouraged the continuous investigation of this class of compounds and prompted authors to search for more effective and selective synthetic routes to this type of compounds and allowing the production of a large number of structurally diverse derivatives with various biological profiles.

Continuing our interest in reviewing various approaches to heterocyclic system synthesis, ${ }^{36-51}$ this review highlights the different synthetic methods for the preparation of pyrazole-carboxaldehydes and their usefulness as versatile precursors for different pyrazole-substituted heterocyclic systems. Based on the size of the heterocyclic ring as well as the position and number of the heteroatoms, heterocyclic compounds mentioned in this review are arranged.

\section{Synthesis of Pyrazole-carboxaldehyde}

There have been several important routes to synthesize pyrazole-carboxaldehydes, e.g. (i) Vilsmeier-Haack reaction of hydrazones, (ii) Oxidation of the corresponding alcohols, (iii) Reduction of the corresponding nitrile and (iv) Miscellaneous methods. 


\subsection{Vilsmeier-Haack reaction}

2.1.1 Vilsmeier-Haack reaction of hydrazine. This method is the most common one to synthesize pyrazole-4carboxaldehydes 2 via the corresponding hydrazonoyl derivatives 1 (Scheme 1, Table 1).

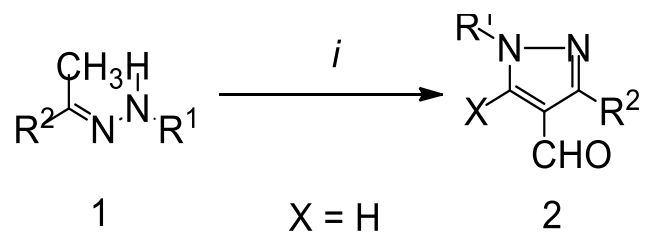

$i=N, N$-dimethyl formamide (DMF)/ phosphorus oxychloride $\left(\mathrm{POCl}_{3}\right) /$ heat; ${ }^{52-78}$ 2,4,6-trichloro[1,3,5]triazine (TCT)/DMF /r.t/ $\mathrm{Na}_{2} \mathrm{CO}_{3}{ }^{79}$

Scheme 1. Synthesis of pyrazole-carboxaldehydes by Vilsmeier-Haack reaction of hydrazones.

Phenylsulfonyl- $N, N$-dimethylformimidamide-pyrazole-4-carboxaldehydes 4 were obtained by VilsmeierHaack reaction of the corresponding benzenesulfonamide hydrazonoyl derivatives 3 with $\mathrm{POCl}_{3}$ in $\mathrm{DMF}$ (Scheme 2). 80,81

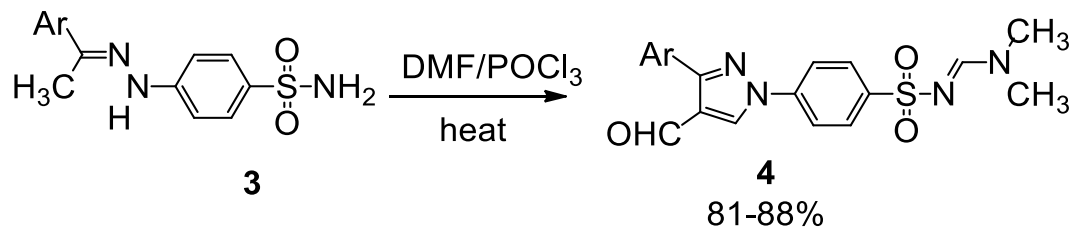

$\mathrm{Ar}=\mathrm{C}_{6} \mathrm{H}_{5}, 4-\mathrm{H}_{3} \mathrm{C}-\mathrm{C}_{6} \mathrm{H}_{4}, 4-\mathrm{Br}-\mathrm{C}_{6} \mathrm{H}_{4}, 4-\mathrm{Cl}-\mathrm{C}_{6} \mathrm{H}_{4}, 4-\mathrm{O}_{2} \mathrm{~N}-\mathrm{C}_{6} \mathrm{H}_{4}, 4-\mathrm{H}_{3} \mathrm{CO}-\mathrm{C}_{6} \mathrm{H}_{4}, 4-\mathrm{F}-\mathrm{C}_{6} \mathrm{H}_{4}, 2-$ Thiophene

Scheme 2. Synthesis of phenylsulfonyl-N,N-dimethylformimidamide-pyrazole-4-carboxaldehydes 4.

Table 1. Yields (\%) of compounds 2 prepared from hydrazones

\begin{tabular}{|c|c|c|c|c|}
\hline NO. & $\mathrm{R}^{1}$ & $\mathrm{R}^{2}$ & Yield\% & Ref. \\
\hline 1 & $\mathrm{C}_{6} \mathrm{H}_{5}$ & 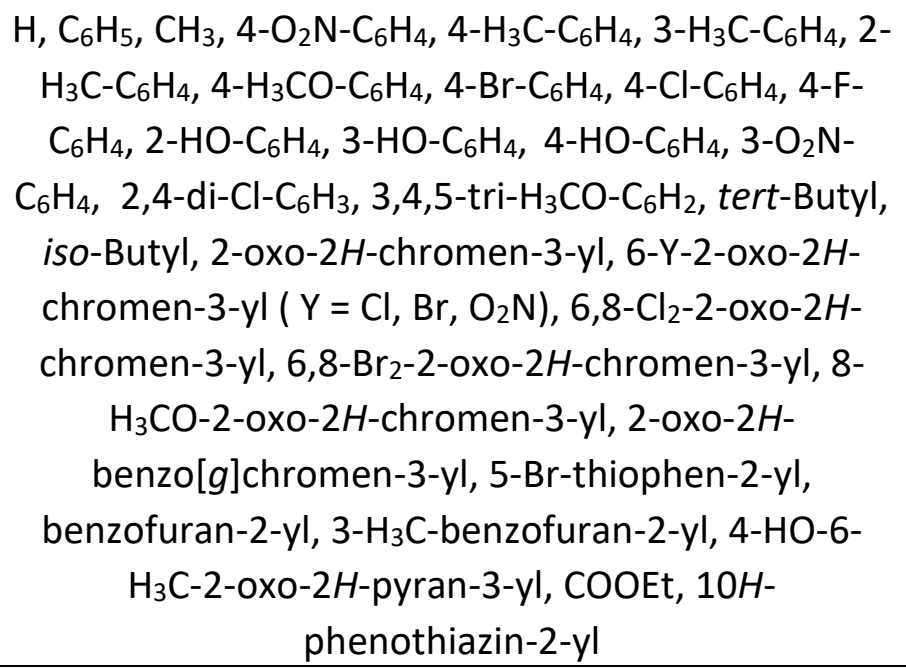 & $38-95$ & $\begin{array}{l}52,53,62-66,72- \\
76,54,77-79,55-61\end{array}$ \\
\hline 2 & $4-\mathrm{O}_{2} \mathrm{~N}-\mathrm{C}_{6} \mathrm{H}_{4}$ & $\begin{array}{l}\mathrm{C}_{6} \mathrm{H}_{5} \text {, Benzofuran-2-yl, 3- } \mathrm{H}_{3} \mathrm{C}-\text { benzofuran-2-yl, 4- } \\
\text { HO-6- } \mathrm{H}_{3} \mathrm{C}-2-\text { oxo-2H-pyran-3-yl, Benzofuran-2-yl, 3- }\end{array}$ & $58-94$ & $52,63,75,76$ \\
\hline
\end{tabular}


$\mathrm{H}_{3} \mathrm{C}$-benzofuran-2-yl, 4-HO-6- $\mathrm{H}_{3} \mathrm{C}-2-\mathrm{oxo}-2 \mathrm{H}$-pyran-

$3-\mathrm{yl}$

Table 1. Continued

\begin{tabular}{|c|c|c|c|c|}
\hline NO. & $\mathrm{R}^{1}$ & $\mathrm{R}^{2}$ & Yield\% & Ref. \\
\hline 3 & $4-\mathrm{Cl}-\mathrm{C}_{6} \mathrm{H}_{4}$ & $\begin{array}{c}\mathrm{H}, \mathrm{C}_{6} \mathrm{H}_{5}, 4-\mathrm{H}_{3} \mathrm{C}-\mathrm{C}_{6} \mathrm{H}_{4}, 4-\mathrm{Cl}-\mathrm{C}_{6} \mathrm{H}_{4}, 4-\mathrm{H}_{3} \mathrm{CO}-\mathrm{C}_{6} \mathrm{H}_{4}, 4-\mathrm{Br}- \\
\mathrm{C}_{6} \mathrm{H}_{4}, 4-\mathrm{F}-\mathrm{C}_{6} \mathrm{H}_{4}, 4-\mathrm{HO}-6-\mathrm{H}_{3} \mathrm{C}-2-\text { oxo-2 } \\
\text { COO-pyran-3-yl, }\end{array}$ & $47-92$ & $53,61,66,67,76$ \\
\hline 4 & $4-\mathrm{H}_{3} \mathrm{C}-\mathrm{C}_{6} \mathrm{H}_{4}$ & $\mathrm{H}, 4-\mathrm{OH}-6-\mathrm{H}_{3} \mathrm{C}-2-0 x 0-2 \mathrm{H}$-pyran-3-yl, $\mathrm{C}_{6} \mathrm{H}_{5}$ & $42-80$ & $61,76,79$ \\
\hline 5 & $\mathrm{H}$ & 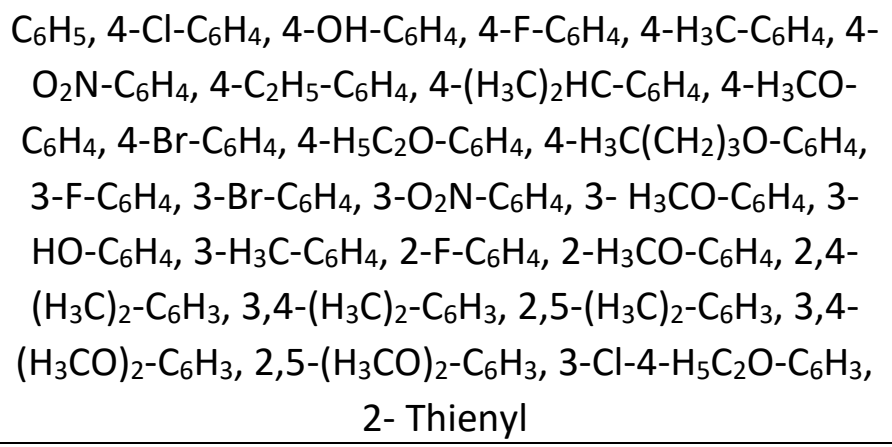 & $32-87$ & 68 \\
\hline 6 & $\begin{array}{c}2,4-\left(\mathrm{O}_{2} \mathrm{~N}\right)_{2^{-}} \\
\mathrm{C}_{6} \mathrm{H}_{3}\end{array}$ & 4-OH-6- $\mathrm{H}_{3} \mathrm{C}-2-\mathrm{oxo}-2 \mathrm{H}$-pyran-3-yl, $\mathrm{CO}_{2} \mathrm{Et}$ & 55 & 69,76 \\
\hline 7 & isonicotinoyl & $4-\mathrm{NO}_{2}-\mathrm{C}_{6} \mathrm{H}_{4}$ & 60 & 70 \\
\hline 8 & $\begin{array}{c}2,6-\mathrm{Cl}_{2}-4- \\
\mathrm{F}_{3} \mathrm{C}-\mathrm{C}_{6} \mathrm{H}_{2}\end{array}$ & $\begin{array}{c}\mathrm{C}_{6} \mathrm{H}_{5}, 4-\mathrm{Cl}-\mathrm{C}_{6} \mathrm{H}_{4}, 3-\mathrm{Cl}-\mathrm{C}_{6} \mathrm{H}_{4}, 4-\mathrm{Br}-\mathrm{C}_{6} \mathrm{H}_{4}, 3-\mathrm{Br}^{-} \mathrm{C}_{6} \mathrm{H}_{4}, 4- \\
\mathrm{H}_{3} \mathrm{CO}-\mathrm{C}_{6} \mathrm{H}_{4}, 4-\mathrm{F}_{3} \mathrm{C}-\mathrm{C}_{6} \mathrm{H}_{4}, 4-\mathrm{O}_{2} \mathrm{~N}-\mathrm{C}_{6} \mathrm{H}_{4}, 6-\mathrm{H}_{3} \mathrm{CO}- \\
\text { naphthalen-2-yl }\end{array}$ & $81-89$ & 71 \\
\hline 9 & $3-\mathrm{H}_{3} \mathrm{C}-\mathrm{C}_{6} \mathrm{H}_{4}$ & COOEt & 73 & 66 \\
\hline 10 & $4-\mathrm{F}-\mathrm{C}_{6} \mathrm{H}_{4}$ & $\mathrm{C}_{6} \mathrm{H}_{5}$ & 82 & 79 \\
\hline
\end{tabular}

Ramu and Rajagopal ${ }^{82}$ reported that the Vilsmeier reaction of the bis-acetyl carbazole hydrazones $\mathbf{5}$ yielded the corresponding 3,3'-(9-alkyl-carbazole-3,6-diyl)bis(1-phenyl-1H-pyrazole-4-carboxaldehyde) 6 in good yield (Scheme 3).
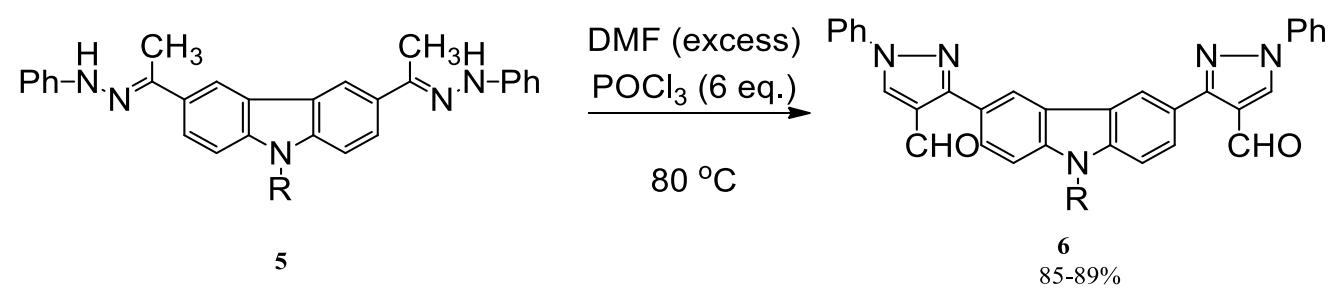

$\mathrm{R}=\mathrm{CH}_{3}, \mathrm{C}_{2} \mathrm{H}_{5}, n-\mathrm{C}_{4} \mathrm{H}_{9}, \mathrm{H}_{2} \mathrm{CC}_{6} \mathrm{H}_{5}$

Scheme 3. Synthesis of 3,3'-(9-alkyl-carbazole-3,6-diyl)bis(1-phenyl-1H-pyrazole-4-carboxaldehyde) 6.

2.1.2. Vilsmeier-Haack reaction of pyrazole derivatives. 2.1.2.1. Vilsmeier-Haack reaction of pyrazole. Heating of pyrazole 7 with DMF/POCl 3 gave the corresponding pyrazole-carboxaldehyde $\mathbf{2}$ (Scheme 4, Table 2). ${ }^{76,83-85}$ 


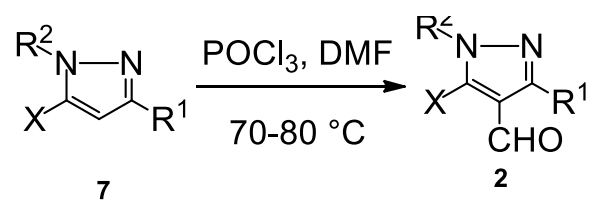

Scheme 4. Synthesis of pyrazole-carboxaldehyde 2 from pyrazole 7.

Table 2. Yields (\%) of compounds 2 prepared from pyrazoles

\begin{tabular}{cccccc}
\hline $\mathrm{NO}$ & $\mathrm{R}^{1}$ & $\mathrm{R}^{2}$ & $\mathrm{X}$ & Yield\% & Ref. \\
\hline 1 & $\mathrm{BnO}$ & $\mathrm{C}_{6} \mathrm{H}_{5}$ & $\mathrm{H}$ & 60 & 83 \\
2 & $\mathrm{CH}_{3}$ & $\mathrm{C}_{6} \mathrm{H}_{5}$ & $\mathrm{Cl}$ & 85 & 84 \\
3 & $4-\mathrm{HO}-6-\mathrm{H}_{3} \mathrm{C}-2-$ & $\mathrm{C}_{6} \mathrm{H}_{5}, 4-\mathrm{H}_{3} \mathrm{C}-\mathrm{C}_{6} \mathrm{H}_{4}, 4-\mathrm{Cl}_{-}-\mathrm{C}_{6} \mathrm{H}_{4}, 4-$ & $\mathrm{H}$ & 50 & 76 \\
& $0 \times 0-2 \mathrm{H}-\mathrm{pyran}-3-$ & $\mathrm{O}_{2} \mathrm{~N}-\mathrm{C}_{6} \mathrm{H}_{4}, 2,4-\left(\mathrm{O}_{2} \mathrm{~N}\right)_{2}-\mathrm{C}_{6} \mathrm{H}_{3}$ & & & \\
& $\mathrm{yl}$ & $\mathrm{C}_{2} \mathrm{H}_{5}$ & & \\
5 & $\mathrm{H}$ & $\mathrm{CH}_{3} \mathrm{C}_{2} \mathrm{H}_{5}, \mathrm{C}_{3} \mathrm{H}_{7}$ & $\mathrm{H}$ & 77 & 85 \\
5 & $\mathrm{CH}_{3}$ & $\mathrm{CH}_{3}$ & 69 & 85,86 \\
\hline
\end{tabular}

In contrast, 3,5-dimethyl-1H-pyrazole 8 did not undergo formylation at position 4 under analogous conditions. However, the protection of compound $\mathbf{8}$ through its reaction with methyl acrylate $\mathbf{9}$ affords methyl3-(3,5-dimethyl-1H-pyrazol-1-yl)propanoate 10. Subsequent reaction of 10 with $\mathrm{POCl}_{3} / \mathrm{DMF}$ afforded methyl 3(4-formyl-3,5-dimethyl-1H-pyrazol-1-yl)propionate 11 which undergo alkaline hydrolysis to give methyl 3-(4formyl-3,5-dimethyl-1H-pyrazol-1-yl)propanoate 12 . Subsequent heating of 12 at $250{ }^{\circ} \mathrm{C}$ gave 3,5 -dimethyl- $1 H$ pyrazole-4-carboxaldehyde 13 (Scheme 5). ${ }^{86}$

2-(Pyrazol-1-yl)-ethanoles 14 do not undergo Vilsmeier-Haack formylation and instead $N$ chloroethylpyrazoles 15 were formed. The reaction of $N$-chloroethylpyrazole 15 with Vilsmeier reagent gave $N$ chloroethylpyrazole-4-carboxaldehyde 16 in 8-10\% yield. On the other hand, synthesis of 1-(2-hydroxyethyl)3,5-dimethyl-1H-pyrazole-4-carboxaldehyde 17 took place by acylation of 14 with acetic anhydride or vinyl acetate in the presence of a catalytic amount of copper acetate to give acylated products 17 which readily underwent Vilsmeier-Haack formylation to give 18. Subsequent hydrolysis of 18 afforded 19 which underwent chlorination to give 16 upon treatment of Vilsmeier reagent (Scheme 6).$^{87,88}$

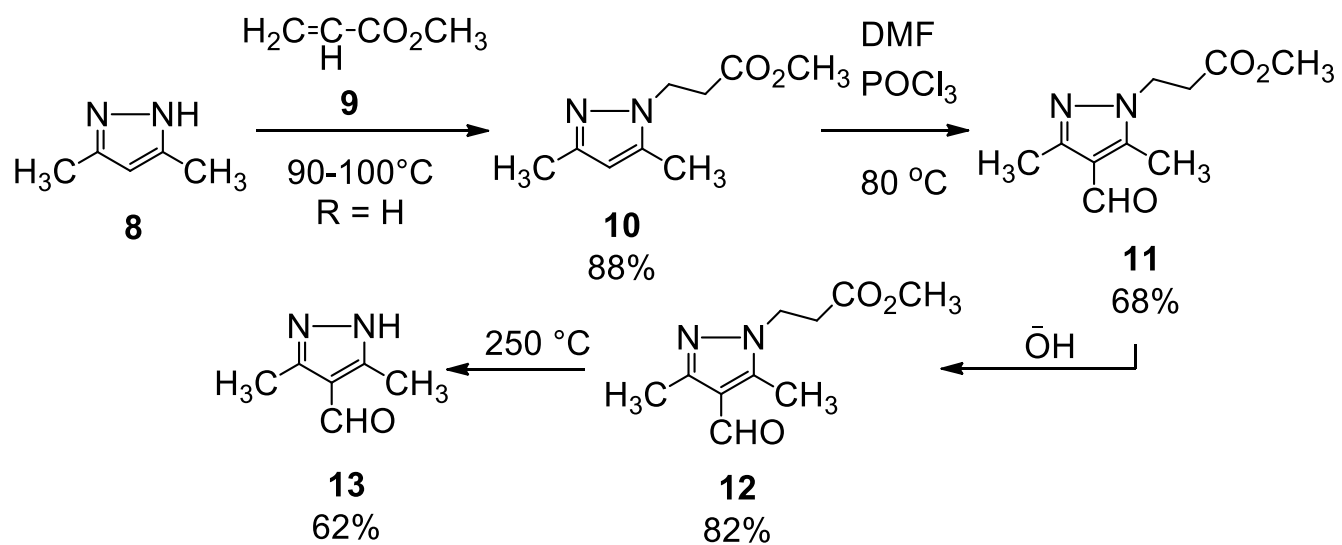


Scheme 5. Synthesis of 3,5-dimethyl-1H-pyrazole-4-carboxaldehyde 13.

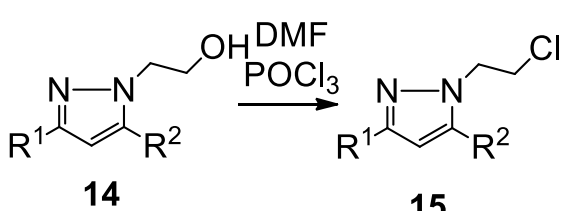

14

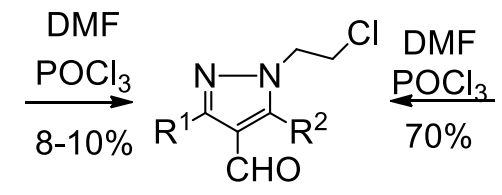

16<smiles>[R]c1nn(CCO)c([R])c1C</smiles>

19

$57-75 \%$

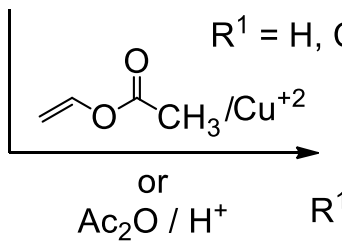

$\mathrm{H}, \mathrm{CH}_{3} ; \mathrm{R}^{2}=\mathrm{H}, \mathrm{CH}_{3}$

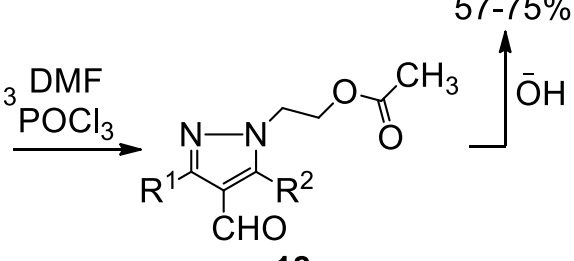

17

18

Scheme 6. Synthesis of 2-(4-formyl-1H-pyrazol-1-yl)ethyl acetate.

Vilsmeier-Haack reaction of bis(3,5-dimethyl-1H-pyrazol-1-yl)methane 19 afforded 1,1'Methylenebis(3,5-dimethylpyrazole-4-carboxaldehyde) 20 (Scheme 7)..$^{85}$

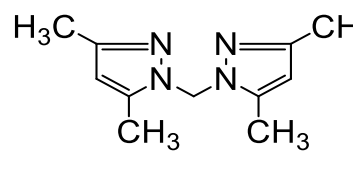

19

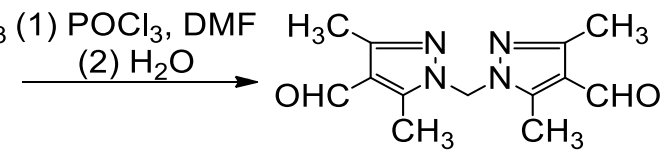

20

$87 \%$

Scheme 7. Synthesis of 1,1'-Methylenebis(3,5-dimethylpyrazole-4-carboxaldehyde) 20.

2.1.2. Vilsmeier-Haack reaction of pyrazolone. Wallace and Straley ${ }^{89}$ reported the synthesis of 3-methyl-5oxo-1-phenyl-2-pyrazoline-4-carboxaldehyde $\mathbf{2 2}$ in good yield by treating the pyrazolinone $\mathbf{2 1}$ with DMF and $\mathrm{POCl}_{3}$ (Scheme 8).

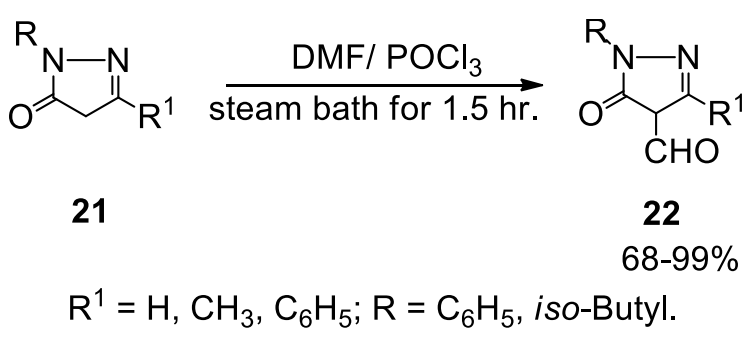

Scheme 8. Synthesis of 3-methyl-5-oxo-1-phenyl-2-pyrazoline-4-carboxaldehyde 22.

However, it was reported by others, that pyrazol-5-ones $\mathbf{2 1}$ underwent formylation using VilsmeierHaack conditions to give the corresponding 5-chloropyrazole-4-carboxaldehydes 2 (Scheme 9, Table 3). ${ }^{90-93}$ 


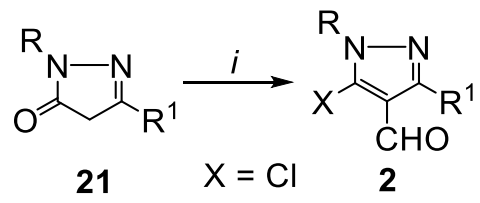

Scheme 9. Synthesis of 5-chloropyrazole-4-carboxaldehydes 2.

Table 3. Yields (\%) of compounds $\mathbf{2}$ prepared from pyrazolones $\mathbf{2 1}$

\begin{tabular}{|c|c|c|c|c|c|}
\hline Entry & $\mathrm{R}$ & $\mathrm{R}^{1}$ & $i$ & Yield\% & Ref. \\
\hline 1 & $\mathrm{CH}_{3}$ & $\mathrm{CH}_{3}, \mathrm{C}_{6} \mathrm{H}_{5}$ & 1) $\mathrm{DMF} / \mathrm{POCl}_{3} \quad$ 2) $\mathrm{POCl}_{3}$ & 94 & 90 \\
\hline 2 & $\mathrm{C}_{6} \mathrm{H}_{5}$ & $\begin{array}{c}4-\mathrm{H}_{3} \mathrm{C}-\mathrm{C}_{6} \mathrm{H}_{4}, \mathrm{CH}_{3}, \mathrm{C}_{6} \mathrm{H}_{5}, 4- \\
\mathrm{F}-\mathrm{C}_{6} \mathrm{H}_{4}, 4-\mathrm{Cl}-\mathrm{C}_{6} \mathrm{H}_{4}, 4-\mathrm{H}_{3} \mathrm{CO}- \\
\mathrm{C}_{6} \mathrm{H}_{4}\end{array}$ & $\begin{array}{c}\mathrm{BTC}(\mathrm{Bis} \text { (trichloromethyl) } \\
\text { carbonate) / DMF/ Chlorobenzene, } \\
130{ }^{\circ} \mathrm{C}\end{array}$ & $57-86$ & 91 \\
\hline 3 & $\mathrm{C}_{6} \mathrm{H}_{5}$ & $\mathrm{C}_{6} \mathrm{H}_{5}$, Pyridyl & $\mathrm{DMF} / \mathrm{POCl}_{3} /$ heat & $60-75$ & 92,93 \\
\hline
\end{tabular}

2.1.2.3. Vilsmeier-Haack reaction of aminopyrazoles. Vilsmeier-Haack formylation of 5 -aminopyrazoles 23 with excess $\mathrm{DMF} / \mathrm{POCl}_{3}$ under conventional heating ${ }^{94}$ or $\mathrm{MW}$ irradiation ${ }^{95}$ led to the formation of 4-formylpyrazolyl-dimethylimidoformamides 24 (Scheme 10, Table 4). ${ }^{94,95}$

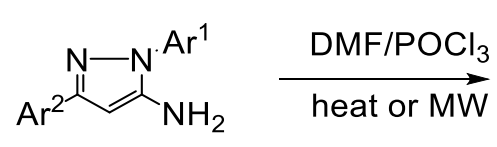

23<smiles></smiles>

24

Scheme 10. Synthesis of 4-formyl-pyrazolyl-dimethylimidoformamides 24.

$N$ - $N$-Disubstituted- $N$-[1,3-diphenyl-4-formyl-1H-pyrazol-5-yl] formimidamides 25 were synthesized by microwave irradiation of 5-amino-1,3-diphenyl-1H-pyrazole $\mathbf{2 3}$ with various amide solvents in the presence of $\mathrm{POCl}_{3}$ (Scheme 11, Table 5). ${ }^{95}$

Table 4. Yields (\%) of compounds 24

\begin{tabular}{|c|c|c|c|c|c|}
\hline Entry & $A r^{1}$ & $A r^{2}$ & Cond. & Yield\% & Ref. \\
\hline 1 & $\mathrm{C}_{6} \mathrm{H}_{5}$ & $\begin{array}{c}4-\mathrm{Cl}-\mathrm{C}_{6} \mathrm{H}_{4}, 4-\mathrm{Br}-\mathrm{C}_{6} \mathrm{H}_{4}, 4-\mathrm{H}_{3} \mathrm{C}- \\
\mathrm{C}_{6} \mathrm{H}_{4}\end{array}$ & heat & $72-78$ & 94 \\
\hline 2 & $\begin{array}{c}\mathrm{C}_{6} \mathrm{H}_{5}, 2-\mathrm{Cl}-\mathrm{C}_{6} \mathrm{H}_{4}, 3-\mathrm{H}_{3} \mathrm{C}-\mathrm{C}_{6} \mathrm{H}_{4}, 3- \\
\mathrm{Cl}-\mathrm{C}_{6} \mathrm{H}_{4}, 3-\mathrm{O}_{2} \mathrm{~N}-\mathrm{C}_{6} \mathrm{H}_{4}, 4-\mathrm{Br}_{-}-\mathrm{C}_{6} \mathrm{H}_{4}, \\
4-\mathrm{H}_{3} \mathrm{CO}-\mathrm{C}_{6} \mathrm{H}_{4}\end{array}$ & $\mathrm{C}_{6} \mathrm{H}_{5}$ & MW & $81-94$ & 95 \\
\hline 3 & $\mathrm{C}_{6} \mathrm{H}_{5}$ & $\begin{array}{c}\text { 4- } \mathrm{H}_{3} \mathrm{C}-\mathrm{C}_{6} \mathrm{H}_{4}, 4-\mathrm{Cl}-\mathrm{C}_{6} \mathrm{H}_{4}, 4-\mathrm{H}_{3} \mathrm{CO}- \\
\mathrm{C}_{6} \mathrm{H}_{4}, t-\text { Butyl }\end{array}$ & MW & $77-97$ & 95 \\
\hline
\end{tabular}




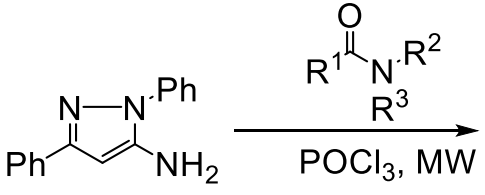

23

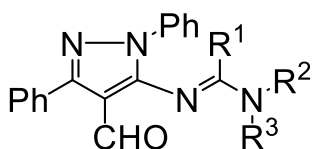

25

Scheme 11. Synthesis of $N$-N-Disubstituted-N'-[1,3-diphenyl-4-formyl-1H-pyrazol-5-yl]formimidamides 25.

Table 5. Yields (\%) of compounds 25

\begin{tabular}{|c|c|c|c|c|}
\hline & \multicolumn{3}{|c|}{ Amide solvents } & \multirow[t]{2}{*}{ Yield \% } \\
\hline & $\mathrm{R}^{1}$ & $\mathrm{R}^{2}$ & $\mathrm{R}^{3}$ & \\
\hline $3 a$ & $\mathrm{H}$ & $\mathrm{C}_{2} \mathrm{H}_{5}$ & $\mathrm{C}_{2} \mathrm{H}_{5}$ & 91 \\
\hline $3 b$ & $\mathrm{H}$ & \multicolumn{2}{|c|}{ Pyrrolidinyl } & 96 \\
\hline $3 c$ & $\mathrm{H}$ & \multicolumn{2}{|c|}{ Piperidinyl } & 92 \\
\hline
\end{tabular}

\subsection{Oxidation of the corresponding alcohols}

Somnath et al. ${ }^{96}$ reported that the oxidation of hydroxymethylpyrazole derivatives 26a-c or 27a-c in the presence of pyridinium chlorochromate (PCC) yielded the corresponding pyrazole-carboxaldehydes 28a-c and 29a-c, respectively, in 55-57\% and $75-80 \%$ yields (Scheme 12 ).<smiles>[R]c1ccc(-c2cc(CO)n(-c3ccccc3)n2)cc1</smiles>

27a-c

$R$
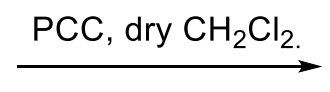

26-29: $\mathrm{a} ; \mathrm{R}=\mathrm{H}, \mathrm{b} ; \mathrm{R}=\mathrm{CH}_{3}, \mathrm{c} ; \mathrm{R}=\mathrm{Cl}$<smiles>[R]c1ccc(-c2cc(C=O)nn2-c2ccccc2)cc1</smiles>

28a-c<smiles>[R]c1ccc(-c2cc(C=O)[nH]n2)cc1</smiles>

29a-c

Scheme 12. Synthesis of pyrazole-carboxaldehydes 28a-c and 29a-c.

The oxidation of hydroxymethyl-(3-pyridyl)pyrazole derivative 30 with PCC afforded (3-pyridyl)pyrazole4-carboxaldehyde 31 in $41 \%$ yield (Scheme 13). ${ }^{97}$

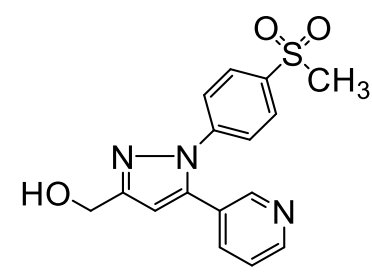

30

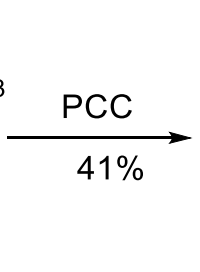

$\mathrm{O}$

31

Scheme 13. Synthesis of (3-pyridyl)pyrazole-4-carboxaldehyde 31. 
Ferrocene-based pyrazole-carboxaldehyde $\mathbf{3 3}$ has been formed by oxidation of hydroxymethylpyrazole linked to ferrocene 32 with manganese dioxide $\left(\mathrm{MnO}_{2}\right)$ in dichloromethane (Scheme 14). ${ }^{98}$

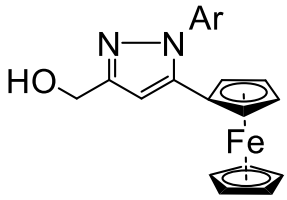

32

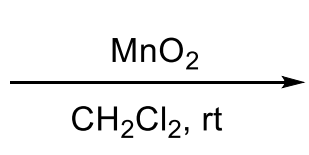

33

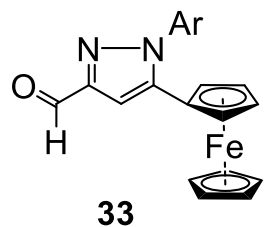

$82-94 \%$

$\mathrm{Ar}=\mathrm{C}_{6} \mathrm{H}_{5}$, Naphthalen-1-yl, 4- $\mathrm{H}_{3} \mathrm{C}-\mathrm{C}_{6} \mathrm{H}_{4}, 4-\mathrm{H}_{3} \mathrm{CO}-\mathrm{C}_{6} \mathrm{H}_{4}$, 4-tert-Butyl- $\mathrm{C}_{6} \mathrm{H}_{4}, 3-\mathrm{F}-\mathrm{C}_{6} \mathrm{H}_{4}, 4-\mathrm{F}-\mathrm{C}_{6} \mathrm{H}_{4}, 2-\mathrm{Cl}-\mathrm{C}_{6} \mathrm{H}_{4}, 3-\mathrm{Cl}-$ $\mathrm{C}_{6} \mathrm{H}_{4}, 3-\mathrm{Cl}-2-\mathrm{F}-\mathrm{C}_{6} \mathrm{H}_{3}, \mathrm{CH}_{3}$.

Scheme 14. Synthesis of ferrocene-based pyrazole-carboxaldehyde 33.

1,3-Diaryl-1H-pyrazole-4-carboxaldehydes 2 were prepared in good to excellent yields via the oxidation of the corresponding (1,3-diaryl-1H-pyrazol-4-yl)methanol 34 by iron(III) chloride hexahydrate $\mathrm{FeCl}_{3} .6 \mathrm{H}_{2} \mathrm{O}$ catalyzed by a free radical 2,2,6,6-tetramethylpiperidine-1-oxyl (TEMPO) (Scheme 15). ${ }^{99}$

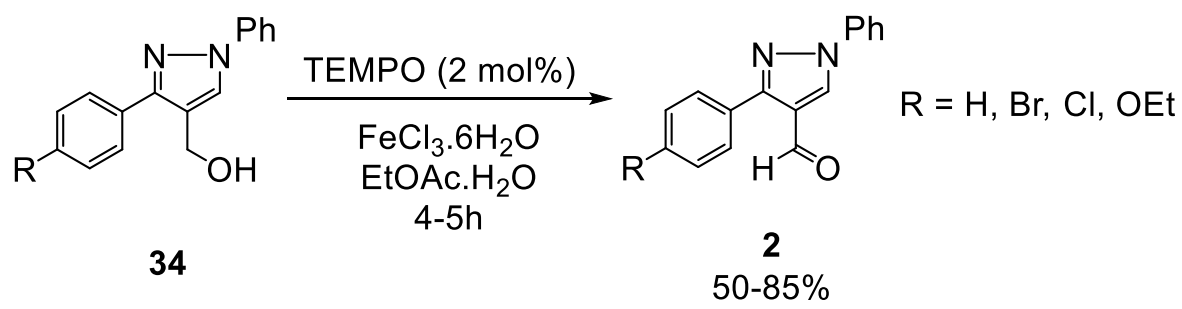

Scheme 15. Synthesis of 1,3-diaryl-1H-pyrazole-4-carboxaldehydes 2 from alcohol 34.

\subsection{Reduction of the corresponding pyrazolecarbonitrile}

The reduction of 5-amino-1-[2,6-dichloro-4-(trifluoromethyl)phenyl]-4-(methylsulfinyl)-1H-pyrazole-3carbonitrile 35 in the presence of di-iso-butylaluminium hydride (iso-Bu) ${ }_{2} \mathrm{AlH}$ afforded the corresponding pyrazole-4-carboxaldehyde 2 (Scheme 16). ${ }^{100}$

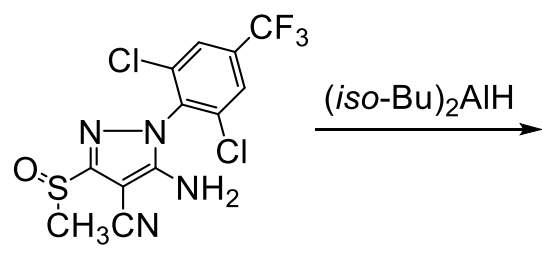

35<smiles>CC(O)c1nn(-c2c(Cl)cc(C(F)(F)F)cc2Cl)c(N)c1S(C)=O</smiles>

2

Scheme 16. Synthesis of pyrazole-4-carboxaldehyde 2 by reduction of pyrazolecarbonitrile 35 . 


\subsection{Hydrolysis of (pyrazolyl)methanimine}

Hydrolysis of $\mathrm{N}$-aryl-1-(3-(trimethylsilyl)-1H-pyrazol-4- yl)methanimine 36 gave 3-(trimethylsilyl)-1H-pyrazole4-carboxaldehyde 37 (Scheme 17). ${ }^{101}$

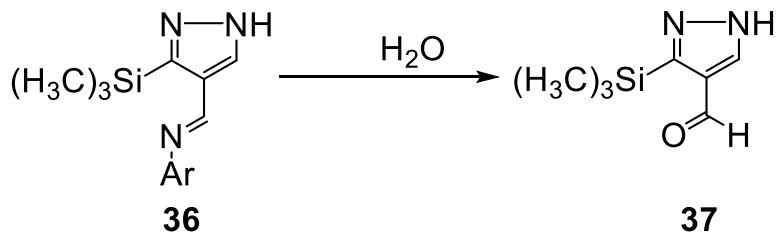

Scheme 17. Synthesis of 3-(trimethylsilyl)-1H-pyrazole-4-carboxaldehyde 37.

\subsection{Miscellaneous methods}

Arbačiauskienè et al. ${ }^{83}$ reported that the treatment of 3-(benzyloxy)-4-bromo-1-phenyl-1H-pyrazole 38 with $n$ BuLi gave rise to selective bromine-lithium exchange. Subsequent quenching of the intermediate 4lithiopyrazole with DMF afforded pyrazole-carboxaldehyde $\mathbf{2}$ (Scheme 18).<smiles>Brc1cn(-c2ccccc2)nc1OCc1ccccc1</smiles>

38

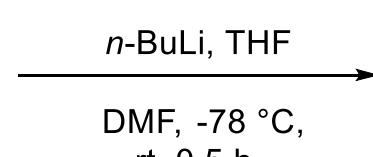

$\mathrm{rt}, 0.5 \mathrm{~h}$,<smiles>O=Cc1cn(-c2ccccc2)nc1OBr</smiles>

2 $70 \%$

Scheme 18. Synthesis of pyrazole-carboxaldehyde 2.

\section{Synthesis of Pyrazole-substituted Heterocycles}

\subsection{Pyrazole-substituted monoheterocyclic ring}

3.1.1. Monocyclic five-membered with one heteroatom. 3.1.1.1. Furan derivatives. Fekri et al. ${ }^{102}$ reported that the one-pot multi-component reaction of 4-pyrazole-carboxaldehyde 2 , ethyl pyruvate $\mathbf{3 9}$, and bromine gave 2,3-dihydrofuranediones 40 under ultrasonic irradiation (Scheme 19).

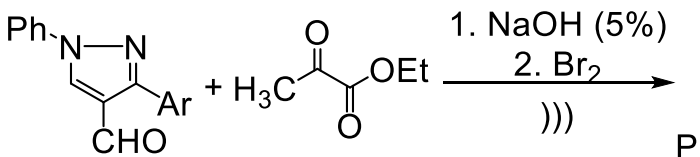

2<smiles>O=C1OC(c2cn[nH]c2)C(Br)C1=O</smiles><smiles>[R]c1ccc(C=[Te])c([R])c1[R]</smiles>

85-92\%

$$
\mathrm{R}^{1}=\mathrm{H}, \mathrm{Cl} ; \mathrm{R}^{2}=\mathrm{H}, \mathrm{NO}_{2} ; \mathrm{R}^{3}=\mathrm{OCH}_{3}, \mathrm{OH}, \mathrm{H}, \mathrm{Cl} \text {. }
$$

Scheme 19. Synthesis of 2,3-dihydrofuranediones 40 .

3.1.1.2. Pyrrole derivatives. Ragab et al. ${ }^{67}$ reported that the Claisene-Schmidt condensation between pyrazolecarboxaldehydes $\mathbf{2}$ and 4-chloroacetophenone $\mathbf{4 1}$ afforded the corresponding chalcones $\mathbf{4 2}$ which upon reaction with 4-substituted-benzaldehyde 43 in DMF in the presence of potassium cyanide as a catalyst afforded 1,4- 
diketones 44. Compounds $\mathbf{4 4}$ were cyclized using ammonium acetate in acetic acid under Paale-Knorr reaction conditions to yield the corresponding pyrrole derivatives 45 (Scheme 20).

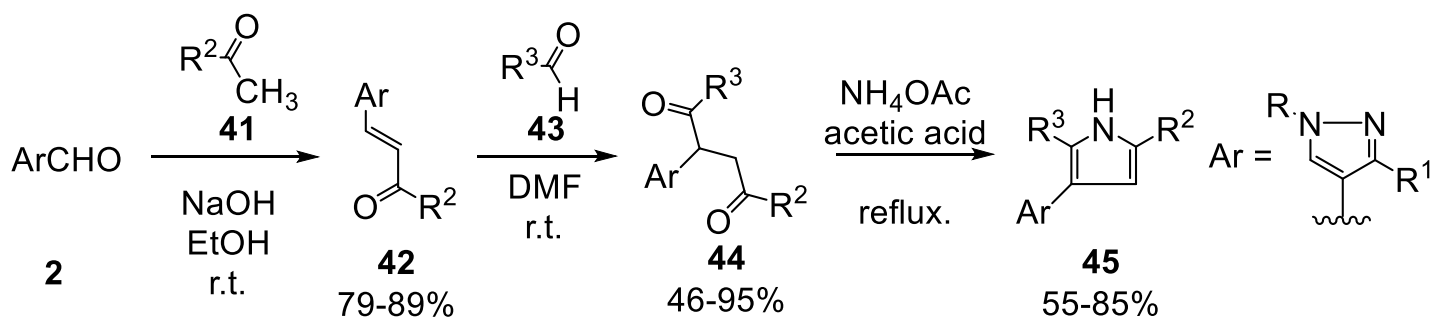

$\mathrm{R}=4-\mathrm{Cl}-\mathrm{C}_{6} \mathrm{H}_{4}, 4-\mathrm{H}_{2} \mathrm{NO}_{2} \mathrm{~S}-\mathrm{C}_{6} \mathrm{H}_{4} ; \mathrm{R}^{1}=4-\mathrm{H}_{3} \mathrm{C}-\mathrm{C}_{6} \mathrm{H}_{4}, 4-\mathrm{H}_{3} \mathrm{CO}-\mathrm{C}_{6} \mathrm{H}_{4} ; \mathrm{R}^{2}=4-\mathrm{Cl}_{-} \mathrm{C}_{6} \mathrm{H}_{4} ; \mathrm{R}^{3}=4-\mathrm{H}_{3} \mathrm{CO}-\mathrm{C}_{6} \mathrm{H}_{4}, 4-\mathrm{F}-\mathrm{C}_{6} \mathrm{H}_{4}$.

Scheme 20. Synthesis of pyrrole derivatives 45 .

3.1.2. Monocyclic five-membered with two heteroatoms. 3.1.2.1. Pyrazole derivatives. 3.1.2.1.1. Synthesis of pyrazole derivatives from chalcone carrying pyrazole. Under different reaction conditions, a series of chalcones 42 were synthesized through the Claisen-Schmidt condensation of pyrazole-4-carboxaldehyde $\mathbf{2}$ with aryl(hetero)methylene ketones $\mathbf{4 1 . 2 6 , 5 7 , 5 9 , 1 0 3 - 1 1 6}$ The chalcone derivatives $\mathbf{4 2}$ were then reacted with hydrazine derivatives 46 to give the corresponding 4,5-dihydro- $1 H$-pyrazole $47 .{ }^{26,57,113-116,59,103-107,111,112}$ On the other hand, the reaction of chalcones $\mathbf{4 2}$ with hydrazine hydrate in the presence of acid gave the acylated 4,5-dihydro1 -pyrazole 48 ${ }^{104,107-109,114}$ (Scheme 21).

On the other hand, the same reaction of chalcones $\mathbf{4 2}$ with hydrazine hydrate or phenylhydrazine in the presence of acetic acid gave the corresponding $1 \mathrm{H}$-pyrazoles 49 and $\mathbf{5 0}$ respectively. ${ }^{59}$ The reaction of chalcones $\mathbf{4 2}$ with iodine in dimethyl sulfoxide (DMSO) followed by reaction with hydrazine hydrate afforded pyrazoles $\mathbf{5 1} \mathbf{1}^{59,106}$ (Scheme 22).

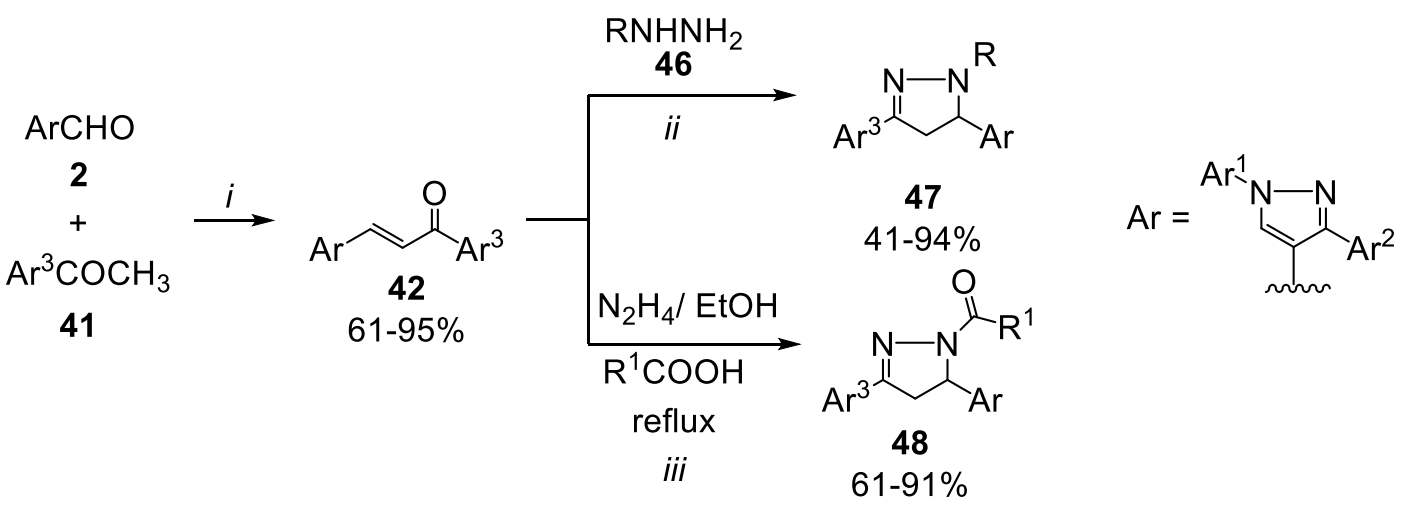

$\mathrm{Ar}^{1}=\mathrm{C}_{6} \mathrm{H}_{5}, 3-\mathrm{Cl}-\mathrm{C}_{6} \mathrm{H}_{4} ; \mathrm{Ar}^{2}=\mathrm{C}_{6} \mathrm{H}_{5}, 4-\mathrm{H}_{3} \mathrm{C}-\mathrm{C}_{6} \mathrm{H}_{4}, 4-\mathrm{O}_{2} \mathrm{~N}-\mathrm{C}_{6} \mathrm{H}_{4}, 4-\mathrm{F}-\mathrm{C}_{6} \mathrm{H}_{4}, 4-\mathrm{Cl}_{-} \mathrm{C}_{6} \mathrm{H}_{4}, 4-\mathrm{H}_{3} \mathrm{CO}-\mathrm{C}_{6} \mathrm{H}_{4}, 4-\mathrm{H}_{3} \mathrm{C}-\mathrm{C}_{6} \mathrm{H}_{4}, 4-\mathrm{Br}-$ $\mathrm{C}_{6} \mathrm{H}_{4}, 4-\mathrm{O}_{2} \mathrm{~N}-\mathrm{C}_{6} \mathrm{H}_{4}, 3-\mathrm{Br}_{-}-\mathrm{C}_{6} \mathrm{H}_{4}, 3-\mathrm{O}_{2} \mathrm{~N}-\mathrm{C}_{6} \mathrm{H}_{4}$, Pyridin-3-yl, Thiophen-2-yl, 5-Methylfuran-2-yl, Naphthalen-2-yl; Ar ${ }^{3}=$ $\mathrm{C}_{6} \mathrm{H}_{5}, 4-\mathrm{O}_{2} \mathrm{~N}-\mathrm{C}_{6} \mathrm{H}_{4}, 2-\mathrm{O}_{2} \mathrm{~N}-\mathrm{C}_{6} \mathrm{H}_{4}, 3-\mathrm{O}_{2} \mathrm{~N}-\mathrm{C}_{6} \mathrm{H}_{4}, 4-\mathrm{H}_{3} \mathrm{C}-\mathrm{C}_{6} \mathrm{H}_{4}, 2-\mathrm{H}_{3} \mathrm{C}-\mathrm{C}_{6} \mathrm{H}_{4}, 4-\mathrm{Cl}_{-} \mathrm{C}_{6} \mathrm{H}_{4}, 2-\mathrm{Cl}-\mathrm{C}_{6} \mathrm{H}_{4}, 3-\mathrm{Cl}_{-}-\mathrm{C}_{6} \mathrm{H}_{4}, 4-\mathrm{F}-\mathrm{C}_{6} \mathrm{H}_{4}, 2-$ $\mathrm{F}-\mathrm{C}_{6} \mathrm{H}_{4}, 3-\mathrm{F}-\mathrm{C}_{6} \mathrm{H}_{4}, 4-\mathrm{HO}-\mathrm{C}_{6} \mathrm{H}_{4}, 2-\mathrm{HO}-\mathrm{C}_{6} \mathrm{H}_{4}, 3-\mathrm{HO}-\mathrm{C}_{6} \mathrm{H}_{4}, 4-\mathrm{H}_{3} \mathrm{CO}-\mathrm{C}_{6} \mathrm{H}_{4}, 4-\mathrm{H}_{3} \mathrm{CO}-\mathrm{C}_{6} \mathrm{H}_{4}, 4-\mathrm{Br}-\mathrm{C}_{6} \mathrm{H}_{4}, 4-\mathrm{H}_{5} \mathrm{C}_{2}-\mathrm{C}_{6} \mathrm{H}_{4}, 5-\mathrm{H}_{3} \mathrm{C}-$ 2- $-\mathrm{HO}-\mathrm{C}_{6} \mathrm{H}_{3}, 5-\mathrm{Cl}-2-\mathrm{HO}-\mathrm{C}_{6} \mathrm{H}_{3}, 4-\mathrm{H}_{3} \mathrm{C}-2-\mathrm{HO}-\mathrm{C}_{6} \mathrm{H}_{3}, 3-\mathrm{H}_{3} \mathrm{C}-2-\mathrm{HO}_{-}-\mathrm{C}_{6} \mathrm{H}_{3}, 5-\mathrm{H}_{5} \mathrm{C}_{2}-2-\mathrm{HO}-\mathrm{C}_{6} \mathrm{H}_{3}, 5-\mathrm{Br}-2-\mathrm{HO}-\mathrm{C}_{6} \mathrm{H}_{3}, 5-\mathrm{F}-2-\mathrm{HO}-$ $\mathrm{C}_{6} \mathrm{H}_{3}, 3,5-\mathrm{di}-\mathrm{H}_{3} \mathrm{C}-2-\mathrm{HO}-\mathrm{C}_{6} \mathrm{H}_{2}, 3,5-\mathrm{di}-\mathrm{Cl}-2-\mathrm{HO}-\mathrm{C}_{6} \mathrm{H}_{2}, 4,6-\mathrm{di}-\mathrm{H}_{3} \mathrm{C}-2-\mathrm{HO}-\mathrm{C}_{6} \mathrm{H}_{2}, 5-\mathrm{Cl}-3-\mathrm{H}_{3} \mathrm{C}-2-\mathrm{HO}-\mathrm{C}_{6} \mathrm{H}_{2}, 2,4-\mathrm{di}-\mathrm{Cl}-\mathrm{C}_{6} \mathrm{H}_{3}$, 2,3-di- $\mathrm{H}_{3} \mathrm{CO}-\mathrm{C}_{6} \mathrm{H}_{3}$, Pyridin-3-yl, 10H-Phenothiazin-2-yl, Thiophen-2-yl, Benzofuran-2-yl, Furan-2-yl; $\mathrm{R}=\mathrm{H}, \mathrm{C}_{6} \mathrm{H}_{5}$, 4-Sulfamoylphenyl, $\mathrm{CSNH}_{2}$, 4-Phenylthiazol-2-yl, 4-Phenyl-5-(phenyldiazenyl)thiazol-2-yl; $\mathrm{R}^{1}=\mathrm{H}_{1} \mathrm{CH}_{3}, \mathrm{C}_{2} \mathrm{H}_{5}, \mathrm{C}_{3} \mathrm{H}_{7}$; i) $\mathrm{EtOH} / \mathrm{KOH} /$ reflux $^{106,111,112}$ or grindig, ${ }^{112} \mathrm{MeOH} / \mathrm{NaOH} /$ reflux, ${ }^{113} \mathrm{MeOH} / \mathrm{NaOH}$ r.t. , $57,110 \mathrm{EtOH} /$ 
$\mathrm{NaOH} ;{ }^{59,104,114,115}$ ii) two drops $\mathrm{AcOH} /$ stirring r.t. , ${ }^{111,116} \mathrm{MeOH} / \mathrm{HCl} / \mathrm{reflux},{ }^{113} \mathrm{AcOH} / \mathrm{reflux},{ }^{103} \mathrm{H}_{2} \mathrm{SO}_{4} / \mathrm{AcOH}$ reflux $^{26,112}$ or grindig, ${ }^{112} \mathrm{EtOH} / \mathrm{NaOH} /$ reflux $^{57,59,104,114} \mathrm{EtOH}^{59,104,112}$ grindig $^{112}$ or dioxan. ${ }^{105}$

Scheme 21. Synthesis of 4,5-dihydro-1H-pyrazole 47 and 4,5-dihydro-1H-pyrazole 48.

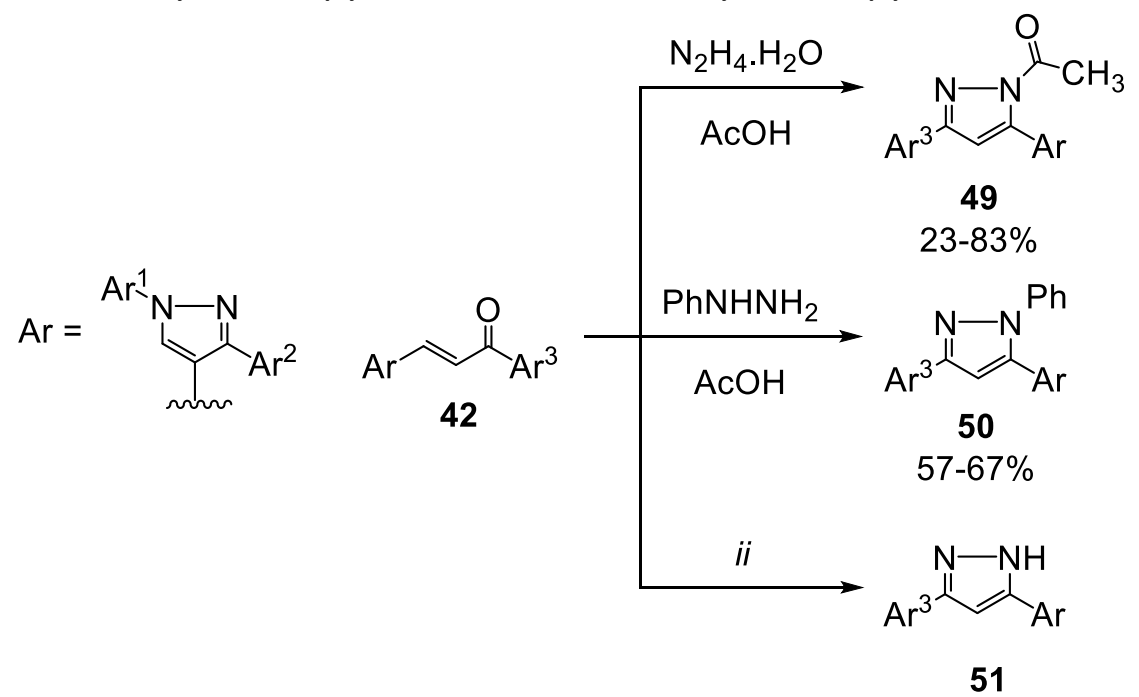

$\mathrm{Ar}^{1}=\mathrm{C}_{6} \mathrm{H}_{5} ; \mathrm{Ar}^{2}=\mathrm{C}_{6} \mathrm{H}_{5}$, Naphthalen-2-yl; $\mathrm{Ar}^{3}=5-\mathrm{H}_{3} \mathrm{C}-2-\mathrm{HO}_{-}-\mathrm{C}_{6} \mathrm{H}_{3}, 5-\mathrm{Cl}-2-\mathrm{HO}-\mathrm{C}_{6} \mathrm{H}_{3}, 4-\mathrm{H}_{3} \mathrm{C}-2-\mathrm{HO}-\mathrm{C}_{6} \mathrm{H}_{3}, 3-\mathrm{H}_{3} \mathrm{C}-2-\mathrm{HO}-$ $\mathrm{C}_{6} \mathrm{H}_{3}, 5-\mathrm{H}_{5} \mathrm{C}_{2}-2-\mathrm{HO}-\mathrm{C}_{6} \mathrm{H}_{3}, 5-\mathrm{Br}-2-\mathrm{HO}-\mathrm{C}_{6} \mathrm{H}_{3}, 5-\mathrm{F}-2-\mathrm{HO}_{-} \mathrm{C}_{6} \mathrm{H}_{3}, 3,5-\mathrm{di}-\mathrm{H}_{3} \mathrm{C}-2-\mathrm{HO}-\mathrm{C}_{6} \mathrm{H}_{2}, 3,5-\mathrm{di}-\mathrm{Cl}-2-\mathrm{HO}-\mathrm{C}_{6} \mathrm{H}_{2}, 4,6-\mathrm{di}-\mathrm{H}_{3} \mathrm{C}-$ 2- $-\mathrm{HO}_{6} \mathrm{C}_{6} \mathrm{H}_{2}, 5-\mathrm{Cl}-3-\mathrm{H}_{3} \mathrm{C}-2-\mathrm{HO}-\mathrm{C}_{6} \mathrm{H}_{2}, 10 \mathrm{H}$-Phenothiazin-2-yl; i) EtOH/ KOH/ reflux, $\left.{ }^{106} \mathrm{EtOH} / \mathrm{NaOH} ;{ }^{59} \mathrm{ii}\right)(1) \mathrm{I}_{2} / \mathrm{DMSO}$ 2) $\left.\mathrm{N}_{2} \mathrm{H}_{4} / \mathrm{EtOH}\right)^{106}$ or $\mathrm{N}_{2} \mathrm{H}_{4} / \mathrm{EtOH}^{59}$

Scheme 22. Synthesis of $1 H$-pyrazoles 49, 50 and 51.

Ali ${ }^{117}$ reported the synthesis of 1 -(dimethylphosphoryl)-3-(1,3-diphenyl-1H-pyrazol-4-yl)-5-phenyl- $1 H$ pyrazole 52 by reaction of 3-(1,3-diphenyl-1H-pyrazol-4-yl)-1-phenylprop-2-en-1-one 42 with $p, p$ dimethylphosphinic hydrazide $\mathbf{4 6}$ in the presence of acetic acid (Scheme 23).

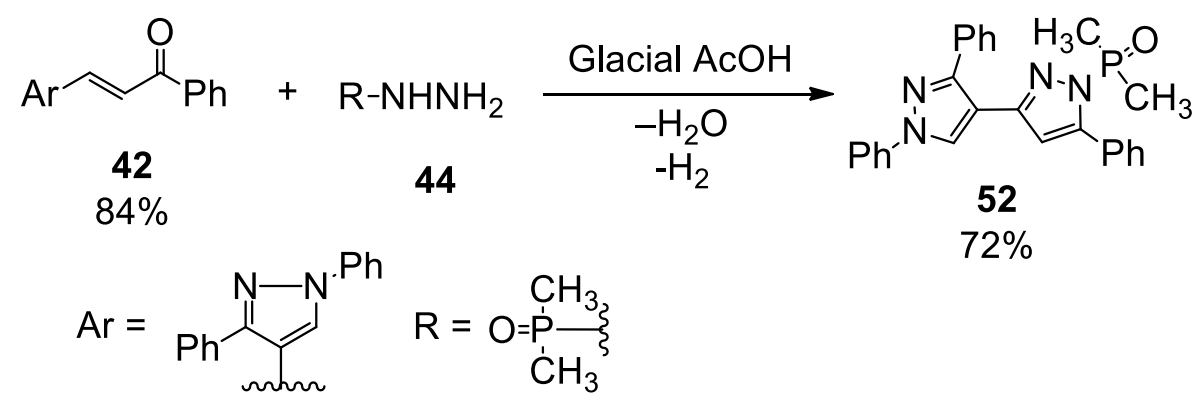

Scheme 23. Synthesis of 3-(1,3-diphenyl-1H-pyrazol-4-yl)-5-phenyl-1H-pyrazole 52.

3.1.2.1.2. Synthesis of pyrazole derivatives from arylidene malononitrile carrying pyrazole. Ismail et al. ${ }^{118}$ reported the synthesis of 2-((1-(3-chlorophenyl)-3-(4-methoxyphenyl)-1H-pyrazol-4yl)methylene)malononitrile 54 by heating of pyrazole-4-carboxaldehyde 2 and malononitrile 53 . Heating of 54 at reflux with hydrazine hydrate 46 in ethanol/ piperidine gave 4-((1-(3-chlorophenyl)-3-(4-methoxyphenyl)- $1 \mathrm{H}$ pyrazol-4-yl)methylene)-4H-pyrazole-3,5-diamine 55 (Scheme 24). 


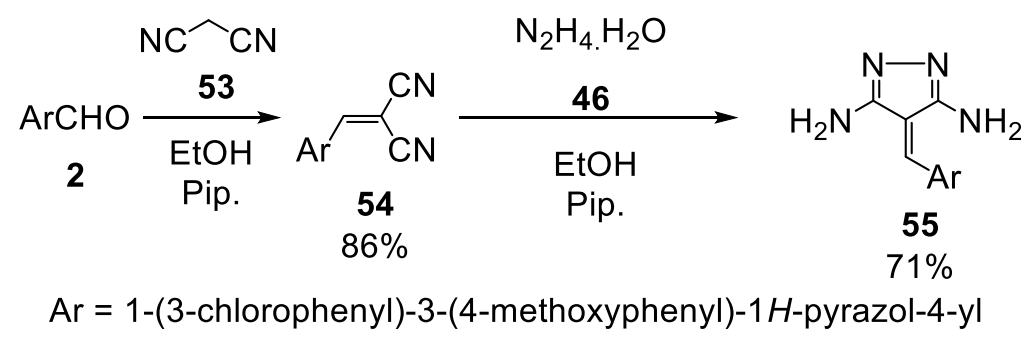

Scheme 24. Synthesis of 4-((1-(3-chlorophenyl)-3-(4-methoxyphenyl)-1H-pyrazol-4-yl)methylene)-4H-pyrazole3,5-diamine 55 .

On the other hand, the reaction of 2-((1,3-diphenyl-1H-pyrazol-4-yl)methylene)malononitrile 54 with p,p-dimethylphosphinic hydrazide afforded 5-amino-1-(dimethylphosphoryl)-3-(1,3-diphenyl-1H-pyrazol-4-yl)$1 H$-pyrazole-4-carbonitrile $\mathbf{5 6}^{117}$ (Scheme 25).

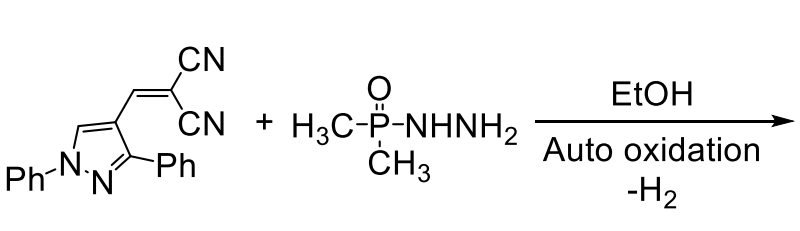

54

46<smiles></smiles>

56

$69 \%$

Scheme 25. Synthesis of 3-(1,3-diphenyl-1H-pyrazol-4-yl)-1H-pyrazole-4-carbonitrile 56.

3.1.2.1.3. Synthesis of pyrazole derivatives from cyanoacetohydrazide carrying pyrazole. Atta-Allah et al. ${ }^{119}$ reported that treatment of the pyrazole-4-carboxaldehyde $\mathbf{2}$ with cyanoacetohydrazide $\mathbf{5 7}$ in dioxane at reflux gave 2-cyano- $N^{\prime}$-[(1,3-diphenyl-1H-pyrazol-4-yl)methylene]acetohydrazide 58 . Heating $N$-condensation product 58 in ethanol in the presence of a catalytic amount of piperidine, gave a mixture of pyrazolone and hydroxyl pyrazole derivatives 59 in a ratio of 2:3 (Scheme 26).

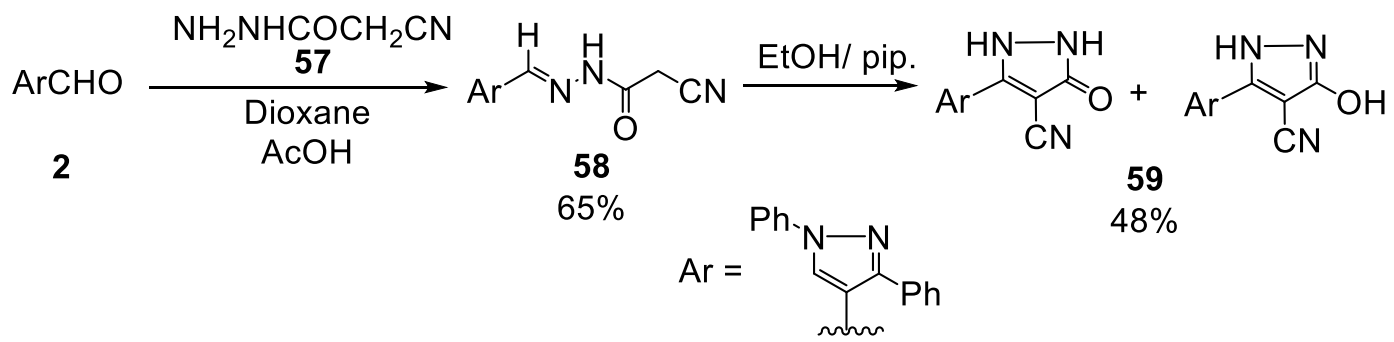

Scheme 26. Synthesis of a mixture of pyrazolone and hydroxyl pyrazole derivatives 59.

3.1.2.1.4. Synthesis of pyrazole derivatives from cyanoacrylohydrazide carrying pyrazole. Fahmy et al..$^{120}$ reported that heating of 1-(3-chlorophenyl)-3-(4-methoxyphenyl)-1H-pyrazole-4-carboxaldehyde 2 with cyanoacetohydrazide $\mathbf{5 7}$ in ethanol containing a few drops of acetic acid gave pyrazolinone derivative $\mathbf{6 0}$. Stirring of pyrazole-carboxaldehyde $\mathbf{2}$ and cyanoacetohydrazide $\mathbf{5 7}$ in ethanol containing few drops of triethylamine 
gave 3-[1-(3-chlorophenyl)-3-(4-methoxyphenyl)-1H-pyrazol-4-yl]-2-cyanoacrylohydrazide 61. Heating of 61 at reflux in acetic acid afforded compounds 60 (Scheme 27).

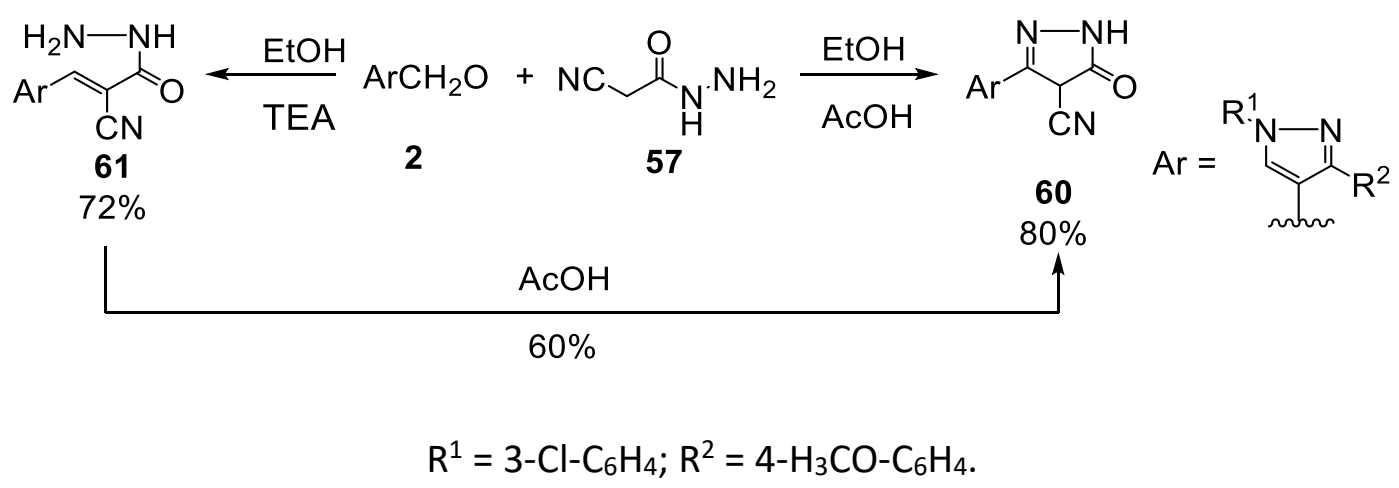

Scheme 27. Synthesis of pyrazolinone derivatives 60 .

3.1.2.1.5. Miscellaneous methods. Youssef et al. ${ }^{121}$ reported that heating of quinazolinone derivative 61 with 1,3-diphenyl-1H-pyrazole-4-carboxaldehyde 2 in ethanol at reflux followed by heating with hydrazine hydrate at reflux gave 5-(1,3-diphenyl-1H-pyrazol-4-yl)-3H-pyrazol-3-one 63 and 3-amino-2-methylquinazolin-4(3H)one 62 as a by-product (Scheme 28).

Muthineni et al. ${ }^{122}$ reported that the four-component reaction of hydrazine hydrate 46 , ethylacetate derivative 64, pyrazole-carboxaldehyde 2, and 3,5-dimethyl isoxazole 65 afforded the corresponding 5-methyl4-(2-(3-methyl-4-nitroisoxazol-5yl)-1-arylethyl)-1H-pyrazol-3-oles 66 (Scheme 29).

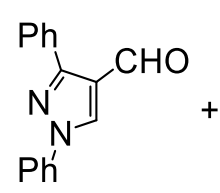

2<smiles>Cc1nc2ccccc2c(=O)n1NC(=O)CC#N</smiles>

61
1) $10 \% \mathrm{KOH}$ EtOH, Reflux
2) $\mathrm{N}_{2} \mathrm{H}_{4} \cdot \mathrm{H}_{2} \mathrm{O}$ n-butanol<smiles>Cc1nc2ccccc2c(=O)n1N</smiles>

62<smiles>O=C1C=C(c2cn(-c3ccccc3)nc2-c2ccccc2)N=N1</smiles>

63 $90 \%$

Scheme 28. Synthesis of 3-amino-2-methylquinazolin-4(3H)-one 62 and 5-(1,3-diphenyl-1H-pyrazol-4-yl)-3Hpyrazol-3-one 63.

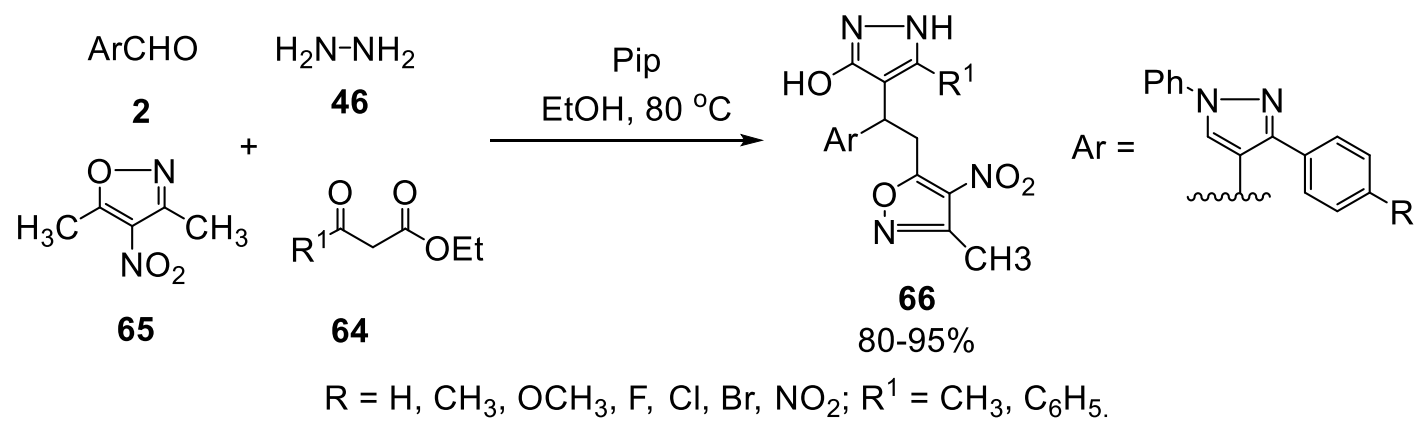

Scheme 29. Synthesis of 5-methyl-4-(2-(3-methyl-4-nitroisoxazol-5yl)-1-arylethyl)-1H-pyrazol-3-oles 66. 
3.1.2.2. Imidazole derivatives. Abou Elmagd et al. ${ }^{123}$ reported that heating of $N$-(1-(1,3-diphenyl-1H-pyrazol-4yl)-3-oxo-3-(2-((phenyl-I2-azaneyl)carbonothioyl) hydrazineyl) prop-1-en-2-yl)benzamide $67^{124}$ with 0 phenylenediamine 68 in ethanol at reflux produced a mixture of 3-amino-5-((1,3-diphenyl-1H-pyrazol-4$\mathrm{yl}$ )methylene)-2-phenyl-3,5-dihydro-4H-imidazol-4-one 69 and 1,3-dihydro-2H-benzo[d]imidazole-2-thione 70 (Scheme 30).<smiles>CCN(c1ccccc1)c1ccccc1N</smiles>

Scheme 30. Synthesis of 3,5-dihydro-4H-imidazol-4-one 69 and $2 H$-benzo[d]imidazole-2-thione 70.

Aly et al. $^{125}$ reported that heating of 3-aryl-1-phenyl-1H-pyrazole-4-carboxaldehydes 2 with benzamidine hydrochloride 71 and ethyl chloroacetate 64 gave dihydroimidazolone derivatives 72 (Scheme 31).

Heating of pyrazole-4-carboxaldehyde $\mathbf{2}$ with the appropriate isothiocyanate $\mathbf{7 3}$ and glycine $\mathbf{7 4}$ in glacial acetic acid at reflux afforded thioxoimidazolidin-4-ones $\mathbf{7 5}^{118,126}$ (Scheme 32).

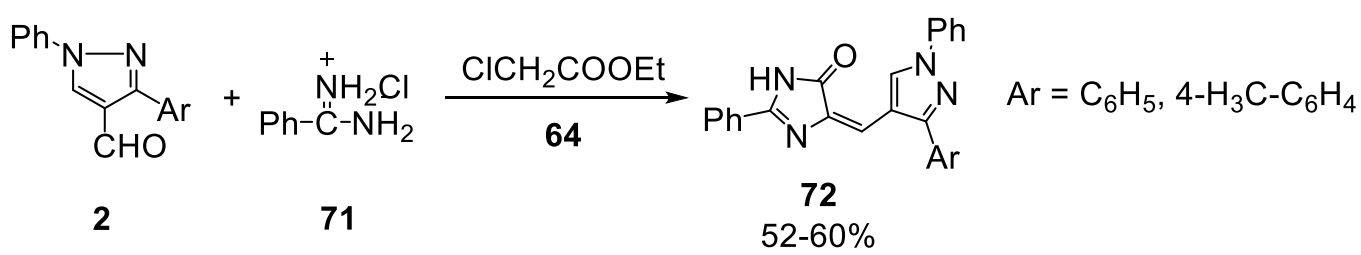

Scheme 31. Synthesis of dihydroimidazolone derivatives $\mathbf{7 2 .}$

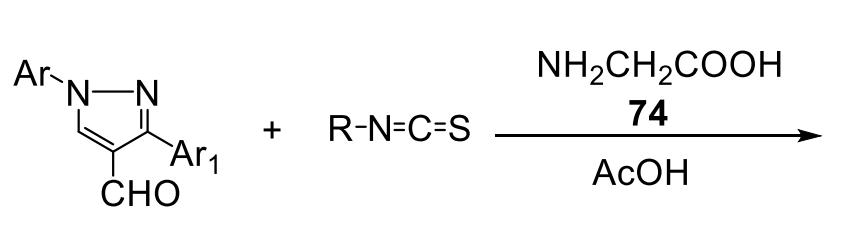

2
73

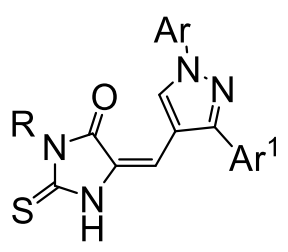

75

$\mathrm{Ar}=\mathrm{C}_{6} \mathrm{H}_{5}, 3-\mathrm{Cl}-\mathrm{C}_{6} \mathrm{H}_{4} ; \mathrm{Ar}^{1}=4-\mathrm{H}_{3} \mathrm{CO}-\mathrm{C}_{6} \mathrm{H}_{4}, \mathrm{H}_{5} \mathrm{C}_{6}-\mathrm{CH}_{2} \mathrm{O}-\mathrm{C}_{6} \mathrm{H}_{5} ; \mathrm{R}=\mathrm{C}_{6} \mathrm{H}_{5}, 3-\mathrm{Cl}-\mathrm{C}_{6} \mathrm{H}_{4}, 4-\mathrm{H}_{3} \mathrm{CO}-\mathrm{C}_{6} \mathrm{H}_{4}$.

Scheme 32. Synthesis of thioxoimidazolidin-4-ones 75.

Shirole et al. ${ }^{127}$ reported that imidazoles 79 were synthesized by a multi-component reaction of benzil 76, aniline derivatives 77, 1-phenyl-3- $p$-tolyl-1H-pyrazole-4-carboxaldehyde 2 and ammonium acetate 78 in the presence of 1-butyl-3-methyl-1-imidazolium tetrafluoroborate [BMIM] $\left[\mathrm{BF}_{4}\right]$ as a catalyst (Scheme 33). 


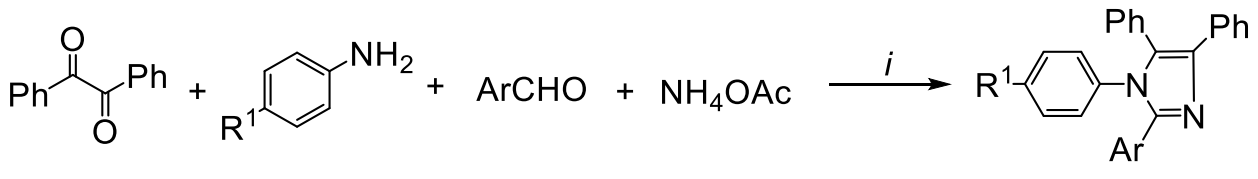

76 77

2

78

79

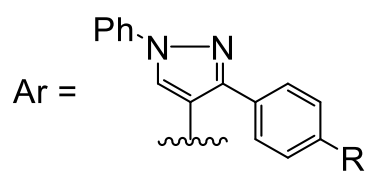

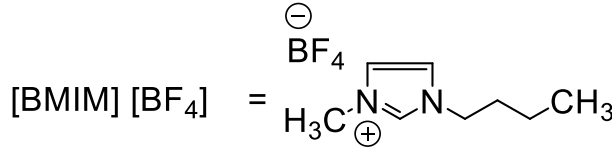

$\mathrm{R}=\mathrm{CH}_{3}, \mathrm{H}, \mathrm{F}, \mathrm{Cl}, \mathrm{Br}, \mathrm{NO}_{2} ; \mathrm{R}^{1}=\mathrm{H}, \mathrm{Cl} ; \boldsymbol{i}=$ Conventional Method: Reflux 14-15 h / [BMIM] [BF $] /$ Ethanol (68- 78\%); Green Method: MW at 240 W/ [BMIM][BF4]/Solvent Free 10-12 min (84- 89\%).

Scheme 33. Synthesis of imidazoles 79.

Banothu et al. ${ }^{128}$ reported that 1,3-diphenyl-4-(4,5-diphenyl-1H-imidazol-2-yl)- $1 \mathrm{H}$-pyrazoles 81 were synthesized by the condensation of benzoin $\mathbf{8 0}$ with 1,3-diphenyl-1H-pyrazole-4-carboxaldehyde $\mathbf{2}$ and ammonium acetate 78 using Brønsted acidic ionic liquid, (4-sulfobutyl)tris(4-sulfophenyl) phosphonium hydrogen sulfate [(4-SB)T(4-SPh)PHSO 4$]$ as a catalyst (Scheme 34).

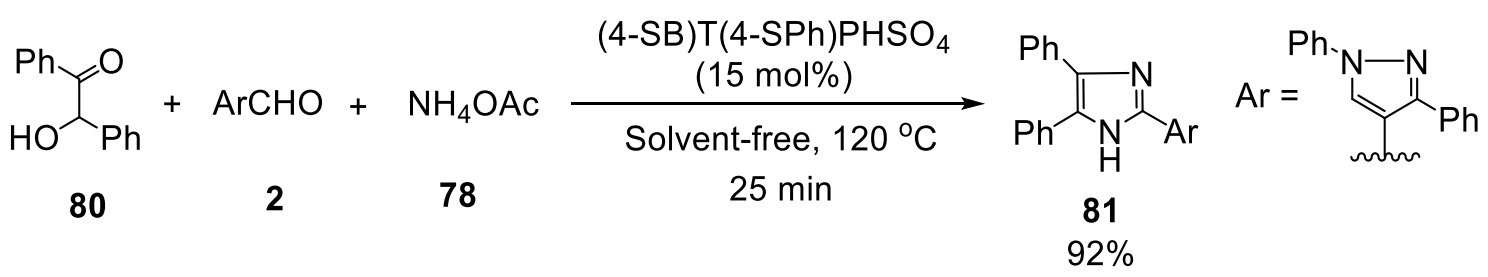

Scheme 34. Synthesis of 1,3-diphenyl-4-(4,5-diphenyl-1H-imidazol-2-yl)-1H-pyrazoles 81.

Under different reaction conditions, a series of imidazolylpyrazoles $\mathbf{8 2}$ was synthesized by the multicomponent reaction of pyrazole-4-carboxaldehydes 2 , benzil 76 , and ammonium acetate $\mathbf{7 8}^{128-132}$ (Scheme 35).

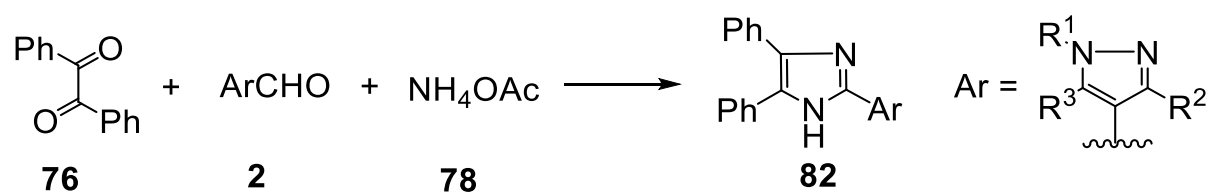

Reaction conditions; a: $\mathrm{NH}_{4} \mathrm{OAc} /$ Acetic acid / Reflux (69-95\%), ${ }^{129-131}$ b: $\mathrm{NH}_{4} \mathrm{OAc} /$ Acetic acid / MW (84-91\%), ${ }^{129}$ c: $\mathrm{NH}_{4} \mathrm{OAc} /$ Glutamic acid / ethanol / Reflux (85-94\%), ${ }^{129} \mathrm{~d}: \mathrm{NH}_{4} \mathrm{OAc} / 120^{\circ} \mathrm{C}$ using Brønsted acidic ionic liquid, [(4-SB)T(4-SPh)PHSO 4 ] (98\%), ${ }^{128}$ e: $\mathrm{NH}_{4} \mathrm{OAc} /$ Reflux 4- 4.5hrs/ [BMIM] [BF $]$ / Ethanol (68-72\%), ${ }^{132} \mathbf{f : ~} \mathrm{NH}_{4} \mathrm{OAc} /$ Ultra-sonication (80-90 min)[BMIM] [BF $] /$ Ethanol (78-80\%), ${ }^{132}$ g: $\mathrm{NH}_{4} \mathrm{OAc} / \mathrm{MW}$ irradiation 240 watt (7-9 min) [BMIM] [BF4]/ Solvent Free (80-86\%); ${ }^{132} \mathrm{R}^{1}=\mathrm{C}_{6} \mathrm{H}_{5}, 4-\mathrm{O}_{2} \mathrm{~N}-\mathrm{C}_{6} \mathrm{H}_{4} ; \mathrm{R}^{2}=\mathrm{C}_{6} \mathrm{H}_{5}, 4-\mathrm{Cl}-\mathrm{C}_{6} \mathrm{H}_{4}, 4-\mathrm{F}-\mathrm{C}_{6} \mathrm{H}_{4}, 4-\mathrm{Br}-\mathrm{C}_{6} \mathrm{H}_{4}, 4-\mathrm{O}_{2} \mathrm{~N}-$ $\mathrm{C}_{6} \mathrm{H}_{4}, 4-\mathrm{H}_{3} \mathrm{CO}-\mathrm{C}_{6} \mathrm{H}_{4}, 4-\mathrm{H}_{3} \mathrm{C}-\mathrm{C}_{6} \mathrm{H}_{4}, 3-\mathrm{O}_{2} \mathrm{~N}-\mathrm{C}_{6} \mathrm{H}_{4}, 4-\left(\mathrm{H}_{5} \mathrm{C}_{6}\right)-\mathrm{C}_{6} \mathrm{H}_{4}, 3,4-d i C l-\mathrm{C}_{6} \mathrm{H}_{3}, 3,4-d i F-\mathrm{C}_{6} \mathrm{H}_{3}$, 2-Thienyl, 2-Fluorobenzyl, $\mathrm{CH}_{3}$, Coumarinyl, 6-Br-coumarinyl; $\mathrm{R}^{3}=\mathrm{H}, \mathrm{CH}_{3}$.

Scheme 35. Synthesis of imidazolylpyrazoles $\mathbf{8 2}$. 
3.1.2.3. Oxazole derivatives. Bekhit and Fahmy ${ }^{133}$ reported the synthesis of oxazolidine-3-carboxylate 84 via reaction of 3-(5-bromo-2-thienyl)-1-phenyl-1H-pyrazole-4-carboxaldehyde 2 with L-serine 83, followed by $N$ protection using di-tert-butyl dicarbonate $(\mathrm{Boc})_{2} \mathrm{O}$ (Scheme 36$)$.<smiles>O=Cc1cn(-c2ccccc2)nc1-c1ccc(Br)s1</smiles>

2<smiles>NC(O)C(=O)O</smiles>

83 83 $\underset{\text { ii) }(\mathrm{Boc})_{2} \mathrm{O}}{\stackrel{\text { Stirring }}{\longrightarrow}}$<smiles>CC(C)(C)OC(=O)N1C(C(=O)O)COC1c1cnn(P)c1</smiles>

Scheme 36. Synthesis of oxazolidine-3-carboxylate 84.

Aly et al. ${ }^{125}$ reported that heating of 3-aryl-1-phenyl-1H-pyrazole-4-carboxaldehydes 2 with glycine derivatives 74 and sodium acetate in acetic anhydride afforded the corresponding 4-(3-aryl-1-phenyl- $1 H$ pyrazole-4-ylmethylene)-2-substituted oxazol-5-(4H)-ones 85 (Scheme 37).

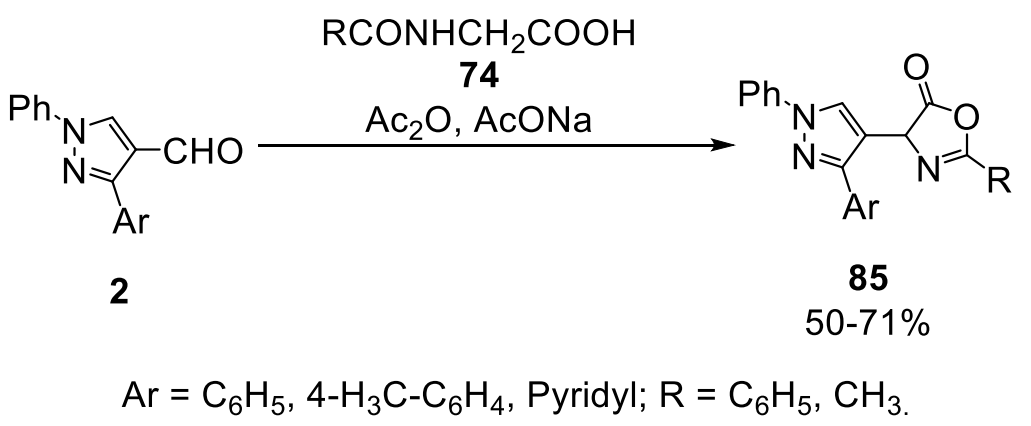

Scheme 37. Synthesis of 2-substituted oxazol-5-(4H)-ones 85.

3.1.2.4. Isoxazole derivatives. Madhavilatha et $a l .{ }^{134}$ reported that pyrazole-carboxaldehydes 2 was converted to 4-hydroxypyrazoles 86 upon treatment with meta-chloroperoxybenzoic acid (mCPBA). Next, 4hydroxypyrazoles $\mathbf{8 6}$ reacted with propargyl bromide $\mathbf{8 7}$ in tetrahydrofuran (THF)/ DMF using NaH as a base to give $\mathbf{O}$-propargylated pyrazole derivative $\mathbf{8 8}$. The reaction of $\mathbf{8 8}$ with aryl aldoximes $\mathbf{8 9}$ afforded isoxazole functionalized pyrazole derivatives 90 (Scheme 38).

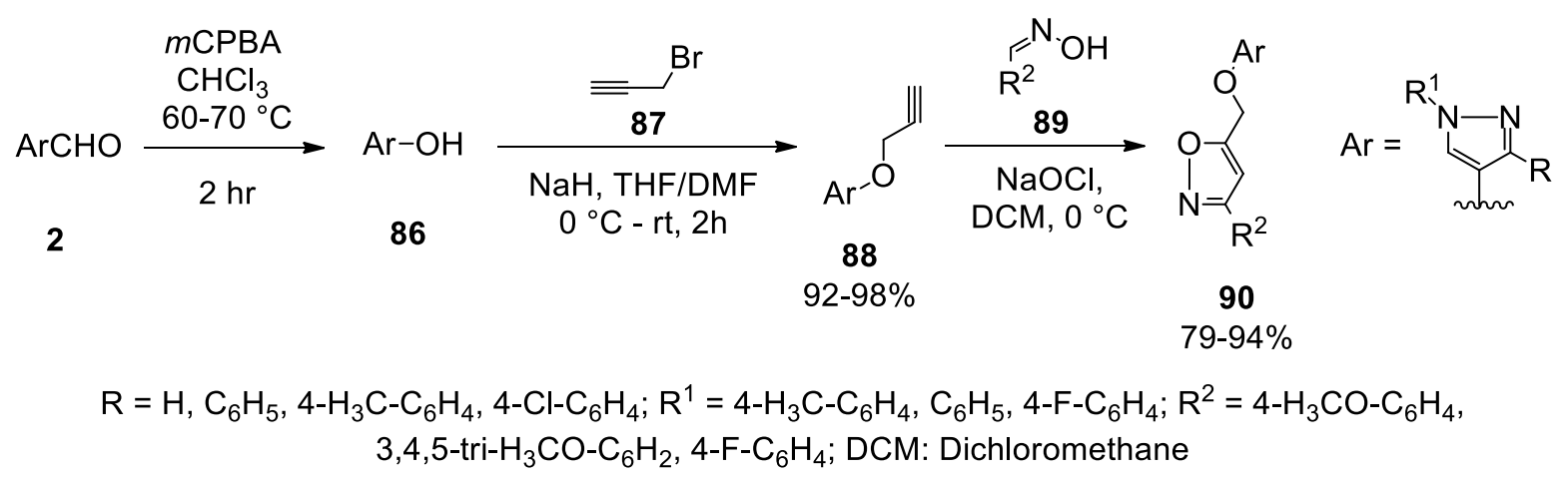

Scheme 38. Synthesis of isoxazole functionalized pyrazole derivatives $\mathbf{9 0 .}$ 
Reaction of $\alpha, 6$-unsaturated ketones $\mathbf{4 2}$ with an aqueous solution of hydroxylamine hydrochloride $\mathbf{9 1}$, and

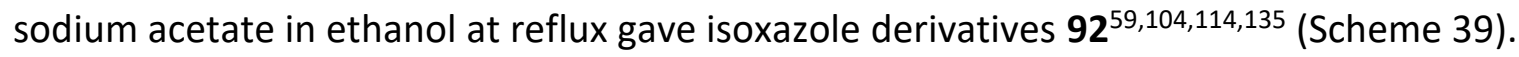

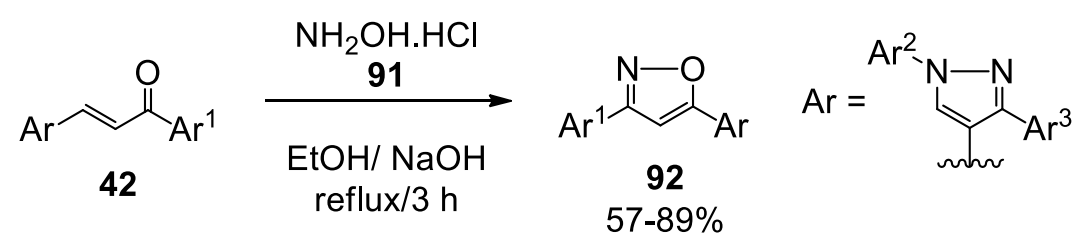

$\mathrm{Ar}^{1}=\mathrm{C}_{6} \mathrm{H}_{5}, 4-\mathrm{H}_{2} \mathrm{~N}-\mathrm{C}_{6} \mathrm{H}_{4}, 4-\mathrm{Cl}-\mathrm{C}_{6} \mathrm{H}_{4}, 4-\mathrm{Br}-\mathrm{C}_{6} \mathrm{H}_{4}, 4-\mathrm{F}-\mathrm{C}_{6} \mathrm{H}_{4}, 2-\mathrm{HO}_{6}-\mathrm{C}_{6} \mathrm{H}_{4}, 4-\mathrm{HO}-\mathrm{C}_{6} \mathrm{H}_{4}, 4-\mathrm{H}_{3} \mathrm{CO}-\mathrm{C}_{6} \mathrm{H}_{4}, 4-\mathrm{H}_{3} \mathrm{C}-\mathrm{C}_{6} \mathrm{H}_{4}, 3-\mathrm{O}_{2} \mathrm{~N}-$

$\mathrm{C}_{6} \mathrm{H}_{4}, 4-\mathrm{O}_{2} \mathrm{~N}-\mathrm{C}_{6} \mathrm{H}_{4}$, Thiophen-2-yl, 10H-Phenothiazin-2-yl; $\mathrm{Ar}^{2}=\mathrm{C}_{6} \mathrm{H}_{5}, 3-\mathrm{Cl}_{-} \mathrm{C}_{6} \mathrm{H}_{4} ; \mathrm{Ar}^{3}=\mathrm{C}_{6} \mathrm{H}_{5}, 2-\mathrm{H}_{3} \mathrm{CO}-\mathrm{C}_{6} \mathrm{H}_{4}, 4-$

$$
\mathrm{H}_{3} \mathrm{CO}-\mathrm{C}_{6} \mathrm{H}_{4}
$$

Scheme 39. Synthesis of isoxazole derivatives 92.

3.1.2.5. Thiazole derivatives. Heating a solution of pyrazole-carboxaldehyde 2 with $L$-cysteine $\left(R^{3}=H\right)$ or $L$ penicillamine $\left(\mathrm{R}^{3}=\mathrm{CH}_{3}\right) \mathbf{8 3}$ followed by $\mathrm{N}$-protection using $(\mathrm{Boc})_{2} \mathrm{O}$ provided thiazolidine-4-carboxylic acid 93. ${ }^{133,136}$ Condensation of pyrazole-4-carboxaldehyde $\mathbf{2}$ with arylamine $\mathbf{7 7}$ gave Schiff's bases $\mathbf{9 4}$ which reacted

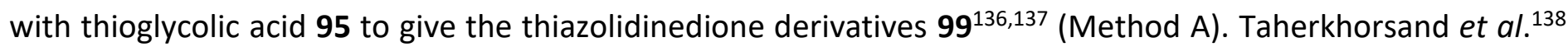
and Nikpassand et al. ${ }^{139}$ reported also the synthesis of 2-pyrazole-3-phenyl-1,3-thiazolidine-4-ones 96 via a multi-component reaction of pyrazole-carboxaldehydes 2, arylamine 77, thioglycolic acid 95 in the presence of DSDABCOC $^{138}$ or OImDSA ${ }^{139}$ (Method B) (Scheme 40).

DSDABCOC: ionic liquid 1,4-disulfo-1,4-diazoniabicyclo[2.2.2]octane chloride

OImDSA: 2-oxoimidazolidine-1,3-disulfonic acid

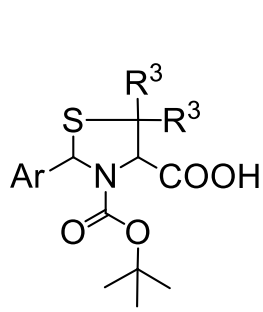

93

$76-87 \%$

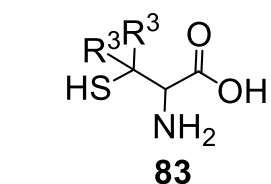

1) Stiring

2) $(\mathrm{Boc})_{2} \mathrm{O}$
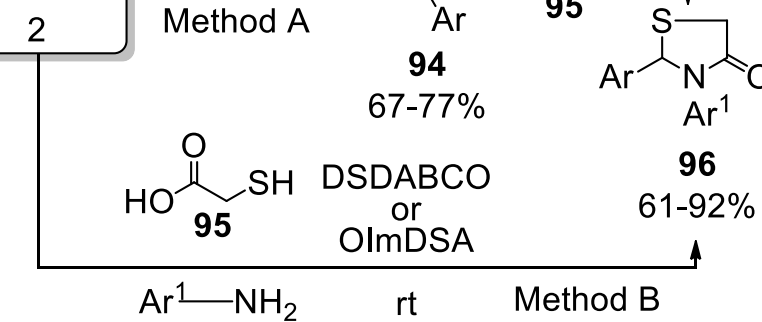

77

$\mathrm{R}^{1}=\mathrm{C}_{6} \mathrm{H}_{5}$, 5-Bromothiophen-2-yl; $\mathrm{R}^{2}=\mathrm{C}_{6} \mathrm{H}_{5}, 4-\mathrm{H}_{3} \mathrm{C}-\mathrm{C}_{6} \mathrm{H}_{4}, 4-\mathrm{Cl}_{-} \mathrm{C}_{6} \mathrm{H}_{4}, 3-\mathrm{O}_{2} \mathrm{~N}-\mathrm{C}_{6} \mathrm{H}_{4}, 4-\mathrm{HO}-\mathrm{C}_{6} \mathrm{H}_{4} ; \mathrm{R}^{3}=\mathrm{H}, \mathrm{CH}_{3} ; \mathrm{Ar}^{1}=4-$ $\mathrm{H}_{3} \mathrm{CO}-\mathrm{C}_{6} \mathrm{H}_{4}, 2-\mathrm{H}_{3} \mathrm{C}-4-\mathrm{O}_{2} \mathrm{~N}-\mathrm{C}_{6} \mathrm{H}_{3}, \mathrm{C}_{6} \mathrm{H}_{5}, 4-\mathrm{O}_{2} \mathrm{~N}-\mathrm{C}_{6} \mathrm{H}_{4}, 4-\mathrm{H}_{3} \mathrm{C}-\mathrm{C}_{6} \mathrm{H}_{4}$, 4-Fluorobenzyl, 4-(4-Chlorophenyl)thiazol-2-yl.

Scheme 40. Synthesis of 2-pyrazole-3-phenyl-1,3-thiazolidine-4-ones 96.

Visagaperumal et al. $^{70}$ reported that thiazolidin-4-ones 96 has been synthesized by stirring of pyrazole4-carboxaldehyde $\mathbf{2}$ with 2-mercaptoacetic acid $\mathbf{9 5}$ and different substituted aromatic amines $\mathbf{7 7}$ in dry toluene under the effect of microwave heating for 12 min (Scheme 41). 


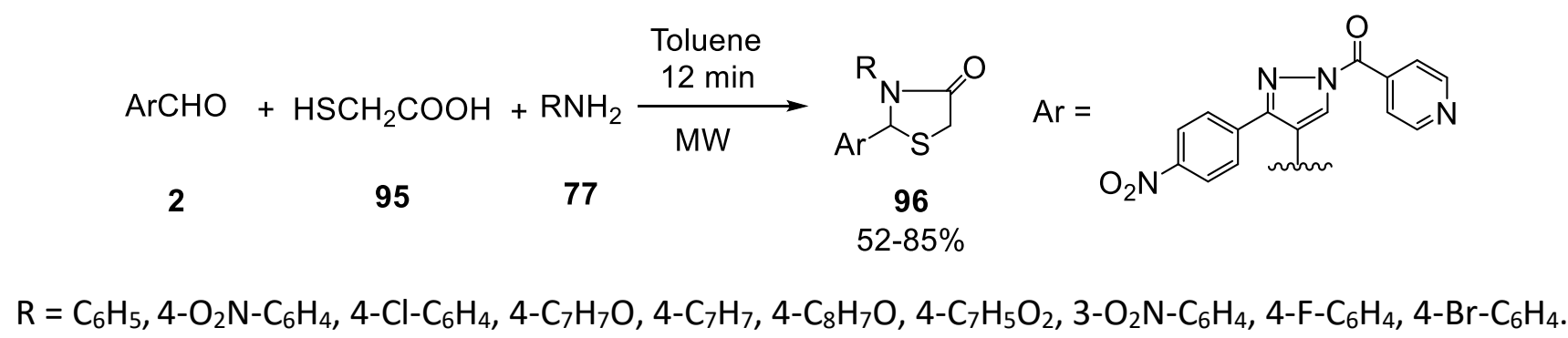

Scheme 41. Synthesis of thiazolidin-4-ones 96.

One-pot multi-component cyclocondensation of pyrazole-4-carboxaldehydes 2, thiosemicarbazide 97, and maleic anhydride 98 using baker's yeast as a catalyst afforded pyrazol-4-yl substituted thiazoles 99.140 Thiazoles 99 were also synthesized via two-steps-reactions. Thus, cyclocondensation reaction of pyrazole-4carboxaldehydes $\mathbf{2}$ with substituted thiosemicarbazide derivatives $\mathbf{9 7}$ afforded the corresponding thiosemicarbazone derivatives $\mathbf{1 0 0} 62,103,126,141-147$ which underwent cyclization with maleic anhydride $\mathbf{9 8}$ to furnish thiazole derivatives $99^{144,146}$ (Scheme 42).

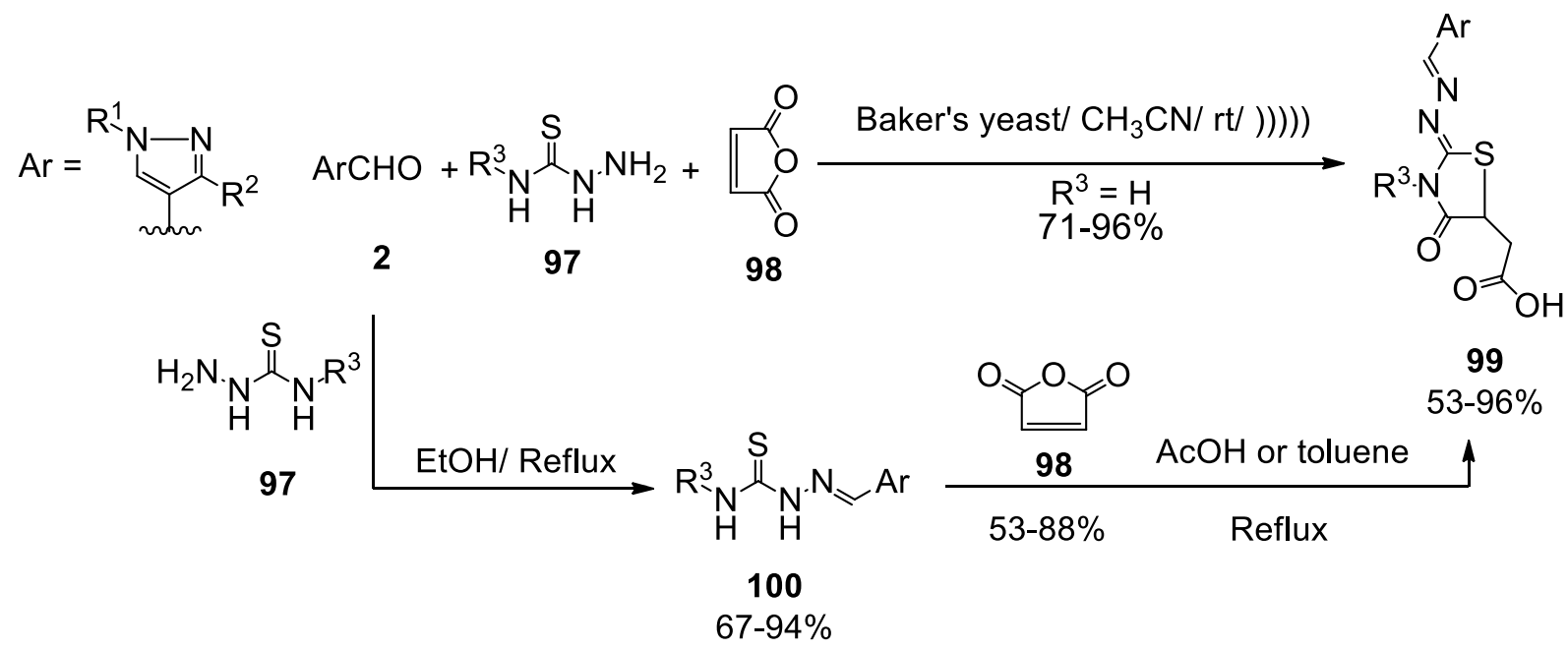

$\mathrm{R}^{1}=\mathrm{C}_{6} \mathrm{H}_{5}, 3-\mathrm{Cl}-\mathrm{C}_{6} \mathrm{H}_{4} ; \mathrm{R}^{2}=\mathrm{C}_{6} \mathrm{H}_{5}, 4-\mathrm{H}_{3} \mathrm{C}-\mathrm{C}_{6} \mathrm{H}_{4}, 4-\mathrm{Br}-\mathrm{C}_{6} \mathrm{H}_{4}, 4-\mathrm{F}-\mathrm{C}_{6} \mathrm{H}_{4}, 4-\mathrm{H}_{3} \mathrm{CO}-\mathrm{C}_{6} \mathrm{H}_{4}, 4-\mathrm{O}_{2} \mathrm{~N}-\mathrm{C}_{6} \mathrm{H}_{4} ; \mathrm{R}^{3}=\mathrm{H}, \mathrm{C}_{6} \mathrm{H}_{5}, 4-\mathrm{H}_{3} \mathrm{CO}-$ $\mathrm{C}_{6} \mathrm{H}_{4}, 4-\mathrm{F}-\mathrm{C}_{6} \mathrm{H}_{4}$, Cyclohexane.

Scheme 42. Synthesis of thiazole derivatives 99.

Nikpassand et al. ${ }^{148}$ reported that stirring a mixture of a pyrazole-carboxaldehyde 2 , thiosemicarbazide 97, bromoacetophenone 101 in the presence of [PDBMDIm] Br as a catalyst at room temperature afforded 2hydrazonyl-4-phenylthiazoles 102 (Scheme 43).

Cyclization of thiosemicarbazone derivatives 100 either by ethyl chloroacetate, ${ }^{141}$ methyl $\alpha$ bromopropionate, ${ }^{141}$ ethyl bromoacetate, ${ }^{62,142,143}$ chloroacetic acid, ${ }^{149}$ diethyl-2-bromomalonate ${ }^{146}$ or methyl bromoacetate ${ }^{126} 64$ furnished the thiazole derivatives 103 (Scheme 44).

Similarly, Some 4-arylthiazol-2-yl-hydrazines derivatives $104^{62,126,141-144,146,147}$ were prepared by reaction of the appropriate $\alpha$-haloketones 101 with the corresponding thiosemicarbazone 100 . The reaction of thiosemicarbazone derivatives $\mathbf{1 0 0}$ with the appropriate hydrazonoyl halides $\mathbf{1 0 5}$ gave 5-phenylazo-thiazol-2-yl- 
hydrazine derivative $106 .{ }^{103}$ Compounds 106 were alternatively obtained by reaction of $\omega$-bromoacetophenone 101 with 100 to give 1-((1,3-diphenyl-1H-pyrazol-4- yl)methylene)-2-(4-phenylthiazol-2-yl)hydrazine 107103 followed by reaction with benzenediazonium chloride $\mathbf{1 0 8}^{103}$ (Scheme 45).

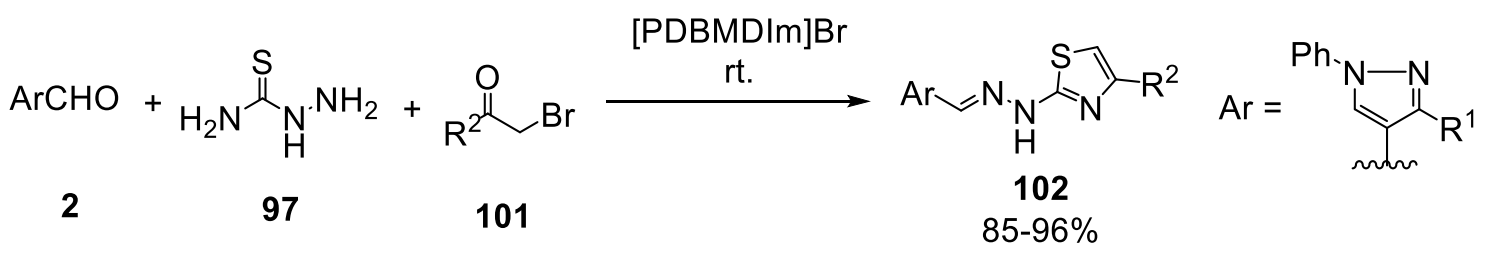

[PDBMDIm]Br = 3,3'-(pentane-1,5-diyl)bis(1,2-dimethyl-1Himidazol-3-ium)bromide $\mathrm{R}^{1}=4-\mathrm{H}_{3} \mathrm{C}-\mathrm{C}_{6} \mathrm{H}_{4}, 2-\mathrm{Cl}-\mathrm{C}_{6} \mathrm{H}_{4}, 4-\mathrm{Cl}-\mathrm{C}_{6} \mathrm{H}_{4}, \mathrm{C}_{6} \mathrm{H}_{5}, 4-\mathrm{HO}-\mathrm{C}_{6} \mathrm{H}_{4}, 4-\mathrm{HO}_{-} \mathrm{C}_{6} \mathrm{H}_{4} ; \mathrm{R}^{2}=2-\mathrm{HO}-\mathrm{C}_{6} \mathrm{H}_{4}, \mathrm{C}_{6} \mathrm{H}_{5}, 2-\mathrm{Br}^{-} \mathrm{C}_{6} \mathrm{H}_{4}, 2-\mathrm{H}_{3} \mathrm{CO}-$

$\mathrm{C}_{6} \mathrm{H}_{4}, 3-\mathrm{O}_{2} \mathrm{~N}-\mathrm{C}_{6} \mathrm{H}_{4}, 3-\mathrm{O}_{2} \mathrm{~N}-\mathrm{C}_{6} \mathrm{H}_{4}, 4-\mathrm{Cl}-\mathrm{C}_{6} \mathrm{H}_{4}, 4-\mathrm{HO}-\mathrm{C}_{6} \mathrm{H}_{4}$.

Scheme 43. Synthesis of 2-hydrazonyl-4-phenylthiazoles 102.

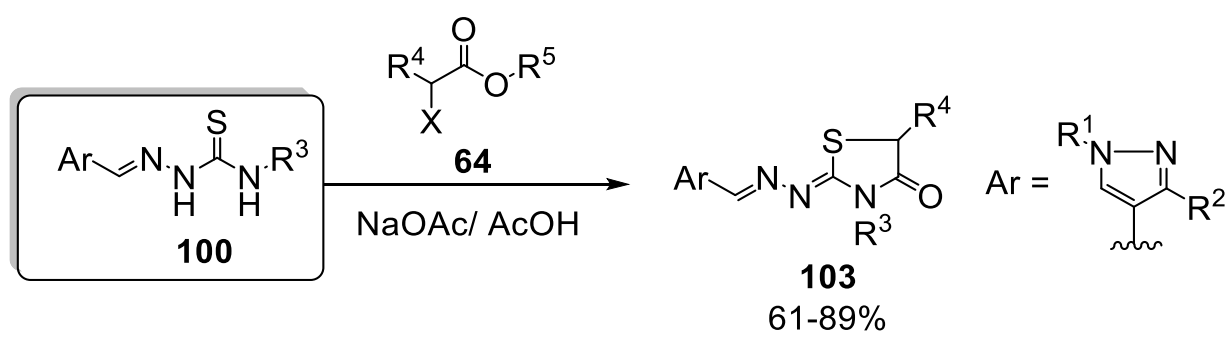

$\mathrm{R}^{1}=\mathrm{C}_{6} \mathrm{H}_{5}, 4-\mathrm{H}_{2} \mathrm{NO}_{2} \mathrm{~S}-\mathrm{C}_{6} \mathrm{H}_{4}, 3-\mathrm{Cl}-\mathrm{C}_{6} \mathrm{H}_{4} ; \mathrm{R}^{2}=\mathrm{C}_{6} \mathrm{H}_{5}, 4-\mathrm{Cl}-\mathrm{C}_{6} \mathrm{H}_{4}, 4-\mathrm{Br}-\mathrm{C}_{6} \mathrm{H}_{4}, 4-\mathrm{Cl}_{2} \mathrm{C}_{6} \mathrm{H}_{4}, 4-\mathrm{H}_{3} \mathrm{CO}-\mathrm{C}_{6} \mathrm{H}_{4}$, 5-Bromothiophen2-yl; $\mathrm{R}^{3}=\mathrm{H}, \mathrm{C}_{6} \mathrm{H}_{5}, 4-\mathrm{H}_{3} \mathrm{C}-\mathrm{C}_{6} \mathrm{H}_{4}, 4-\mathrm{Cl}-\mathrm{C}_{6} \mathrm{H}_{4}, 4-\mathrm{F}-\mathrm{C}_{6} \mathrm{H}_{4}, 4-\mathrm{H}_{3} \mathrm{CO}-\mathrm{C}_{6} \mathrm{H}_{4}$, Cyclohexane; $\mathrm{R}^{4}=\mathrm{H}, \mathrm{CH}_{3}, \mathrm{COOEt} ; \mathrm{R}^{5}=\mathrm{H}, \mathrm{CH}_{3}$, $\mathrm{C}_{2} \mathrm{H}_{5} ; \mathrm{X}=\mathrm{Br}, \mathrm{Cl}$.

Scheme 44. Synthesis of thiazole derivatives 103.

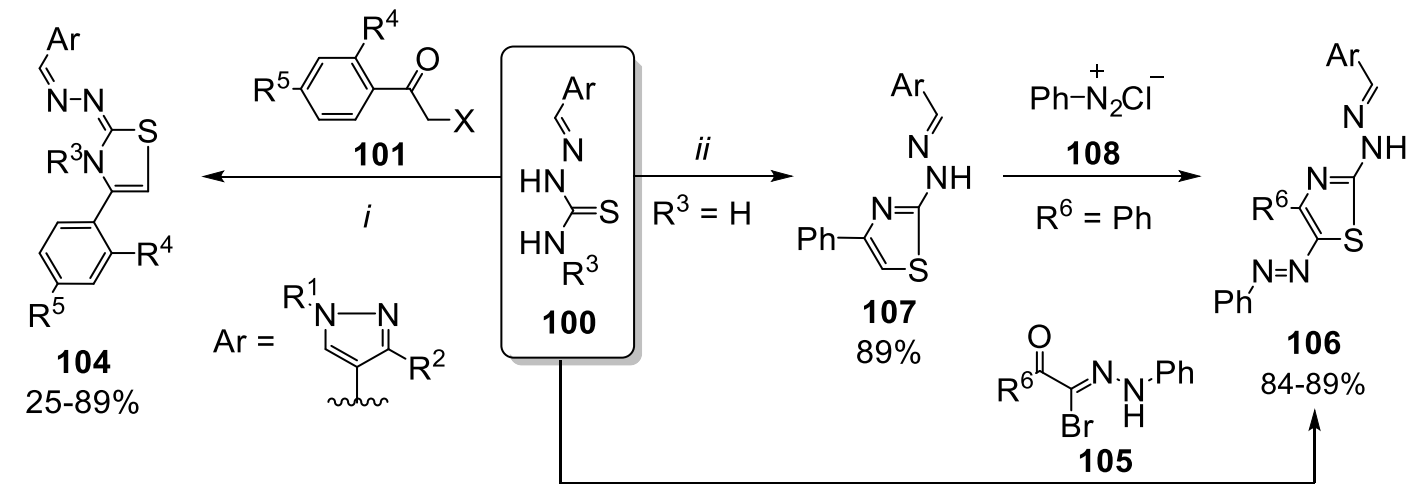

$\mathrm{R}^{1}=\mathrm{C}_{6} \mathrm{H}_{5}, 3-\mathrm{H}_{3} \mathrm{C}-\mathrm{C}_{6} \mathrm{H}_{4}, 4-\mathrm{H}_{2} \mathrm{NO}_{2} \mathrm{~S}-\mathrm{C}_{6} \mathrm{H}_{4}, 3-\mathrm{Cl}-\mathrm{C}_{6} \mathrm{H}_{4} ; \mathrm{R}^{2}=\mathrm{C}_{6} \mathrm{H}_{5}, 4-\mathrm{H}_{3} \mathrm{C}-\mathrm{C}_{6} \mathrm{H}_{4}, 4-\mathrm{H}_{3} \mathrm{CO}-\mathrm{C}_{6} \mathrm{H}_{4}, 4-\mathrm{HO}-\mathrm{C}_{6} \mathrm{H}_{4}, 4-\mathrm{O}_{2} \mathrm{~N}-\mathrm{C}_{6} \mathrm{H}_{4}, 4-$ $\mathrm{F}-\mathrm{C}_{6} \mathrm{H}_{4}, 4-\mathrm{Cl}-\mathrm{C}_{6} \mathrm{H}_{4}, 4-\mathrm{Br}-\mathrm{C}_{6} \mathrm{H}_{4}, 4-\left(\mathrm{C}_{6} \mathrm{H}_{5}-\mathrm{CH}_{2}-\mathrm{O}\right)-\mathrm{C}_{6} \mathrm{H}_{4}, 5-B r o m o t h i o p h e n-2-\mathrm{yl} ; \mathrm{R}^{3}=\mathrm{H}, \mathrm{C}_{6} \mathrm{H}_{5}, 4-\mathrm{H}_{3} \mathrm{C}-\mathrm{C}_{6} \mathrm{H}_{4}, 4-\mathrm{Cl}-\mathrm{C}_{6} \mathrm{H}_{4}, 4-$ F- $\mathrm{C}_{6} \mathrm{H}_{4}, 4-\mathrm{H}_{3} \mathrm{CO}-\mathrm{C}_{6} \mathrm{H}_{4}$, Cyclohexane; $\mathrm{R}^{4}=\mathrm{H}, \mathrm{F}, \mathrm{NO}_{2}, \mathrm{OCH}_{3}, \mathrm{OH} ; \mathrm{R}^{5}=\mathrm{H}, \mathrm{SO}_{2} \mathrm{CH}_{3}, \mathrm{~F}, \mathrm{Cl}, \mathrm{Br}, \mathrm{CH}_{3}, \mathrm{NO}_{2}, \mathrm{C}_{6} \mathrm{H}_{5} ; \mathrm{R}^{6}=\mathrm{H}$, $\mathrm{CH}_{3}, \mathrm{C}_{6} \mathrm{H}_{5}, 2-\mathrm{C}_{8} \mathrm{H}_{5} \mathrm{O} ; \mathrm{X}=\mathrm{Cl}, \mathrm{Br} ; i=\mathrm{PEG}-400 /$ stir. at r. t., ${ }^{147}$ ethanol/ sodium acetate/ reflux, ${ }^{146}$ dry ethanol/ reflux $;{ }^{142} i i=\mathrm{PhCOCH}_{2} \mathrm{Br} /$ ethanol/ sodium acetate/ reflux. ${ }^{103}$

Scheme 45. Synthesis of thiazol-2-yl-hydrazines derivatives 104 and 106. 
On the other hand, treatment of pyrazolyl hydrazone derivative $\mathbf{1 0 0}$ with thioglycolic acid $\mathbf{9 5}$ acid gave the corresponding thiazolidinedione derivative $\mathbf{1 0 9}^{145}$ (Scheme 46).

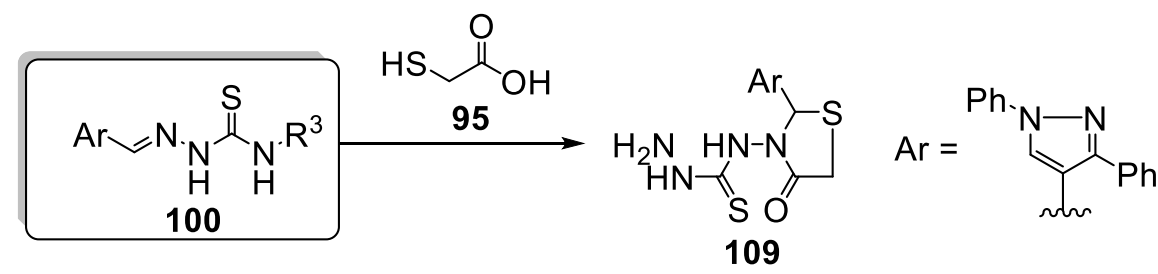

Scheme 46. Synthesis of thiazolidinedione derivatives 109.

Treatment of thiosemicarbazone derivatives 100 with ethyl bromoacetate ${ }^{142}$ or diethyl-2bromomalonate ${ }^{146} 64$ in the presence of sodium acetate led to the corresponding thiazolidinediones $\mathbf{1 1 0}^{142,146}$ (Scheme 47).

Gaffer et al. ${ }^{150}$ reported that condensation of pyrazolin-5-one derivative 111 with Pyrazolecarboxaldehyde $\mathbf{2}$ in acetic acid and fused sodium acetate yielded the corresponding condensation product 112 which underwent further heterocyclization reaction with bromoacetone and phenacyl bromide 101 to give the corresponding thiazolyl-pyrazole derivatives 113 (Scheme 48).

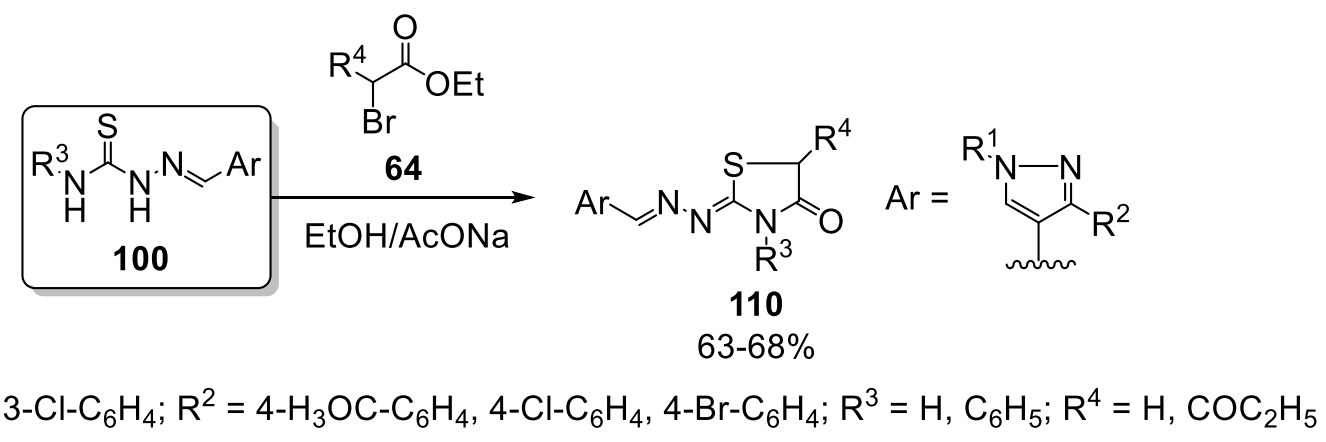

Scheme 47. Synthesis of thiazolidinediones 110.

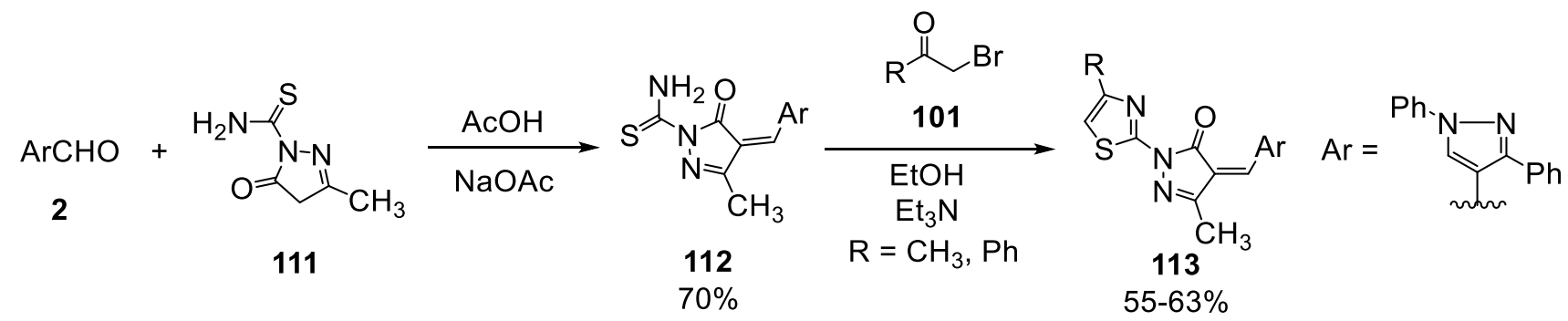

Scheme 48. Synthesis of thiazolyl-pyrazole derivatives 113.

Treatment of pyrazole-carboxaldehyde $\mathbf{2}$ with malonic acid hydrazide $\mathbf{1 1 4}$ afforded pyrazolyl malonohydrazone derivative $\mathbf{1 1 5}$ which reacted with $p$-chlorobenzaldehyde $\mathbf{4 3}$ to give the corresponding benzylidene derivative $\mathbf{1 1 6}$. The reaction of $\mathbf{1 1 6}$ with thioglycolic acid $\mathbf{9 5}$ gave the dithiazolidinone derivative $117^{145}$ (Scheme 49). 


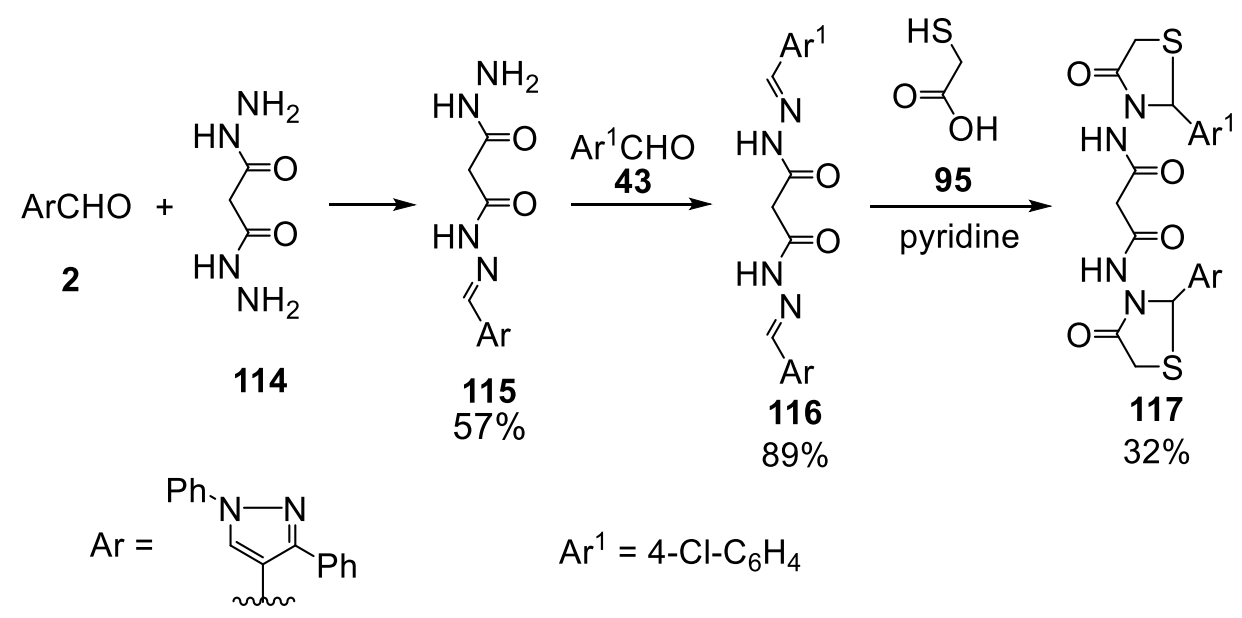

Scheme 49. Synthesis of dithiazolidinone derivative 117.

Bhatt and Sharma ${ }^{130}$ reported the synthesis of tri-substituted thiazoles derivatives $\mathbf{1 1 9}$ by the reaction of benzil 76 with pyrazole-4-carboxaldehydes $\mathbf{2}$ and ammonium thiocyanate 118 (Scheme 50).

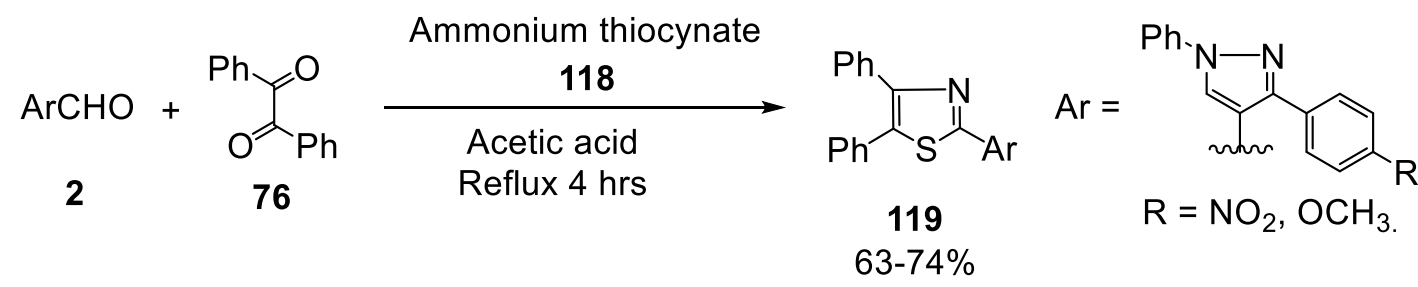

Scheme 50. Synthesis of tri-substituted thiazoles derivatives 119.

Bekhit et al. ${ }^{136}$ reported that 3-aryl-1-phenyl-1H-pyrazole-4-aldoximes 121 were obtained by the condensation of 3-aryl-1-phenyl-1H-pyrazole-4-carboxaldehydes 2 with hydroxylamine hydrochloride 120 in ethanol containing anhydrous sodium acetate. Dehydration of the oximes 121 with acetic anhydride $\left(\mathrm{Ac}_{2} \mathrm{O}\right)$ afforded the cyano derivatives 122. Cyclization of the cyano derivatives 122 with cysteamine hydrochloride 123 in the presence of sodium hydroxide afforded thiazolidine derivatives 124 (Scheme 51).

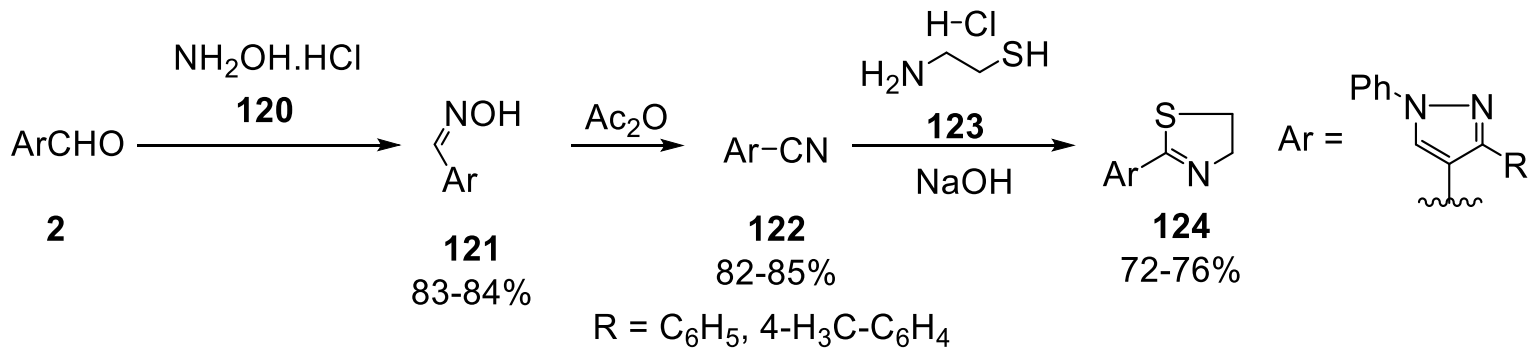

Scheme 51. Synthesis of thiazolidine derivatives 124.

3.1.2.6. 1,2-Oxaphosphole derivatives. Ali ${ }^{117}$ reported that heating of 3-(1,3-diphenyl-1H-pyrazol-4-yl)-1phenylprop-2-en-1-one $\mathbf{4 2}{ }^{116}$ with diethylphosphite 125 in boron trifluoride etherate $\left(\mathrm{BF}_{3}\right.$. $\left.\mathrm{Et}_{2} \mathrm{O}\right)$ afforded 4-(2ethoxy-2-oxido-5-phenyl-2,3-dihydro-1,2-oxaphosphol-3-yl)-1,3-diphenyl-1H-pyrazole 126 (Scheme 52). 


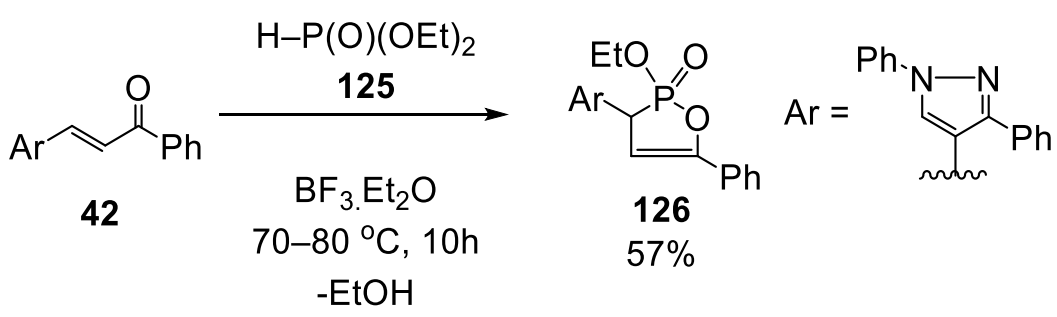

Scheme 52. Synthesis of 1,3-diphenyl-1H-pyrazole 126.

3.1.3. Monocyclic five-membered with three heteroatoms. 3.1.3.1. 1,3,4-Thiadiazole derivatives. Heating of thiosemicarbazone derivatives $\mathbf{1 0 0}$ in acetic anhydride at reflux gave the corresponding 3-acetyl-2,3-dihydro-

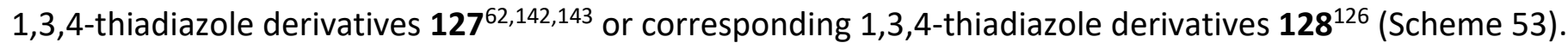

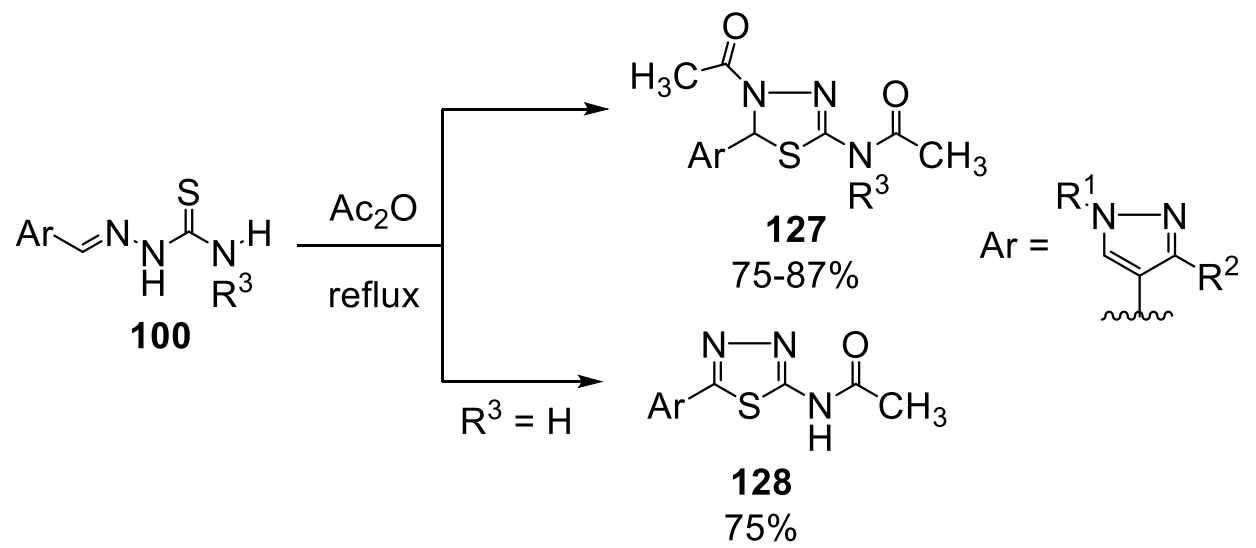

$\mathrm{R}^{1}=\mathrm{C}_{6} \mathrm{H}_{5}, 4-\left(\mathrm{H}_{2} \mathrm{NO}_{2} \mathrm{~S}\right)-\mathrm{C}_{6} \mathrm{H}_{4} ; \mathrm{R}^{2}=4-\mathrm{Cl}-\mathrm{C}_{6} \mathrm{H}_{4}, 4-\mathrm{Br}-\mathrm{C}_{6} \mathrm{H}_{4}, 4-\mathrm{H}_{3} \mathrm{CO}-\mathrm{C}_{6} \mathrm{H}_{4}, \mathrm{C}_{6} \mathrm{H}_{5}, 4-\left(\mathrm{H}_{5} \mathrm{C}_{6} \mathrm{H}_{2} \mathrm{CO}\right)-\mathrm{C}_{6} \mathrm{H}_{4} ; \mathrm{R}^{3}=\mathrm{H}, \mathrm{C}_{6} \mathrm{H}_{5}, 4-\mathrm{Cl}-$ $\mathrm{C}_{6} \mathrm{H}_{4}$.

Scheme 53. Synthesis of 1,3,4-thiadiazole derivatives 128.

Oxidative cyclization of the thiosemicarbazones 100 afforded the corresponding 1,3,4-thiaoxadiazoles $128^{151,143}$ (Scheme 54).

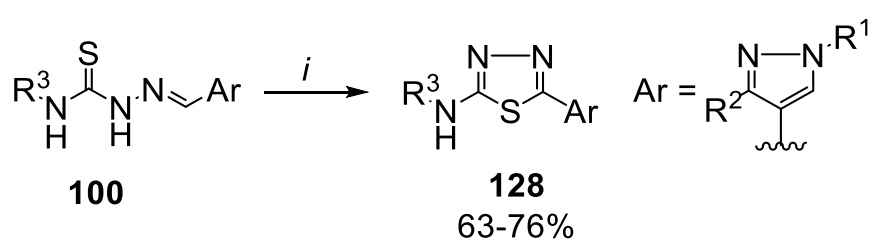

$\mathrm{R}^{1}=\mathrm{C}_{6} \mathrm{H}_{5}, 2-\mathrm{H}_{3} \mathrm{CO}-\mathrm{C}_{6} \mathrm{H}_{4}, 2-\mathrm{H}_{3} \mathrm{C}-\mathrm{C}_{6} \mathrm{H}_{4}, 4-\mathrm{Cl}-\mathrm{C}_{6} \mathrm{H}_{4}, 2,4-\mathrm{di}\left(\mathrm{O}_{2} \mathrm{~N}\right)-\mathrm{C}_{6} \mathrm{H}_{3}, 4-\left(\mathrm{H}_{2} \mathrm{NO}_{2} \mathrm{~S}\right)-\mathrm{C}_{6} \mathrm{H}_{4} ; \mathrm{R}^{2}=\mathrm{C}_{6} \mathrm{H}_{5}, 7-\mathrm{HO}-4-\mathrm{H}_{3} \mathrm{C}-2-\mathrm{oxO}-$ $2 \mathrm{H}$-chromen-8-yl; $\mathrm{R}^{3}=\mathrm{H}, \mathrm{C}_{6} \mathrm{H}_{5}, 4-\mathrm{Cl}_{-} \mathrm{C}_{6} \mathrm{H}_{4} ; i=\mathrm{Br}_{2} / \mathrm{CH}_{3} \mathrm{COOH},{ }^{151} \mathrm{FeCl}_{3} /$ (Dioxane/ ethanol)/ Reflux. ${ }^{143}$

Scheme 54. Synthesis of 1,3,4-thiaoxadiazoles 128.

Abou Elmagd et al. ${ }^{123}$ reported that heating of thiosemicarbazide $67^{124}$ with phosphoryl trichloride at reflux afforded 1,3,4-thiadiazole derivatives 129 (Scheme 55). 
<smiles>O=C(NNC(=S)Nc1ccccc1)C(=C[Te])NC(=O)c1ccccc1</smiles>

67<smiles>ClCc1ccccc1</smiles><smiles>CC=NCC</smiles>

129

$76 \%$

Scheme 55. Synthesis of 1,3,4-thiadiazole derivatives 129.

Treatment of 1,3-diphenyl-1H-pyrazole-4-carboxaldehyde $\mathbf{2}$ with the appropriate methyl(benzyl)carbodithioate 130 in 2-propanol gave the corresponding alkyl carbodithioates 131 . The reaction of the appropriate hydrazonoyl halides $\mathbf{1 0 5}$ with alkyl carbodithioates $\mathbf{1 3 1}$ in ethanol containing triethylamine (TEA) afforded 2,3-dihydro-1,3,4-thiadiazoles 132. Compounds 132 were alternatively obtained by the reaction of ethyl 2-hydrazono-3-phenyl-1,3,4-thidiazoline-5-carboxylate 133 with pyrazole-carboxaldehyde $\mathbf{2}^{103}$ (Scheme 56).

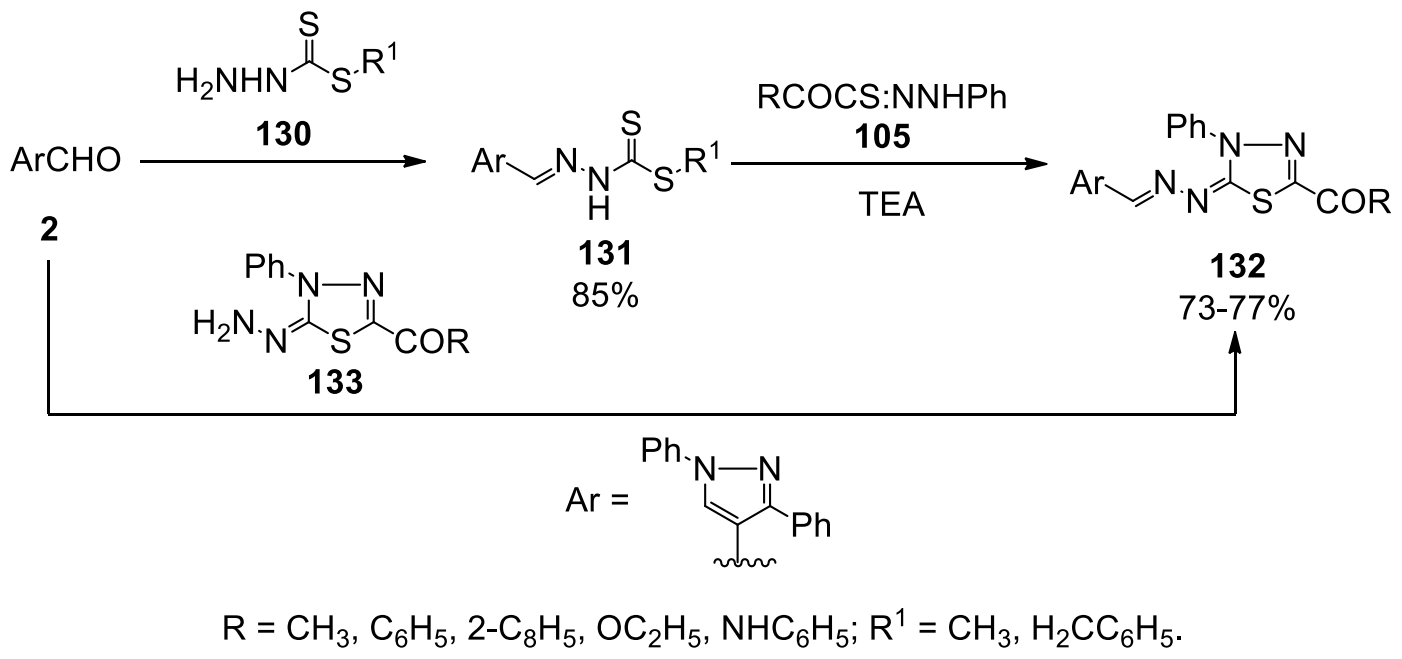

Scheme 56. Synthesis of 2,3-dihydro-1,3,4-thiadiazoles 132.

3.1.3.2. 1,2,3-Triazole derivatives. Dayakar et $a l^{152}$ reported that reduction of pyrazole-carboxaldehydes 2 using sodium borohydride $\left(\mathrm{NaBH}_{4}\right)$ provided the corresponding alcohols 134 which was converted to the corresponding azides $\mathbf{1 3 5}$ in the presence of diphenyl phosphoryl azide (DPPA) and 1,8diazabicyclo[5.4.0] undec-7-ene (DBU). Reaction of azides 135 with propargyl alcohol 87, phenyl acetylenes 137 and dimethyl/ diethyl acetylene dicarboxylate $\mathbf{1 3 9}$ in the presence of copper(II) sulfate pentahydrate $\left(\mathrm{CuSO}_{4} .5 \mathrm{H}_{2} \mathrm{O}\right) /$ sodium ascorbate in aqueous alcohol medium provided the corresponding pyrazolyl-1H-1,2,3triazolyl alcohols 136, pyrazolyl-1H-1,2,3-triazoles 138 and pyrazolyl-1H-1,2,3-triazolyl carboxylates 140, respectively (Scheme 57). 


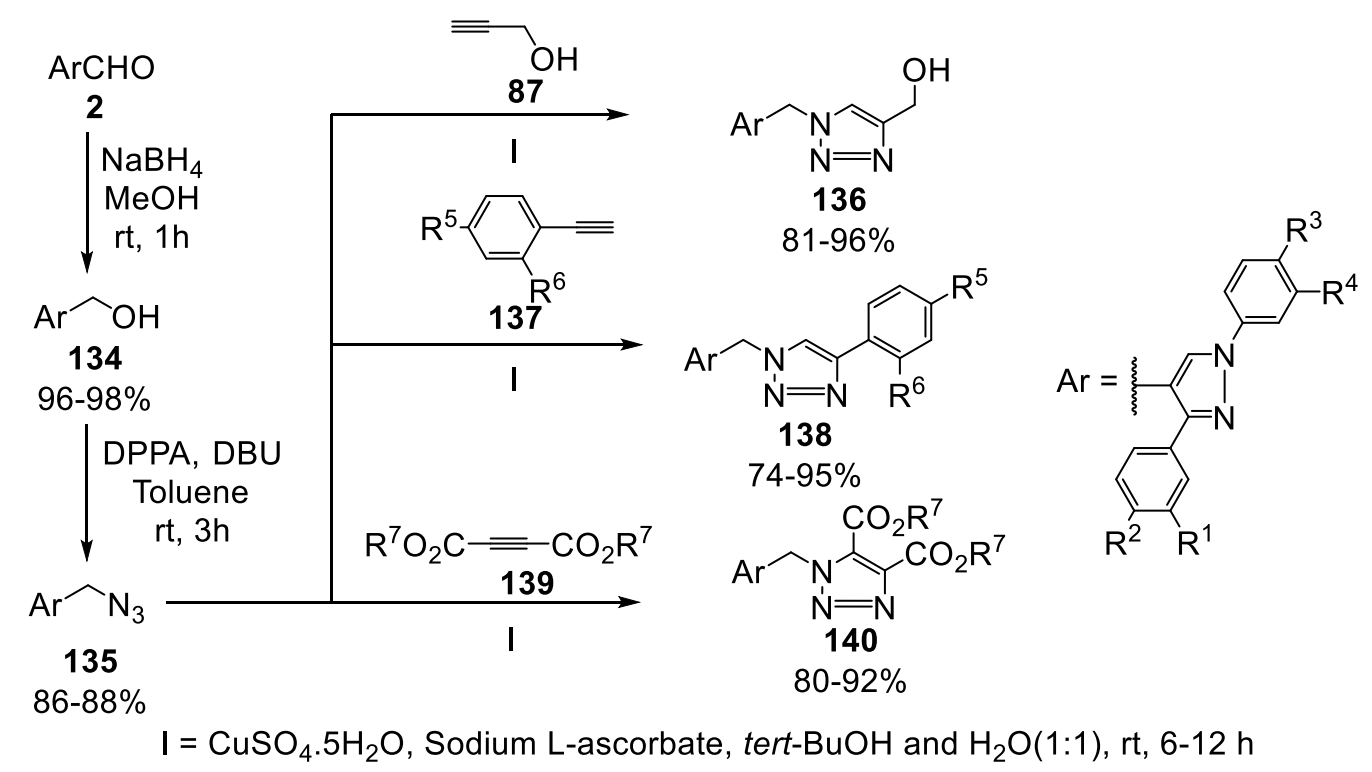

$\mathrm{R}^{1}=\mathrm{H}, \mathrm{Cl} ; \mathrm{R}^{2}=\mathrm{H}, \mathrm{Cl}, \mathrm{CH}_{3}, \mathrm{OCH}_{3} ; \mathrm{R}^{3}=\mathrm{H}, \mathrm{Cl}, \mathrm{CH}_{3} ; \mathrm{R}^{4}=\mathrm{H}, \mathrm{CH}_{3} ; \mathrm{R}^{5}=\mathrm{H}, \mathrm{CF}_{3}, \mathrm{NH}_{2}, \mathrm{~N}\left(\mathrm{CH}_{3}\right)_{2}, \mathrm{~F}, \mathrm{Cl}, \mathrm{Br}, \mathrm{CH}_{3}, \mathrm{OCH}_{3}, \mathrm{OCH}_{3} ;$ $\mathrm{R}^{6}=\mathrm{H}, \mathrm{CF}_{3}, \mathrm{CH}_{3} ; \mathrm{R}^{7}=\mathrm{CH}_{3}, \mathrm{C}_{2} \mathrm{H}_{5}$.

Scheme 57. Synthesis of pyrazolyl-1H-1,2,3-triazoles 136, 138 and 140.

The reaction of aryl azides 141 with 0 -propargylated pyrazole derivatives $\mathbf{8 8}$ afforded the corresponding 1,4-disubstituted-1,2,3-triazole-linked pyrazole hybrids $142^{134}$ (Scheme 58).

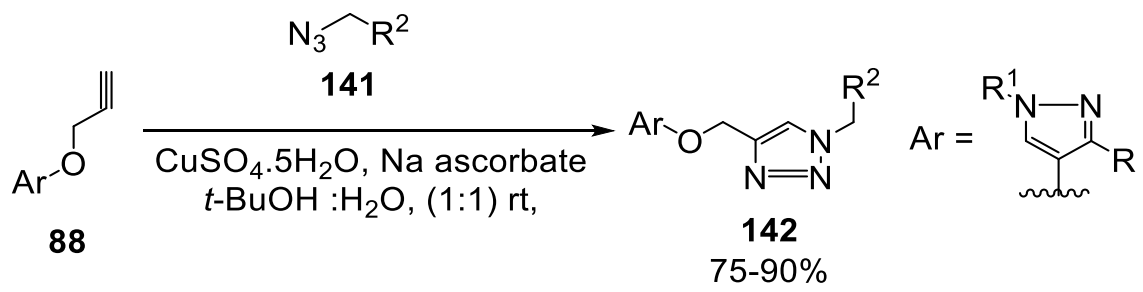

$\mathrm{R}=\mathrm{H}, \mathrm{C}_{6} \mathrm{H}_{5}, 4-\mathrm{H}_{3} \mathrm{C}-\mathrm{C}_{6} \mathrm{H}_{4}, 4-\mathrm{Cl}-\mathrm{C}_{6} \mathrm{H}_{4} ; \mathrm{R}^{1}=4-\mathrm{H}_{3} \mathrm{C}-\mathrm{C}_{6} \mathrm{H}_{4}, \mathrm{C}_{6} \mathrm{H}_{5}, 4-\mathrm{F}-\mathrm{C}_{6} \mathrm{H}_{4}, 4-\mathrm{F}-\mathrm{C}_{6} \mathrm{H}_{4} ; \mathrm{R}^{2}=\mathrm{C}_{6} \mathrm{H}_{5}, 2-\mathrm{Cl}_{-}-\mathrm{C}_{6} \mathrm{H}_{4}, 2-\mathrm{Br}-\mathrm{C}_{6} \mathrm{H}_{4}, 2-$

$\mathrm{F}-\mathrm{C}_{6} \mathrm{H}_{4}, 3-\mathrm{Cl}-\mathrm{C}_{6} \mathrm{H}_{4}, 4-\mathrm{H}_{3} \mathrm{CO}-\mathrm{C}_{6} \mathrm{H}_{4}, 4-\mathrm{O}_{2} \mathrm{~N}-\mathrm{C}_{6} \mathrm{H}_{4}$. $t$-BuOH: tert-Butyl alcohol; $\mathrm{NaH}$ : Sodium hydride.

Scheme 58. Synthesis of 1,4-disubstituted-1,2,3-triazole-linked pyrazole hybrids 142.

3.1.3.3. 1,2,4-Triazole derivatives. Heating of 1-(3-chlorophenyl)-3-(4-methoxyphenyl)-1H-pyrazole-4carboxaldehyde $\mathbf{2}$ with semicarbazide hydrochloride or thiosemicarbazide $\mathbf{9 7}$ in absolute ethanol at reflux in the presence of few drops of glacial acetic acid afforded 1,2,4-triazole-3(4H)-one $143 .{ }^{120}$ On the other hand, heating of pyrazole-carboxaldehyde $\mathbf{2}$ with thiosemicarbazide $\mathbf{9 7}$ in water as a green solvent afforded the 5-(3-aryl-1phenyl-1H-pyrazol-4-yl)-1,2,4-triazolidine-3-thiones $144^{111}$ (Scheme 59). 


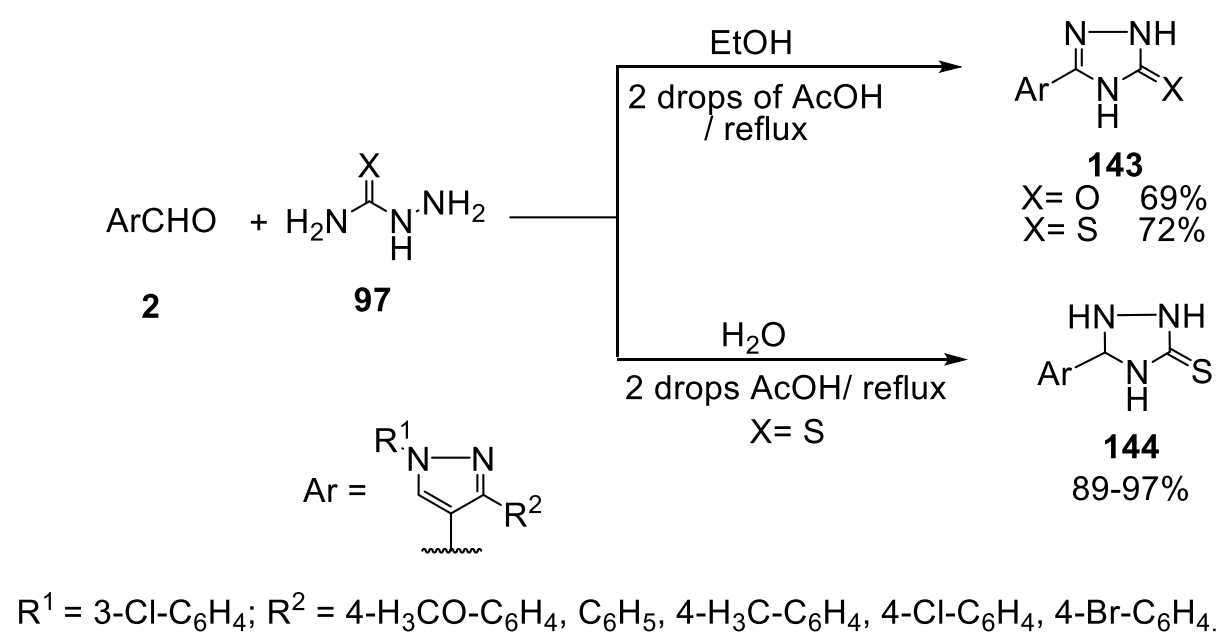

Scheme 59. Synthesis of 5-(3-aryl-1-phenyl-1H-pyrazol-4-yl)-1,2,4-triazolidine-3-thiones 144.

3.1.3.4. 1,3,4-Oxadiazole derivatives. Renuka et al. ${ }^{151}$ reported that the oxidative cyclization of semicarbazones 100 using bromine as an oxidant in acetic acid at room temperature yielded the corresponding 1,3,4-oxadiazoles 145 (Scheme 60).

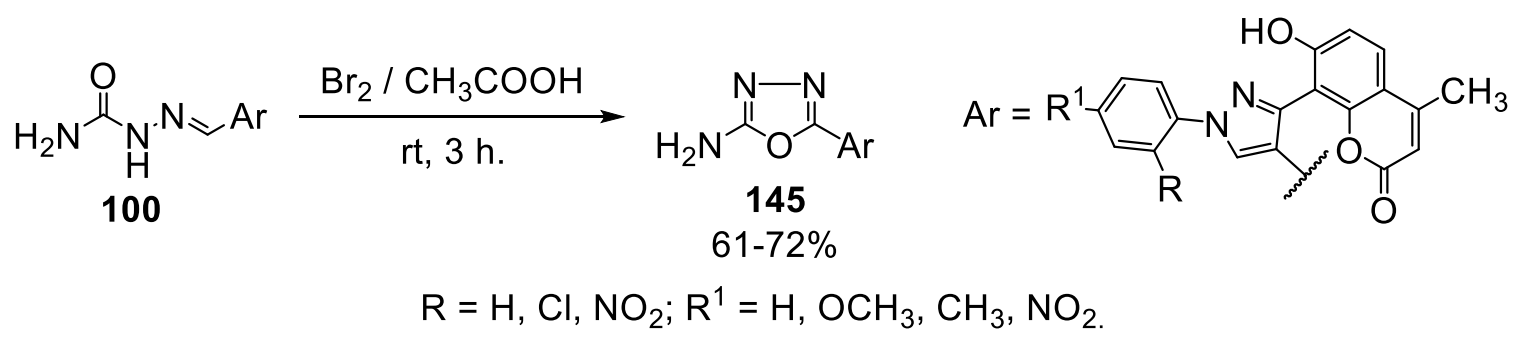

Scheme 60. Synthesis of 1,3,4-oxadiazoles 145.

Farghaly et al. ${ }^{62}$ reported the synthesis of aroylhydrazones 153 by heating the pyrazole-carboxaldehyde $\mathbf{2}$ with acid hydrazide $\mathbf{1 5 2}$ in ethanol. Cyclization of aroylhydrazones $\mathbf{1 5 3}$ upon treatment with acetic anhydride gave the corresponding oxadiazoline derivatives 154 (Scheme 61).

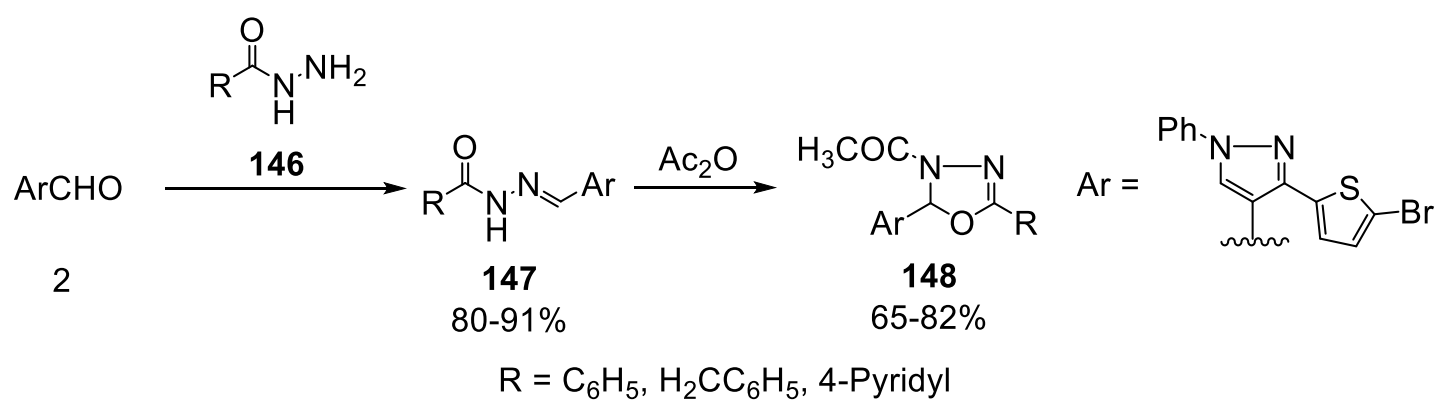

Scheme 61. Synthesis of oxadiazoline derivatives 154.

Abu-Zaied et al. ${ }^{65}$ reported that 5-(3-isobutyl-1-phenyl-1H-pyrazole-4-yl)-1,3,4-oxadiazole-2-thiol 150 was prepared by the reaction of ethanolic potassium hydroxide solution of 3-isobutyl-1-phenyl-1H-pyrazole-4carbohydrazide 149 with carbon disulfide $\left(\mathrm{CS}_{2}\right)$ (Scheme 62). 


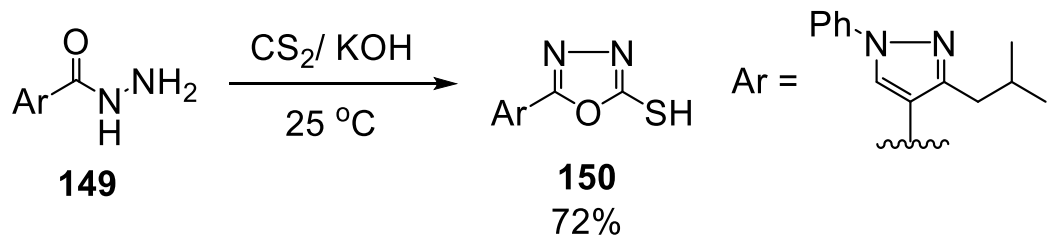

Scheme 62. Synthesis of 5-(3-isobutyl-1-phenyl-1H-pyrazole-4-yl)-1,3,4-oxadiazole-2-thiol 150.

Abou Elmagd et al. ${ }^{123}$ reported that heating of thiosemicarbazide derivative 67 in glacial acetic acid at reflux afforded the oxazolone derivative 151 (Scheme 63).

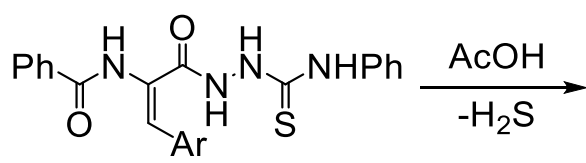

67<smiles>O=C(N/C(=C/Br)c1nnc(Nc2ccccc2)o1)c1ccccc1</smiles>

151 $41 \%$

Scheme 63. Synthesis of oxazolone derivative 151.

3.1.4. Monocyclic six-membered with one heteroatom. 3.1.4.1. Pyran derivatives. Heating of 2-((1,3diphenylpyrazol-4-yl)methylene)malononitrile 54 with ethyl acetoacetate $\mathbf{6 4}$ in methylene chloride at reflux in the presence of triethylamine gave the ethyl 6-amino-5-cyano-4-(1,3-diphenylpyrazol-4-yl)-2-methyl-4H-pyran3-carboxylate derivative $152^{141}$ (Scheme 64).

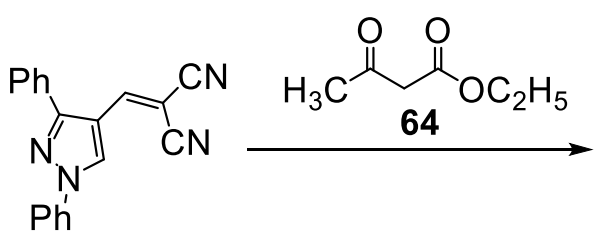

54<smiles>CCOCC1=C(C)OC(N)=C(C#N)C1c1cn(-c2ccccc2)nc1-c1ccccc1</smiles><smiles>CC(C)c1cnn(-c2ccccc2)c1-c1ccccc1</smiles> 

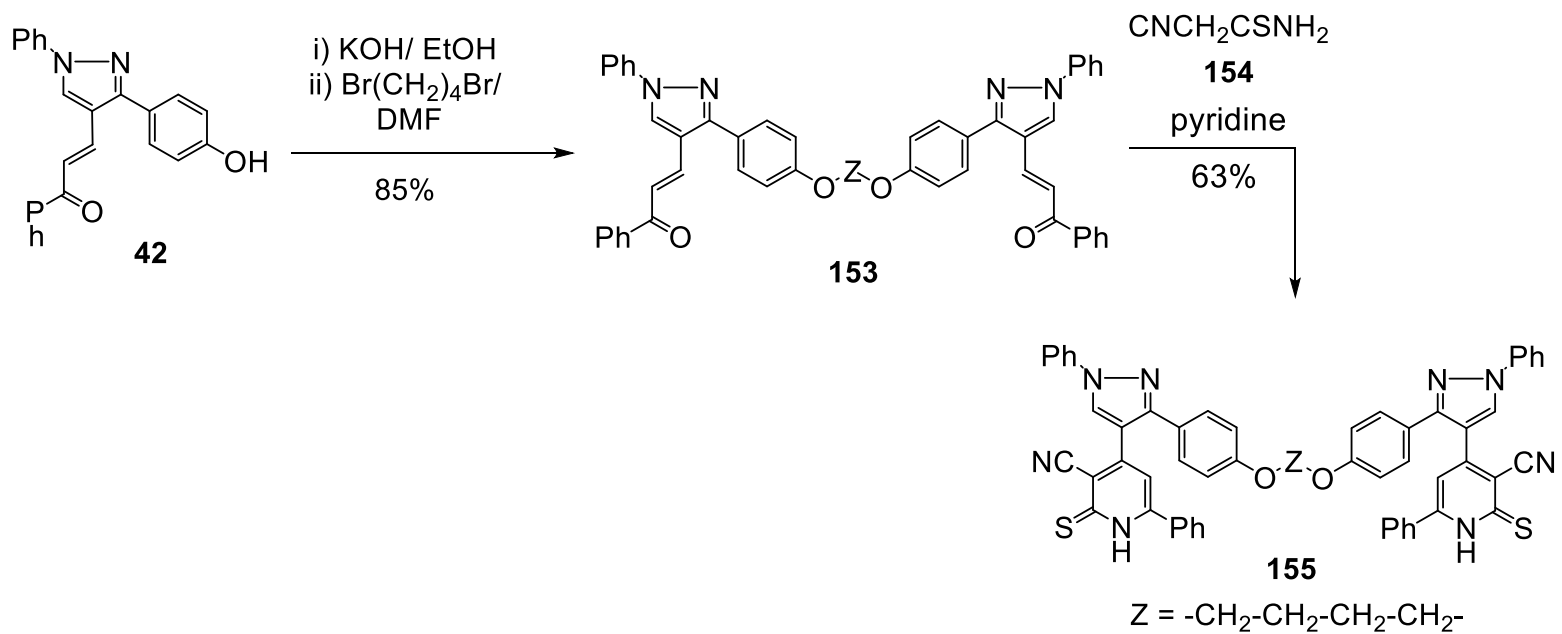

Scheme 65. Synthesis of bis(pyridine-2(1H)-thione) derivative 155.

The reaction of $\alpha, b$-unsaturated ketones $\mathbf{4 2}$ with malononitrile $\mathbf{5 3}$ in ethanol at reflux in the presence of ammonium acetate afforded the corresponding 2-amino-3-cyano-pyridine derivatives $156 .{ }^{112,114,154}$ On the other hand, a reaction of $\mathbf{4 2}$ with $\mathbf{5 3}$ in the presence of either sodium methoxide/ methanol or sodium ethoxide/ ethanol gave the corresponding 2-alkoxynicotinonitriles $157^{155}$ (Scheme 66).

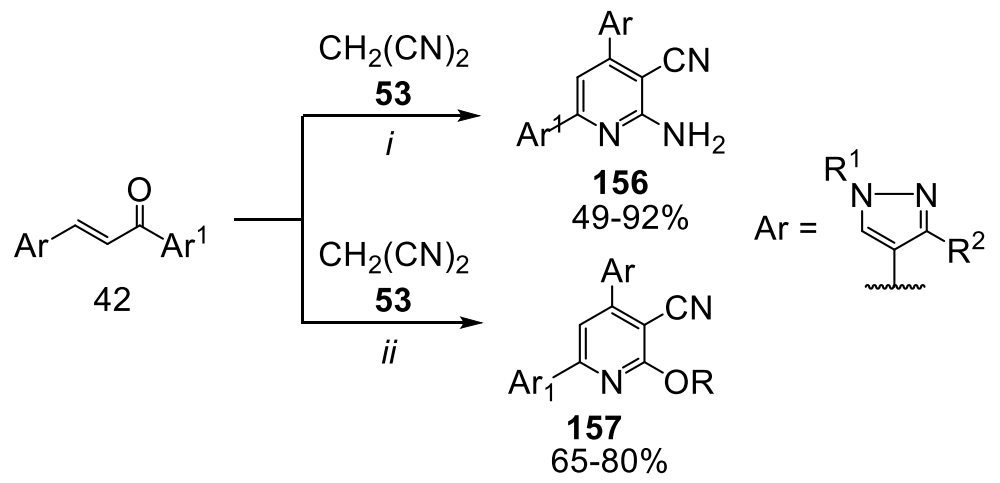

$\mathrm{R}^{1}=\mathrm{C}_{6} \mathrm{H}_{5}, 3-\mathrm{Cl}-\mathrm{C}_{6} \mathrm{H}_{4} ; \mathrm{R}^{2}=\mathrm{C}_{6} \mathrm{H}_{5}, 4-\mathrm{H}_{3} \mathrm{CO}-\mathrm{C}_{6} \mathrm{H}_{4} ; \mathrm{Ar}^{1}=\mathrm{C}_{6} \mathrm{H}_{5}$, Benzofuran-2-yl, 2-Thienyl, 4-Br- $\mathrm{C}_{6} \mathrm{H}_{4}, 4-\mathrm{H}_{3} \mathrm{CO}-\mathrm{C}_{6} \mathrm{H}_{4} ; \mathrm{R}^{3}$ $=\mathrm{CH}_{3}, \mathrm{C}_{2} \mathrm{H}_{5} ; \boldsymbol{i}=$ Grinding $92 \%,{ }^{112}$ traditional $75 \%,{ }^{112} \mathrm{CH}_{3} \mathrm{COONH}_{4} /$ ethanol $/ \mathrm{reflux} ;{ }^{114,154} \boldsymbol{i i}=\mathrm{RONa} / \mathrm{ROH} /$ stirring/ r.t..

Scheme 66. Synthesis of 2-amino-3-cyano-pyridine derivatives 156 and 2-alkoxynicotinonitriles 157.

Heterocyclization of chalcones carrying pyrazole $\mathbf{4 2}$ with ethyl cyanoacetate $\mathbf{6 4}$ and ammonium acetate gave the corresponding 2-oxo-1,2-dihydropyridine-3-carbonitrile 158 . $^{112,114,135,141,156,157}$ On the other hand, heating chalcones 3 with cynothioacetamide 154 in ethanol at reflux afforded the corresponding 3-cyanopyridine-2(1H)-thiones 159. ${ }^{112,141,153,157}$ Moreover, reaction of chalcones $\mathbf{4 2}$ with 2-cyanoacetohydrazide $\mathbf{5 7}$ afforded 1-amino-2-oxo-1,2-dihydropyridine-3-carbonitrile $\mathbf{1 6 0}^{112}$ (Scheme 67). 


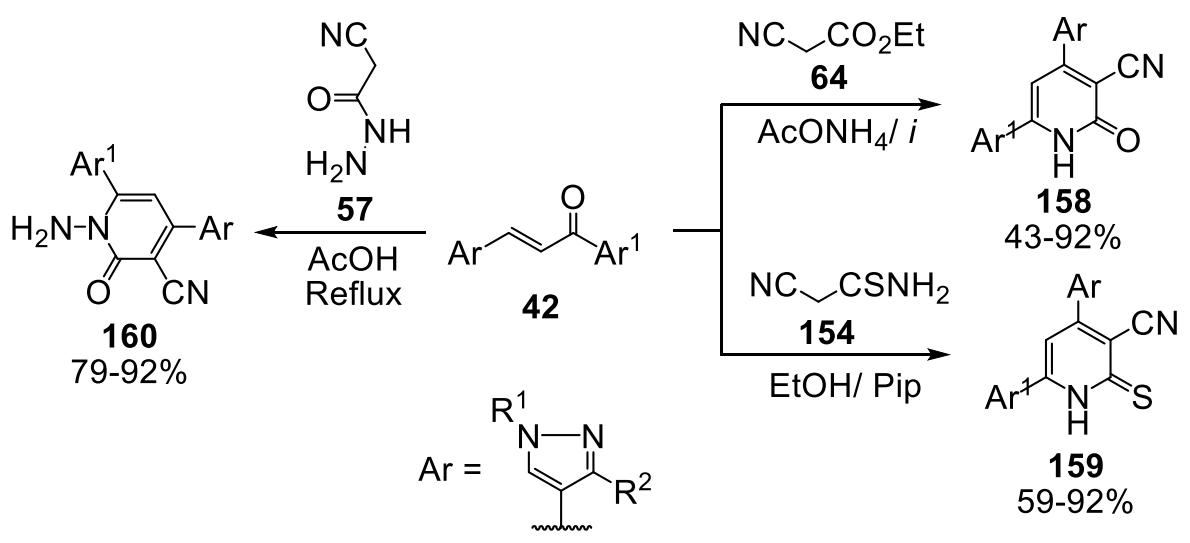

$\mathrm{R}^{1}=4-\mathrm{Cl}-\mathrm{C}_{6} \mathrm{H}_{4}, 4-\mathrm{H}_{3} \mathrm{C}-\mathrm{C}_{6} \mathrm{H}_{4}, \mathrm{C}_{6} \mathrm{H}_{5}, 3-\mathrm{Cl}-\mathrm{C}_{6} \mathrm{H}_{4} ; \mathrm{R}^{2}=\mathrm{C}_{6} \mathrm{H}_{5}, 2-\mathrm{H}_{3} \mathrm{CO}-\mathrm{C}_{6} \mathrm{H}_{4}, 4-\mathrm{H}_{3} \mathrm{CO}-\mathrm{C}_{6} \mathrm{H}_{4}, 4-\mathrm{H}_{3} \mathrm{C}-\mathrm{C}_{6} \mathrm{H}_{4}, 4-\mathrm{HO}_{6}-\mathrm{C}_{6} \mathrm{H}_{4} ; \mathrm{Ar}^{1}=$ $\mathrm{C}_{6} \mathrm{H}_{5}, 4-\mathrm{H}_{2} \mathrm{~N}-\mathrm{C}_{6} \mathrm{H}_{4}, 4-\mathrm{Cl}-\mathrm{C}_{6} \mathrm{H}_{4}, 4-\mathrm{Br}_{-} \mathrm{C}_{6} \mathrm{H}_{4}, 4-\mathrm{F}-\mathrm{C}_{6} \mathrm{H}_{4}, 2-\mathrm{HO}-\mathrm{C}_{6} \mathrm{H}_{4}, 4-\mathrm{HO}-\mathrm{C}_{6} \mathrm{H}_{4}, 4-\mathrm{H}_{3} \mathrm{CO}-\mathrm{C}_{6} \mathrm{H}_{4}, 4-\mathrm{H}_{3} \mathrm{C}-\mathrm{C}_{6} \mathrm{H}_{4}, 3-\mathrm{O}_{2} \mathrm{~N}-\mathrm{C}_{6} \mathrm{H}_{4}$, $4-\mathrm{O}_{2} \mathrm{~N}-\mathrm{C}_{6} \mathrm{H}_{4}$, 2-Thienyl, Benzofuran-2-yl; $\boldsymbol{i}=$ ethanol/ reflux, ${ }^{114,135,156} \mathrm{AcOH} /$ reflux ${ }^{112}$ Oil path $\left(150{ }^{\circ} \mathrm{C}\right) .{ }^{141,157}$

Scheme 67. Synthesis of 2-oxo-1,2-dihydropyridine-3-carbonitrile 158 and 3-cyano-pyridine-2(1H)-thiones 159.

Heating of $\alpha, b$-unsaturated ketones 42 with different phenacyl pyridium bromides 101 in acetic acid at reflux in the presence of ammonium acetate under Kröhnke's conditions gave the pyridinyl pyrazoles $\mathbf{1 6 1}^{158-160}$ (Scheme 68).

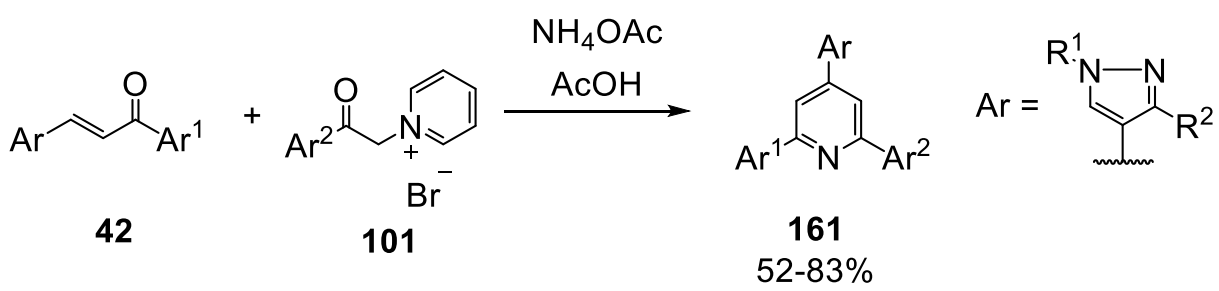

$\mathrm{R}^{1}=2,4-\mathrm{di}-\mathrm{F}-\mathrm{C}_{6} \mathrm{H}_{3}, \mathrm{C}_{6} \mathrm{H}_{5} ; \mathrm{R}^{2}=4-\mathrm{Br}_{-} \mathrm{C}_{6} \mathrm{H}_{4}, \mathrm{C}_{6} \mathrm{H}_{5}, 4-\mathrm{H}_{3} \mathrm{CO}-\mathrm{C}_{6} \mathrm{H}_{4}, 4-\mathrm{H}_{3} \mathrm{C}-\mathrm{C}_{6} \mathrm{H}_{4} ; \mathrm{Ar}^{1}=\mathrm{C}_{6} \mathrm{H}_{5}, 4-\mathrm{Br}-\mathrm{C}_{6} \mathrm{H}_{4}, 4-\mathrm{H}_{3} \mathrm{C}-\mathrm{C}_{6} \mathrm{H}_{4}, 4-\mathrm{F}-$ $\mathrm{C}_{6} \mathrm{H}_{4}, 4-\mathrm{Cl}-\mathrm{C}_{6} \mathrm{H}_{4}, 4-\mathrm{H}_{3} \mathrm{CO}-\mathrm{C}_{6} \mathrm{H}_{4}, 2-0 x 0-2 \mathrm{H}$-chromen-3-yl, 8- $\mathrm{H}_{3} \mathrm{CO}-2-0 x o-2 \mathrm{H}$-chromen-3-yl; $\mathrm{Ar}^{2}=\mathrm{C}_{6} \mathrm{H}_{5}, 4-\mathrm{Cl}_{-} \mathrm{C}_{6} \mathrm{H}_{4}, 4-$ Methyl-2-oxo-3-phenyl-2H-chromen-6-yl, 8- ${ }_{3} \mathrm{C}-2$-oxo-2H-chromen-3-yl, 8-Br-2-oxo-2H-chromen-3-yl, 2-oxo$2 \mathrm{H}$-chromen-3-yl, 8- $\mathrm{H}_{3} \mathrm{CO}-2-0 x \mathrm{o}-2 \mathrm{H}$-chromen-3-yl, 3-oxo-3H-benzo[f]chromen-2-yl.

Scheme 68. Synthesis of pyridinyl pyrazoles 161.

3.1.4.2.2. Synthesis of pyridine derivatives from aryliden malononitrile carrying pyrazole. The reaction of pyrazol-4-ylmethylene-malononitrile 54 and 2-cyanoacetamide or 2-cyanothioacetamide 154 in sodium ethoxide at reflux afforded 4,6-diamino-5-((1-(3-chlorophenyl)-3-(4-methoxyphenyl)-1H-pyrazol-4yl)methylene)-2,5-dihydro-2-oxo(thioxo)pyridine-3-carbonitriles $\mathbf{1 6 2 .}{ }^{118}$ Reaction of pyrazol-4-ylmethylenemalononitrile 54 with malononitrile dimer, ethyl cyanoacetate dimer or ethyl-3-amino-2,4-dicyanobut-2enoate 163 gave the corresponding dihydropyridine derivatives $164.161,162$ On the other hand, reaction of 54 with 2-cyanoacetohydrazide 57 in ethanol in the presence of piperidine gave 1,6-diamino-4-(1,3-diphenyl- $1 \mathrm{H}$ pyrazol-4-yl)-2-oxo-1,2-dihydropyridine-3,5-dicarbonitrile $165^{119}$ (Scheme 69). 


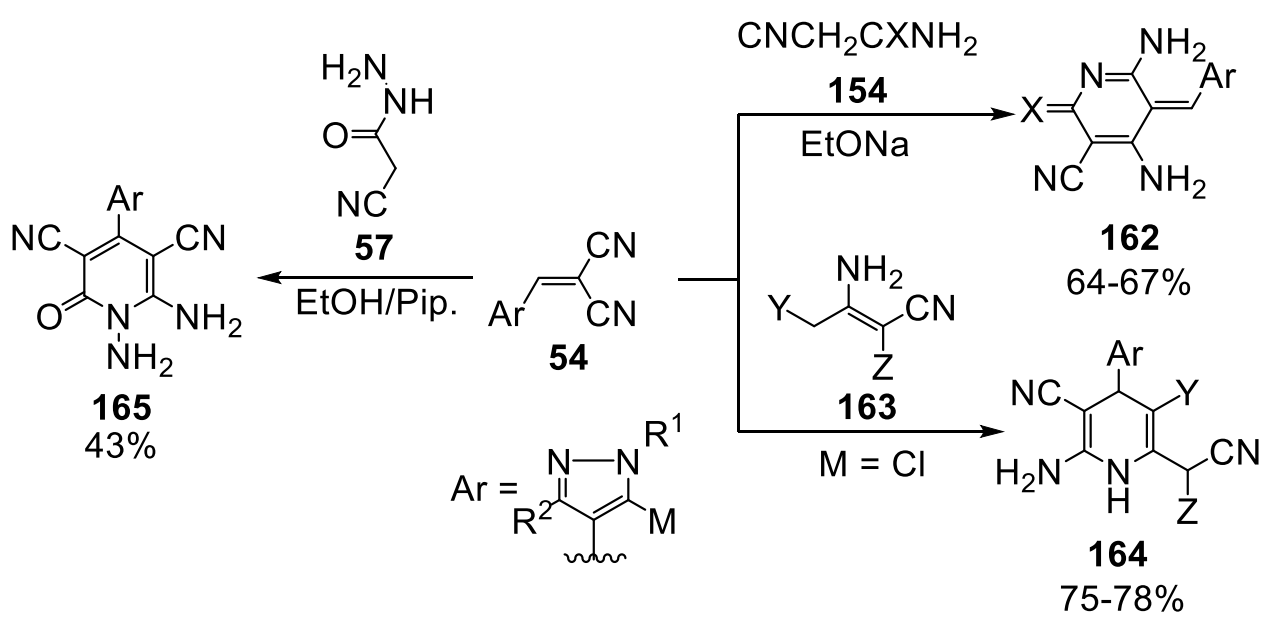

$\mathrm{R}^{1}=3-\mathrm{Cl}-\mathrm{C}_{6} \mathrm{H}_{4}, \mathrm{C}_{6} \mathrm{H}_{5} ; \mathrm{R}^{2}=4-\mathrm{H}_{3} \mathrm{CO}-\mathrm{C}_{6} \mathrm{H}_{4}, \mathrm{C}_{6} \mathrm{H}_{5}, 4-\mathrm{H}_{3} \mathrm{C}-\mathrm{C}_{6} \mathrm{H}_{4} ; \mathrm{M}=\mathrm{H}, \mathrm{Cl} ; \mathrm{X}=\mathrm{S}, \mathrm{O} ; \mathrm{Y}=\mathrm{CN}, \mathrm{CO}_{2} \mathrm{Et} ; \mathrm{Z}=\mathrm{CN}, \mathrm{CO}_{2} \mathrm{Et}$.

Scheme 69. Synthesis of dihydropyridine derivatives 162 and 164.

Condensation of pyrazol-4-ylmethylene malononitrile $\mathbf{5 4}$ with different aryl ketones $\mathbf{4 1}$ in the presence of sodium methoxide/ ethoxide gave the corresponding 2-alkyloxypyridine-3-carbonitriles $166 .{ }^{163}$ On the other hand, pyrazol-4-ylmethylene malononitrile $54^{125,164}$ could be cyclized with acetone and ammonium acetate to give the corresponding 3-amino-6-methylpyridine-2-carbonitriles $167^{125}$ (Scheme 70).

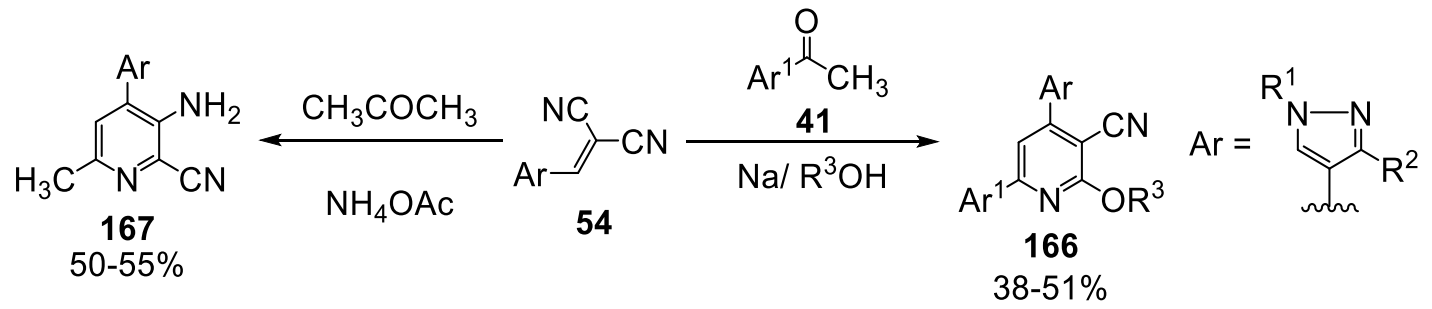

$\mathrm{R}^{1}=3-\mathrm{Cl}-\mathrm{C}_{6} \mathrm{H}_{4} ; \mathrm{R}^{2}=$ Pyren-1-yl; $\mathrm{Ar}^{1}=\mathrm{C}_{6} \mathrm{H}_{5}, 2$ 2-Thienyl, 2-Pyridinyl, 2-Furanyl, 2-Pyrrolyl; $\mathrm{R}^{3}=\mathrm{CH}_{3}, \mathrm{C}_{2} \mathrm{H}_{5}$.

Scheme 70. Synthesis of pyridine-2/3-carbonitriles 166 and 167.

3.1.4.2.3. Synthesis of pyridine derivatives from ethyl arylidencyanoacetate linked to pyrazole moiety. The Knoevenagel condensation reaction of pyrazole-4-carboxaldehyde 2 with ethyl cyanoacetate 64 gave ethyl-2cyano-acrylate derivative 168 which was then reacted with acetophenone 41 to afford 2-oxo-6-phenyl-1,2dihydro-pyridine-3-carbonitrile $\mathbf{1 6 9}^{165}$ (Scheme 71).

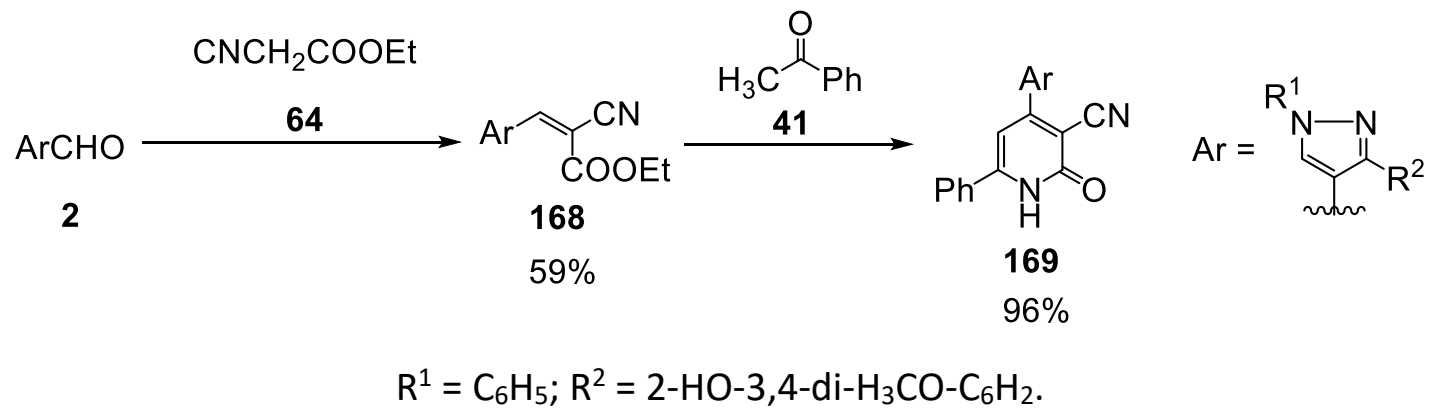

Scheme 71. Synthesis of 2-oxo-6-phenyl-1,2-dihydro-pyridine-3-carbonitrile 169. 
3.1.4.2.4. Synthesis of pyridine derivatives from hydrazone carrying pyrazole. Treatment of hydrazone derivative 58 with ethyl-2-cyano-3-arylacrylate 170 yielded pyridinone $171 .{ }^{166}$ On the other hand, the reaction of 58 with 2-arylidenemalononitrile derivatives 172 afforded 6-amino-2-oxo-1,2-dihydropyridine-3,5dicarbonitriles $173^{167,168}$ (Scheme 72 ).

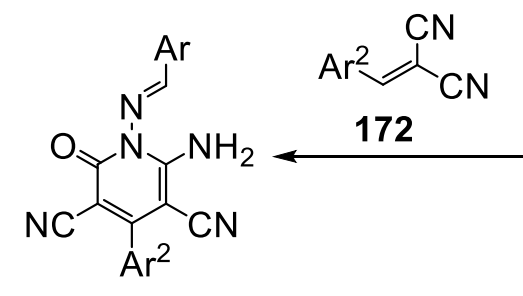

173

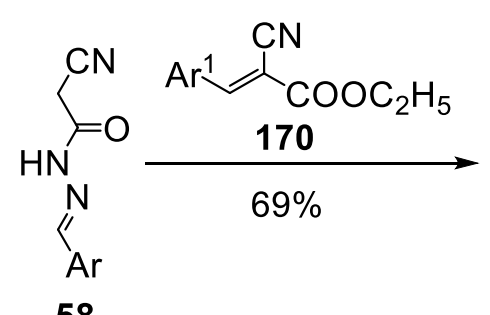

58<smiles>N#Cc1c(Br)c(C#N)c(=O)n(/N=C/[Ba])c1O</smiles>

171<smiles></smiles>

$\mathrm{R}^{1}=\mathrm{C}_{6} \mathrm{H}_{5}, 3-\mathrm{Cl}-\mathrm{C}_{6} \mathrm{H}_{4} ; \mathrm{R}^{2}=\mathrm{C}_{6} \mathrm{H}_{5}, 4-\mathrm{H}_{3} \mathrm{CO}-\mathrm{C}_{6} \mathrm{H}_{4} ; \mathrm{Ar}^{1}=4-\mathrm{F}-\mathrm{C}_{6} \mathrm{H}_{4} ; \mathrm{Ar}^{2}=\mathrm{C}_{6} \mathrm{H}_{5}, 2-\mathrm{HO}-\mathrm{C}_{6} \mathrm{H}_{4}, 4-\mathrm{HO}-\mathrm{C}_{6} \mathrm{H}_{4}, 2-\mathrm{Cl}_{-}-\mathrm{C}_{6} \mathrm{H}_{4}, 3-\mathrm{Cl}-$ $\mathrm{C}_{6} \mathrm{H}_{4}, 4-\mathrm{C} 1-\mathrm{C}_{6} \mathrm{H}_{4}, 2-\mathrm{F}-\mathrm{C}_{6} \mathrm{H}_{4}, 3-\mathrm{F}-\mathrm{C}_{6} \mathrm{H}_{4}, 4-\mathrm{F}-\mathrm{C}_{6} \mathrm{H}_{4}, 4-\mathrm{H}_{3} \mathrm{C}-\mathrm{C}_{6} \mathrm{H}_{4}, 4-\mathrm{H}_{3} \mathrm{CO}-\mathrm{C}_{6} \mathrm{H}_{4}, 3,4,5-\left(\mathrm{H}_{3} \mathrm{CO}\right)_{3}-\mathrm{C}_{6} \mathrm{H}_{2}, 2-\mathrm{O}_{2} \mathrm{~N}-\mathrm{C}_{6} \mathrm{H}_{4}, 3-\mathrm{O}_{2} \mathrm{~N}-$ $\mathrm{C}_{6} \mathrm{H}_{4}, 4-\mathrm{O}_{2} \mathrm{~N}-\mathrm{C}_{6} \mathrm{H}_{4}, 2-\mathrm{Br}-\mathrm{C}_{6} \mathrm{H}_{4}, 3-\mathrm{Br}-\mathrm{C}_{6} \mathrm{H}_{4}, 4-\mathrm{Br}-\mathrm{C}_{6} \mathrm{H}_{4}, 2-\mathrm{F}_{3} \mathrm{C}-\mathrm{C}_{6} \mathrm{H}_{4}, 4-\mathrm{F}_{3} \mathrm{C}-\mathrm{C}_{6} \mathrm{H}_{4}$, 4-iso-Propyl- $\mathrm{C}_{6} \mathrm{H}_{4}$.

Scheme 72. Synthesis of pyridinones 171 and 173.

3.1.4.2.5. Synthesis of pyridine derivatives from reaction of pyrazole-carboxaldehyde with 3aminocrotononitrile. The pseudo-multicomponent reaction of 1,3-diphenyl-1H-pyrazole-4-carboxaldehyde 2 with two equivalent of 3-aminocrotononitrile 174 in glacial acetic acid afforded 3,5-dicyano-2,6-dimethyl-1,4dihydropyridines $\mathbf{1 7 5 .} .^{169}$ On the other hand, the one-pot multicomponent reaction of pyrazole-4carboxaldehyde 2, 3-aminocrotononitrile 174 and ethyl acetoacetate (EAA) or/ methyl acetoacetate (MAA) 64 in the presence of $\mathrm{Fe}^{+3}$ montmorillonite clay $\mathrm{K}-10$ or $\mathrm{HY}$-zeolite under microwave irradiation in aqueous medium afforded the corresponding 1,4-dihydropyridine derivatives $176^{170}$ (Scheme 73).

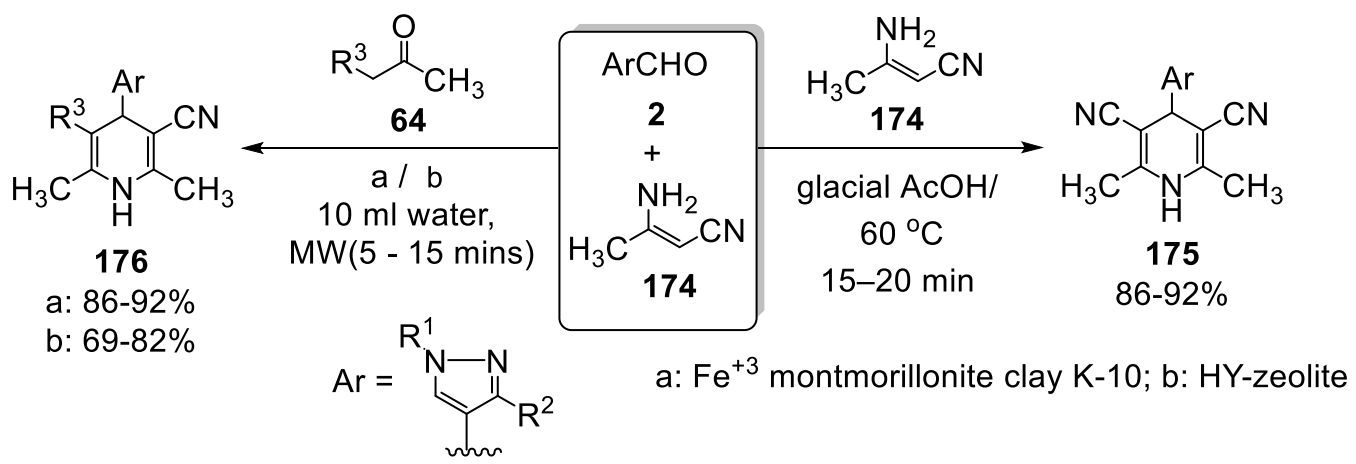

$\mathrm{R}^{1}=\mathrm{C}_{6} \mathrm{H}_{5} ; \mathrm{R}^{2}=\mathrm{C}_{6} \mathrm{H}_{5}, 4-\mathrm{H}_{3} \mathrm{C}-\mathrm{C}_{6} \mathrm{H}_{4}, 4-\mathrm{Cl}-\mathrm{C}_{6} \mathrm{H}_{4}, 4-\mathrm{O}_{2} \mathrm{~N}-\mathrm{C}_{6} \mathrm{H}_{4}, 2-\mathrm{HO}-\mathrm{C}_{6} \mathrm{H}_{4}, 2-\mathrm{H}_{3} \mathrm{CO}-\mathrm{C}_{6} \mathrm{H}_{4}, 3-\mathrm{O}_{2} \mathrm{~N}-\mathrm{C}_{6} \mathrm{H}_{4}, 4-\mathrm{F}-\mathrm{C}_{6} \mathrm{H}_{4}, 4-\mathrm{Br}-$

$\mathrm{C}_{6} \mathrm{H}_{4}, 2-\mathrm{HO}-\mathrm{C}_{6} \mathrm{H}_{4}, 4-\mathrm{F}_{3} \mathrm{C}-\mathrm{C}_{6} \mathrm{H}_{4}, 3-\mathrm{Cl}_{-} \mathrm{C}_{6} \mathrm{H}_{4}, 4-\mathrm{H}_{3} \mathrm{CO}-\mathrm{C}_{6} \mathrm{H}_{4}, 3-\mathrm{HO}-\mathrm{C}_{6} \mathrm{H}_{4} ; \mathrm{R}^{3}=\mathrm{COOCH}_{3}, \mathrm{COOC}_{2} \mathrm{H}_{5}$.

Scheme 73. Synthesis of 1,4-dihydropyridine derivatives $\mathbf{1 7 5}$ and $\mathbf{1 7 6 .}$ 
3.1.4.2.6. Synthesis of pyridine derivatives via one-pot reaction of pyrazole-carboxaldehyde. One-pot reaction of pyrazole-4-carboxaldehyde $\mathbf{2}$ with an appropriate aromatic ketone $\mathbf{4 1}$ and malononitrile $\mathbf{5 3}$ in the presence of ammonium acetate $\mathbf{7 8}$ furnished 2-amino-nicotinonitrile $\mathbf{1 7 7}^{120,155,171,172}$ (Scheme 74).

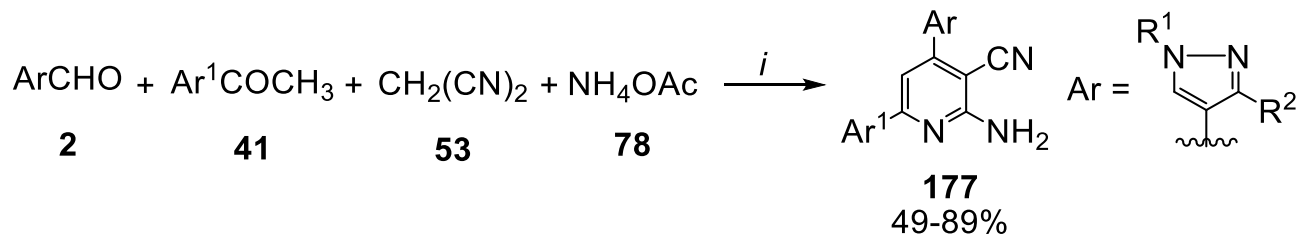

$\mathrm{R}^{1}=\mathrm{C}_{6} \mathrm{H}_{5}, 3-\mathrm{Cl}-\mathrm{C}_{6} \mathrm{H}_{4} ; \mathrm{R}^{2}=\mathrm{C}_{6} \mathrm{H}_{5}, 4-\mathrm{H}_{3} \mathrm{CO}-\mathrm{C}_{6} \mathrm{H}_{4}, 4-\mathrm{H}_{3} \mathrm{C}-\mathrm{C}_{6} \mathrm{H}_{4}$, Pyren-1-yl, 4-Cl- $\mathrm{C}_{6} \mathrm{H}_{4}, 4-\mathrm{O}_{2} \mathrm{~N}_{-} \mathrm{C}_{6} \mathrm{H}_{4}, 2-\mathrm{HO}_{6}-\mathrm{C}_{6} \mathrm{H}_{4}, 2-\mathrm{CH}_{3} \mathrm{O}-$ $\mathrm{C}_{6} \mathrm{H}_{4}, 3-\mathrm{O}_{2} \mathrm{~N}-\mathrm{C}_{6} \mathrm{H}_{4}, 4-\mathrm{F}-\mathrm{C}_{6} \mathrm{H}_{4} 4-\mathrm{OH}-\mathrm{C}_{6} \mathrm{H}_{4}, 3-\mathrm{O}_{2} \mathrm{~N}-\mathrm{C}_{6} \mathrm{H}_{4}, 4-\mathrm{H}_{2} \mathrm{~N}-\mathrm{C}_{6} \mathrm{H}_{4} ; \mathrm{Ar}^{1}=\mathrm{C}_{6} \mathrm{H}_{5}, 4-\mathrm{Cl}-\mathrm{C}_{6} \mathrm{H}_{4}, 4-\mathrm{HO}-\mathrm{C}_{6} \mathrm{H}_{4}, 4-\mathrm{H}_{3} \mathrm{C}-\mathrm{C}_{6} \mathrm{H}_{4}$, 2-Thienyl, 2-F- $\mathrm{C}_{6} \mathrm{H}_{4}, 2-\mathrm{H}_{3} \mathrm{CO}-\mathrm{C}_{6} \mathrm{H}_{4}, 2-\mathrm{Br}_{-} \mathrm{C}_{6} \mathrm{H}_{4}, 2-\mathrm{O}_{2} \mathrm{~N}-\mathrm{C}_{6} \mathrm{H}_{4}, 4-\mathrm{HO}-2-0 x \mathrm{O}-2 \mathrm{H}$-chromen-3-yl, 4-HO-8- $\mathrm{H}_{3} \mathrm{C}-2-\mathrm{oxO}-2 \mathrm{H}-$ chromen-3-yl, 4-HO-5,8-di- $\mathrm{H}_{3} \mathrm{C}-2$-oxo-2H-chromen-3-yl, (4- $\mathrm{H}_{3} \mathrm{C}-2-0 x \mathrm{H}-2 \mathrm{H}$-chromen-7-yl)oxy, Benzofuran-2-yl. i $=$ Ethanol/ reflux, ${ }^{120,155}$ ( $\mathrm{Fe}^{+3} \mathrm{~K}-10$ clay or $\mathrm{HY}$-zeolite) $\mathrm{MW}$ irradiation, ${ }^{171} \mathrm{Ac}_{2} \mathrm{O},{ }^{172}$ butanol/ reflux. ${ }^{155}$

Scheme 74. Synthesis of 2-amino-nicotinonitriles 177.

On the other hand, the reaction of equimolecular amounts of 1,3-diphenyl-1H-pyrazole-4carboxaldehyde 2, appropriate aromatic ketone $\mathbf{4 1}$ and malononitrile $\mathbf{5 3}$ in the presence of aniline afforded the corresponding pyridine-3-carbonitriles $178^{155}$ (Scheme 75).

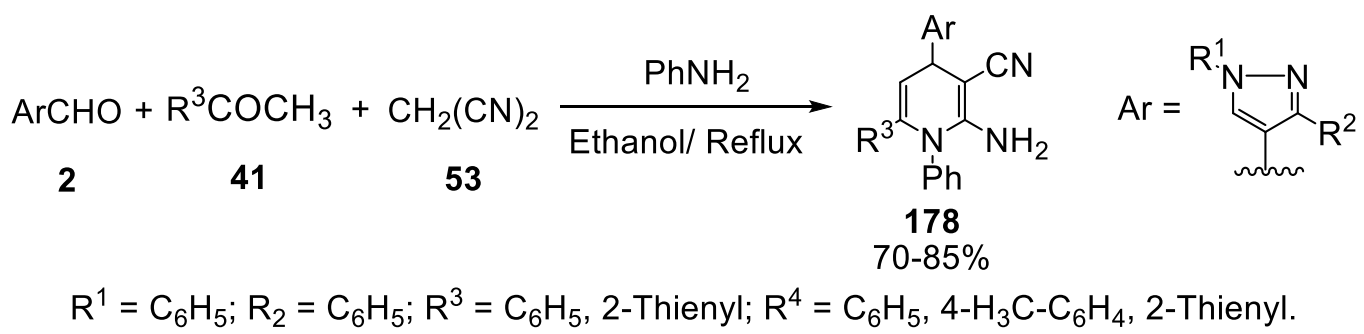

Scheme 75. Synthesis of pyridine-3-carbonitriles 178.

Heating pyrazole-4-carboxaldehyde 2 with aromatic ketone 41 and ethyl cyanoacetate 64 in the presence of ammonium acetate afforded 2-oxo-1,2-dihydropyridine-3-carbonitrile 1 179. ${ }^{120,155,173}$ Moreover, a mixture of pyrazole-4-carboxaldehyde $\mathbf{2}$ with aromatic ketone $\mathbf{4 1}$ and ethyl cyanoacetate $\mathbf{7 2}$ in the presence of aniline afforded the corresponding 2-oxo-1,2-dihydropyridine- oxo-1-phenyl-3-carbonitrile $\mathbf{1 8 0}^{155}$ (Scheme 76).

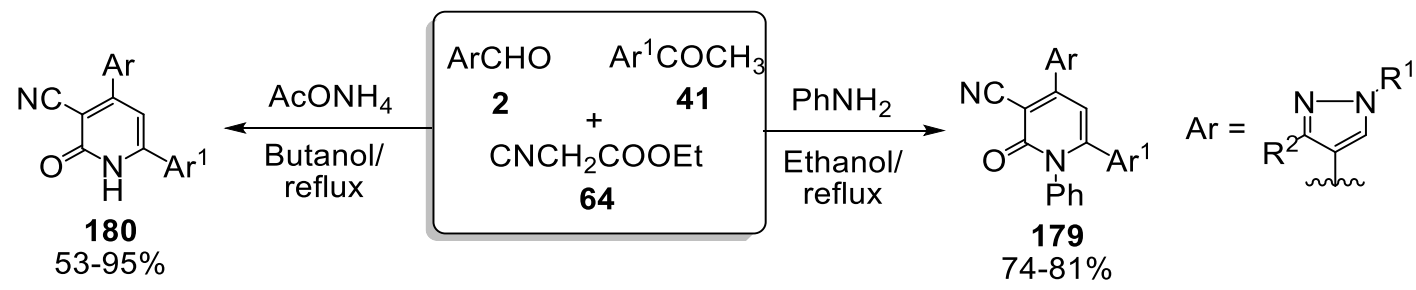

$\mathrm{R}^{1}=\mathrm{C}_{6} \mathrm{H}_{5}, 3-\mathrm{Cl}-\mathrm{C}_{6} \mathrm{H}_{4} ; \mathrm{R}^{2}=\mathrm{C}_{6} \mathrm{H}_{5}, 4-\mathrm{H}_{3} \mathrm{CO}-\mathrm{C}_{6} \mathrm{H}_{4}$, Pyren-1-yl; $\mathrm{Ar}^{1}=\mathrm{C}_{6} \mathrm{H}_{5}, 4-\mathrm{Cl}_{-}-\mathrm{C}_{6} \mathrm{H}_{4}, 4-\mathrm{HO}-\mathrm{C}_{6} \mathrm{H}_{4}, 4-\mathrm{H}_{3} \mathrm{C}-\mathrm{C}_{6} \mathrm{H}_{4}, 2-$

Thienyl, 2-F- $\mathrm{C}_{6} \mathrm{H}_{4}, 2-\mathrm{H}_{3} \mathrm{CO}-\mathrm{C}_{6} \mathrm{H}_{4}, 2-\mathrm{Br}_{-} \mathrm{C}_{6} \mathrm{H}_{4}, 2-\mathrm{O}_{2} \mathrm{~N}-\mathrm{C}_{6} \mathrm{H}_{4}$.

Scheme 76. Synthesis of 1,2-dihydropyridine-3-carbonitriles 179 and 180. 
One-pot condensation of substituted pyrazol-4-carboxaldehydes 2, two equivalents of acetophenones 41 and ammonium acetate in the presence of solid sodium hydroxide and using polyethylene glycol (PEG-400) as a green solvent ${ }^{174}$ or acetic acid ${ }^{175}$ afforded the corresponding 2,4,6-triaryl substituted pyridines (Krohnke pyridines) $181^{174,175}$ (Scheme 77 ).

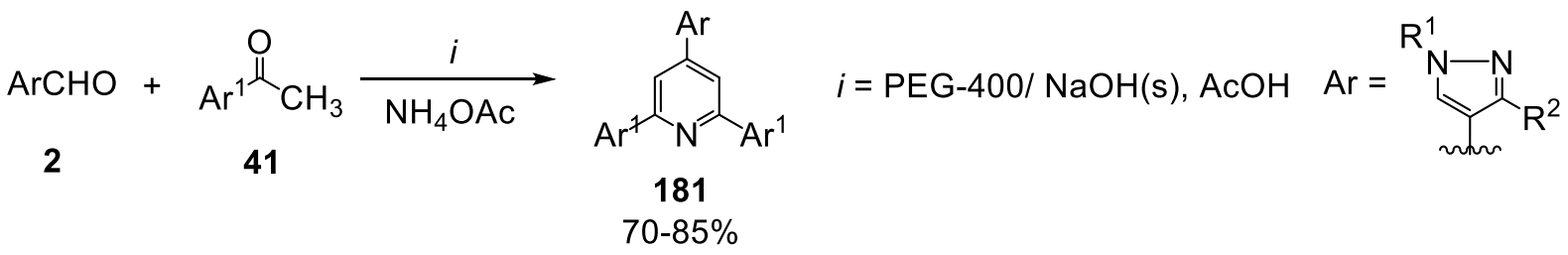

\begin{abstract}
$\mathrm{R}^{1}=\mathrm{C}_{6} \mathrm{H}_{5} ; \mathrm{R}^{2}=4-\mathrm{Cl}-\mathrm{C}_{6} \mathrm{H}_{4}, 4-\mathrm{HO}-\mathrm{C}_{6} \mathrm{H}_{4}, \mathrm{C}_{6} \mathrm{H}_{5} ; \mathrm{Ar}^{1}=5-\mathrm{Cl}-2-\mathrm{HO}-\mathrm{C}_{6} \mathrm{H}_{3}, 3-\mathrm{Br}-5-\mathrm{Cl}-2-\mathrm{HO}-\mathrm{C}_{6} \mathrm{H}_{2}, 3-\mathrm{I}-5-\mathrm{Cl}-2-\mathrm{HO}-\mathrm{C}_{6} \mathrm{H}_{2}, 3-\mathrm{I}-5-$ $\mathrm{H}_{3} \mathrm{C}-2-\mathrm{HO}-\mathrm{C}_{6} \mathrm{H}_{2}$, 3-I-4- $\mathrm{H}_{3} \mathrm{C}-2-\mathrm{HO}_{-} \mathrm{C}_{6} \mathrm{H}_{2}$, 2-Mercapto-4-methyl-1-phenyl-1H-imidazol-5-yl.
\end{abstract}

Scheme 77. Synthesis of 2,4,6-triaryl-substituted pyridines.

Under various conditions, the Hantzsch condensation reaction of pyrazole-4-carboxaldehyde 2 with 8 ketoester 64 and ammonium acetate or ammonia afforded the corresponding dihydropyridines 182. ${ }^{131,176-183}$ Similarly, $N$-aryl-1,4-dihydropyridines 183 were prepared by heating the 1,3-diphenyl-1H-pyrazole-4carboxaldehyde 2, ethyl acetoacetate/acetylacetone 64 and substituted anilines ${ }^{184}$ in methanol at reflux (Scheme 78).

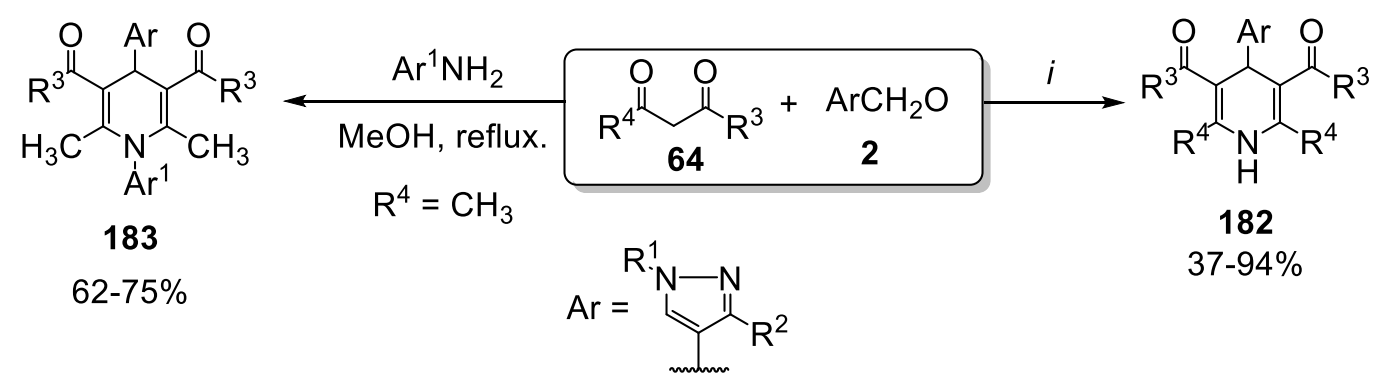

$\mathrm{R}^{1}=\mathrm{C}_{6} \mathrm{H}_{5}, \mathrm{H} ; \mathrm{R}^{2}=\mathrm{C}_{6} \mathrm{H}_{5}, 4-\mathrm{H}_{3} \mathrm{C}-\mathrm{C}_{6} \mathrm{H}_{4}, 4-\mathrm{H}_{3} \mathrm{CO}-\mathrm{C}_{6} \mathrm{H}_{4}, 4-\mathrm{F}-\mathrm{C}_{6} \mathrm{H}_{4}, 4-\mathrm{Cl}-\mathrm{C}_{6} \mathrm{H}_{4}, 4-\mathrm{Br}-\mathrm{C}_{6} \mathrm{H}_{4}, 4-\mathrm{O}_{2} \mathrm{~N}-\mathrm{C}_{6} \mathrm{H}_{4}, 3-\mathrm{H}_{3} \mathrm{CO}-\mathrm{C}_{6} \mathrm{H}_{4}, 4-$ $\mathrm{H}_{3} \mathrm{CO}_{2} \mathrm{~S}-\mathrm{C}_{6} \mathrm{H}_{4}, 2-\mathrm{O}_{2} \mathrm{~N}-\mathrm{C}_{6} \mathrm{H}_{4}, 4-\mathrm{H}_{3} \mathrm{CS}-\mathrm{C}_{6} \mathrm{H}_{4}, 3-\mathrm{O}_{2} \mathrm{~N}-4-\mathrm{Cl}_{-} \mathrm{C}_{6} \mathrm{H}_{3}$, Pyrazin-2-yl, 4-HO- $\mathrm{C}_{6} \mathrm{H}_{4}, 3,4-d i-\mathrm{Cl}_{2} \mathrm{C}_{6} \mathrm{H}_{3}, 3,4-\mathrm{di}_{2}-\mathrm{F}-\mathrm{C}_{6} \mathrm{H}_{3}$; $\mathrm{R}^{3}=\mathrm{OC}_{2} \mathrm{H}_{5}, \mathrm{OCH}_{3}, \mathrm{CH}_{3} ; \mathrm{R}^{4}=\mathrm{CH}_{3}, \mathrm{C}_{2} \mathrm{H}_{5} ; \mathrm{Ar}^{1}=\mathrm{C}_{6} \mathrm{H}_{5}, 2-\mathrm{H}_{3} \mathrm{C}-\mathrm{C}_{6} \mathrm{H}_{4}, 3-\mathrm{H}_{3} \mathrm{C}-\mathrm{C}_{6} \mathrm{H}_{4}, 4-\mathrm{H}_{3} \mathrm{C}-\mathrm{C}_{6} \mathrm{H}_{4}, 2-\mathrm{Cl}_{-}-\mathrm{C}_{6} \mathrm{H}_{4}, 3-\mathrm{Cl}-\mathrm{C}_{6} \mathrm{H}_{4}, 4-\mathrm{Cl}-$ $\mathrm{C}_{6} \mathrm{H}_{4}, 2-\mathrm{H}_{3} \mathrm{CO}-\mathrm{C}_{6} \mathrm{H}_{4}, 3-\mathrm{H}_{3} \mathrm{CO}-\mathrm{C}_{6} \mathrm{H}_{4}, 4-\mathrm{H}_{3} \mathrm{CO}-\mathrm{C}_{6} \mathrm{H}_{4} ; \boldsymbol{i}=\mathrm{NH}_{4} \mathrm{OAc} / \mathrm{EtOH} / \mathrm{reflux},{ }^{176} \mathrm{NH}_{4} \mathrm{OAc} / 20 \mathrm{~mol} \% \mathrm{SA} / \mathrm{EtOH} /$ reflux, ${ }^{177} \mathrm{NH}_{4} \mathrm{OAc} / \mathrm{MgO}$ nanotube/ Acetonitrile, reflux, ${ }^{178} \mathrm{NH}_{3}$ dropwise/ $\mathrm{CH}_{3} \mathrm{OH} /$ reflux, ${ }^{179} \mathrm{NH}_{4} \mathrm{OAc} /$ heat $80{ }^{\circ} \mathrm{C} /$ silica $10 \mathrm{~mol} \%,{ }^{180} \mathrm{NH}_{4} \mathrm{OAc} / \mathrm{w}$. b / EtOH, ${ }^{131} \mathrm{NH}_{4} \mathrm{OAc} /$ Bismuth tungstate $\left(\mathrm{Bi}_{2} \mathrm{WO}_{6}\right) 5 \mathrm{~mol} \%,{ }^{181} \mathrm{NH} \mathrm{OAc} \mathrm{OAu} . \mathrm{HCl} /$ $25-30^{\circ} \mathrm{C},{ }^{182} \mathrm{NH}_{4} \mathrm{OAc} /$ Acetonitrile, reflux, $\left.40{ }^{\circ} \mathrm{C}, 300 \mathrm{~W}, 30{ }^{\circ} \mathrm{C}\right) .{ }^{183}$

Scheme 78. Synthesis of 1,4-dihydropyridines 182 and 183.

3.1.5. Monocyclic six-membered with two heteroatoms. 3.1.5.1. Pyrimidine derivatives. Condensation of pyrazol-4-ylmethylene malononitrile $\mathbf{5 4}$ with thiourea $\mathbf{1 8 4}$ in the presence of sodium ethoxide solution at reflux gave the corresponding pyrimidine derivatives $195 .{ }^{165}$ Also, it was reported that the cyclocondensation of pyrazole-carboxaldehyde 2 with urea ${ }^{120}$ or thiourea $184^{120,185}$ and ethyl cyanoacetate 64 in the presence of 
sodium ethoxide ${ }^{120}$ or potassium carbonate ${ }^{185}$ gave the corresponding 2-oxo(thioxo)pyrimidine derivatives 185 (Scheme 79).

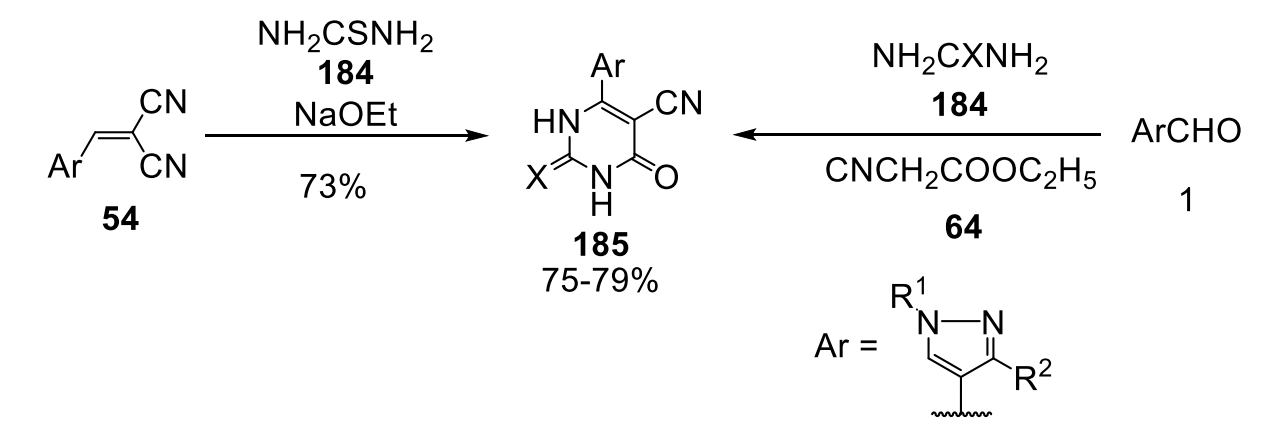

$$
\mathrm{R}^{1}=\mathrm{C}_{6} \mathrm{H}_{5}, 3-\mathrm{Cl}-\mathrm{C}_{6} \mathrm{H}_{4} ; \mathrm{R}^{2}=2-\mathrm{HO}-3,4-\mathrm{di}-\mathrm{H}_{3} \mathrm{CO}-\mathrm{C}_{6} \mathrm{H}_{2}, 4-\mathrm{H}_{3} \mathrm{CO}-\mathrm{C}_{6} \mathrm{H}_{4}, \mathrm{C}_{6} \mathrm{H}_{5} ; \mathrm{X}=\mathrm{O}, \mathrm{S}
$$

Scheme 79. Synthesis of 2-oxo(thioxo)pyrimidine derivatives 185.

The reaction of $\alpha, 6$-unsaturated ketone $\mathbf{4 2}$ with thiourea $\mathbf{1 8 4}$ in the presence of sodium ethoxide solution at reflux was reported to give either the corresponding pyrimidine derivatives $\mathbf{1 8 6 , 1 0 4 , 1 1 4 , 1 1 5 , 1 6 5} \mathbf{1 8 7 ,}{ }^{141,157}$ or $188^{186}$ (Scheme 80).

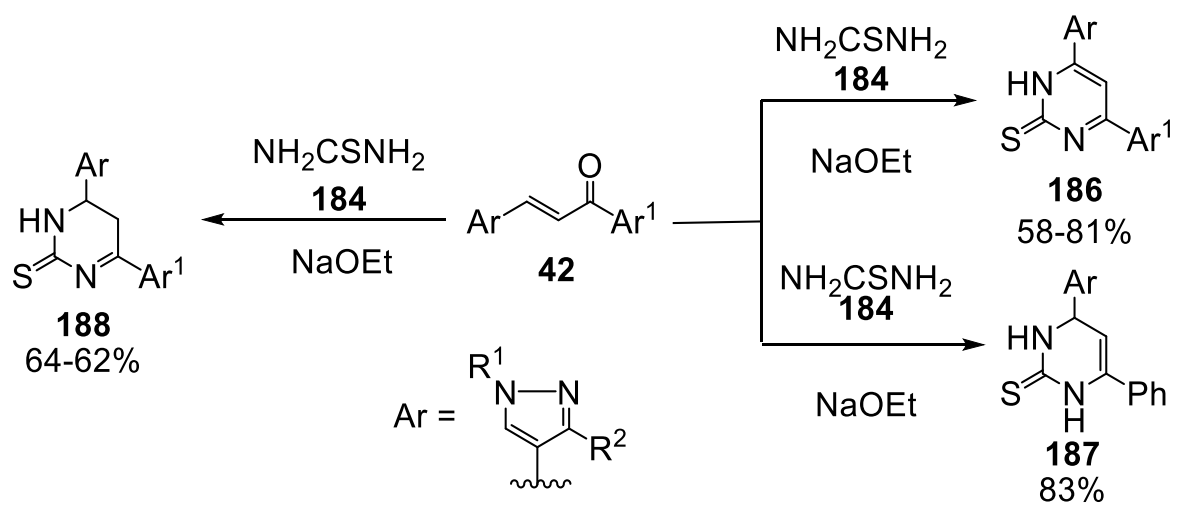

$\mathrm{R}^{1}=\mathrm{C}_{6} \mathrm{H}_{5}, 3-\mathrm{Cl}-\mathrm{C}_{6} \mathrm{H}_{4} ; \mathrm{R}^{2}=2-\mathrm{HO}-3,4-\mathrm{di}-\mathrm{H}_{3} \mathrm{CO}-\mathrm{C}_{6} \mathrm{H}_{2}, 4-\mathrm{H}_{3} \mathrm{CO}-\mathrm{C}_{6} \mathrm{H}_{4}, \mathrm{C}_{6} \mathrm{H}_{5}, 4-\mathrm{H}_{3} \mathrm{C}-\mathrm{C}_{6} \mathrm{H}_{4}, 4-\mathrm{H}_{3} \mathrm{CO}-\mathrm{C}_{6} \mathrm{H}_{4}, 4-\mathrm{HO}_{6}-\mathrm{C}_{6} \mathrm{H}_{4}, 2,4-$ di- ${ }_{2} \mathrm{~N}-\mathrm{C}_{6} \mathrm{H}_{3}, 2,4-\mathrm{di}-\mathrm{H}_{3} \mathrm{CO}-\mathrm{C}_{6} \mathrm{H}_{3}, 2-\mathrm{HO}-\mathrm{C}_{6} \mathrm{H}_{4}, \mathrm{Ar}^{1}=\mathrm{C}_{6} \mathrm{H}_{5}, 2-\mathrm{O}$ xo-2H-chromen-3-yl, 4- $\mathrm{H}_{3} \mathrm{CO}-\mathrm{C}_{6} \mathrm{H}_{4}, 4-\mathrm{H}_{3} \mathrm{C}-\mathrm{C}_{6} \mathrm{H}_{4}, 4-\mathrm{Cl}-$ $\mathrm{C}_{6} \mathrm{H}_{4}, 4-\mathrm{F}-\mathrm{C}_{6} \mathrm{H}_{4}, 4-\mathrm{HO}-\mathrm{C}_{6} \mathrm{H}_{4}, 2-\mathrm{HO}-\mathrm{C}_{6} \mathrm{H}_{4}, 4-\mathrm{O}_{2} \mathrm{~N}-\mathrm{C}_{6} \mathrm{H}_{4}, 4-\mathrm{Br}_{-}-\mathrm{C}_{6} \mathrm{H}_{4}$, 2-Thienyl.

Scheme 80. Synthesis of pyrimidinethione derivatives 186, 187 and 188.

On the other hand treatment of chalcones $\mathbf{4 2}$ with guanidine hydrochloride or guanidine sulfate $\mathbf{1 8 4}$ at reflux afforded pyrimidin-2-amines $\mathbf{1 8 9}^{104,109,114,115}$ (Scheme $\mathbf{8 1 )}$.

Ismail et al. ${ }^{118}$ reported that the reaction of pyrazol-4-ylmethylene malononitrile $\mathbf{5 4}$ with urea or thiourea 184 afforded 4,6-diamino-5-benzylidenepyrimidin-2(5H)-ones/(thiones) 190 (Scheme 82). 


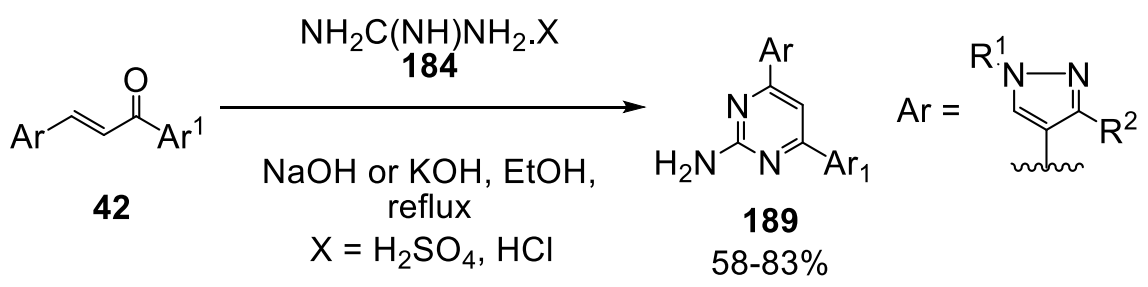

$\mathrm{R}^{1}=\mathrm{C}_{6} \mathrm{H}_{5}, 3-\mathrm{Cl}-\mathrm{C}_{6} \mathrm{H}_{4} ; \mathrm{R}^{2}=\mathrm{C}_{6} \mathrm{H}_{5}, 4-\mathrm{F}-\mathrm{C}_{6} \mathrm{H}_{4}, 4-\mathrm{Cl}-\mathrm{C}_{6} \mathrm{H}_{4}, 3-\mathrm{Br}_{-} \mathrm{C}_{6} \mathrm{H}_{4}, 4-\mathrm{Br}-\mathrm{C}_{6} \mathrm{H}_{4}, 3-\mathrm{O}_{2} \mathrm{~N}-\mathrm{C}_{6} \mathrm{H}_{4}, 4-\mathrm{O}_{2} \mathrm{~N}-\mathrm{C}_{6} \mathrm{H}_{4}, 4-\mathrm{H}_{3} \mathrm{C}-\mathrm{C}_{6} \mathrm{H}_{4}, 4-$ $\mathrm{H}_{3} \mathrm{CO}-\mathrm{C}_{6} \mathrm{H}_{4} ; \mathrm{Ar}^{1}=$ Pyridin-3-yl, Thiophen-2-yl, $\mathrm{C}_{6} \mathrm{H}_{5}, 4-\mathrm{H}_{3} \mathrm{CO}-\mathrm{C}_{6} \mathrm{H}_{4}, 4-\mathrm{H}_{3} \mathrm{C}-\mathrm{C}_{6} \mathrm{H}_{4}, 4-\mathrm{Cl}-\mathrm{C}_{6} \mathrm{H}_{4}, 4-\mathrm{F}-\mathrm{C}_{6} \mathrm{H}_{4}, 4-\mathrm{HO}-\mathrm{C}_{6} \mathrm{H}_{4}, 2-$ $\mathrm{HO}-\mathrm{C}_{6} \mathrm{H}_{4}, 4-\mathrm{O}_{2} \mathrm{~N}-\mathrm{C}_{6} \mathrm{H}_{4}, 4-\mathrm{Br}-\mathrm{C}_{6} \mathrm{H}_{4}$.

Scheme 81. Synthesis of pyrimidin-2-amines 189.

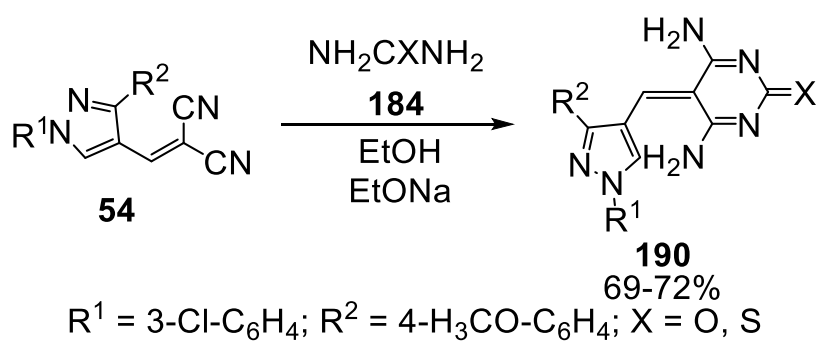

Scheme 82. Synthesis of 4,6-diamino-5-benzylidenepyrimidin-2(5H)-ones/(thiones) 190.

Knovenagel condensation of pyrazole-carboxaldehyde 2 with barbituric acid, thiobarbituric acid 191 afforded the corresponding pyrimidine-2,4,6(1H,3H,5H)-trione and dihydro-2-thioxopyrimidine-4,6(1H,5H)dione $192^{118,126}$ (Scheme 83).

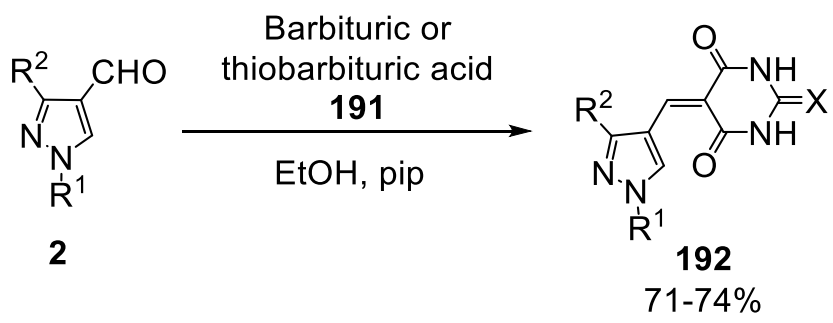

$$
\mathrm{R}^{1}=3-\mathrm{Cl}-\mathrm{C}_{6} \mathrm{H}_{4}, \mathrm{C}_{6} \mathrm{H}_{5} ; \mathrm{R}^{2}=4-\mathrm{H}_{3} \mathrm{CO}-\mathrm{C}_{6} \mathrm{H}_{4}, 4-\left(\mathrm{H}_{5} \mathrm{C}_{6} \mathrm{H}_{2} \mathrm{CO}\right)-\mathrm{C}_{6} \mathrm{H}_{4} ; \mathrm{X}=\mathrm{O}, \mathrm{S}
$$

Scheme 83. Synthesis of dihydro-2-thioxopyrimidine-4,6(1H,5H)-dione 192.

Under various conditions multi-component reaction of pyrazole-4-carboxaldehyde 2 with ethylacetoacetate, ${ }^{120,131,187-189}$ methylacetoacetate, ${ }^{131,187}$ acetylacetone ${ }^{120,131}$ or butanamides ${ }^{64,190,191}$ 64 and urea $^{64,120,131,187,189-191}$ thiourea, ${ }^{64,103,120,187,188,191}$ guanidine $^{187}$ or 1-methylurea ${ }^{189} \mathbf{1 8 4}$ afforded the corresponding 2-oxo (thioxo) pyrazole substituted pyrimidine derivatives 193 (Scheme 84).

TEAA = triethylammonium acetate; $p$-TsOH $=p$-toluenesulfonic acid 


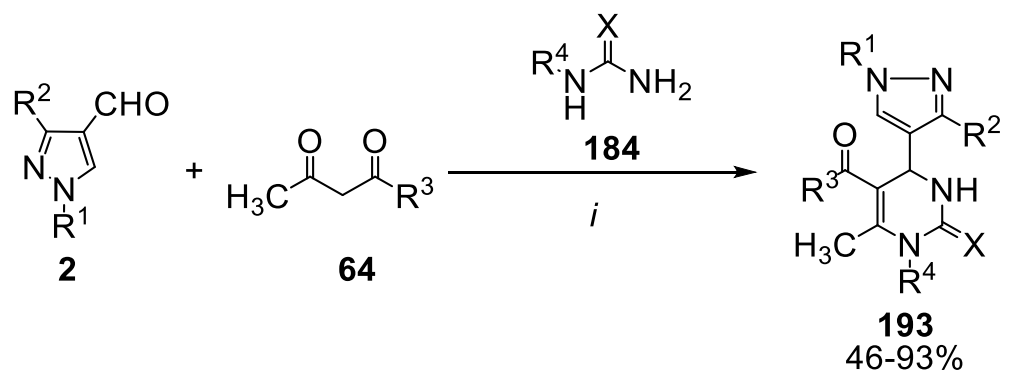

$\mathrm{R}^{1}=\mathrm{C}_{6} \mathrm{H}_{5}, 3-\mathrm{Cl}_{-} \mathrm{C}_{6} \mathrm{H}_{4}, 4-\mathrm{F}-\mathrm{C}_{6} \mathrm{H}_{4}, 2,4-$ di- $\mathrm{F}-\mathrm{C}_{6} \mathrm{H}_{3} ; \mathrm{R}^{2}=3,4-\mathrm{di}-\mathrm{Cl}_{-} \mathrm{C}_{6} \mathrm{H}_{3}, 3,4$ di-F- $\mathrm{C}_{6} \mathrm{H}_{3}, 4-\mathrm{H}_{3} \mathrm{CO}-\mathrm{C}_{6} \mathrm{H}_{4}, 4-\mathrm{F}-\mathrm{C}_{6} \mathrm{H}_{4}, 4-\mathrm{Cl}_{-}-\mathrm{C}_{6} \mathrm{H}_{4}$, 4- $\mathrm{Br}-\mathrm{C}_{6} \mathrm{H}_{4}, 4-\mathrm{O}_{2} \mathrm{~N}-\mathrm{C}_{6} \mathrm{H}_{4}, 4-\mathrm{H}_{3} \mathrm{C}-\mathrm{C}_{6} \mathrm{H}_{4}, \mathrm{C}_{6} \mathrm{H}_{5}, 2,4-\mathrm{di}-\mathrm{Cl}-5-\mathrm{F}-\mathrm{C}_{6} \mathrm{H}_{2}, 3-\mathrm{Br}_{-} \mathrm{C}_{6} \mathrm{H}_{4}, 3-\mathrm{O}_{2} \mathrm{~N}-\mathrm{C}_{6} \mathrm{H}_{4}, \mathrm{CH}_{3}, \mathrm{C}_{2} \mathrm{H}_{5}, 4-\mathrm{HO}-\mathrm{C}_{6} \mathrm{H}_{4}, 2-\mathrm{HO}-$ $\mathrm{C}_{6} \mathrm{H}_{4} ; \mathrm{R}^{3}=\mathrm{OC}_{2} \mathrm{H}_{5}, \mathrm{OCH}_{3}, \mathrm{CH}_{3}$, Pyridin-2-yl- $\mathrm{NH},\left(5-\mathrm{H}_{3} \mathrm{C}\right.$-pyridin-2-yl)- $\mathrm{NH},\left(5-\mathrm{Br}\right.$-pyridin-2-yl)- $\mathrm{NH}, \mathrm{C}_{6} \mathrm{H}_{5} \mathrm{NH}, \mathrm{NH}-\left(4-\mathrm{H}_{3} \mathrm{C}-\right.$ $\left.\mathrm{C}_{6} \mathrm{H}_{4}\right), \mathrm{NH}-\left(4-\mathrm{H}_{3} \mathrm{CO}-\mathrm{C}_{6} \mathrm{H}_{4}\right), \mathrm{NH}-\left(4-\mathrm{Cl}-\mathrm{C}_{6} \mathrm{H}_{4}\right), \mathrm{NH}-\left(2-\mathrm{Cl}_{-} \mathrm{C}_{6} \mathrm{H}_{4}\right), \mathrm{NH}-\left(4-\mathrm{O}_{2} \mathrm{~N}-\mathrm{C}_{6} \mathrm{H}_{4}\right), \mathrm{NH}-\left(2-\mathrm{F}-\mathrm{C}_{6} \mathrm{H}_{4}\right), \mathrm{NH}-\left(3-\mathrm{F}-\mathrm{C}_{6} \mathrm{H}_{4}\right), \mathrm{NH}-$ $\left(3-\mathrm{Cl}-\mathrm{C}_{6} \mathrm{H}_{4}\right), \mathrm{NH}-\left(2-\mathrm{O}_{2} \mathrm{~N}-\mathrm{C}_{6} \mathrm{H}_{4}\right), \mathrm{NH}-\left(2-\mathrm{F}_{-} \mathrm{C}_{6} \mathrm{H}_{4}\right), \mathrm{NH}-\left(3-\mathrm{O}_{2} \mathrm{~N}-\mathrm{C}_{6} \mathrm{H}_{4}\right) ; \mathrm{R}^{4}=\mathrm{H}, \mathrm{CH}_{3} ; \mathrm{X}=\mathrm{S}, \mathrm{O}, \mathrm{NH} . \quad i=\mathrm{HCl} /$ EtOH, ${ }^{103,120,131,187,188}$ TEAA, ${ }^{190} p$-TsOH 40 mol \%/ EtOH, ${ }^{64} \mathrm{CH}_{3} \mathrm{OH} / \mathrm{HCl}^{191} \mathrm{FeCl}_{3} \cdot 6 \mathrm{H}_{2} \mathrm{O},{ }^{192}$ Phosphotungstic acid. ${ }^{189}$

Scheme 84. Synthesis of 2-oxo (thioxo) pyrazole substituted pyrimidine derivatives 193.

On the other hand condensation of 1-(3-chlorophenyl)-3-(4-methoxyphenyl)-1H-pyrazole-4carboxaldehyde $\mathbf{2}$ with ethyl cyanoacetate or ethyl acetoacetate 64 in the presence of guanidine hydrochloride 184 gave 2-amino-5-cyano/acetyl-6-hydroxy-4-aryl pyrimidines $194^{104}$ (Scheme 85).

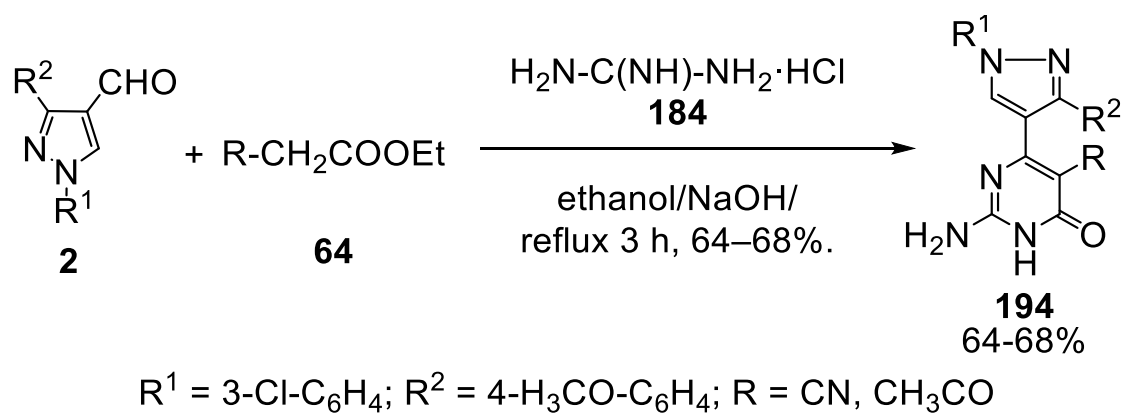

Scheme 85. Synthesis of 2-amino-5-cyano/acetyl-6-hydroxy-4-aryl pyrimidines 194.

3.1.6. Monocyclic six-membered with three heteroatoms. 3.1.6.1. Triazine derivatives. Heating a solution of thiosemicarbazide derivative 67 in acetic acid and $\mathrm{HCl}$ at reflux produced 5-((1,3-diphenyl-1H-pyrazole-4yl)methyl)-3-(phenylamino)-1,2,4-triazin-6(1H)-one $195^{123}$ (Scheme 86).

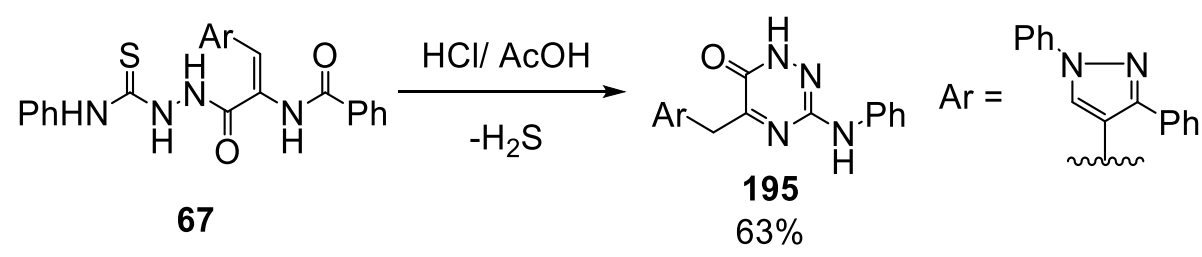

Scheme 86. Synthesis of 5-((1H-pyrazole-4-yl)methyl)-3-(phenylamino)-1,2,4-triazin-6(1H)-one 195.

3.1.7. Monocyclic six-membered with four heteroatoms. 3.1.7.1. Tetrazine derivatives. El-Bordany et al. ${ }^{145}$ reported that the reaction of pyrazolyl thiocarbohydrazone derivative $\mathbf{1 0 0}$ with 4-chlorobenzaldehyde $\mathbf{4 3}$ in 
ethanol at reflux gave instead of a condensation product, the cyclized adduct pyrazolyl-tetrazinethione derivative 196. Reaction of 100 with hydrazine hydrate 46 in ethanol at reflux afforded corresponding 1,2,4,5tetrazine derivative 197. Compound 197 was alternatively obtained by reaction of 196 with hydrazine hydrate 46 in ethanol at reflux (Scheme 87).

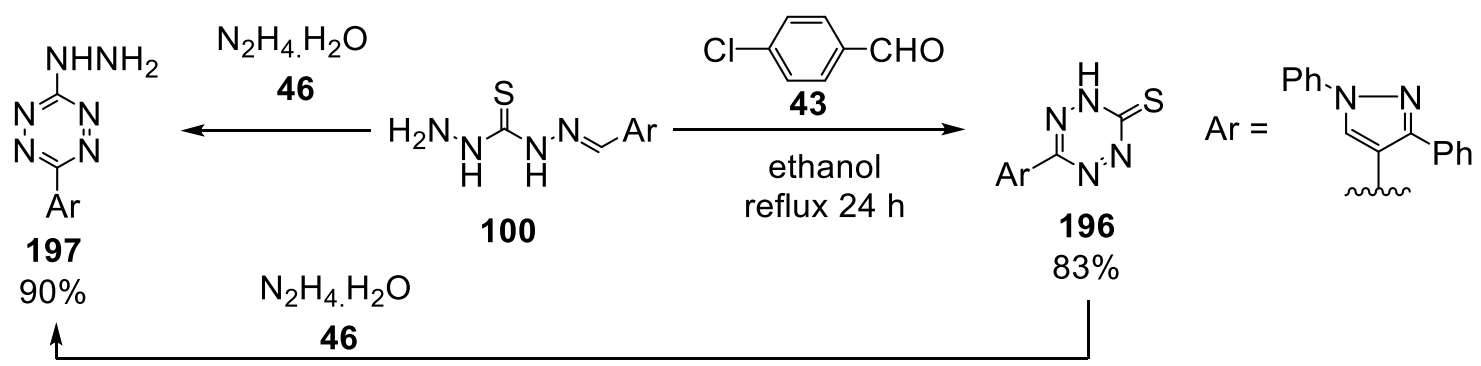

Scheme 87. Synthesis of pyrazolyl-tetrazinethione derivatives 196 and 197.

3.1.7.2. Oxadiazaphosphinin derivatives. Ali ${ }^{117}$ reported that heating of 2-cyano- $N$-[1,3-diphenyl-1H-pyrazol-4ylmethylidene]acetohydrazide 58 with diethyl phosphite 125 and boron trifluoride etherate afforded [2-ethoxy2-oxido-3-(1,3-diphenyl-1H-pyrazol-4-yl)-2H-1,4,5,2-oxadiazaphosphinin-6-yl]acetonitrile 198 in good yield (Scheme 88).

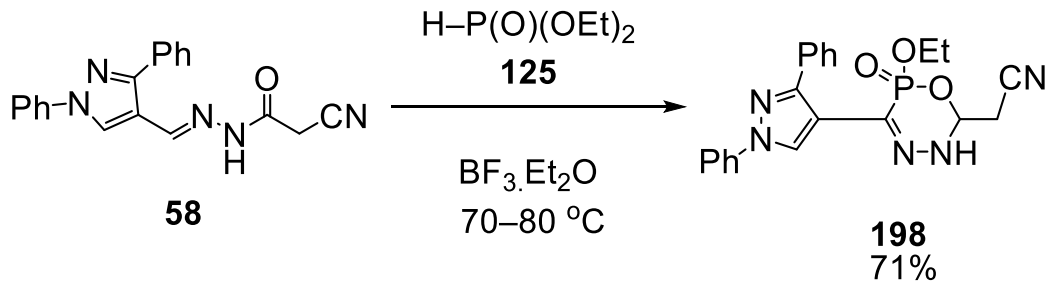

Scheme 88. Synthesis of [3-(1H-pyrazol-4-yl)-2H-1,4,5,2-oxadiazaphosphinin-6-yl]acetonitrile 198.

\subsection{Pyrazole-substituted fused heterocyclic system}

3.2.1. Pyrazole-substituted bicyclic systems. 3.2.1.1. Fused [5-6] system with two heteroatoms. 3.2.1.1.1. Thiazolo[3,2-a]pyridine derivative. El-Emary et al. ${ }^{166}$ reported that condensation of 2-cyanomethyl-4thiazolinone 199 with 1,3-diphenyl-pyrazole-4-carboxaldehyde 2 yielded 2-(5-((1,3-diphenyl-1H-pyrazol-4$\mathrm{yl}$ )methylene)-4-oxo-4,5-dihydrothiazol-2-yl)acetonitrile $\mathbf{2 0 0}$. Heating of $\mathbf{2 0 0}$ with malononitrile in ethanol at reflux gave the corresponding thiazolo[3,2-a]pyridine derivative 201. On the other hand, treatment of compound 201 with arylidenemalononitriles 172 gave the thiazolo[3,2-a]pyridine derivatives 202. Thiazolo[3,2a]pyridines $\mathbf{2 0 2}$ were also synthesized via a multi-component reaction of compound $\mathbf{2 0 0}$ with aromatic aldehyde $\mathbf{4 3}$ and malononitrile $\mathbf{5 3}$ in ethanol containing piperidine at reflux (Scheme 89). 


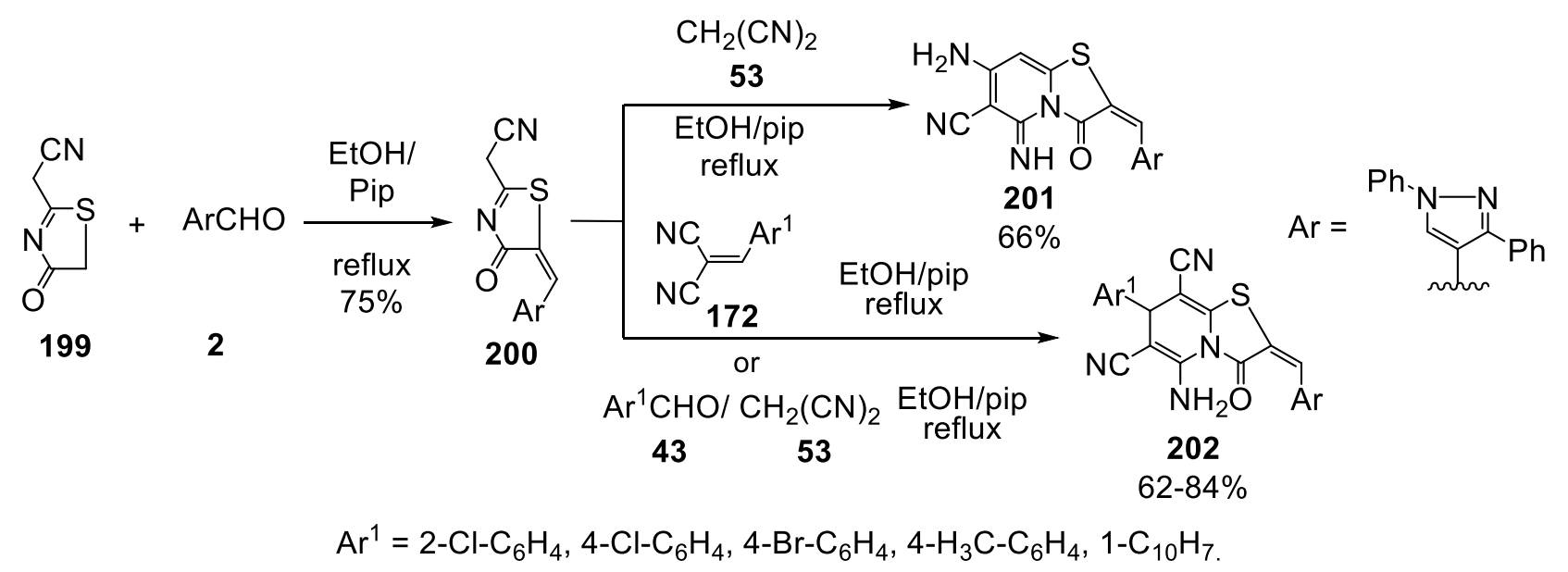

Scheme 89. Synthesis of thiazolo[3,2-a]pyridine derivatives 201 and 202.

Abdel Hafiz et al. ${ }^{162}$ reported that the formation of 5-amino-3-oxo-2,3,6,7-tetrahydro-5 H-thiazolo[3,2a]pyridine-6-carbonitrile $\mathbf{2 0 3}$ was performed by the reaction of pyrazol-4-ylmethylene malononitrile $\mathbf{5 4}$ with thiazol-4(5H)-one derivatives 199 (Scheme 90).

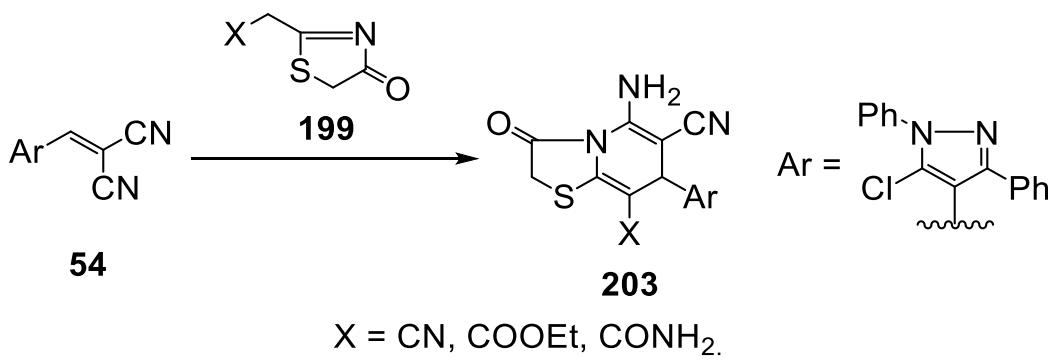

Scheme 90. Synthesis of 5-amino-3-oxo-tetrahydro-5H-thiazolo[3,2-a]pyridine-6-carbonitrile 203.

3.2.1.1.2. Fused [5-6] system with three heteroatoms. 3.2.1.1.2.1. Pyrazolo[3,4-b]pyridine derivatives. Jachak et al. ${ }^{193}$ reported that pyrazolo[3,4-b]pyridine derivatives 205 were synthesized by one-pot cyclocondensation of 5-amino-3-aryl-1H-phenylpyrazoles 204, $p$-substituted benzoylacetonitriles 101, and pyrazole-4carboxaldehydes $\mathbf{2}$ using ammonium acetate or triethylamine as a catalyst (Scheme 91).

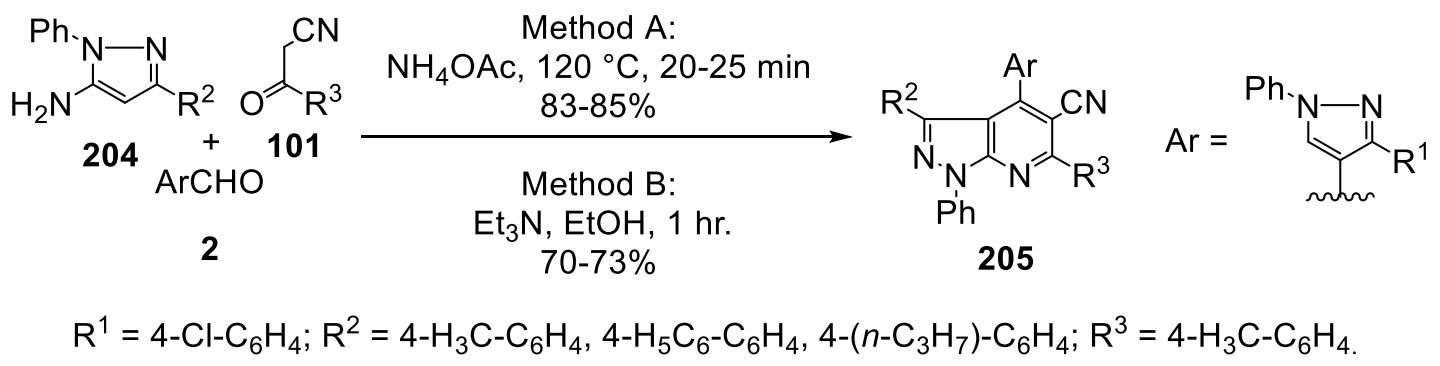

Scheme 91. Synthesis of pyrazolo[3,4-b]pyridine derivatives 205.

3.2.1.1.2. Thiazolo[3,2-a]pyrimidine derivatives. Sahu et al. ${ }^{194}$ reported the synthesis of a series thiazolo[3,2a]pyrimidine-6-carboxylate derivatives 207 through a multi-component reaction of ethyl acetoacetate 64, 
pyrazole-4-carboxaldehydes 2, and an excess amount of substituted aminothiazole 206 using ammonium metavanadate $\left(\mathrm{NH}_{4} \mathrm{VO}_{3}\right)$ as a catalyst (Scheme 92$)$.

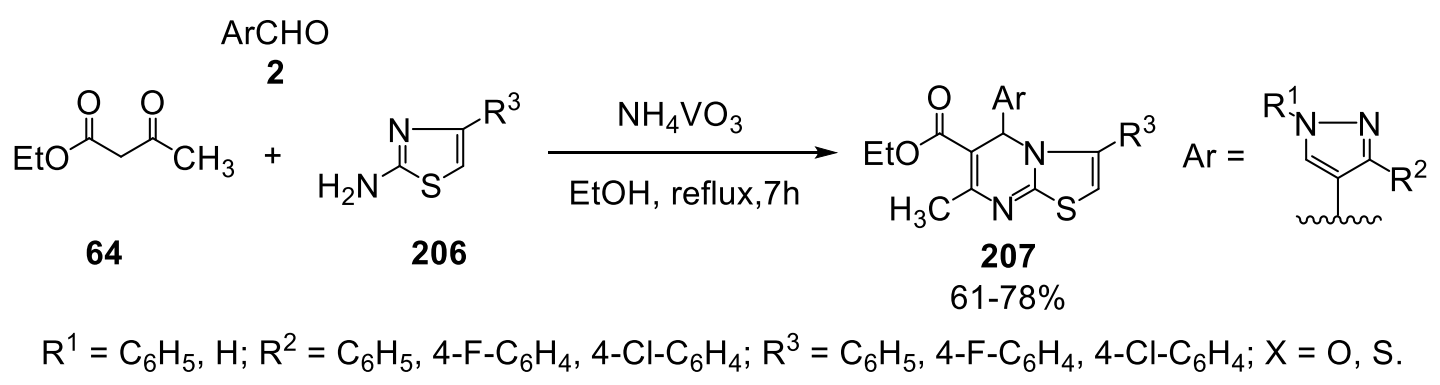

Scheme 92. Synthesis of thiazolo[3,2-a]pyrimidine-6-carboxylate derivatives 207.

3.2.1.1.2.3. $1 H$-Imidazo[4,5-b]pyridine derivatives. Kumbar et al. ${ }^{195}$ reported the synthesis of 6 -bromo- $1 H$ imidazo[4,5-b]pyridines 209 by condensation of 6-bromo-pyridine-2,3-diamine 208 with pyrazole-4carboxaldehydes $\mathbf{2}$ in ethanol at reflux or under microwave irradiation (Scheme 93).

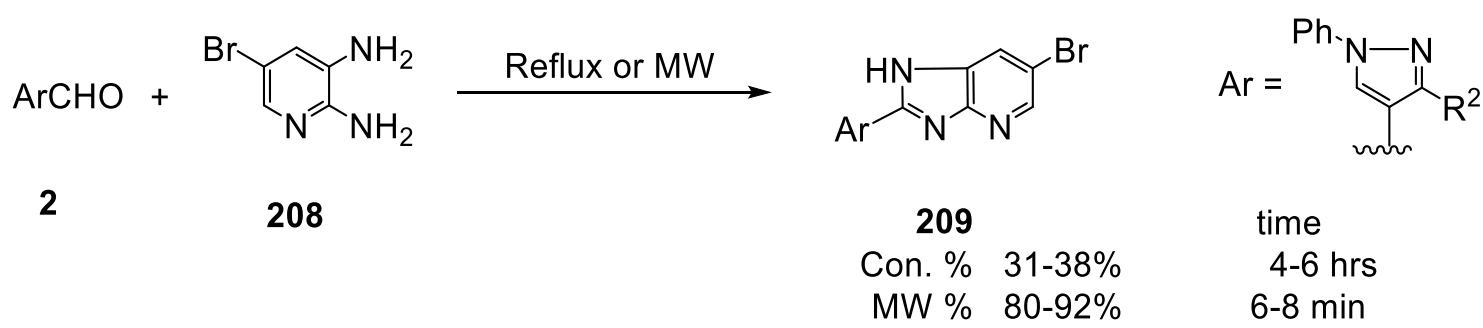

$\mathrm{R}^{2}=$ 2-Oxo-3,8a-dihydro-2H-chromen-3-yl, 6-Cl-2-oxo-3,8a-dihydro-2H-chromen-3-yl, 6-Br-2-oxo-3,8a-dihydro$2 \mathrm{H}$-chromen-3-yl, 8- $\mathrm{H}_{3} \mathrm{CO}-2-0 x \mathrm{o}-3,8 \mathrm{a}-$ dihydro-2H-chromen-3-yl.

Scheme 93. Synthesis of 6-bromo-1H-imidazo[4,5-b]pyridines 209.

3.2.1.1.3. Fused [5-6] system with four heteroatoms. 3.2.1.1.3.1. [1,2,4]Triazolo[4,3-a]pyrimidine derivatives. Kamal et al. ${ }^{196}$ reported that hydrazone derivatives 211 were obtained by heating the 2- hydrazino-4,6dimethylpyrimidine $\mathbf{2 1 0}$ with the appropriate pyrazole-4-carboxaldehyde derivatives $\mathbf{2}$ in ethanol at reflux. [1,2,4]Triazolo[4,3-a]-pyrimidines $\mathbf{2 1 2}$ were obtained by oxidation of hydrazone derivatives 211 using iodobenzebediacetate (IBD) in dichloromethane (DCM) at room temperature (Scheme 94).<smiles>CCC(C=O)C(C)O</smiles>

210<smiles>Cc1cc(C)nc(NC=[Al]CCl)n1</smiles>

211

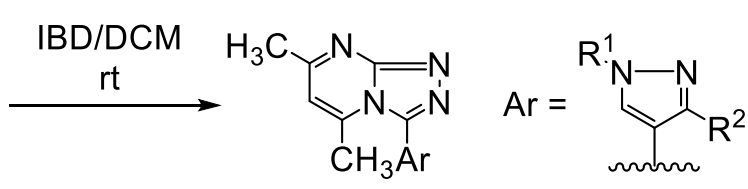

212

$86-96 \%$

$\mathrm{R}^{1}=\mathrm{C}_{6} \mathrm{H}_{5} ; \mathrm{R}^{2}=\mathrm{C}_{6} \mathrm{H}_{5}, 4-\mathrm{H}_{3} \mathrm{C}-\mathrm{C}_{6} \mathrm{H}_{4}, 4-\mathrm{H}_{3} \mathrm{CO}-\mathrm{C}_{6} \mathrm{H}_{4}, 2-\mathrm{H}_{3} \mathrm{CO}-\mathrm{C}_{6} \mathrm{H}_{4}, 4-\mathrm{O}_{2} \mathrm{~N}-\mathrm{C}_{6} \mathrm{H}_{4}, 3-\mathrm{O}_{2} \mathrm{~N}-\mathrm{C}_{6} \mathrm{H}_{4}, 4-\mathrm{Cl}-\mathrm{C}_{6} \mathrm{H}_{4}, 4-\mathrm{Br}-\mathrm{C}_{6} \mathrm{H}_{4}, 4-\mathrm{F}-$ $\mathrm{C}_{6} \mathrm{H}_{4}, 2-\mathrm{Naphthyl}$.

Scheme 94. Synthesis of [1,2,4]triazolo[4,3-a]-pyrimidines 212. 
3.2.1.1.3.2. Triazolo[1,5-a]pyrimidine derivatives. Shejale et al. ${ }^{157}$ and El-Emary and Bakhite ${ }^{141}$ reported that the reaction of chalcones $\mathbf{4 2}$ with 3-amino-s-triazole 213 in acetic acid at reflux afforded 4,7-dihydro-7-\{1phenyl-3-(substituted phenyl)-1H-pyrazol-4-yl\}-5-phenyl-s-triazolo[1,5-a]pyrimidine 214 (Scheme 95).

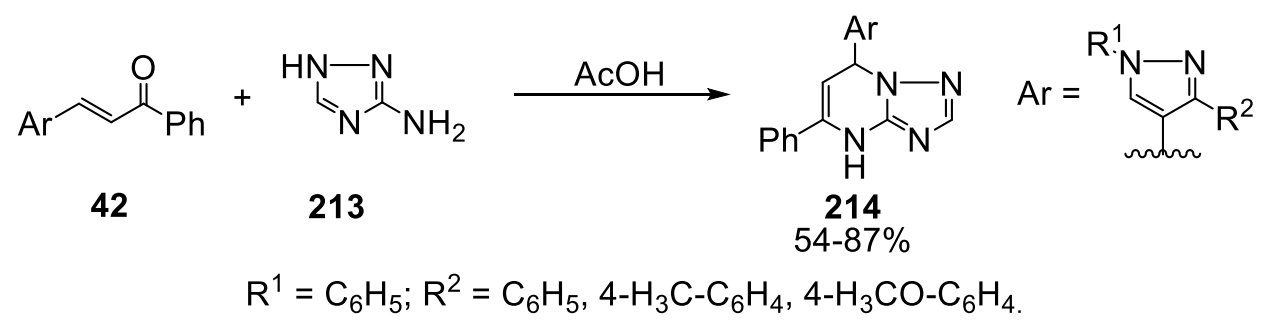

Scheme 95. Synthesis of 7-\{1H-pyrazol-4-yl\}-5-phenyl-s-triazolo[1,5-a]pyrimidines 214.

Bhatt et al. ${ }^{197}$ reported the synthesis of 4,7-dihydro-[1,2,4]-triazolo[1,5-a]pyrimidine-6-carboxamides 215 via Biginelli reaction of 1-phenyl-3-aryl-1H-pyrazol-4-carboxaldehyde 2, $1 \mathrm{H}$-1,2,4-triazol-3-amine 213 and acetoacetanilide derivatives 64 under conventional and microwave irradiation conditions (Scheme 96).

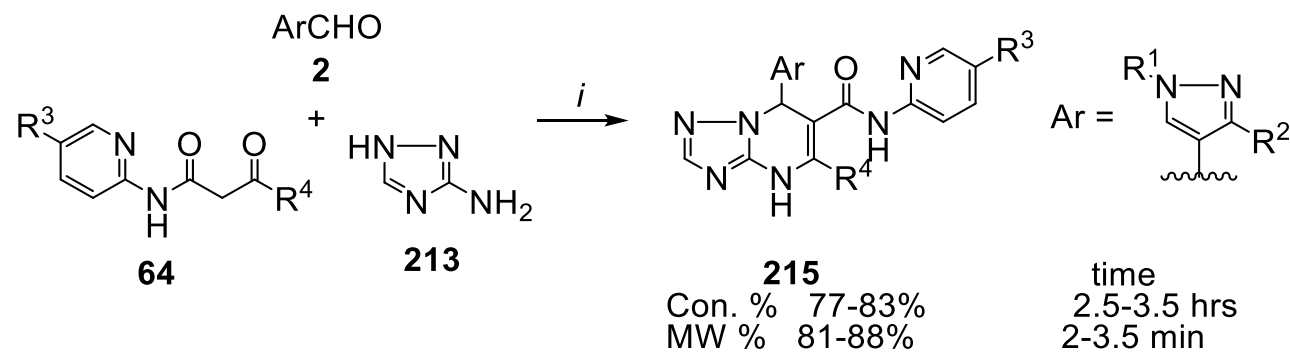

$\boldsymbol{i}=$ Conventional heating or MW irradiation/ solvent of reaction in the both conditions [DMF, EtOH, $(\mathrm{EtOH}$, $\mathrm{HCl}$ ), (EtOH, PTSA), TEAA]; $\mathrm{R}^{1}=\mathrm{C}_{6} \mathrm{H}_{5} ; \mathrm{R}^{2}=\mathrm{C}_{6} \mathrm{H}_{5}, 4-\mathrm{H}_{3} \mathrm{C}-\mathrm{C}_{6} \mathrm{H}_{4}, 4-\mathrm{O}_{2} \mathrm{~N}-\mathrm{C}_{6} \mathrm{H}_{4}, 4-\mathrm{Cl}-\mathrm{C}_{6} \mathrm{H}_{4}, 4-\mathrm{F}-\mathrm{C}_{6} \mathrm{H}_{4} ; \mathrm{R}^{3}=\mathrm{H}, \mathrm{CH}_{3}, \mathrm{Br}^{2} \mathrm{R}^{4}$ $=-\mathrm{CH}_{3},-\mathrm{HC}\left(\mathrm{CH}_{3}\right)_{2}$.

Scheme 96. Synthesis of 4,7-dihydro-[1,2,4]-triazolo[1,5-a]pyrimidine-6-carboxamides 215.

3.2.1.3.3. Pyrazolo[3,4-d]pyrimidine derivatives. The reaction of 5-methyl-2-phenyl-2,4-dihydro-3H-pyrazol3-one 111 with pyrazol-4-carboxaldehyde $\mathbf{2}$ in the presence of sodium acetate and PEG-400 as a solvent gave the corresponding 4-(arylidene)-3-methyl-1-phenyl-1H-pyrazol-5-ones $\mathbf{1 1 2}$. The reaction of 112 with guanidine hydrochloride 184 and sodium hydroxide in the presence of PEG-400 afforded the corresponding pyrazolo[3,4d] pyrimidin-6-amine derivatives $216^{198}$ (Scheme 97).

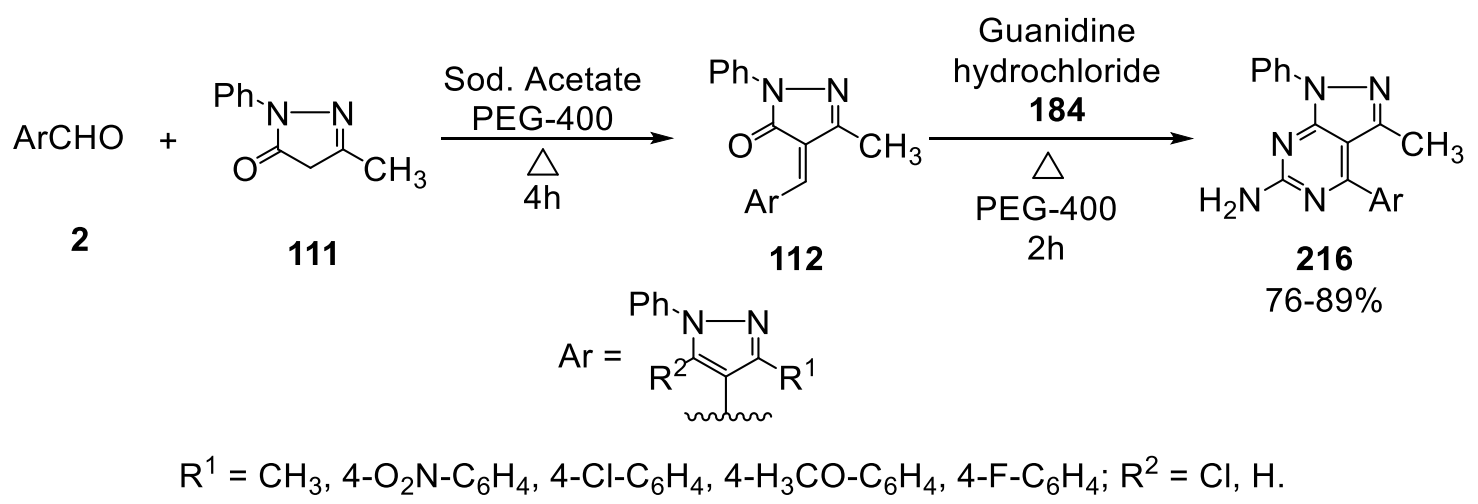

Scheme 97. Synthesis of pyrazolo[3,4-d]pyrimidin-6-amine derivatives 216. 
3.2.1.1.4. Fused [5-6] system with five heteroatoms. 3.2.1.1.4.1. 1,2,4-Triazolo[4,3-b]1,2,4-triazine derivatives. Hamama et al. ${ }^{199}$ reported that the condensation of 4-amino-6-benzyl-3-hydrazineyl-1,2,4-triazin5(4H)-one 217 with 1,3-diphenyl-1H-pyrazole-4-carboxaldehyde 2 afforded the corresponding Schiff bases 218. Treatment of compound $\mathbf{2 1 8}$ with acetic anhydride gave $\mathrm{N}$-acetyl- $\mathrm{N}$-(2-acetyl-6-benzyl-3-(1,3-diphenyl-1Hpyrazol-4-yl)-7-oxo-2,3-dihydro-1,2,4-triazolo[4,3-b] 1,2,4-triazin-8(7H)-yl)acetamide 219 (Scheme 98).

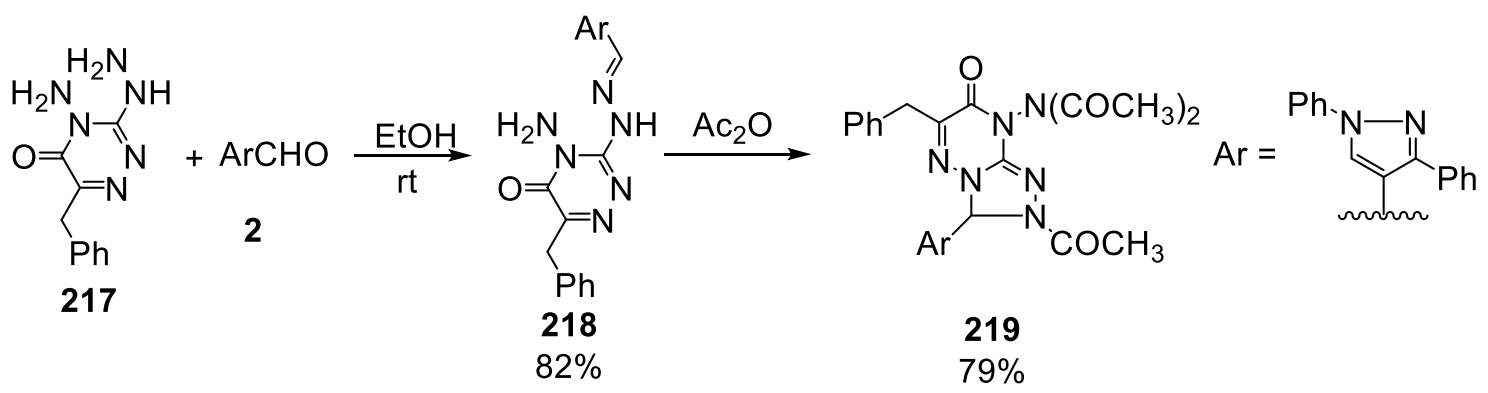

Scheme 98. Synthesis of 3-(1H-pyrazol-4-yl)-1,2,4-triazolo[4,3-b]-1,2,4-triazin-8(7H)-yl)acetamide 219.

3.2.1.1.5. Fused [6-5] system with two heteroatoms. 3.2.1.1.5.1. Benzo[d]imidazole derivatives. Condensation of 0 -phenylenediamines derivatives 68 with pyrazole-4-carboxaldehydes 2 afforded the corresponding 2(pyrazol-4-yl) benzo[d]imidazoles 220195,200-204 (Scheme 99).

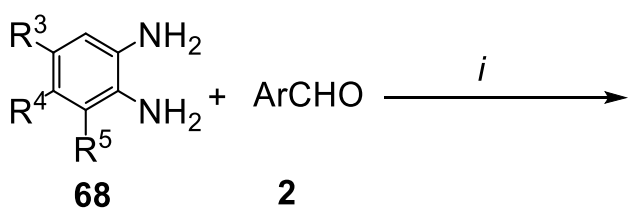

68<smiles>[R]c1cc2nc([Al])[nH]c2c([R])c1[R]</smiles>

220

$30-94 \%$

$\mathrm{R}^{1}=\mathrm{C}_{6} \mathrm{H}_{5}$, Pyridin-2-yl, 4- $\mathrm{H}_{2} \mathrm{NO}_{2} \mathrm{~S}-\mathrm{C}_{6} \mathrm{H}_{4} ; \mathrm{R}^{2}=\mathrm{C}_{6} \mathrm{H}_{5}, 4-\mathrm{O}_{2} \mathrm{~N}-\mathrm{C}_{6} \mathrm{H}_{4}, 4-\mathrm{H}_{3} \mathrm{CO}-\mathrm{C}_{6} \mathrm{H}_{4}, 4-\mathrm{Cl}-\mathrm{C}_{6} \mathrm{H}_{4}, 4-\mathrm{HO}-\mathrm{C}_{6} \mathrm{H}_{4}, 4-\mathrm{H}_{2} \mathrm{~N}-\mathrm{C}_{6} \mathrm{H}_{4}, 4-$ $\mathrm{H}_{3} \mathrm{C}-\mathrm{C}_{6} \mathrm{H}_{4}, 4-\mathrm{Br}_{-} \mathrm{C}_{6} \mathrm{H}_{4}, 4-\mathrm{F}-\mathrm{C}_{6} \mathrm{H}_{4}, 3,4,5-$ tri- $\mathrm{H}_{3} \mathrm{CO}-\mathrm{C}_{6} \mathrm{H}_{2}, 2-\mathrm{HO}-\mathrm{C}_{6} \mathrm{H}_{4}$, 2-oxo-3,8a-dihydro-2H-chromen-3-yl, 6-Cl-2-oxo3,8a-dihydro-2H-chromen-3-yl, $\quad$ 6-Br-2-oxo-3,8a-dihydro- $2 \mathrm{H}$-chromen-3-yl, $\quad$ 8- $\mathrm{H}_{3} \mathrm{CO}-2-0 x \mathrm{o}-3,8 \mathrm{a}-d i h y d r o-2 \mathrm{H}$ chromen-3-yl; $\mathrm{R}^{3}=\mathrm{CH}_{3}, \mathrm{H}, \mathrm{Cl}, \mathrm{NO}_{2}, \mathrm{~F}, \mathrm{Br}, \mathrm{OCH}_{3}, \mathrm{CF}_{3} ; \mathrm{R}^{4}=\mathrm{CH}_{3}, \mathrm{H}, \mathrm{Cl}, \mathrm{NO}_{2} ; \mathrm{R}^{5}=\mathrm{CH}_{3}, \mathrm{H}$. $\boldsymbol{i}=\mathrm{ZnCl}_{2} / \mathrm{CH}_{3} \mathrm{CN}^{200}$, visible light/ Rose Bengal (2mol\%)/ $\mathrm{CH}_{3} \mathrm{CN},{ }^{201} \mathrm{EtOH} / \mathrm{PCl}_{3} / 60{ }^{\circ} \mathrm{C},{ }^{202} \mathrm{Oxone} / \mathrm{DMF} / \mathrm{H}_{2} \mathrm{O},{ }^{203} \mathrm{EtOH} / \mathrm{Na}_{2} \mathrm{~S}_{2} \mathrm{O}_{5} / 50{ }^{\circ} \mathrm{C}-60^{\circ} \mathrm{C}, 204$ reflux or MW. ${ }^{195}$

Scheme 99. Synthesis of 2-(pyrazol-4-yl) benzo[d]imidazoles 220.

3.2.1.1.5.2. Benzo[ $[d]$ oxazole derivatives. Condensation of pyrazole-4-carboxaldehydes 2 with 2 -aminophenol derivatives $\mathbf{2 2 1}$ under different conditions led to the formation of the corresponding benzo[d]oxazole

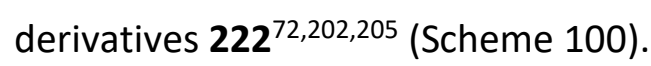

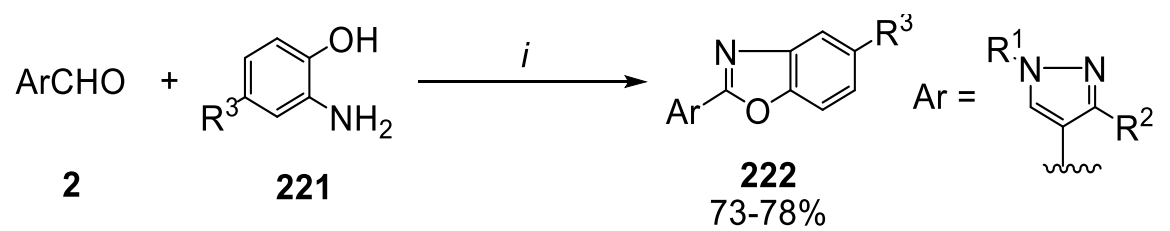

$\mathrm{R}^{1}=\mathrm{C}_{6} \mathrm{H}_{5}$, Pyridin-2-yl; $\mathrm{R}^{2}=2-\mathrm{HO}-\mathrm{C}_{6} \mathrm{H}_{4}, 4-\mathrm{Cl}-\mathrm{C}_{6} \mathrm{H}_{4}, 4-\mathrm{F}_{-} \mathrm{C}_{6} \mathrm{H}_{4}, 4-\mathrm{O}_{2} \mathrm{~N}-\mathrm{C}_{6} \mathrm{H}_{4}, 4-\mathrm{H}_{3} \mathrm{CO}-\mathrm{C}_{6} \mathrm{H}_{4}, 4-\mathrm{H}_{3} \mathrm{C}-\mathrm{C}_{6} \mathrm{H}_{4} ; \mathrm{R}^{3}=\mathrm{H}, \mathrm{Cl} ; \boldsymbol{i}=$ EtOH/ Phosphorus trichloride $\left(\mathrm{PCl}_{3}\right) / 60{ }^{\circ} \mathrm{C},{ }^{202} \mathrm{NH}_{4} \mathrm{Cl}: 70$ mol\% O $\mathrm{O}_{2}$ Air/ $\mathrm{CH}_{3} \mathrm{OH}: \mathrm{H}_{2} \mathrm{O}(15: 1, \mathrm{~V}: \mathrm{V}) \mathrm{rt},{ }^{205}(1) \mathrm{CH}_{3} \mathrm{OH}(2)$ $\mathrm{Phl}(\mathrm{OAc})_{2 .}{ }^{72}$

Scheme 100. Synthesis of benzo[d] oxazole derivatives 222 . 
3.2.1.1.5.3. Benzo[d]thiazole derivatives. The reaction of pyrazole-4-carboxaldehyde 2 with 2aminobenzenethiol $\mathbf{2 2 3}$ in the presence of $\mathrm{PCl}_{3}$ in ethanol afforded the corresponding benzo[d]thiazole $224^{202}$ (Scheme 101).

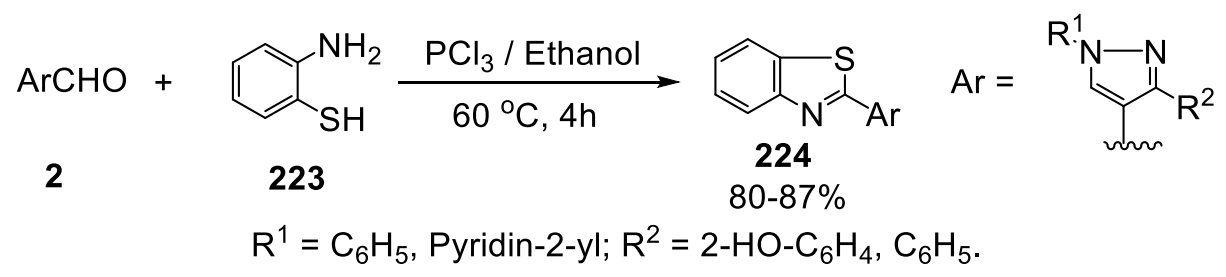

Scheme 101. Synthesis of benzo[d]thiazoles 224.

\subsection{Fused [6-5] system with three heteroatoms. 3.2.1.1.6.1. Pyrano[2,3-c]pyrazole derivatives.} Pyrano[2,3-c]pyrazole derivatives $\mathbf{2 2 5}$ were prepared via the one-pot cyclocondensation reaction of pyrazolone 111, substituted pyrazole-4-carboxaldehydes 2 and malononitrile 53 in polyethylene glycol (PEG-400) as a green solvent ${ }^{206,207}$ or in ethanol at reflux in the presence of piperidine 208 (Scheme 102).

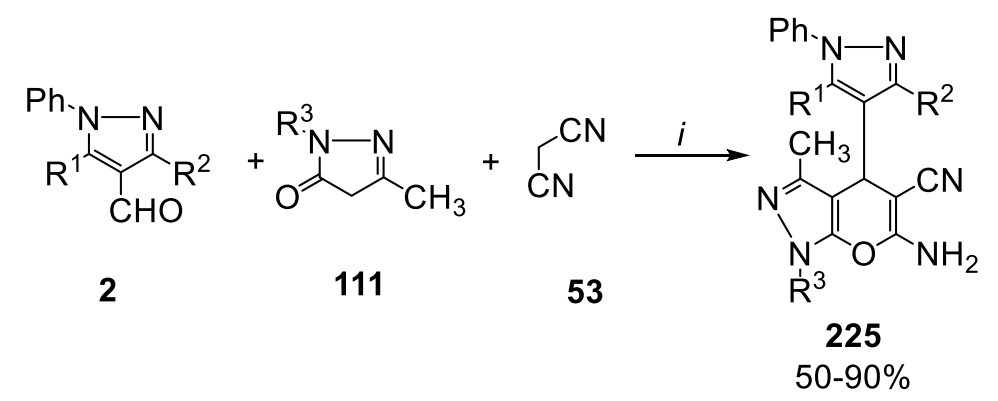

$\mathrm{R}^{1}=\mathrm{H}, \mathrm{Cl} ; \mathrm{R}^{2}=\mathrm{C}_{6} \mathrm{H}_{5}, 4-\mathrm{Br}-\mathrm{C}_{6} \mathrm{H}_{4}, 4-\mathrm{H}_{3} \mathrm{C}-\mathrm{C}_{6} \mathrm{H}_{4}, 4-\mathrm{H}_{3} \mathrm{CO}-\mathrm{C}_{6} \mathrm{H}_{4}, 4-\mathrm{Cl}_{-} \mathrm{C}_{6} \mathrm{H}_{4}, 4-\mathrm{F}-\mathrm{C}_{6} \mathrm{H}_{4}, 4-\mathrm{HO}-\mathrm{C}_{6} \mathrm{H}_{4}, 4-\mathrm{O}_{2} \mathrm{~N}^{-} \mathrm{C}_{6} \mathrm{H}_{4}, \mathrm{CH}_{3}, 2-$ Thienyl; $\mathrm{R}^{3}=\mathrm{H}, \mathrm{C}_{6} \mathrm{H}_{5} ; \boldsymbol{i}=\mathrm{PEG}-400 /$ Stirr $40{ }^{\circ} \mathrm{C} 2 \mathrm{hrs}$, EtOH/ Pip. / reflux

Scheme 102. Synthesis of pyrano[2,3-c]pyrazole derivatives 225.

Reaction of pyrazolone derivatives 111 with 2-((5-chloro-1,3-diphenyl-1H-pyrazol-4-yl)methylene)malononitrile 54 afforded the corresponding 6-amino-1-1,4-dihydropyrano[2,3-c]pyrazole-5-carbonitriles 226 ${ }^{161,162}$. On the other hand, Abderazek et al. ${ }^{161}$ reported that heating of pyrazolone derivatives 111 with 54 in ethanol containing piperidine afforded a mixture of 6-amino-1-phenyl-1,4-dihydropyrano[2,3-c]pyrazole-5-carbonitriles 227 and 1,7-diphenyl-4,7-dihydro-1H-pyrano[2,3-c;6,5-c9]dipyrazoles 228 (Scheme 103). 


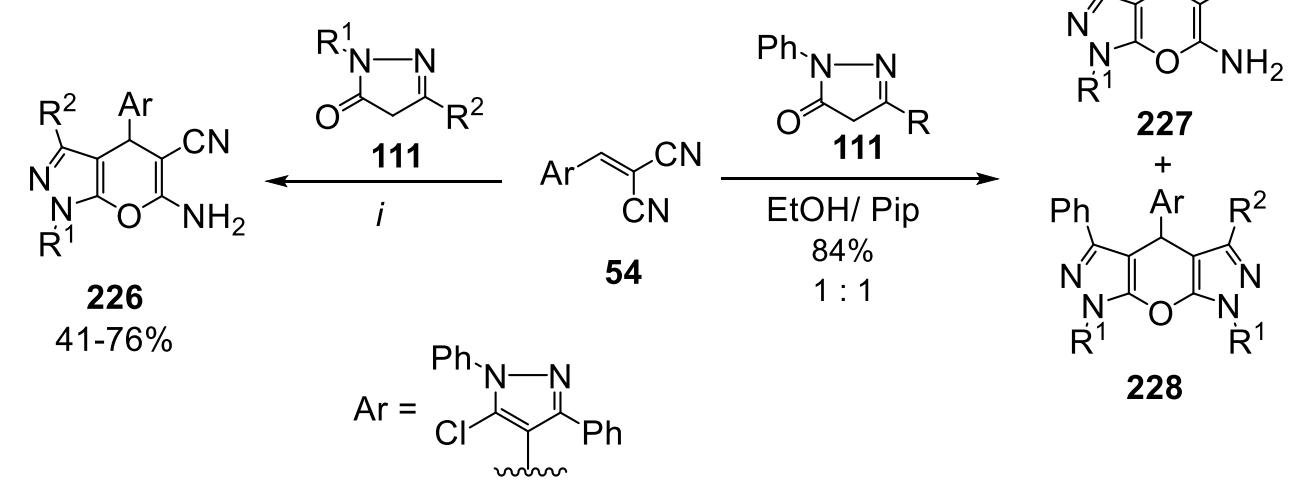

$\mathrm{R}^{1}=\mathrm{H}, \mathrm{C}_{6} \mathrm{H}_{5} ; \mathrm{R}^{2}=\mathrm{CH}_{3}, \mathrm{C}_{6} \mathrm{H}_{5} ; \boldsymbol{i}=\mathrm{EtOH} /$ Pip. /Reflux 3hr, ${ }^{161,162} \mathrm{EtOH} / \mathrm{NaOEt} .{ }^{161}$

Scheme 103. Synthesis of a mixture of pyrano[2,3-c]pyrazoles $\mathbf{2 2 7}$ and pyrano[2,3-c;6,5-c9]dipyrazoles 228.

3.2.1.1.6.2. Pyrano[2,3- $d]$ thiazole derivatives. Abdelrazek et al. ${ }^{161}$ reported that the reaction of 2-((5-chloro1,3-diphenyl-1H-pyrazol-4-yl)methylene)malononitrile 54 with substituted thiazolin-4-ones 199 afforded pyrano[2,3-d] thiazole derivatives 229 but not thiazolo[3,2-a]pyridine derivatives 203 (Scheme 104).

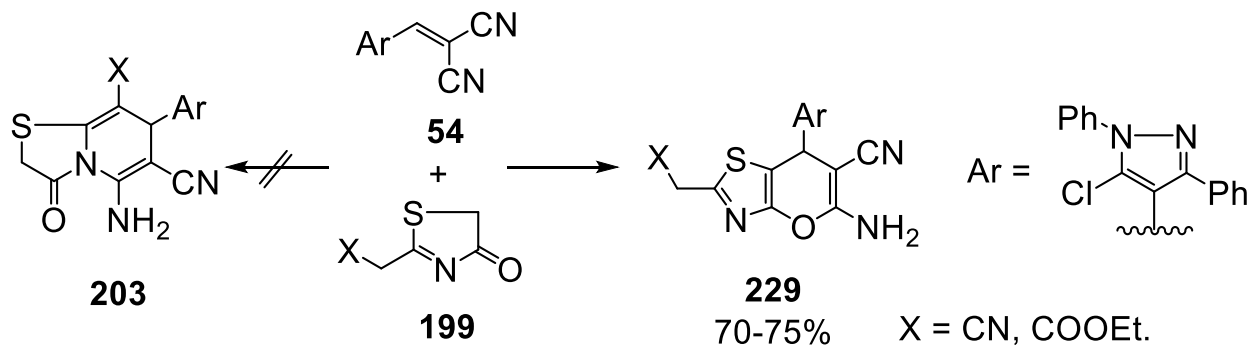

Scheme 104. Synthesis of pyrano[2,3-d]thiazole derivatives 229.

3.2.1.1.6.3. Thiopyrano[2,3-d]thiazole derivatives. Metwally et al. ${ }^{209}$ reported that the knöevenagel condensation of 3-phenyl-4-thioxo-2-thiazolidinone $\mathbf{2 3 0}$ with 1-phenyl-3-aryl-1H-pyrazole-4-carboxaldehydes 2 in glacial acetic acid at reflux or in PEG-400 at room temperature without a catalyst afforded the corresponding 5-pyrazolylmethylene derivatives 231. [4+2] Cycloaddition reaction of compounds 231 with acrylonitrile and ethyl acrylate $\mathbf{2 3 2}$ afforded the corresponding thiopyrano[2,3-d] thiazole derivatives 233 (Scheme 105).

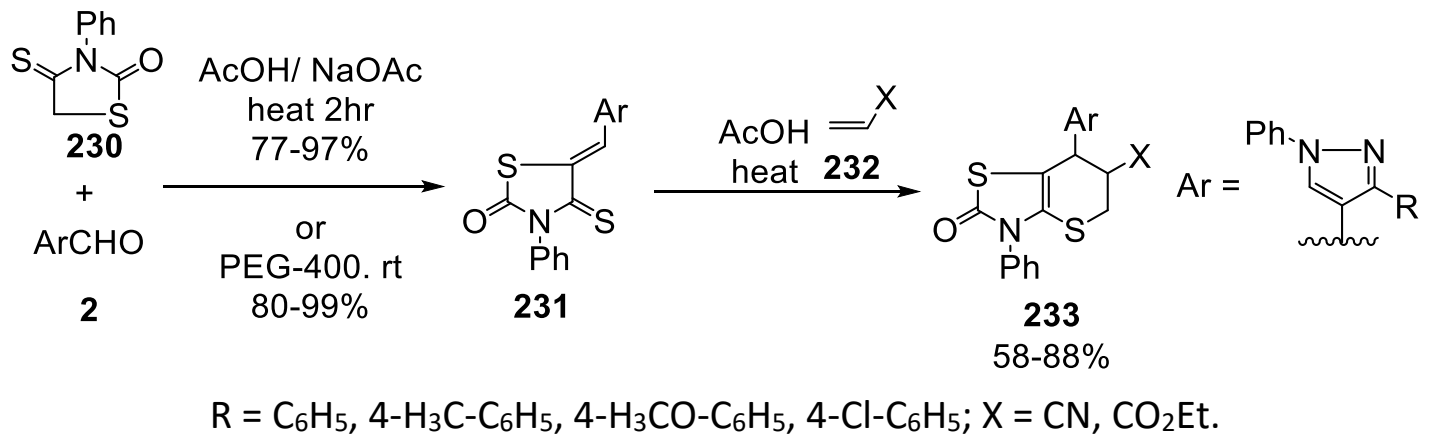

Scheme 105. Synthesis of thiopyrano[2,3-d]thiazole derivatives 233. 
3.2.1.1.7. Fused [6-6] system with one heteroatom. 3.2.1.1.7.1. Chromene derivatives. Oxidative cyclization of pyrazolylpropenones 42 using copper chloride in DMSO ${ }^{105,210}$ or hydrogen peroxide $\left(\mathrm{H}_{2} \mathrm{O}_{2}\right)$ in potassium hydroxide solution in methanol by Algar Flynn Oymanda (AFO) reaction ${ }^{211}$ gave chlorochromones 234 (Scheme 106).

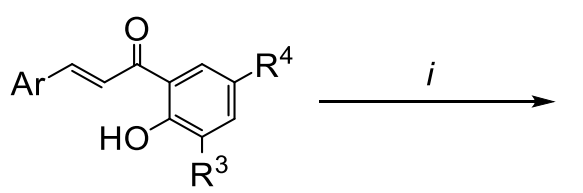

42

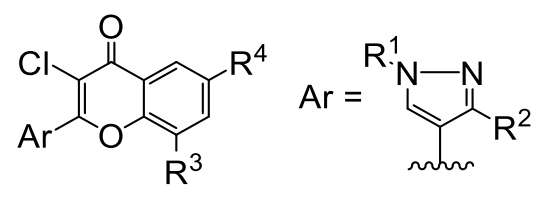

234

$i=\mathrm{DMSO} /$ excess $\mathrm{CuCl}_{2} /$ Reflux, $\mathrm{KOH} / \mathrm{CH}_{3} \mathrm{OH} / \mathrm{H}_{2} \mathrm{O}_{2}$

$\mathrm{R}^{1}=\mathrm{C}_{6} \mathrm{H}_{5}, \mathrm{CH}_{3} ; \mathrm{R}^{2}=\mathrm{C}_{6} \mathrm{H}_{5}, 4-\mathrm{H}_{3} \mathrm{C}-\mathrm{C}_{6} \mathrm{H}_{4}, 4-\mathrm{Cl}-\mathrm{C}_{6} \mathrm{H}_{4}, 4-\mathrm{Br}-\mathrm{C}_{6} \mathrm{H}_{4}, 4-\mathrm{O}_{2} \mathrm{~N}-\mathrm{C}_{6} \mathrm{H}_{4}, 4-\mathrm{H}_{3} \mathrm{CO}-\mathrm{C}_{6} \mathrm{H}_{5}, 4-\mathrm{F}-\mathrm{C}_{6} \mathrm{H}_{4} ; \mathrm{R}^{3}=\mathrm{H}, \mathrm{CH}_{3} ; \mathrm{R}^{4}=$ $\mathrm{H}, \mathrm{Cl}, \mathrm{CH}_{3}, \mathrm{Br}, \mathrm{F}$.

Scheme 106. Synthesis of chlorochromones 234.

El-Emary et al. ${ }^{166}$ reported that the treatment of hydrazone derivative $\mathbf{5 8}$ with salicylaldehyde $\mathbf{4 3}$ yielded chromene 235 (Scheme 107).<smiles>N#CCC(=O)NN=CBr</smiles>

58<smiles>O=Cc1ccccc1O</smiles>

43

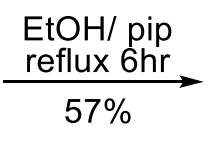

$57 \%$<smiles>N=c1oc2ccccc2cc1C(=O)NN=CI</smiles>

235

Scheme 107. Synthesis of chromene 235.

A series of 4-pyrazolyl-4H-benzopyranes 237 has been synthesized via a one-pot three-component cyclocondensation reaction of 1-phenyl-3-(het)aryl-pyrazole-4-carboxaldehyde $\mathbf{2}$, malononitrile 53, and dimedone $\mathbf{2 3 6}$ in the presence of (diacetoxyiodo)benzene ${ }^{212}$ or piperidine as catalysts ${ }^{208}$ (Scheme 108).

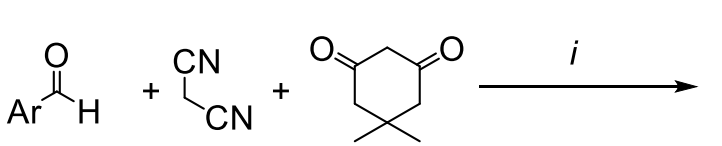

2
53
236<smiles>CC1(C)CC(=O)C2=C(C1)OC(N)=C(C#N)C2Br</smiles>

237 $58-90 \%$<smiles>[R]c1nn([R1])cc1C</smiles><smiles></smiles>

$\mathrm{R}^{1}=\mathrm{C}_{6} \mathrm{H}_{5} ; \mathrm{R}^{2}=\mathrm{C}_{6} \mathrm{H}_{5}, 4-\mathrm{Br}-\mathrm{C}_{6} \mathrm{H}_{4}, 4-\mathrm{Cl}-\mathrm{C}_{6} \mathrm{H}_{4}, 4-\mathrm{F}-\mathrm{C}_{6} \mathrm{H}_{4}, 4-\mathrm{H}_{3} \mathrm{CO}-\mathrm{C}_{6} \mathrm{H}_{4}, 4-\mathrm{H}_{3} \mathrm{C}-\mathrm{C}_{6} \mathrm{H}_{4}, 4-\mathrm{O}_{2} \mathrm{~N}-\mathrm{C}_{6} \mathrm{H}_{4}, 2-\mathrm{Thienyl} ; \boldsymbol{i}=$ (diacetoxyiodo)benzene $(5 \mathrm{~mol} \%) / \mathrm{EtOH} /$ reflux, $^{212} \mathrm{EtOH} / \mathrm{Pip} . / \mathrm{reflux}^{208}$

Scheme 108. Synthesis of 4-pyrazolyl-4H-benzopyranes 237.

3.2.1.1.7. 2. Quinoline derivatives. Multi-component reaction of pyrazole-4-carboxaldehydes 2 , dimedone ${ }^{213-}$ 215 or 1,3-cyclohexanedione 215236 and methyl-3-aminobut-2-enoate ${ }^{213} \mathbf{2 3 8}$ or ethylacetoacetate 214,215 64 under various conditions afforded hexahydroquinoline derivatives 239 (Scheme 109). 


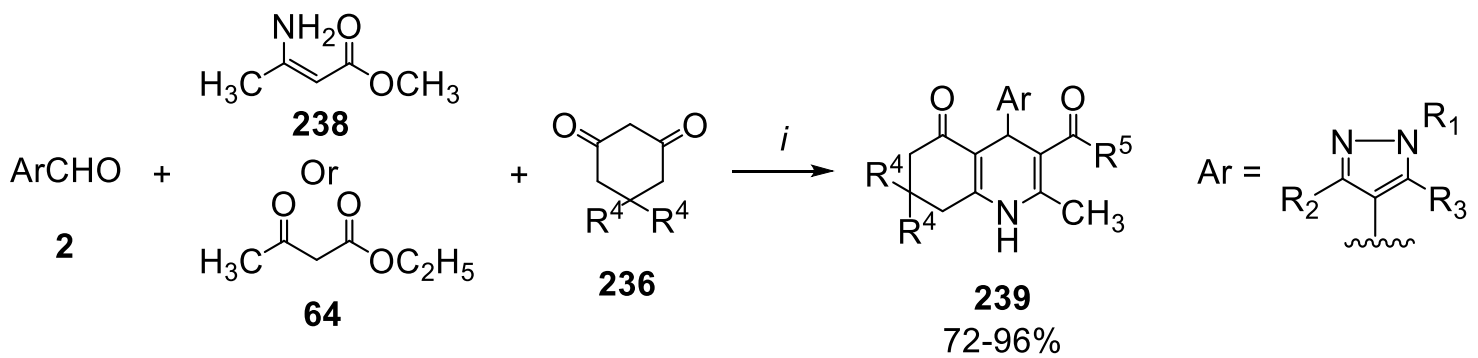

$\mathrm{R}^{1}=\mathrm{C}_{6} \mathrm{H}_{5}, \mathrm{H}, 2,4-\mathrm{di}-\mathrm{O}_{2} \mathrm{~N}-\mathrm{C}_{6} \mathrm{H}_{3} ; \mathrm{R}^{2}=\mathrm{CH}_{3}, 4-\mathrm{Cl}-\mathrm{C}_{6} \mathrm{H}_{4}, 4-\mathrm{H}_{3} \mathrm{C}-\mathrm{C}_{6} \mathrm{H}_{4}, 4-\mathrm{O}_{2} \mathrm{~N}-\mathrm{C}_{6} \mathrm{H}_{4} ; \mathrm{R}^{3}=\mathrm{Cl}, \mathrm{H} ; \mathrm{R}^{4}=\mathrm{H}, \mathrm{CH}_{3} ; \mathrm{R}^{5}=\mathrm{OCH}_{3},{ }^{213}$ $\mathrm{OC}_{2} \mathrm{H}_{5},{ }^{214}, 215 \boldsymbol{i}=\mathrm{Pip}$. / EtOH, ${ }^{213} \mathrm{NH}_{4} \mathrm{OAc} /$ Poly(4-vinylpyridinium)hydrogen sulfate $\left[\mathrm{P}(4-\mathrm{VPH}) \mathrm{HSO}_{4}\right] / \mathrm{H}_{2} \mathrm{O} / \mathrm{reflux}$ 5-15 min, ${ }^{214} \mathrm{NH}_{4} \mathrm{OAc} /$ Cellulose-sulforic acid/ EtOH/ reflux. ${ }^{215}$

Scheme 109. Synthesis of hexahydroquinoline derivatives 239.

Heating a mixture of pyrazole-4-carboxaldehyde $\mathbf{2}$, malononitrile $\mathbf{5 3}$, and the appropriate $\mathbf{6}$-enaminones 240 in acetonitrile containing piperidine at reflux led to the formation of the corresponding 2-amino-5-oxo1,4,5,6,7,8-hexahydroquinoline-3-carbonitrile $\mathbf{2 4 1 ^ { 2 1 6 }}$. Moreover, the reaction of pyrazole-4-carboxaldehyde $\mathbf{2}$, Meldrum's acid 242, and the appropriate 6 -enaminones $\mathbf{2 4 0}$ in acetonitrile containing few drops of piperidine at reflux afforded the corresponding 7,7-disubstituted-3,4,7,8-tetrahydroquinoline-2,5(1H,6H)-dione $243^{217}$ (Scheme 110).

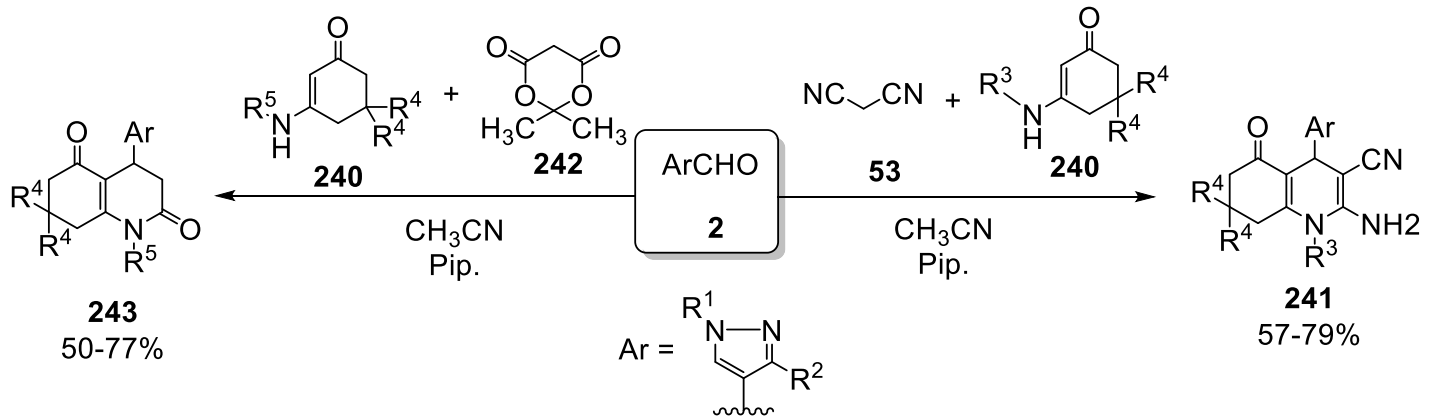

$\mathrm{R}^{1}=\mathrm{C}_{6} \mathrm{H}_{5} ; \mathrm{R}^{2}=\mathrm{C}_{6} \mathrm{H}_{5}, 4-\mathrm{Br}-\mathrm{C}_{6} \mathrm{H}_{4}, 4-\mathrm{Cl}-\mathrm{C}_{6} \mathrm{H}_{4}, 4-\mathrm{F}-\mathrm{C}_{6} \mathrm{H}_{4}, 4-\mathrm{H}_{3} \mathrm{CO}-\mathrm{C}_{6} \mathrm{H}_{4}, 4-\mathrm{H}_{3} \mathrm{C}-\mathrm{C}_{6} \mathrm{H}_{4}, 4-\mathrm{O}_{2} \mathrm{~N}-\mathrm{C}_{6} \mathrm{H}_{4}$, 2-Thienyl; $\mathrm{R}^{3}=4-(4-$ $\mathrm{F}-\mathrm{C}_{6} \mathrm{H}_{4}$ ) thiazol-2-yl, 4-F- $\mathrm{C}_{6} \mathrm{H}_{4} ; \mathrm{R}^{4}=\mathrm{H}, \mathrm{CH}_{3} ; \mathrm{R}^{5}=4-\left(4-\mathrm{F}-\mathrm{C}_{6} \mathrm{H}_{4}\right)$ thiazol-2-yl.

Scheme 110. Synthesis of hexahydroquinoline-3-carbonitriles $\mathbf{2 4 1}$ and tetrahydroquinoline-2,5-diones 243.

3.2.1.7.1.3. Isoquinoline derivatives. Nandakumar and Perumal ${ }^{218}$ reported that the coupling reactions of $N$ benzyl-1-(2-bromo-4,5-dimethoxyphenyl)methanamine 244, 1,3-diphenyl-1H-pyrazole-4-carboxaldehyde 2 and 1-ethynyl-2-phenoxybenzene $\mathbf{2 4 5}$ using copper(I) iodide (Cul) as a catalyst afforded propargyl amine derivative 246 which underwent intramolecular carbocyclization to give tetrahydroisoquinoline derivative 247 (Scheme 111). 


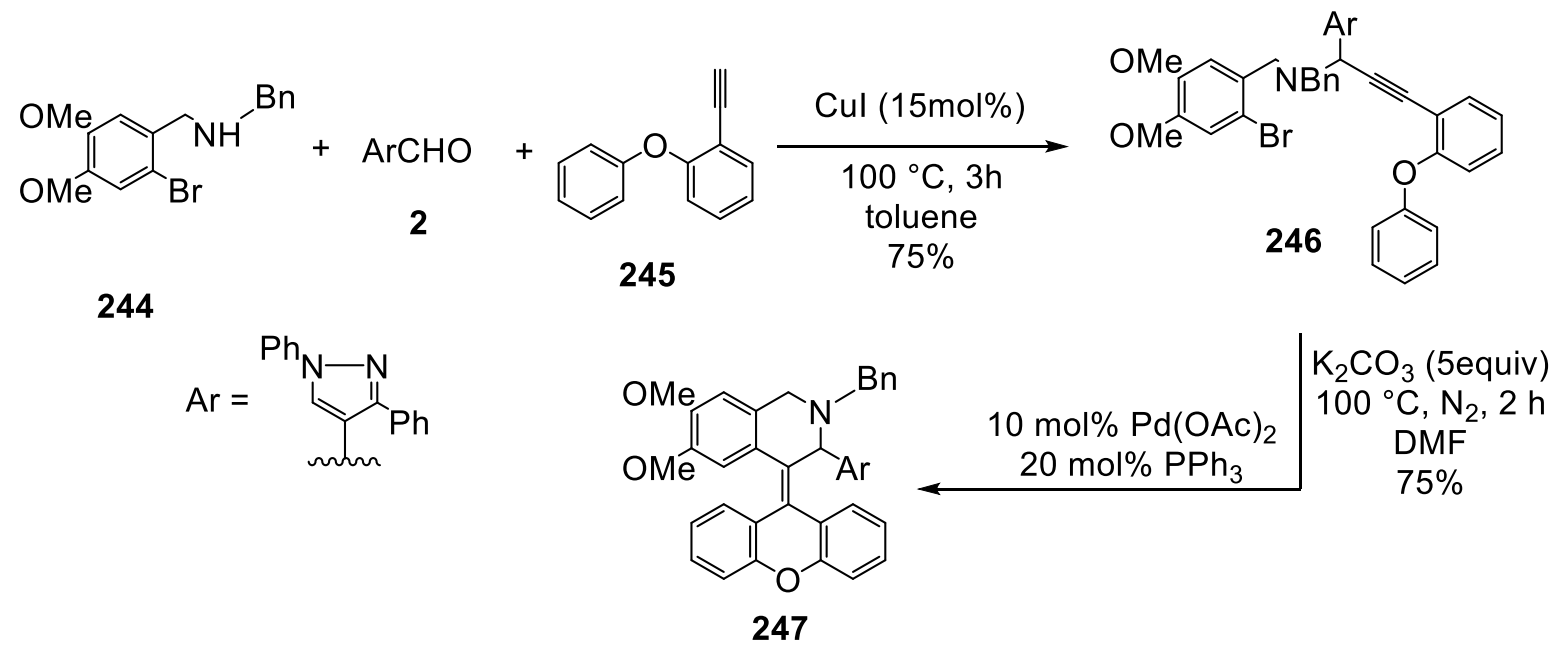

Scheme 111. Synthesis of tetrahydroisoquinoline derivative 247.

3.2.1.1.8. Fused [6-6] system with two heteroatoms. 3.2.1.1.8.1. Quinazoline derivatives. Biginelli condensation of cyclohexane-1,3-dione ${ }^{120,219}$ or 5,5-dimethyl-1,3-cyclohexanedione 220236 , (thiourea or urea 184) and pyrazole-4-carboxaldehyde 2 in methanol at reflux afforded 2,3,4,6,7,8-hexahydroquinazolin-5(1H)one or 4,6,7,8-tetrahydroquinazoline-2,5(1H,3H)-dione derivatives $\mathbf{2 4 8 .}$. $^{120,219,220}$ Similarly, condensation of cyclohexanone 249, (thiourea or urea 184) and 1,3-diphenyl-1H-pyrazole-4-carboxaldehyde 2 in methanol at reflux afforded 3,4,5,6,7,8-hexahydroquinazoline-2(1H)-thione or 3,4,5,6,7,8-hexahydroquinazolin-2(1H)-one analogues $\mathbf{2 5 0 ^ { 2 1 9 }}$ (Scheme 112).

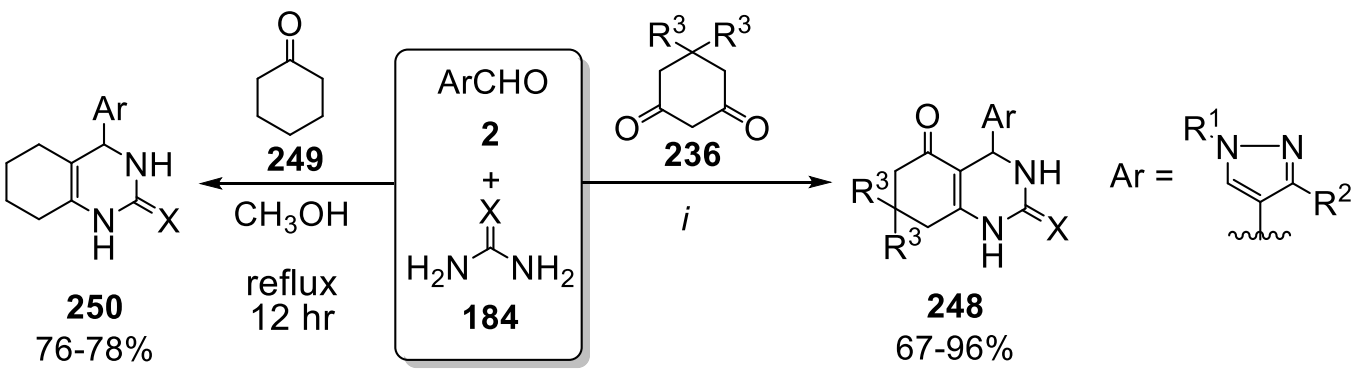

$\mathrm{R}^{1}=\mathrm{C}_{6} \mathrm{H}_{5}, 3-\mathrm{Cl}-\mathrm{C}_{6} \mathrm{H}_{4}, \mathrm{H} ; \mathrm{R}^{2}=\mathrm{C}_{6} \mathrm{H}_{5}, 4-\mathrm{H}_{3} \mathrm{CO}-\mathrm{C}_{6} \mathrm{H}_{4}, 4-\mathrm{F}-\mathrm{C}_{6} \mathrm{H}_{4}, 4-\mathrm{Cl}_{-}-\mathrm{C}_{6} \mathrm{H}_{4} ; \mathrm{R}^{3}=\mathrm{H}, \mathrm{CH}_{3} ; \mathrm{X}=\mathrm{O}, \mathrm{S} ; \boldsymbol{i}=$ reflux/ methanol, ${ }^{219}$ drops of $\mathrm{HCl} / \mathrm{EtOH}$, refux, ${ }^{120} p-\mathrm{TsOH} / \mathrm{H}_{2} \mathrm{O} /$ reflux. $^{220}$

Scheme 112. Synthesis of hexahydroquinazolin-5(1H)-ones and hexahydroquinazoline-2(1H)-thiones 248 and 250.

Kamble et al. ${ }^{68}$ reported the synthesis of 3-benzyl-2,3-dihydro-2-(1-phenyl-3-substituted-1H-pyrazol-4yl)quinazolin-4(1H)-ones 252 via the reaction of 3-(substituted)-1-phenyl-1H-pyrazole-4-carboxaldehydes 2 with aminobenzamide $\mathbf{2 5 1}$ upon heating in methanol at reflux in the presence of potassium carbonate (Scheme 113).<smiles>Nc1ccccc1C(=O)NCc1ccccc1</smiles>

251

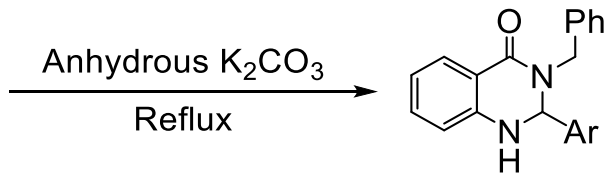

252<smiles>[R]c1nn([R1])cc1C</smiles> 


$$
\mathrm{R}^{1}=\mathrm{C}_{6} \mathrm{H}_{5} ; \mathrm{R}^{2}=\mathrm{C}_{6} \mathrm{H}_{5}, 3-\mathrm{Br}-\mathrm{C}_{6} \mathrm{H}_{4}, 4-\mathrm{Cl}-\mathrm{C}_{6} \mathrm{H}_{4}, 4-\mathrm{F}-\mathrm{C}_{6} \mathrm{H}_{4}, 4-\mathrm{O}_{2} \mathrm{~N}-\mathrm{C}_{6} \mathrm{H}_{4} \text {, Pyridine-3-yl, Thiophen-2-yl. }
$$

Scheme 113. Synthesis of 3-benzyl-2-(1-phenyl-3-substituted-1H-pyrazol-4-yl)quinazolin-4(1H)-ones 252.

Nikpassand et al. ${ }^{221}$ reported that pyrazolyl-substituted quinazolinones 252 were obtained via a threecomponent condensation of pyrazole-4-carboxaldehyde $\mathbf{2}$ with isatoic anhydride $\mathbf{2 5 3}$ and ammonium acetate under microwave irradiation in the presence of [BDBIm] $\mathrm{Br}$ as a catalyst which could be recovered easily and reused without appreciable loss of reactivity (Scheme 114).

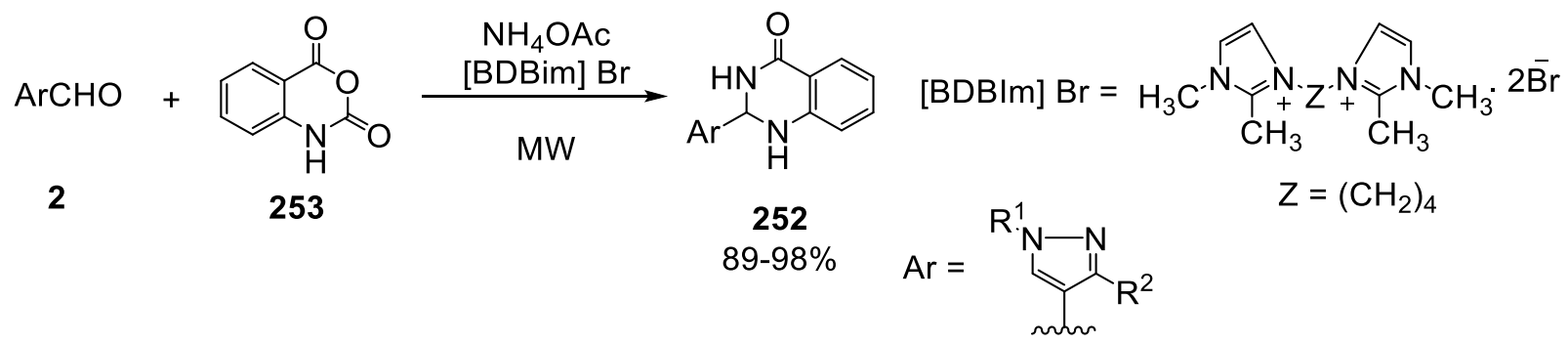

[BDBIm] Br: 3,3'-(butane-1,4-diyl)bis(1,2-dimethyl-1H-imidazol-3-ium) dibromide.

$$
\mathrm{R}^{1}=\mathrm{C}_{6} \mathrm{H}_{5} ; \mathrm{R}^{2}=4-\mathrm{H}_{3} \mathrm{CO}-\mathrm{C}_{6} \mathrm{H}_{4}, 4-\mathrm{HO}-\mathrm{C}_{6} \mathrm{H}_{4}, 4-\mathrm{Cl}-\mathrm{C}_{6} \mathrm{H}_{4}, 2-\mathrm{HO}-\mathrm{C}_{6} \mathrm{H}_{4}, \mathrm{C}_{6} \mathrm{H}_{5} \text {. }
$$

Scheme 114. Synthesis of pyrazolyl-substituted quinazolinones 252.

Mehta et al. $^{222}$ reported the synthesis of a series of quinazolin-4(3H)-one derivatives 252 containing a (1,3-diph0enyl-1H-pyrazol-4-yl) substituent at the position-2 and aromatic or heteroaromatic substituents at the position-3 by using /-proline 254 to catalyze the one-pot multi-component reaction of 1-phenyl-3-aryl- $1 \mathrm{H}$ pyrazole-4-carboxaldehyde 2, isatioc anhydride 253, aromatic amines $\mathbf{7 7}$ in methanol at reflux (Scheme 115).

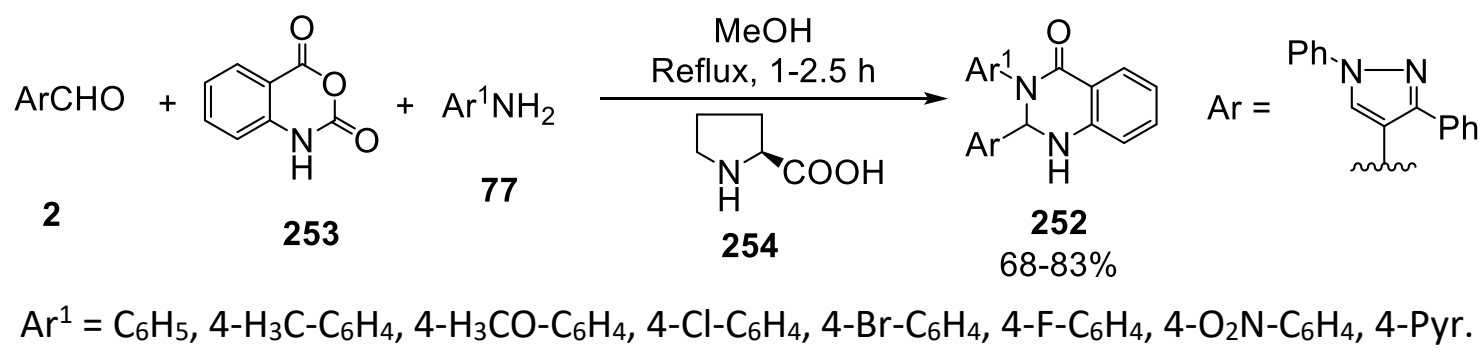

Scheme 115. Synthesis of pyrazolyl-substituted quinazolinones 252.

3.2.1.1.8.2. Phthalazine derivatives. The reaction of ethyl-5-cyano-4-methyl-6-oxo-1-phenyl-1,6dihydropyridazine-3-carboxylate $\mathbf{2 5 5}$ with pyrazolylmethylene malononitrile $\mathbf{5 4}$ gave the phthalazinone derivative $\mathbf{2 5 6}^{161,162}$ (Scheme 116).

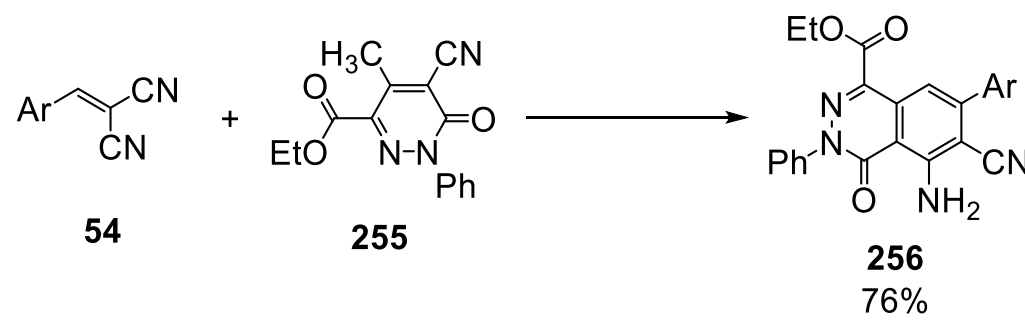<smiles>Clc1c(-c2ccccc2)nn(-c2ccccc2)c1Cl</smiles> 
Scheme 116. Synthesis of phthalazinone derivative 256.

3.2.1.1.9. Fused [6-6] system with three heteroatoms. 3.2.1.1.9.1. Pyridopyrimidine derivatives. Nia et al..$^{223}$ reported that stirring equimolar amounts of 6-amino-2-(methylthio)pyrimidin-4(3H)-one 257, Meldrum's acid 242, and pyrazole-4-carboxaldehydes 2 with a catalytic amount of 1,2-dimethyl- $N$-butanesulfonic acid imidazolium hydrogen sulfate $[\mathrm{DMBSI}] \mathrm{HSO}_{4}$ in an oil bath led to the formation of the corresponding pyridopyrimidine derivatives 258 (Scheme 117).

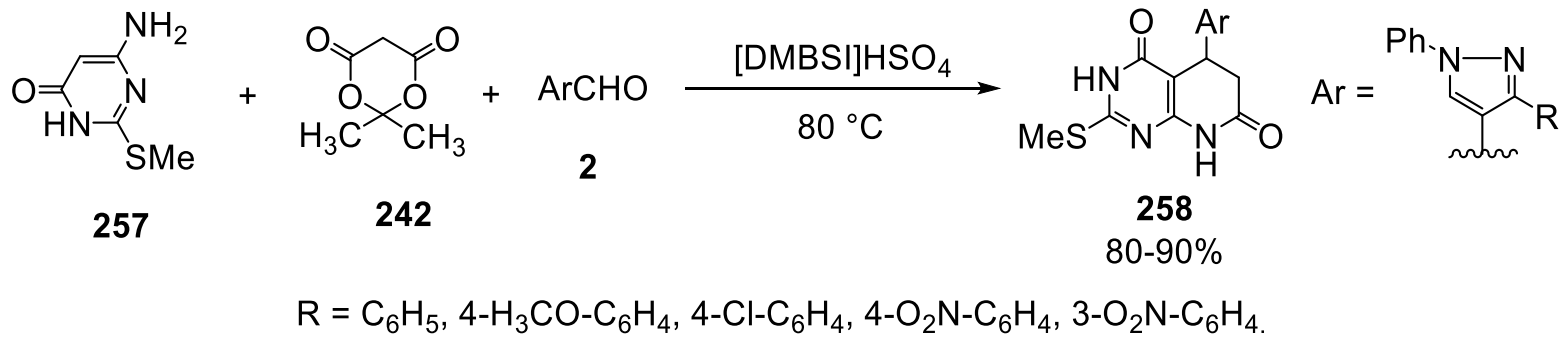

Scheme 117. Synthesis of pyridopyrimidine derivatives 258.

3.2.1.9.2. Benzo[e][1,4,2](ox/ di/ thi)azaphosphinine derivatives. Reaction of 1,3-diphenyl-1H-pyrazole-4carboxaldehyde 2 with 2-aminophenol 221, 1,2-phenylenediamine 68 and 2-aminothiophenol 223 in the presence of diethyl phosphite $\mathrm{H}-\mathrm{P}(\mathrm{O})(\mathrm{OEt})_{2} 125$ and sodium hydride gave 3-(1,3-diphenyl-1H-pyrazol-4-yl)-2ethoxy-3,4-dihydrobenzo[e][1,4,2](ox/ di/ thi)azaphosphinine-2-oxide derivatives 259 (Scheme 118) .117

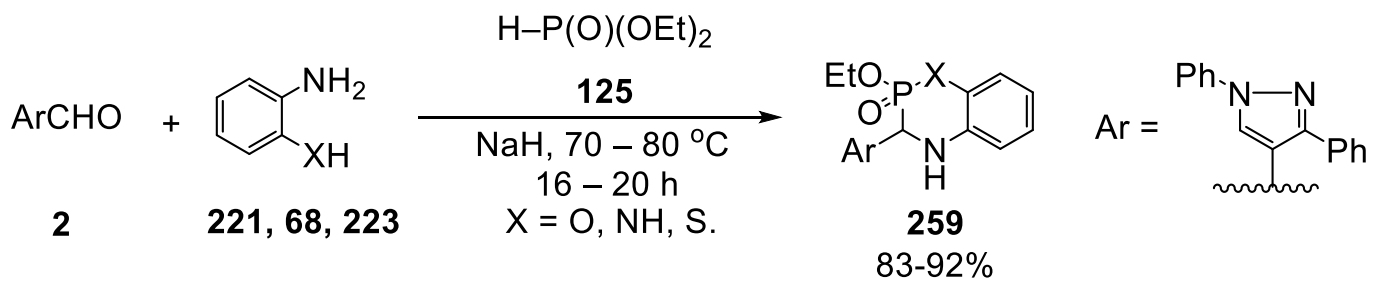

Scheme 118. Synthesis of 3-(1H-pyrazol-4-yl)-3,4-dihydrobenzo[e][1,4,2](ox/di/thi)azaphosphinine-2-oxide derivatives 259.

3.2.1.1.10. Fused [6-6] system with four heteroatoms. 3.2.1.1.10.1. Pyrimido[4,5- $d$ ] pyrimidine derivatives. Suresh et al. ${ }^{224}$ reported that a four-component reaction of 6-amino-1,3-dimethyluracil 260, $N, N$ dimethylformamide dimethyl acetal 261, 1-phenyl-3-(4-substituted-phenyl)-4-formyl-1Hpyrazoles 2 and aromatic amines $\mathbf{7 7}$ in the presence of 1-butyl-3-methylimidazolium tetrachloroferrate $\left[\mathrm{Bmim}^{\mathrm{FeCl}} \mathrm{F}_{4}\right.$ ionic liquid as a promoting medium gave pyrazolopyrimido[4,5-d]pyrimidines derivatives 262 (Scheme 119). 


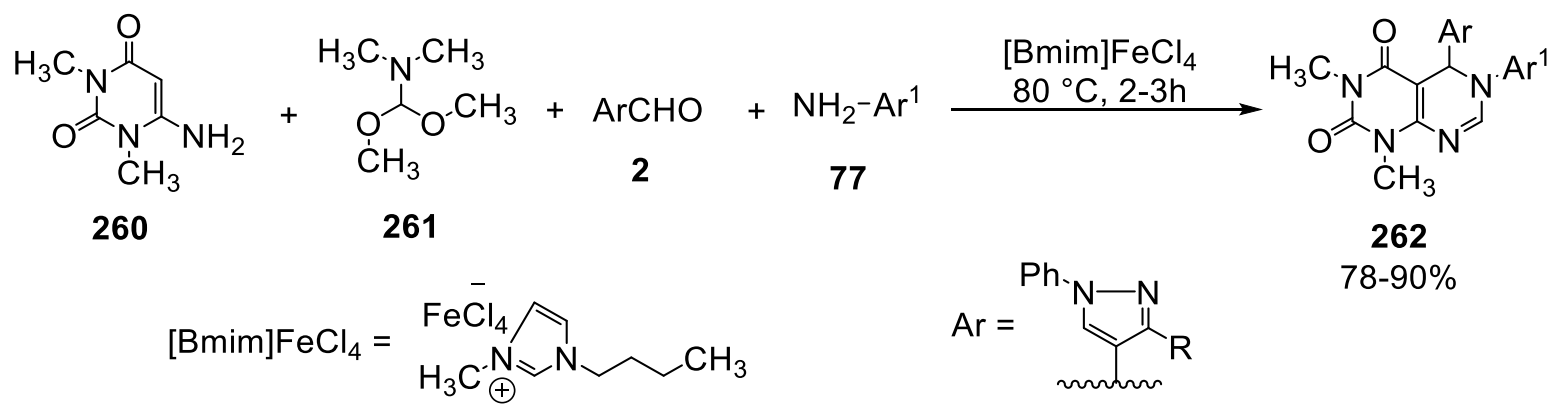

$\mathrm{R}=4-\mathrm{O}_{2} \mathrm{~N}-\mathrm{C}_{6} \mathrm{H}_{4}, 4-\mathrm{Cl}-\mathrm{C}_{6} \mathrm{H}_{4}, 4-\mathrm{H}_{3} \mathrm{C}-\mathrm{C}_{6} \mathrm{H}_{4}, \mathrm{C}_{6} \mathrm{H}_{5}$, Thiophene; $\mathrm{Ar}^{1}=\mathrm{C}_{6} \mathrm{H}_{5}, 4-\mathrm{Cl}_{-}-\mathrm{C}_{6} \mathrm{H}_{4}, 4-\mathrm{H}_{3} \mathrm{C}-\mathrm{C}_{6} \mathrm{H}_{4}, 4-\mathrm{H}_{3} \mathrm{CO}-\mathrm{C}_{6} \mathrm{H}_{4}, 3-\mathrm{O}_{2} \mathrm{~N}-$ $\mathrm{C}_{6} \mathrm{H}_{4}$.

Scheme 119. Synthesis of pyrazolopyrimido[4,5-d]pyrimidines derivatives 262.

3.2.1.1.11. Fused [6-7] system with two heteroatoms. 3.2.1.1.11.1. Benzo[b][1,4]thiazepine derivatives. Karale et al. ${ }^{210}$ reported that the condensation of pyrazolylpropenones $\mathbf{4 2}$ with 2-aminothiophenol 223 in ethanol containing acetic acid at reflux gave benzothiazepines 263 (Scheme 120).

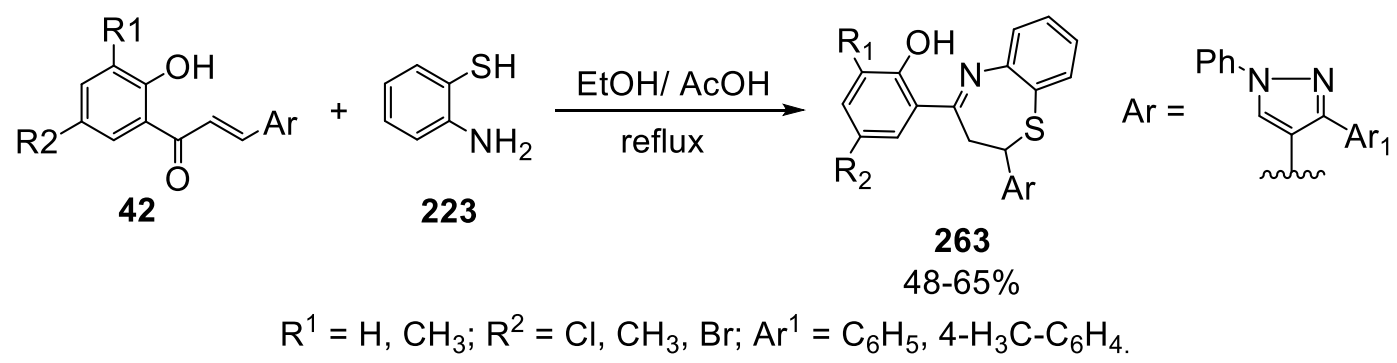

Scheme 120. Synthesis of benzothiazepines 263.

3.2.2. Pyrazole-substituted tricyclic system. 3.2.2.1. Fused [5-5-6] system with three heteroatoms. 3.2.2.1.1. Cyclopenta[b]pyrazolo[4,3-e]pyridine derivatives. Lipson et al. ${ }^{225}$ reported that heating of equimolar quantities of 3-methyl-1H-pyrazol-5-amine 204, pyrazole-4-carboxaldehyde 2, and cyclopentane-1,3-dione 264 in 2propanol at reflux led to the formation of the corresponding 3-methyl-4,6,7,8tetrahydrocyclopenta[b]pyrazolo[4,3-e] pyridin-5(2H)-one 265 (Scheme 121).

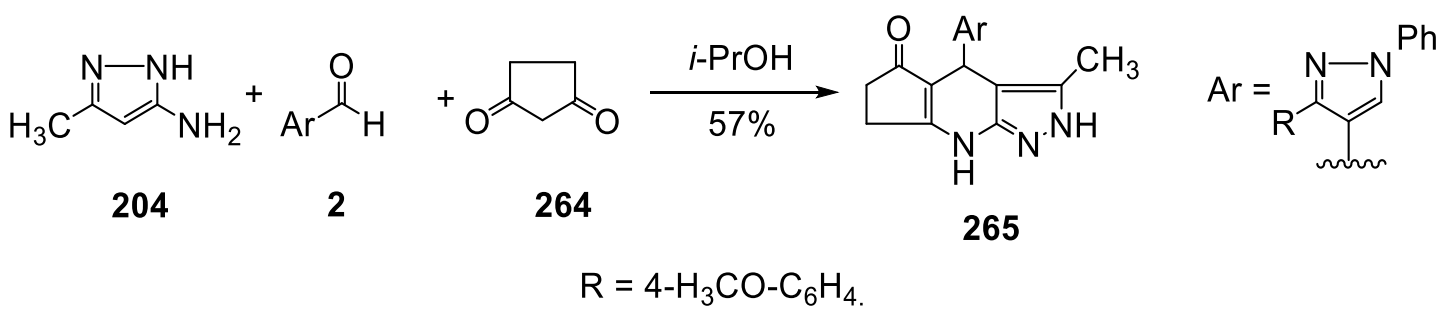

Scheme 121. Synthesis of 3-methyltetrahydrocyclopenta[b]pyrazolo[4,3-e] pyridin-5(2H)-one 265.

3.2.2. Fused [5-5-6] system with five heteroatoms. 3.2.2.2.1. Dipyrazolo[3,4-b:4',3'-e]pyridine derivatives. Dawane et al. ${ }^{226}$ reported that the microwave irradiation of pyrazolone $\mathbf{1 1 1}$, pyrazole-4-carboxaldehydes $\mathbf{2}$, and ammonium acetate 78 dissolved in PEG-400 afforded the corresponding 3,5-dimethyl-1,7-diphenyl-1,4,7,8tetrahydrodipyrazolo[3,4-b:4',3'-e]pyridines 266 (Scheme 122). 


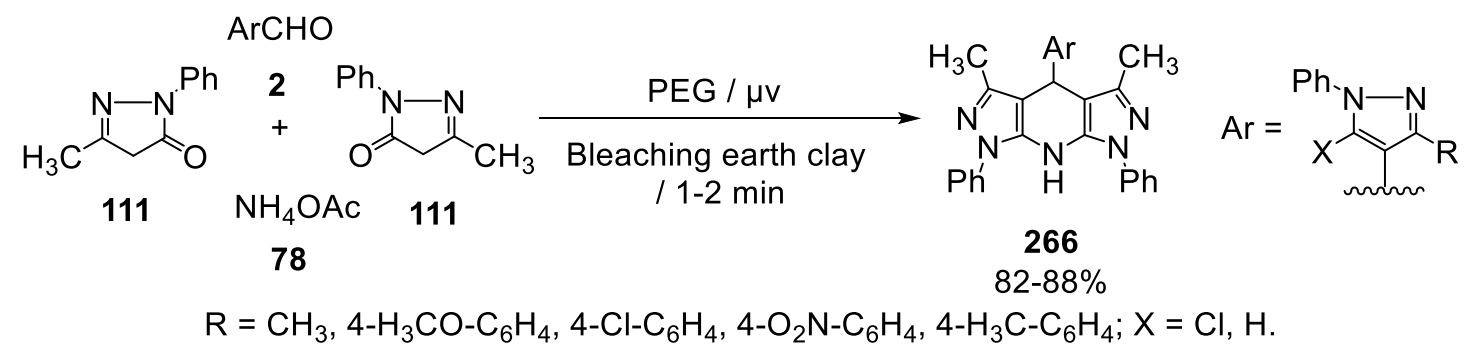

Scheme 122. Synthesis of 1,4,7,8-tetrahydrodipyrazolo[3,4-b:4',3'-e]pyridines 266.

3.2.2.2. Dipyrazolo[1,5-a:3',4'-d]pyrimidine derivatives. Chobe et al. ${ }^{227}$ reported that condensation of 4(arylidene)-3-methyl-1-phenyl-1H-pyrazol-5-ones 112 with 4-((4-chlorophenyl)diazenyl)-3H-pyrazole-3,5diamine 267 in PEG-400 afforded pyrazolo[1,5-a]pyrimidines 268 (Scheme 123).<smiles>CC1=NN(c2ccccc2)C(=O)C1=C[Hg]</smiles>

112

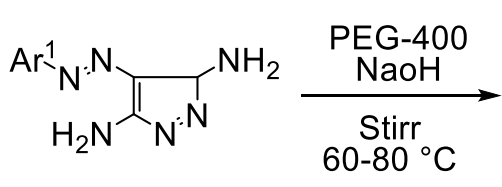

267

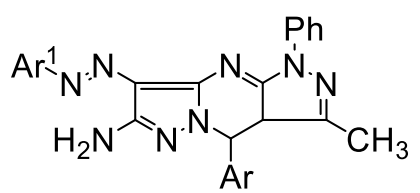

268 $84-86 \%$

$\mathrm{R}=4-\mathrm{H}_{3} \mathrm{CO}-\mathrm{C}_{6} \mathrm{H}_{4}, 4-\mathrm{Cl}-\mathrm{C}_{6} \mathrm{H}_{4}, 4-\mathrm{O}_{2} \mathrm{~N}-\mathrm{C}_{6} \mathrm{H}_{4} ; \mathrm{Ar}{ }^{1}=4-\mathrm{Cl}-\mathrm{C}_{6} \mathrm{H}_{4}$<smiles>[R]c1nn(-c2ccccc2)cc1NC</smiles>

Scheme 123. Synthesis of pyrazolo[1,5-a]pyrimidines 268.

3.2.2.3. Fused [5-6-5] system with three hetero atoms. 3.2.2.3.1. [1,3]Thiazolo[3,2-a]benzimidazole derivatives. One-pot three-component reaction of 1,3-dihydro- $2 \mathrm{H}$-benzimidazole-2-thione 269 with $1,3-$ diphenyl-1H-pyrazole-4-carboxaldehyde 2 and chloroacetic acid 64 in glacial acetic acid and acetic anhydride in the presence of sodium acetate afforded 2-[1H-pyrazol-4-yl-methylene][1,3]thiazolo[3,2-a]benzimidazol-3(2H)one $270^{228}$ (Scheme 124).

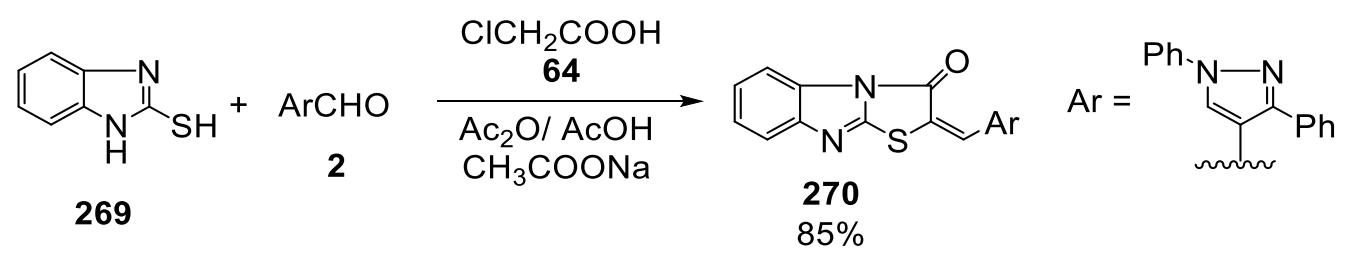

Scheme 124. Synthesis of 2-[1H-pyrazol-4-yl-methylene][1,3]thiazolo[3,2-a]benzimidazolone 270.

3.2.2.3.2. Pyrrolo[3',4':5,6]thiopyrano[2,3-d]thiazole derivatives. [4+2] Cycloaddition reaction of 5pyrazolylmethylene derivatives $\mathbf{2 3 1}$ with $\mathrm{N}$-arylmaleimides $\mathbf{2 7 1}$ in acetic acid at reflux afforded pyrrolo[3', 4':5,6] thiopyrano[2,3-d] thiazole derivatives 272 (Scheme 125). ${ }^{209}$ 

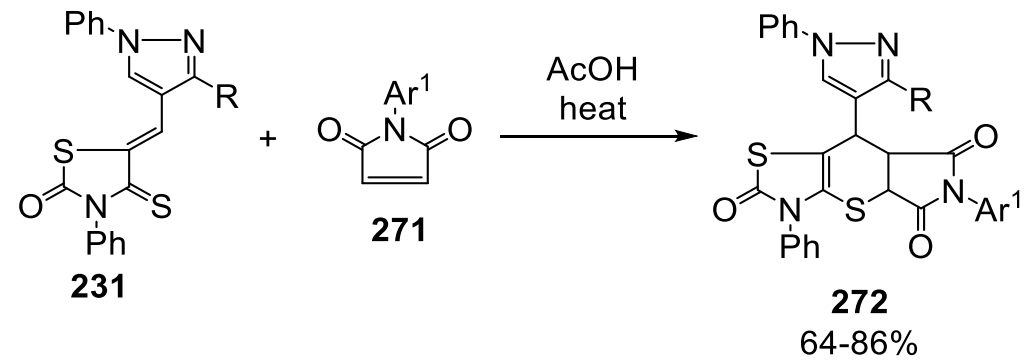

$\mathrm{R}=\mathrm{C}_{6} \mathrm{H}_{5}, 4-\mathrm{H}_{3} \mathrm{C}-\mathrm{C}_{6} \mathrm{H}_{5}, 4-\mathrm{H}_{3} \mathrm{CO}-\mathrm{C}_{6} \mathrm{H}_{5}, 4-\mathrm{Cl}-\mathrm{C}_{6} \mathrm{H}_{5} ; \mathrm{Ar}^{1}=\mathrm{C}_{6} \mathrm{H}_{5}, 4-\mathrm{H}_{3} \mathrm{C}-\mathrm{C}_{6} \mathrm{H}_{5}, 4-\mathrm{H}_{3} \mathrm{CO}-\mathrm{C}_{6} \mathrm{H}_{5}, 4-\mathrm{Cl}-\mathrm{C}_{6} \mathrm{H}_{5}$.

Scheme 125. Synthesis of pyrrolo[3',4':5,6]thiopyrano[2,3-d]thiazole derivatives 272.

3.2.2.4. Fused [5-6-6] system with two heteroatoms. 3.2.2.4.1. Furo[2,3-f]chromene derivatives. Ashok et al. ${ }^{229}$ reported the synthesis of $\{3-[2-(3-a r y l-1-p h e n y l-1 H p y r a z o l-4-y l) v i n y l]-7 H-f u r o[2,3-f] c h r o m e n-2-y l\}-(4-$ bromophenyl) methanones $\mathbf{2 7 3}$ by the reaction of 2-bromo-1-(4-bromophenyl)ethanone 101 with 2hydroxychalcones 42 in acetone containing $\mathrm{K}_{2} \mathrm{CO}_{3}$ under conventional heating, microwave irradiation or ultrasonication (Scheme 126).

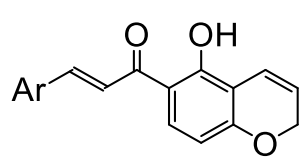

42

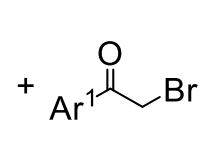

101
Anhydous $\mathrm{K}_{2} \mathrm{CO}_{3}$ dry acetone

$\mathrm{R}^{1}=\mathrm{C}_{6} \mathrm{H}_{5} ; \mathrm{R}^{2}=\mathrm{C}_{6} \mathrm{H}_{5}, 4-\mathrm{Br}_{-} \mathrm{C}_{6} \mathrm{H}_{4}, 4-\mathrm{Cl}-\mathrm{C}_{6} \mathrm{H}_{4}, 4-\mathrm{H}_{3} \mathrm{C}-\mathrm{C}_{6} \mathrm{H}_{4}, 4-\mathrm{H}_{3} \mathrm{CO}-\mathrm{C}_{6} \mathrm{H}_{4}, 4-\mathrm{H}_{5} \mathrm{C}_{2} \mathrm{O}-\mathrm{C}_{6} \mathrm{H}_{4}, 3,4-\mathrm{di}-\left(\mathrm{H}_{3} \mathrm{CO}\right)_{2}-\mathrm{C}_{6} \mathrm{H}_{3}$, Naphth2-yl; $\mathrm{Ar}^{1}=4-\mathrm{Br}_{-} \mathrm{C}_{6} \mathrm{H}_{4} ; \boldsymbol{i}=$ Conventional 54-61\%; Ultrasound 64-74\%; MW 79-85\%

Scheme 126. Synthesis of \{3-[2-(1Hpyrazol-4-yl)vinyl]-7H-furo[2,3-f]chromen-2-yl\}-(4-bromophenyl) methanones 273.

3.2. 2. 2. Pyrazolo[1,2-b]phthalazine derivatives. Shaikh et al. ${ }^{230}$ prepared 3-amino-5,10-dioxo-1-(1-phenyl-3( $p$-tolyl)-1H-pyrazol-4-yl)-5,10-dihydro-1H-pyrazolo[1,2-b]phthalazine-2-carbonitrile 275 by the reaction of 1phenyl-3-(p-tolyl)-1H-pyrazole-4-carboxaldehyde 2, malononitrile 53 and phthalhydrazide 274 catalyzed by 20 mol\% of tributylammonium sulfate $\left[\mathrm{Bu}_{3} \mathrm{NH}\right]\left[\mathrm{HSO}_{4}\right]$ under solvent-free condition (Scheme 127).

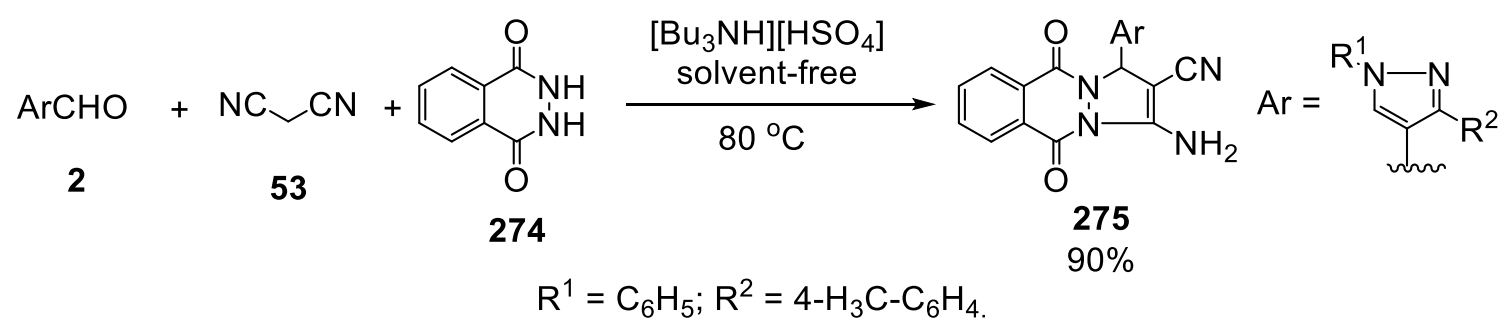

Scheme 127. Synthesis of 1-(1H-pyrazol-4-yl)-5,10-dihydro-1H-pyrazolo[1,2-b]phthalazine-2-carbonitrile 275.

3.2.2.4.3. Pyrrolo[1,2-a]quinoxalines derivatives. Kamal et al. ${ }^{231}$ reported that heating of 1 -(2aminophenyl)pyrrole 276, pyrazole-4-carboxaldehydes 2, and sulfamic acid 277 in $\mathrm{H}_{2} \mathrm{O}$ at reflux afforded dihydropyrrolo[1,2-a]quinoxalines derivatives 278 (Scheme 128). 


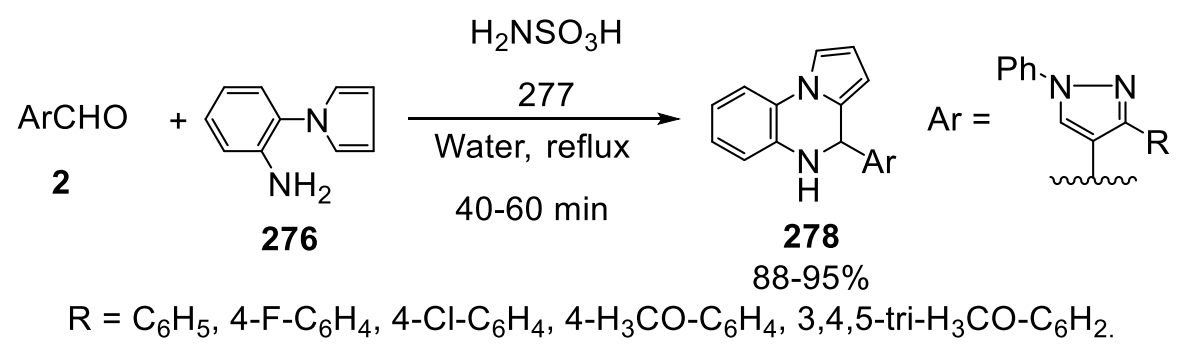

Scheme 128. Synthesis of dihydropyrrolo[1,2-a]quinoxalines derivatives 278.

3.2.2.5. Fused [6-5-5] system with five heteroatoms. 3.2.2.5.1. Pyrano[2,3-c:6,5-c']dipyrazole derivatives. Abdelrazek et al. ${ }^{161}$ reported that the reaction of two equivalents of 2-phenyl-2,4-dihydro-3H-pyrazol-3-ones 111 with 5-chloro-1,3-diphenyl-1H-pyrazole-4-carboxaldehyde 2 afforded the corresponding 1,7-diphenyl-4,7dihydro-1H-pyrano[2,3-c:6,5-c']dipyrazoles 279 (Scheme 129).

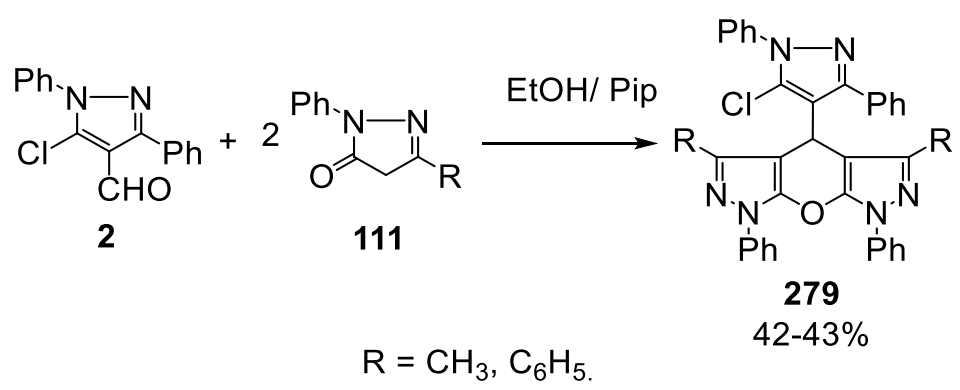

Scheme 129. Synthesis of 1,7-diphenyl-4,7-dihydro-1H-pyrano[2,3-c:6,5-c']dipyrazoles 279.

\subsubsection{Fused [6-5-6] system with one heteroatom. 3.2.2.6.1. Indeno[1,2-b]pyridine derivatives.}

Mamaghani et al. ${ }^{215}$ reported that heating a mixture of 3-aryl-4-formylpyrazole 2, indanedione $\mathbf{2 8 0}$, ethyl acetoacetate $\mathbf{6 4}$, and ammonium acetate $\mathbf{7 8}$ in the presence of cellulose-sulfuric acid in ethanol at reflux furnished 4,5-dihydro- $1 H$-indeno[1,2-b]pyridine-3-carboxylates 281 (Scheme 130).

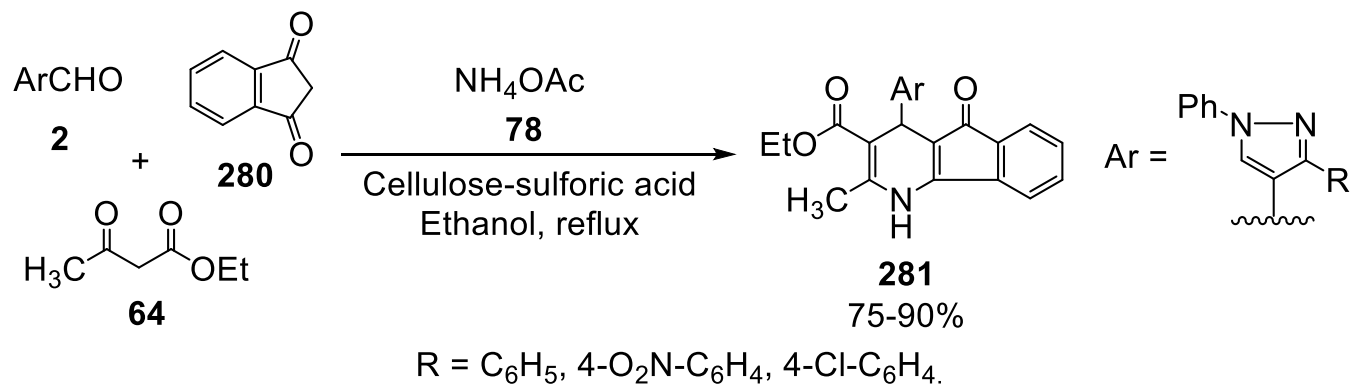

Scheme 130. Synthesis of 4,5-dihydro-1H-indeno[1,2-b]pyridine-3-carboxylates 281.

3.2.7. Fused [6-5-6] system with two heteroatom. 3.2.2.7.1. Indeno[1,2-d]pyrimidine derivatives. Fahmy et al. ${ }^{120}$ reported that the reaction of 1,3-indanedione $\mathbf{2 8 0}$ with urea or thiourea $\mathbf{1 8 4}$ and pyrazole-4carboxaldehydes 2 gave indeno[1,2-d]pyrimidine-2-oxo(thioxo) derivatives 282 (Scheme 131). 


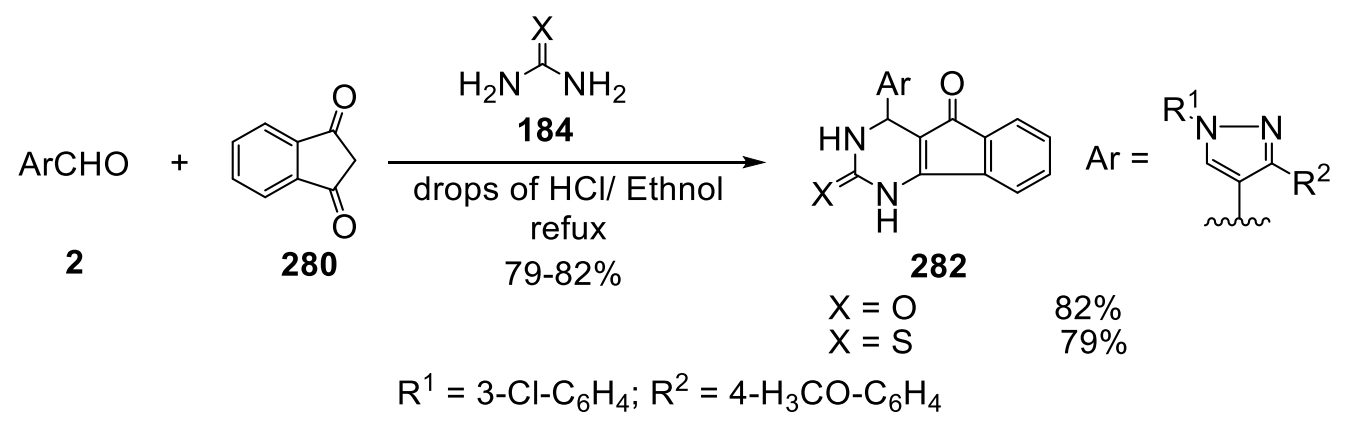

Scheme 131. Synthesis of indeno[1,2-d]pyrimidine-2-oxo (thioxo) derivatives 282.

3.2.8. Fused [6-6-6] system with one heteroatom. 3.2.2.8.1. Acridine derivatives. The reaction of pyrazole4-carboxaldehydes 2 , dimedone ${ }^{232,233}$ or 1,3-cyclohexanedione ${ }^{233} \mathbf{2 3 6}, \mathrm{NH}_{4} \mathrm{OAc} 78$ and a catalytic amount of magnetic iron oxide nanocrystals (nano $\mathrm{Fe}_{3} \mathrm{O}_{4}$ ) in a water bath under ultrasound irradiation ${ }^{232}$ or using environmentally friendly poly(4-vinylpyridinium)hydrogen sulfate $\mathrm{P}-(4-\mathrm{VPH}) \mathrm{HSO}_{4}$ as a catalyst in aqueous medium $^{233}$ afforded the corresponding 3,4,6,7,9,10-hexahydroacridine-1,8 $(2 H, 5 H)$-dione derivatives 283 (Scheme 132).

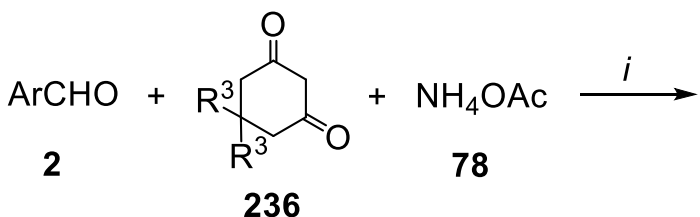

$i=$ nano $\mathrm{Fe}_{3} \mathrm{O}_{4} / \mathrm{H}_{2} \mathrm{O}$ bath )) ) irradiation; $\mathrm{P}(4-\mathrm{VPH}) \mathrm{HSO}_{4} / \mathrm{H}_{2} \mathrm{O}$, Reflux

$\mathrm{R}^{1}=\mathrm{C}_{6} \mathrm{H}_{5} ; \mathrm{R}^{2}=3-\mathrm{H}_{3} \mathrm{CO}-\mathrm{C}_{6} \mathrm{H}_{4}, 3-\mathrm{Cl}-\mathrm{C}_{6} \mathrm{H}_{4}, 4-\mathrm{O}_{2} \mathrm{~N}-\mathrm{C}_{6} \mathrm{H}_{4}, 4-\mathrm{HO}-\mathrm{C}_{6} \mathrm{H}_{4}, 4-\mathrm{Cl}-3-\left(\mathrm{H}_{5} \mathrm{C}_{6}\right)-\mathrm{C}_{6} \mathrm{H}_{3}, 3-\mathrm{H}_{3} \mathrm{C}-\mathrm{C}_{6} \mathrm{H}_{4}, \mathrm{C}_{6} \mathrm{H}_{5}, 4-\mathrm{H}_{3} \mathrm{C}-$

$\mathrm{C}_{6} \mathrm{H}_{4}, 4-\mathrm{Cl}-\mathrm{C}_{6} \mathrm{H}_{4}, 4-\left(\mathrm{H}_{5} \mathrm{C}_{6}\right)-\mathrm{C}_{6} \mathrm{H}_{4}, 4-\mathrm{H}_{3} \mathrm{CO}-\mathrm{C}_{6} \mathrm{H}_{4} ; \mathrm{R}^{3}=\mathrm{H}, \mathrm{CH}_{3}$.

Scheme 132. Synthesis of 3,4,6,7,9,10-hexahydroacridine-1,8(2H,5H)-dione derivatives 283.

3.2. 4-pyrazolyl-4H-naphthopyran derivatives 285 and 286 by one-pot three-component cyclocondensation reactions of pyrazole-4-carboxaldehydes $\mathbf{2}$, malononitrile $\mathbf{5 3}$, and naphthols $\mathbf{2 8 4 a}$ or $\mathbf{2 8 4 b}$, respectively, in the presence of piperidine as a catalyst (Scheme 133).

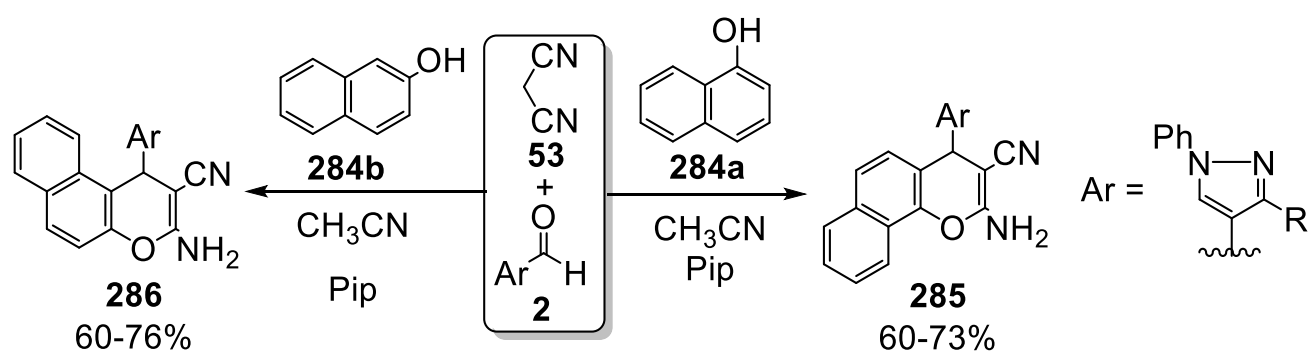

$\mathrm{R}=\mathrm{C}_{6} \mathrm{H}_{5}, 4-\mathrm{Br}-\mathrm{C}_{6} \mathrm{H}_{4}, 4-\mathrm{Cl}-\mathrm{C}_{6} \mathrm{H}_{4}, 4-\mathrm{F}-\mathrm{C}_{6} \mathrm{H}_{4}, 4-\mathrm{H}_{3} \mathrm{CO}-\mathrm{C}_{6} \mathrm{H}_{4}, 4-\mathrm{H}_{3} \mathrm{C}-\mathrm{C}_{6} \mathrm{H}_{4}, 4-\mathrm{O}_{2} \mathrm{~N}-\mathrm{C}_{6} \mathrm{H}_{4}, 2-$ Thienyl.

Scheme 133. Synthesis of 4-pyrazolyl-4H-naphthopyran derivatives 285 and 286. 
3.2.2.3. Xanthene derivatives. Neena et al. ${ }^{234}$ reported that heating of 1-phenyl-3-aryl-1H-pyrazole-4carboxaldehyde $\mathbf{2}$ with two equivalents of dimedone $\mathbf{2 3 6}$ in ethanol solution containing a catalytic amount of concentrated $\mathrm{HCl}$ at reflux afforded 3,3,6,6-tetramethyl-9-(3-aryl-1-phenyl-1H-pyrazol-4-yl)3,4,5,6,7,9hexahydro-1H-xanthene-1,8(2H)-diones 287 (Scheme 134).

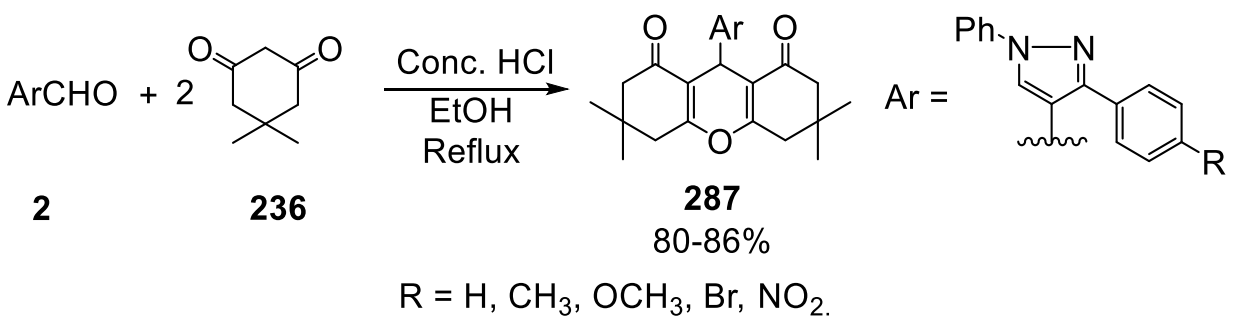

Scheme 134. Synthesis of 9-(3-aryl-1-phenyl-1H-pyrazol-4-yl)hexahydro-1H-xanthene-1,8(2H)-diones 287.

3.2.2.9. Fused [6-6-6] system with two heteroatoms. 3.2.2.9.1. Naphtho[1,2-e][1,3]oxazine derivatives. AbouElmagd and Hashem ${ }^{235}$ reported the preparation of 1-amidoalkyl-2-naphthols 288 via a one-pot condensation reaction of 1,3-diphenyl-pyrazole-4-carboxaldehyde 2, naphthalen-2-ol 284b and amides 184 in the presence of anhydrous zinc chloride under solvent-free conditions. Ring closure of $\mathbf{2 8 8}$ in ethanol at reflux gave the pyrazol4-ylnaphtho[1,2-e][1,3]oxazine derivatives 289. On the other hand, the reaction of 2-naphthol 284b with two mole equivalents of 1,3-diphenyl-pyrazole-4-carboxaldehyde 2, and ammonia solution gave 1,3-bis(1,3diphenyl-1H-pyrazol-4-yl)-2,3-dihydro-1H-naphtho[1,2-e][1,3]oxazine 290 (Scheme 135).

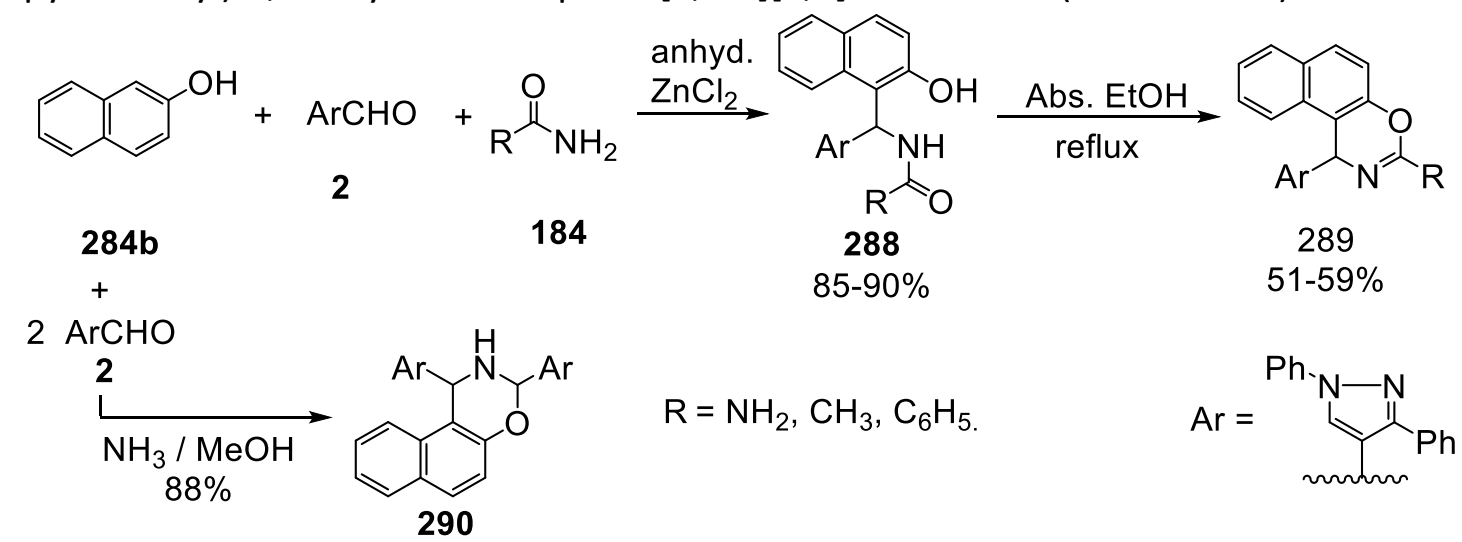

Scheme 135. Synthesis of pyrazol-4-ylnaphtho[1,2-e][1,3]oxazine derivatives 289 and 290.

3.2.2. 2 . Pyrano[3,2-c]chromene derivatives. Heating of 4-hydroxy coumarin 291 with substituted pyrazole-4carboxaldehydes $\mathbf{2}$ and malononitrile $\mathbf{5 3}$ in ethanol at reflux in the presence of piperidine as base catalyst afforded 2-amino-4- (3-(4-substituted)-1-phenyl-1H-pyrazol-4-yl)-5-oxo-4,5-dihydropyrano[3,2-c]chromene-3carbonitriles $2 \mathbf{2 9 2}^{172}$ (Scheme 136). 


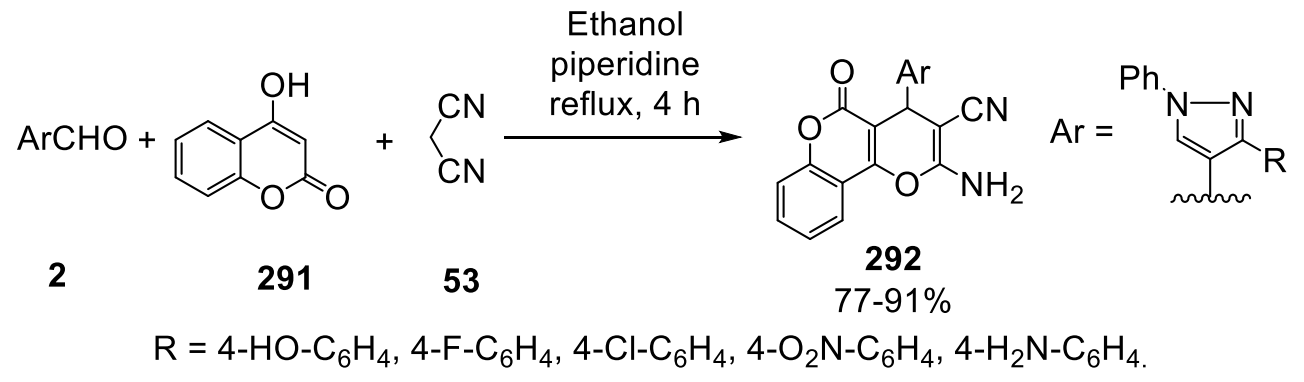

Scheme 136. Synthesis of (1H-pyrazol-4-yl)-5-oxo-4,5-dihydropyrano[3,2-c]chromene-3-carbonitriles 292.

3.2.2.9.3. Chromeno[4,3-b]pyridine derivatives. Reddy and Rao ${ }^{236}$ reported that the reaction of pyrazole-4carboxaldehydes 2 with chroman-4-one 293 gave $\alpha, 6$-unsaturated ketone system 294. Initial Michael addition of phenacylpyridinim bromide 101 with compound 294 gave 1,5-dicarbonyl system 295 which subsequently underwent cyclization in the presence of $\mathrm{NH}_{4} \mathrm{OAc} /$ acetic acid to give chromeno[4,3-b]pyridines 296 (Scheme 137).

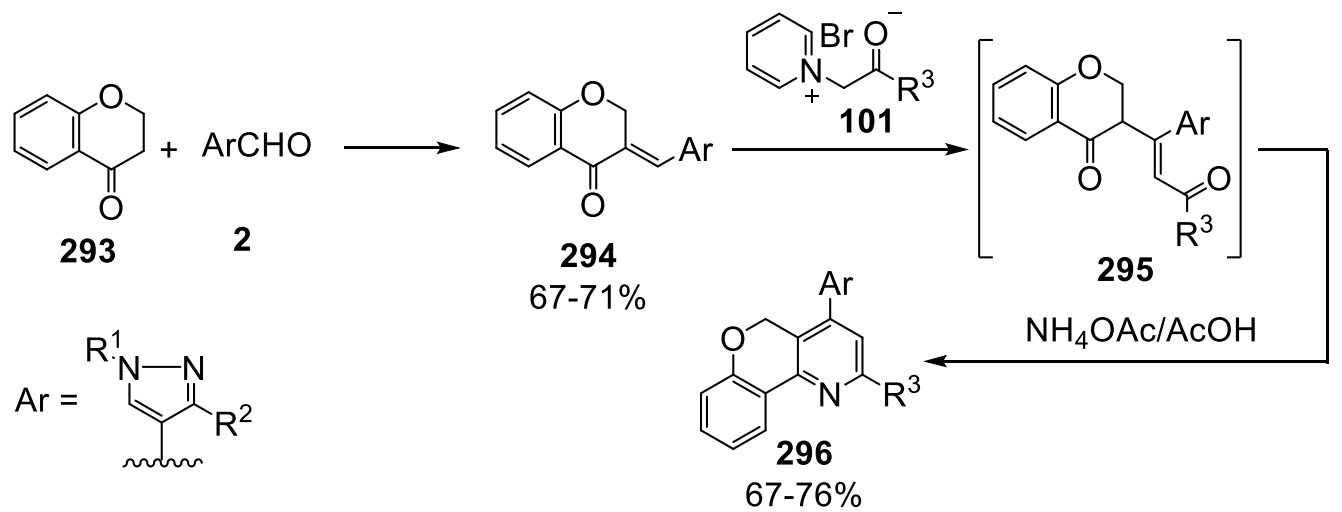

$\mathrm{R}^{1}=4-\mathrm{H}_{3} \mathrm{C}-\mathrm{C}_{6} \mathrm{H}_{4}, 4-\mathrm{F}-\mathrm{C}_{6} \mathrm{H}_{4}, 3-\mathrm{H}_{3} \mathrm{C}-\mathrm{C}_{6} \mathrm{H}_{4}, 2-\mathrm{F}-\mathrm{C}_{6} \mathrm{H}_{4} ; \mathrm{R}^{2}=4-\mathrm{Cl}_{-} \mathrm{C}_{6} \mathrm{H}_{4}, 4-\mathrm{F}-\mathrm{C}_{6} \mathrm{H}_{4}, 4-\mathrm{Br}-\mathrm{C}_{6} \mathrm{H}_{4}, 4-\mathrm{H}_{3} \mathrm{C}-\mathrm{C}_{6} \mathrm{H}_{4} ; \mathrm{R}^{3}=\mathrm{C}_{6} \mathrm{H}_{5}, 4-\mathrm{Cl}-$ $\mathrm{C}_{6} \mathrm{H}_{4}$.

Scheme 137. Synthesis of chromeno[4,3-b]pyridines 296.

3.2.2.10. Fused [6-6-6] system with three heteroatoms. 3.2.2.10.1. Thiochromeno[3,4-d]pyrimidine derivatives. Suresh et al. ${ }^{237}$ reported the synthesis of thiochromeno[3,4- $d$ ] pyrimidine derivatives 298 via a onepot three-component reaction of thiochrome-4-one 297, pyrazole-4-carboxaldehydes 2, and thiourea 184 in the presence of 1-butyl-3-methylimidazolium hydrogen sulfate [ $\mathrm{Bmim}^{\mathrm{H}} \mathrm{HSO}_{4}$ (Scheme 138).

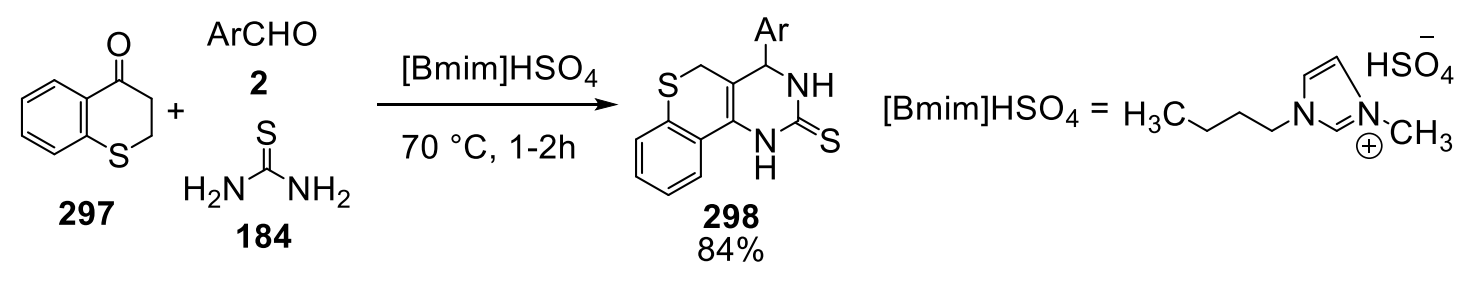

$\mathrm{Ar}=$ Pyrazole, 4- $\mathrm{O}_{2} \mathrm{~N}-\mathrm{Pyrazole}, 4-\mathrm{Cl}-\mathrm{Pyrazole}, 4-\mathrm{H}_{3} \mathrm{CO}-\mathrm{Pyrazole}$.

Scheme 138. Synthesis of thiochromeno[3,4-d]pyrimidine derivatives 298. 
3.2.2.10.2. Pyrimido[4,5-b]quinoline derivatives. Jourshari et al. ${ }^{238}$ reported that 5,8,9,10tetrahydropyrimido[4,5-b]quinoline-4,6(3H,7H)-dione derivatives 299 were synthesized by one-pot threecomponent reaction of pyrazole-4-carboxaldehydes 2, dimedone or cyclohexanedione 236 and 6-amino-2(methylthio)pyrimidin-4(3H)-one 257 in ethanol under ultrasonic irradiation in excellent yields (94-99\%) (Scheme 139).

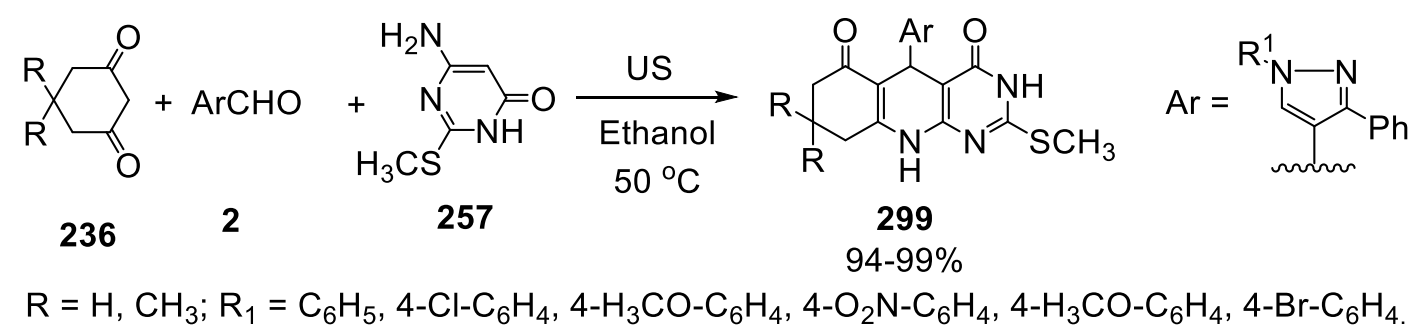

Scheme 139. Synthesis of tetrahydropyrimido[4,5-b]quinoline-4,6(3H,7H)-dione derivatives 299.

3.2.2.11. Fused [6-6-6] system with five heteroatoms. 3.2.1.2.11.1. Pyrido[2,3-d:6,5-d']dipyrimidine derivatives. Abdel-Aziem et al. ${ }^{239}$ reported that the reaction of two equivalents of 6-aminothiouracil 260 with 1,3-diphenyl-1H-pyrazole-4-carboxaldehyde 2 in methanol containing few drops of hydrochloric acid led to the formation of 5-(1,3-diphenyl-1H-pyrazol-4-yl)-2,8-dithioxo-2,3,5,8,9,10-hexahydropyrido [2,3-d:6,5d']dipyrimidine-4,6(1H,7H)-dione 300 (Scheme 140).

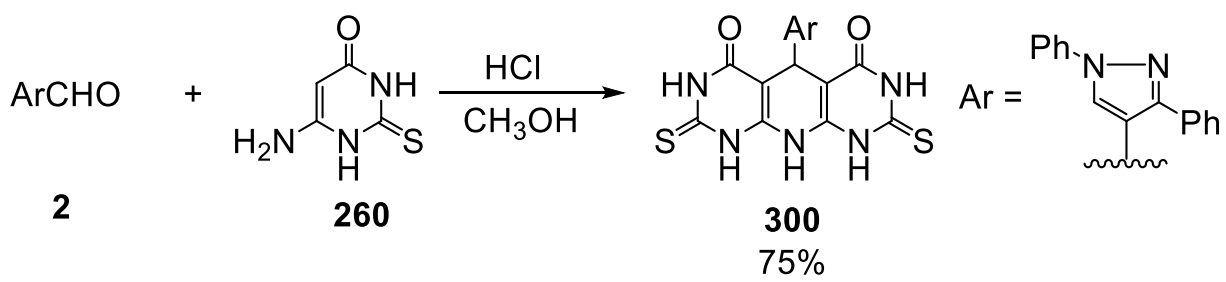

Scheme 140. Synthesis of 5-(1H-pyrazol-4-yl)hexahydropyrido [2,3-d:6,5-d']dipyrimidine-4,6(1H,7H)-dione 300 .

3.2.3. Pyrazole-substituted tetracyclic system. 3.2.3.1. Fused [6-5-6-6] system with two heteroatoms. 3.2.3.1.1. Benzo $[g]$ thieno[3,4-b]thiochromene derivatives. Metwally et al. ${ }^{209}$ reported that $[4+2]$ cycloaddition reaction of 5-pyrazolylmethylene derivatives 231 with 1,4-naphthoquinone 301 afforded benzo[g]thieno[3,4b]thiochromenes 302 (Scheme 141).

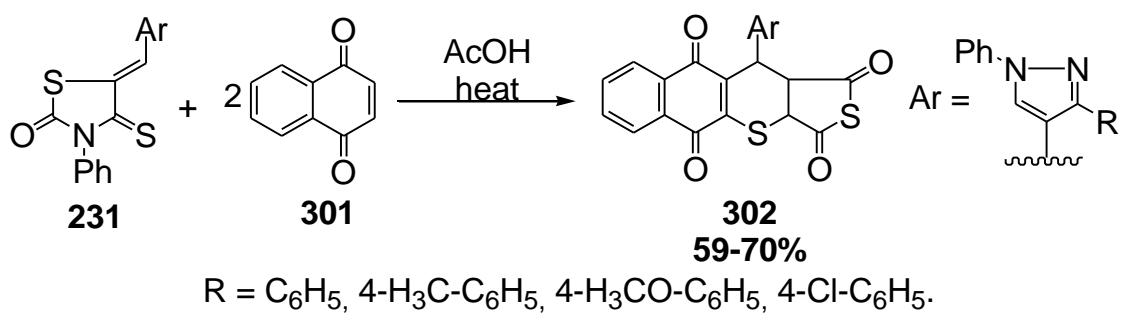

Scheme 141. Synthesis of benzo[g]thieno[3,4-b]thiochromenes 302. 
3.2.3.2. Fused [6-5-6-6] system with four heteroatoms. 3.2.3.2.1. Imidazo[4,5-f][1,10]phenanthroline derivatives. Liu et al. ${ }^{240}$ reported that heating a mixture of 1,10-phenanthroline-5,6-dione 303, ammonium acetate 78 and pyrazole-carboxaldehydes $\mathbf{2}$ in glacial acetic acid gave Imidazo[4,5-f][1,10]phenanthroline derivatives 304 (Scheme 142).

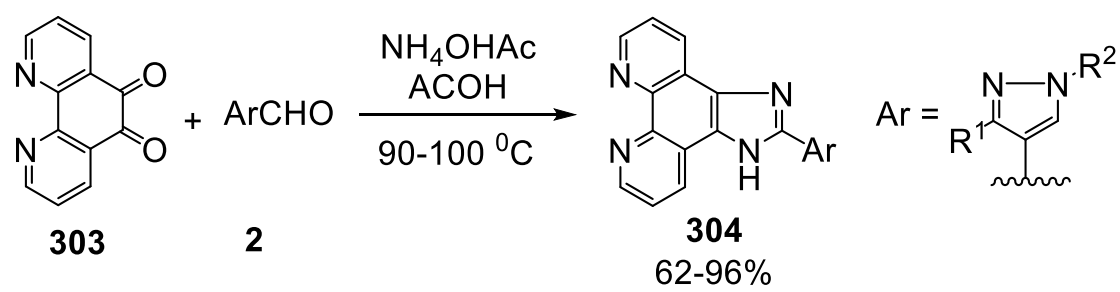

$$
\begin{gathered}
\mathrm{R}^{1}=4-\mathrm{F}-\mathrm{C}_{6} \mathrm{H}_{4}, 4-\mathrm{Cl}-\mathrm{C}_{6} \mathrm{H}_{4}, 4-\mathrm{Br}_{-}-\mathrm{C}_{6} \mathrm{H}_{4}, 4-\mathrm{H}_{3} \mathrm{C}-\mathrm{C}_{6} \mathrm{H}_{4} ; \mathrm{R}^{2}=\mathrm{H}, \mathrm{CH}_{3},-\mathrm{H}_{2} \mathrm{C}-\mathrm{C}_{6} \mathrm{H}_{5},-\left(\mathrm{H}_{2} \mathrm{C}\right)_{3}-\mathrm{N}\left(\mathrm{CH}_{3}\right)_{2}, 2,4-\mathrm{O}_{2} \mathrm{~N}-\mathrm{C}_{6} \mathrm{H}_{4}, 6- \\
\text { chloropyridazin-3-yl. }
\end{gathered}
$$

Scheme 142. Synthesis of Imidazo[4,5-f][1,10]phenanthroline derivatives 304.

3.2.4. Pyrazole-substituted pentacyclic system. 3.2.4.1. Fused [6-5-5-6-6] system with nine heteroatoms. 3.2.4.1.1. Pyrido[2,3-d:6,5-d']ditriazolopyrimidine derivatives. Abdel-Aziem et al. ${ }^{239}$ reported that the reaction of hexahydropyrido[2,3-d:6,5-d']dipyrimidine-4,6(1H,7H)-dione $\mathbf{3 0 0}$ with hydrazonoyl halides 105 in boiling chloroform gave ditriazolo[4,3-a]pyrimidin-5(1H)-one-dihydropyridine $\mathbf{3 0 5}$. Compound 305 was alternatively obtained by the reaction of ethyl-7-amino-5-oxo-1-phenyl-1,5-dihydro-[1,2,4]triazolo[4,3-a]pyrimidine-3carboxylate 306 with 1,3-diphenyl-1H-pyrazole-4-carboxaldehyde $\mathbf{2}$ in the presence of hydrochloric acid (Scheme 143).

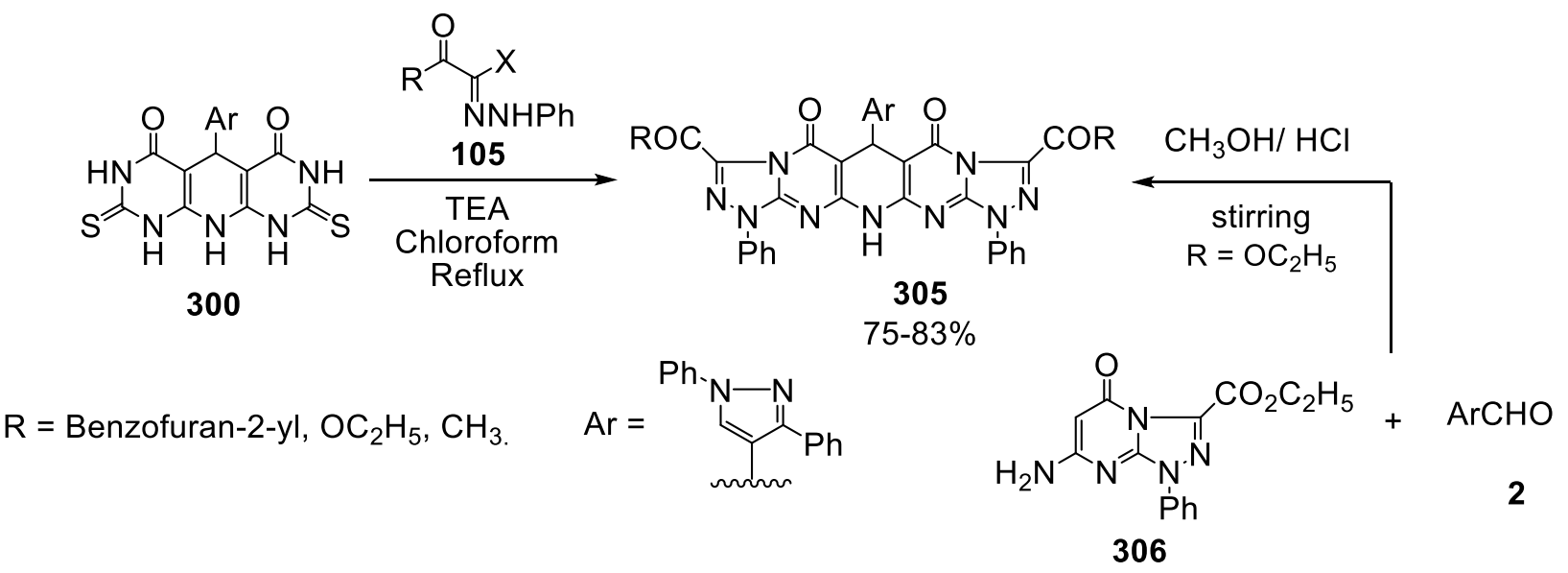

Scheme 143. Synthesis of ditriazolo[4,3-a]pyrimidin-5(1H)-one-dihydropyridines 305.

\section{Conclusions}

Heterocycles, in particular nitrogen-containing heterocycles, have been found to show a range of important applications in various fields. Among the different nitrogen-containing heterocycles, pyrazole derivatives are the most active class of five-member heterocycles due to their wide variety of important applications. This review highlighted the different synthetic methods for the preparation of pyrazole-carboxaldehydes and their 
utility as versatile precursors for various pyrazole-substituted heterocyclic systems as hybrid molecules. The heterocyclic compounds described in this review are arranged on the basis of the size of the heterocyclic ring as well as the location and number of heteroatomes. We hope that this analysis will be useful not only for synthetic organic chemists, but also for researchers interested in medicinal and biological chemistry.

\section{References}

1. Eicher, T.; Hauptmann, S.; Speicher, A. The Chemistry of Heterocycles: Structure, Reactions, Syntheses, and Applications. 2nd ed Wiley-VCH, Verlag: Weinheim, 2003.

https://doi.org/10.1002/352760183x.

2. Nylund, K.; Johansson, P.; Krutosikova, A. Heterocyclic Compounds: Synthesis, Properties and Applications Nova Science Publishers, Hauppauge: New York, 2010.

3. Bringmann, G.; Tasler, S.; Endress, H.; Mühlbacher, J. Chem. Commun. 2001, 0, 761. https://doi.org/10.1039/b101241j.

4. Y. Yamamoto Sci. Synth. Houben-Weyl Methods Mol. Transform. Six-MemberedHetarenes with Two Identical Heteroatoms 2004, 16, 714.

5. Knölker, H. J.; Reddy, K. R. Chem. Rev. 2002, 102, 4303.

https://doi.org/10.1021/cr020059j.

6. Elguero, J.; Goya, P.; Jagerovic, N.; Silva, A. M. . Em Targets in Heterocyclic Systems; Attanasi, O. A., org.; Bologna, Italy, 2002.

7. Fustero, S.; Antonio, S. F.; Sanz-Cervera, J. F. Org. Prep. Proced. Int. 2009, 41, 253. https://doi.org/10.1080/00304940903077832.

8. Fustero, S.; Sánchez-Roselló, M.; Barrio, P.; Simón-Fuentes, A. Chem. Rev. 2011, 111, 6984. https://doi.org/10.1021/cr2000459.

9. Alvarez-Builla, J.; Vaquero, J. J.; Barluenga, J. Modern Heterocyclic Chemistry, 4 Volume Set / John Wiley \& Sons. 2011.

10. Pérez-Fernández, R.; Goya, P.; Elguero, J. Arkivoc 2014, 2, 233.

https://doi.org/10.3998/ark.5550190.p008.131.

11. Karrouchi, K.; Radi, S.; Ramli, Y.; Taoufik, J.; Mabkhot, Y.; Al-aizari, F.; Ansar, M. Molecules 2018, $23,134$. https://doi.org/10.3390/molecules23010134.

12. Khan, M. F.; Alam, M. M.; Verma, G.; Akhtar, W.; Akhter, M.; Shaquiquzzaman, M. Eur. J. Med. Chem. 2016, 120, 170.

https://doi.org/10.1016/j.ejmech.2016.04.077.

13. Katritzky, A. R.; Rees, C. W. Comprehensive Heterocyclic Chemistry I Pergamon Press, Oxford, 1984.

14. Katritzky, A. R.; Rees, C. W.; Scriven, E. F. V. Comprehensive Heterocyclic Chemistry II Pergamon Press, Oxford, 1996.

15. Katritzky, A. R.; Ramsden, C. A.; Taylor, R. J. K.; Scriven, E. F. V. Comprehensive Heterocyclic Chemistry III Elsevier, 2008.

16. Katritzky, A.; Ramsden, C.; Joule, J.; Zhdankin, V. Handbook of heterocyclic chemistry Elsevier, 2010.

17. Balaban, A. T.; Oniciu, D. C.; Katritzky, A. R. Chem. Rev. 2004, 104, 2777.

https://doi.org/10.1021/CR0306790.

18. Eftekhari-Sis, B.; Zirak, M.; Akbari, A. Chem. Rev. 2013, 113, 2958.

https://doi.org/10.1021/cr300176g.

19. Ansari, A.; Ali, A.; Asif, M.; Shamsuzzaman New J. Chem. 2016, 41, 16. 
https://doi.org/10.1039/c6nj03181a.

20. Ju, Y.; Varma, R. S. J. Org. Chem. 2006, 71, 135.

https://doi.org/10.1021/jo051878h.

21. Kumari, S.; Kishore, D.; Paliwal, S.; Chauhan, R.; Dwivedi, J.; Mishra, A. Mol. Divers. 2016, $20,185$. https://doi.org/10.1007/s11030-015-9596-0.

22. Dwivedi, J.; Sharma, S.; Jain, S.; Singh, A. Mini Rev. Med. Chem. 2018, 18, 918.

https://doi.org/10.2174/1389557517666170927160919.

23. Bekhit, A. A.; Hassan, A. M. M.; Abd El Razik, H. A.; El-Miligy, M. M. M.; El-Agroudy, E. J.; Bekhit, A. E. D. A. Eur. J. Med. Chem. 2015, 94, 30. https://doi.org/10.1016/i.ejmech.2015.02.038.

24. S. Jacob, K.; G., S. Int. J. Pharm. Pharm. Sci. 2016, 8, 75. https://doi.org/10.22159/ijpps.2016v8i11.12634.

25. S. Kumar, R.; Arif, I. A.; Ahamed, A.; Idhayadhulla, A. Saudi J. Biol. Sci. 2016, 23, 614. https://doi.org/10.1016/j.sjbs.2015.07.005.

26. Alam, R.; Wahi, D.; Singh, R.; Sinha, D.; Tandon, V.; Grover, A.; Rahisuddin Bioorg. Chem. 2016, 69, 77. https://doi.org/10.1016/j.bioorg.2016.10.001.

27. Shamsuzzaman; Siddiqui, T.; Alam, M. G.; Dar, A. M. J. Saudi Chem. Soc. 2015, 19, 387. https://doi.org/10.1016/i.jscs.2012.04.009.

28. Faisal, M.; Hussain, S.; Haider, A.; Saeed, A.; Larik, F. A. Chem. Pap. 2019, 73, 1053. https://doi.org/10.1007/s11696-018-0657-6.

29. Jamwal, A.; Javed, A.; Bhardwaj, V. J. Pharm. BioSci 2013, 3, 114.

30. Fu, H. B.; Yao, J. N. J. Am. Chem. Soc. 2001, 123, 1434.

https://doi.org/10.1021/ja0026298.

31. Wang, M.; Zhang, J.; Liu, J.; Xu, C.; Ju, H. J. Lumin. 2002, 99, 79.

https://doi.org/10.1016/S0022-2313(01)00204-6.

32. Karci, F.; Karci, F.; Demirçali, A.; Yamaç, M. J. Mol. Liq. 2013, 187, 302.

https://doi.org/10.1016/j.molliq.2013.08.005.

33. Burschka, J.; Kessler, F.; Nazeeruddin, M. K.; Grätzel, M. Chem. Mater. 2013, 25, 2986.

https://doi.org/10.1021/cm400796u.

34. Kauhanka, U. M.; Kauhanka, M. M. Liq. Cryst. 2006, 33, 121.

https://doi.org/10.1080/02678290500429976.

35. Chou, P. T.; Chi, Y. Chem. - A Eur. J. 2007, 13, 380.

https://doi.org/10.1002/chem.200601272.

36. Elwahy, A.; Shaaban, M. Curr. Org. Synth. 2010, 7, 433.

https://doi.org/10.2174/157017910792246117.

37. Shaaban, M. R.; El-Sayed, R.; Elwahy, A. H. M. Tetrahedron 2011, 67, 6095.

https://doi.org/10.1016/J.TET.2011.04.096.

38. Elnagdi, M. H.; Al-Awadi, N. A.; Abdelhamid, I. A. Adv. Heterocycl. Chem. 2009, 97, 1.

https://doi.org/10.1016/S0065-2725(08)00201-8.

39. Ghozlan, S. A. S.; Abdelmoniem, A. M.; Abdelhamid, I. A. Curr. Org. Chem. 2011, 15, 3098.

https://doi.org/10.2174/138527211798357137.

40. Al-mousawi, S. M.; Sherief, M.; Abdelhamid, I. A.; Elnagdi, M. H. Curr. Org. Chem. 2011, $15,3503$.

https://doi.org/10.2174/138527211797374760.

41. Abdelmoniem, A. M.; Abdelhamid, I. A. Curr. Org. Chem. 2016, 20, 1512. 
https://doi.org/10.2174/1385272820666160216224951.

42. Ghozlan, S. A. S.; Abdelmoniem, A. M.; Ramadan, M. A.; Abdelwahab, H. M.; Gamal, M.; Abdelrahman, M.; Abdelhamid, I. A. Arkivoc 2020, 1, 297.

https://doi.org/10.24820/ark.5550190.p011.254

43. Shaaban, M. R.; Elwahy, A. H. M. Curr. Org. Synth. 2013, 10, 425.

https://doi.org/10.2174/1570179411310030007.

44. Shaaban, M. R.; Elwahy, A. H. M. Curr. Org. Synth 2014, 10, 471.

https://doi.org/10.2174/15701794113106660076.

45. Elwahy, A. H. M.; Shaaban, M. R. Curr. Org. Synth. 2014, 11, 835.

https://doi.org/10.2174/157017941106141023114039.

46. Elwahy, A. H. M.; Shaaban, M. R. Heterocycles 2017, 94, 595.

https://doi.org/10.3987/REV-16-854.

47. Abdella, A. M.; Abdelmoniem, A. M.; Abdelhamid, I. A.; Elwahy, A. H. M. J. Heterocycl. Chem. 2020, 57, 1476.

\section{https://doi.org/10.1002/jhet.3883.}

48. Riyadh, Sayed M.; Abdelhamid, Ismail Abdelshafy.; Ibrahim, Hamada M.; Al-Matar, Hamad M.; Elnagdi, M. H. Heterocycles 2007, 71, 2545.

https://doi.org/10.3987/REV-07-618.

49. Elnagdi, M. H.; Al-Awadi, N.; Abdelhamid, I. A. Comprehensive Heterocyclic Chemistry III, 10.12 - Bicyclic 5-6 Systems: Other Four Heteroatoms 2:2 Elsevier, 2008.

50. Elnagdi, M. H.; Ghozlan, S. A. S.; Abdelhamid, I. A. Arkivoc 2008, 10, 54.

https://doi.org/10.3998/ark.5550190.0009.a06

51. Riyadh, S. M.; Abdelhamid, I. A.; Al-Matar, H. M.; Hilmy, N. M.; Elnagdi, M. H. Heterocycles 2008, 75, 1849. https://doi.org/10.3987/REV-07-625.

52. Kira, M. A.; Abdel-Rahman, M. O.; Gadalla, K. Z. Tetrahedron Lett. 1969, 10, 109.

https://doi.org/10.1016/S0040-4039(01)88217-4.

53. Harikrishna, N.; Isloor, A. M.; Ananda, K.; Obaid, A.; Fun, H. K. RSC Adv. 2015, 5, 43648.

https://doi.org/10.1039/c5ra04995d.

54. Bawa, S.; Ahmad, F.; Kumar, S. Molbank 2009, 2009, M640.

https://doi.org/10.3390/m640.

55. Fun, H. K.; Arshad, S.; Malladi, S.; Selvam, R.; Isloor, A. M. Acta Crystallogr. Sect. E Struct. Reports Online 2011, 67, 245.

https://doi.org/10.1107/S1600536811023713.

56. Kumar, A.; Jain, S.; Parle, M.; Jain, N.; Kumar, P. EXCLI J. 2013, 12, 1030.

https://doi.org/10.17877/DE290R-7363.

57. Desai, N. C.; Joshi, V. V.; Rajpara, K. M.; Vaghani, H. V.; Satodiya, H. M. J. Fluor. Chem. 2012, $142,67$. https://doi.org/10.1016/j.jfluchem.2012.06.021.

58. Srikrishna, D.; Dubey, P. K. Der Pharma Chem. 2014, 6, 57.

59. Hamama, W. S.; Gouda, M. A.; Kamal El-din, H. A.; Zoorob, H. H. J. Heterocycl. Chem. 2017, $54,1369$. https://doi.org/10.1002/jhet.2716.

60. V. Laxmi, S.; S. Kuarm, B.; Rajitha, B. Med. Chem. Res. 2013, 22, 768.

https://doi.org/10.1007/s00044-012-0078-y.

61. Chornous, V. A.; Bratenko, M. K.; Vovk, M. V. Chem. Heterocycl. Compd. 2006, 42, 1242.

https://doi.org/10.1007/s10593-006-0233-9. 
62. Farghaly, A. A.; Bekhit, A. A.; Park, J. Y. Arch. Pharm. (Weinheim). 2000, 333, 53.

https://doi.org/ 10.1002/(SICI)1521-4184(200002)333:2/3<53::AID-ARDP53>3.0.CO;2-E.

63. Aruna Kumar, D. B.; Prakash, G. K.; Kumaraswamy, M. N.; Nandeshwarappa, B. P.; Sherigara, B. S.;

Mahadevan, K. M. Indian J. Chem. 2007, 46B, 336.

https://doi.org/10.1002/chin.200723094.

64. Yadlapalli, R. K.; Chourasia, O. P.; Vemuri, K.; Sritharan, M.; Perali, R. S. Bioorg. Med. Chem. Lett. 2012, $22,2708$.

https://doi.org/10.1016/j.bmcl.2012.02.101.

65. Abu-Zaied, M. A.; El-Telbani, E. M.; Elgemeie, G. H.; Nawwar, G. A. M. Eur. J. Med. Chem. 2011, 46, 229. https://doi.org/10.1016/i.ejmech.2010.11.008.

66. Matiichuk, V. S.; Potopnyk, M. A.; Obushak, N. D. Russ. J. Org. Chem. 2008, 44, 1352.

https://doi.org/10.1134/S1070428008090182.

67. Ragab, F. A.; Abdel Gawad, N. M.; Georgey, H. H.; Said, M. F. Eur. J. Med. Chem. 2013, 63, 645.

https://doi.org/10.1016/j.ejmech.2013.03.005.

68. Lebedev, A. V.; Lebedeva, A. B.; Sheludyakov, V. D.; Kovaleva, E. A.; Ustinova, O. L.; Kozhevnikov, I. B. Russ. J. Gen. Chem. 2005, 75, 782.

https://doi.org/10.1007/s11176-005-0318-7.

69. Sridhar, R.; Perumal, P. T.; Etti, S.; Shanmugam, G.; Ponnuswamy, M. N.; Prabavathy, V. R.; Mathivanan, N. Bioorg. Med. Chem. Lett. 2004, 14, 6035.

https://doi.org/10.1016/j.bmcl.2004.09.066.

70. Visagaperumal, D.; Kumar, R. J.; Vijayaraj, R.; Anbalagan, N. Int. J. ChemTech Res. 2009, 1, 1048.

71. Hu, H.; Ge, C.; Ding, L.; Zhang, A. Molecules 2010, 15, 7472.

https://doi.org/10.3390/molecules15107472.

72. Prakash, O.; Pannu, K.; Kumar, A. Molecules 2006, 11, 43.

https://doi.org/10.3390/11010043.

73. Tian, X. H.; Ma, Y. J.; Wu, X. H.; Cao, L. H. Chinese J. Org. Chem. 2007, 27, 790.

74. Prakash, O.; Pannu, K.; Naithani, R.; Kaur, H. Synth. Commun. 2006, 36, 3479.

https://doi.org/10.1080/00397910600942941.

75. Rathelot, P.; Azas, N.; El-Kashef, H.; Delmas, F.; Di Giorgio, C.; Timon-David, P.; Maldonado, J.; Vanelle, P. Eur. J. Med. Chem. 2002, 37, 671.

https://doi.org/10.1016/S0223-5234(02)01388-0.

76. Kumar, A.; Prakash, O.; Kinger, M.; Singh, S. P. Can. J. Chem. 2006, 84, 438.

https://doi.org/10.1139/V06-015.

77. Veettil, S. P.; Haridas, K. R. Molbank 2009, M624.

https://doi.org/10.3390/M624.

78. Vora, J. J.; Vasava, S. B.; Parmar, K. C.; Chauhan, S. K.; Sharma, S. S. E-Journal Chem. 2009, 6, 1205. https://doi.org/10.1155/2009/247209.

79. De Luca, L.; Giacomelli, G.; Masala, S.; Porcheddu, A. Synlett 2004, 2299.

https://doi.org/10.1055/s-2004-832809.

80. Bekhit, A. A.; Ashour, H. M. A.; Bekhit, A. E. D.; Abdel-Rahman, H. M.; Bekhit, S. A. J. Enzyme Inhib. Med. Chem. 2009, 24, 296.

https://doi.org/10.1080/14756360802188404.

81. Sharma, P. K.; Chandak, N.; Kumar, P.; Sharma, C.; Aneja, K. R. Eur. J. Med. Chem. 2011, 46, 1425. https://doi.org/10.1016/j.ejmech.2011.01.060. 
82. Meesala, R.; Nagarajan, R. Tetrahedron Lett. 2006, 47, 7557.

https://doi.org/10.1016/j.tetlet.2006.08.087.

83. Arbačiauskiene, E.; Martynaitis, V.; Krikštolaityte, S.; Holzer, W.; Šačkus, A. Arkivoc 2011, $11,1$. https://doi.org/10.3998/ark.5550190.0012.b01.

84. Barreiro, E. J.; Camara, C. A.; Verli, H.; Brazil-Más, L.; Castro, N. G.; Cintra, W. M.; Aracava, Y.; Rodrigues, C. R.; Fraga, C. A. M. J. Med. Chem. 2003, 46, 1144.

https://doi.org/10.1021/jm020391n.

85. Potapov, A. S.; Khlebnikov, A. I.; Ogorodnikov, V. D. Russ. J. Org. Chem. 2006, 42, 550. https://doi.org/10.1134/S1070428006040117.

86. Attaryan, O. S.; Antanosyan, S. K.; Panosyan, G. A.; Asratyan, G. V.; Matsoyan, S. G. Russ. J. Gen. Chem. 2006, 76, 1817.

https://doi.org/10.1134/S1070363206110260.

87. Attaryan, O. S.; Antanosyan, S. K.; Asratyan, G. V. Russ. J. Gen. Chem. 2008, 78, 508.

https://doi.org/10.1134/S1070363208030298.

88. Rstakyan, V. I.; Akopyan, A. E.; Baltayan, A. H.; Attaryan, H. S.; Asratyan, G. V. Russ. J. Gen. Chem. 2015, 85, 1191.

https://doi.org/10.1134/S1070363215050333.

89. Wallace, D. J.; Straley, J. M. J. Org. Chem. 1961, 26, 3825.

https://doi.org/10.1021/jo01068a048.

90. Robey, R. L.; Alt, C. A.; Van Meter, E. E. J. Heterocycl. Chem. 1997, 34, 413.

https://doi.org/10.1002/jhet.5570340210.

91. Zhong, W.; Chen, X.; Shen, Y. J. Chem. Res. 2010, 370.

https://doi.org/10.3184/030823410X12766918476516.

92. Babaqi, A.; El-Shekeil, A.; Hassan, M.; Shiba, S. Heterocycles 1988, 27, 2119.

https://doi.org/10.3987/COM-88-4504.

93. Aly, E.-S. A.; Abdo, M. A.; El-Gharably, A. A. J. Chinese Chem. Soc. 2004, 51, 983.

https://doi.org/10.1002/jccs.200400146.

94. Jachak, M. N.; Avhale, A. B.; Tantak, C. D.; Toche, R. B.; Reidlinger, C.; Stadlbauer, W. J. Heterocycl.

Chem. 2005, 42, 1311.

https://doi.org/10.1002/jhet.5570420710.

95. Cheng, K. M.; Huang, Y. Y.; Huang, J. J.; Kaneko, K.; Kimura, M.; Takayama, H.; Juang, S. H.; Wong, F. F. Bioorg. Med. Chem. Lett. 2010, 20, 6781.

https://doi.org/10.1016/j.bmcl.2010.08.133.

96. Nag, S.; Singh, V.; Batra, S. Arkivoc 2007, 14, 185.

https://doi.org/10.3998/ark.5550190.0008.e18

97. Ezawa, M.; Garvey, D.; Janero, D.; Khanapure, S.; Letts, L.; Martino, A.; Ranatunge, R.; Schwalb, D.;

Young, D. Lett. Drug Des. Discov. 2005, 2, 40.

https://doi.org/10.2174/1570180053398451.

98. Rodionov, A. N.; Simenel, A. A.; Korlyukov, A. A.; Kachala, V. V.; Peregudova, S. M.; Zherebker, K. Y.; Osipova, E. Y. J. Organomet. Chem. 2011, 696, 2108.

https://doi.org/10.1016/j.jorganchem.2010.11.018.

99. Kumar, R. S.; Karthikeyan, K.; Perumal, P. T. Can. J. Chem. 2008, 86, 720.

https://doi.org/10.1139/V08-059.

100. Chene, A.; Lowder, P. D.; Manning, D. T.; Newsome, P. W.; Phillips, J. L.; Ray, N. C.; Wu, T.-T. An Int. 
Appl. under Pat. Coop. 1998, WO19980282,

101. Khramchikhin, A. V; Proshkin, A. I.; Piterskaya, Y. L.; Stadnichuk, M. D. Russ. J. Gen. Chem. 1997, 67, 1816.

102. Fekri, L. Z.; Nikpassand, M.; Imani-Darestani, L. Heterocycl. Commun. 2018, 24, 151.

https://doi.org/10.1515/hc-2017-0172.

103. Abdelhamid, A. O.; A. A. Fahmi; Alsheflo, A. A. M. Int. J. Adv. Res. 2013, 1, 568.

104. Fahmy, H. H.; Khalifa, N. M.; Ismail, M. M. F.; El-Sahrawy, H. M.; Nossier, E. S. Molecules 2016, $21,1$. https://doi.org/10.3390/molecules21030271.

105. Joshi, N. S.; Shaikh, A. A.; Deshpande, A. P.; Karale, B. K.; Bhirud, S. B.; Gill, C. H. Indian J. Chem. 2005, $44 B, 422$.

106. Shelke, S. N.; Dalvi, N. R.; Gill, C. H.; Karale, B. K. Asian J. Chem. 2007, 19, 5068.

107. Bekhit, A. A.; Haimanot, T.; Hymete, A. Pak. J. Pharm. Sci. 2014, 27, 1767.

108. Alam, R.; Alam, M. A.; Panda, A. K.; Rahisuddin J. Heterocycl. Chem. 2017, 54, 1812. https://doi.org/10.1002/jhet.2768.

109. Alam, R.; Alam, A.; Panda, A. K.; Rahisuddin Med. Chem. Res. 2018, 27, 560. https://doi.org/10.1007/s00044-017-2082-8.

110. Alam, R.; Alam, M. A.; Panda, A. K.; Uddin, R. Heterocycl. Commun. 2016, 22, 221. https://doi.org/10.1515/hc-2016-0042.

111. Ramadan, E. S.; Sharshira, E. M.; El Sokkary, R. I.; Morsy, N. Zeitschrift f?r Naturforsch. B 2018, 73, 389. https://doi.org/10.1515/znb-2018-0009.

112. Abdel-Aziem, A.; Abdelhamid, A. O. Int. J. Adv. Res. 2013, 1, 717.

113. Kumar, G.; Tanwar, O.; Kumar, J.; Akhter, M.; Sharma, S.; Pillai, C. R.; Alam, M. M.; Zama, M. S. Eur. J. Med. Chem. 2018, 149, 139.

https://doi.org/10.1016/j.ejmech.2018.01.082.

114. Nossier, E. S.; Fahmy, H. H.; Khalifa, N. M.; El-Eraky, W. I.; Baset, M. A.; McPhee, D. J. Molecules 2017, 22, 512.

https://doi.org/10.3390/molecules22040512.

115. Khunt, R. C.; Khedkar, V. M.; Chawda, R. S.; Chauhan, N. A.; Parikh, A. R.; Coutinho, E. C. Bioorg. Med. Chem. Lett. 2012, 22, 666.

https://doi.org/10.1016/j.bmcl.2011.10.059.

116. Bratenko, M. K.; Chornous, V. A.; Vovk, M. V. Russ. J. Org. Chem. 2001, 37, 556.

https://doi.org/10.1023/A:1012442205046.

117. Ali, T. E. Heteroat. Chem. 2013, 24, 426.

https://doi.org/10.1002/hc.21110.

118. Fahmy, H. H.; Khalifa, N. M.; Ismail, M. M. F.; El-Sahrawy, H. M.; Nossier, E. S. Lat. Am. J. Pharm. 2016, 35, 1340.

119. Atta-Allah, S. R.; Abou-Elmagd, W. S. I.; Kandeel, K. A. A.; Hemdan, M. M.; Haneen, D. S. A.; Youssef, A. S. A. J. Chem. Res. 2017, 41, 617. https://doi.org/10.3184/174751917X15065183733150.

120. Fahmy, H. H.; Khalifa, N. M.; Nossier, E. S.; Abdalla, M. M.; Ismai, M. M. F. Acta Pol. Pharm. - Drug Res. 2012, 69, 411.

121. Youssef, A. S. A.; Hemdan, M. M.; El-Mariah, F. A.; Hashem, H. E. J. Heterocycl. Chem. 2018, 55, 1626. https://doi.org/10.1002/jhet.3197.

122. Muthineni, N.; Satish Kumar, N.; Chandrasekhara Rao, L.; Dileep Kumar, V.; Misra, S.; Raju Chowhan, L.; 
Meshram, H. M. ChemistrySelect 2016, 1, 4197.

https://doi.org/10.1002/slct.201600915.

123. Abou Elmagd, W. S. I.; Hemdan, M. M.; Samy, S. S.; Youssef, A. S. A. J. Heterocycl. Chem. 2017, 54, 1391. https://doi.org/10.1002/jhet.2719.

124. Maliszewska-Guz, A.; Wujec, M.; Pitucha, M.; Dobosz, M.; Chodkowska, A.; Jagiełło-Wójtowicz, E.;

Mazur, L.; Kozioł, A. E. Collect. Czechoslov. Chem. Commun. 2005, 70, 51.

https://doi.org/10.1135/cccc20050051.

125. Aly, E. S. A.; El-Borai, M. A.; Barren, M. A. Indian J. Chem. 2004, 43B, 1355.

https://doi.org/10.1002/chin.200440126.

126. Harras, M. F.; Sabour, R. Bioorg. Chem. 2018, 78, 149.

https://doi.org/10.1016/j.bioorg.2018.03.014.

127. Shirole, G. D.; Kadnor, V. A.; Tambe, A. S.; Shelke, S. N. Res. Chem. Intermed. 2017, 43, 1089.

https://doi.org/10.1007/s11164-016-2684-7.

128. Banothu, J.; Gali, R.; Velpula, R.; Bavantula, R. Arab. J. Chem. 2017, 10, S2754.

https://doi.org/10.1016/j.arabjc.2013.10.022.

129. Chaudhry, F.; Naureen, S.; Huma, R.; Shaukat, A.; al-Rashida, M.; Asif, N.; Ashraf, M.; Munawar, M. A.; Khan, M. A. Bioorg. Chem. 2017, 71, 102.

https://doi.org/10.1016/j.bioorg.2017.01.017.

130. B'Bhatt, H.; Sharma, S. J. Heterocycl. Chem. 2015, 52, 1126.

https://doi.org/10.1002/jhet.1992.

131. Viveka, S.; Dinesha; Madhu, L. N.; Nagaraja, G. K. Monatsh. Chem. 2015, 146, 1547.

https://doi.org/10.1007/s00706-015-1428-5.

132. D. Shirole, G.; N. Shelke, S. Lett. Org. Chem. 2016, 13, 742.

https://doi.org/10.2174/1570178614666161114165113.

133. Bekhit, A. A.; Fahmy, H. T. Y. Arch. Pharm. (Weinheim). 2003, 336, 111.

https://doi.org/10.1002/ardp.200390007.

134. Madhavilatha, B.; Fatima, N.; Sabitha, G.; Reddy, B. V. S.; Yadav, J. S.; Bhattacharjee, D.; Jain, N. Med. Chem. Res. 2017, 26, 1753.

https://doi.org/10.1007/s00044-017-1884-z.

135. Parmar, K. A.; Prajapati, S. N.; Joshi, S. A.; Goswami, K. V; Patel, A. N. Der Chem. Sin. 2011, 2, 100.

136. Bekhit, A. A.; Fahmy, H. T. Y.; Rostom, S. A. F.; Bekhit, A. E. D. A. Eur. J. Med. Chem. 2010, 45, 6027. https://doi.org/10.1016/j.ejmech.2010.10.001.

137. Tukaram, V.; Ganure, K. A.; Venkat, S.; Lohar, K. S. Heterocycl. Lett. 2017, 7, 295.

138. Taherkhorsand, H.; Nikpassand, M. Comb. Chem. High Throughput Screen. 2018, 21, 65. https://doi.org/10.2174/1386207321666180124094055.

139. Nikpassand, M.; Fekri, L. Z.; Taherkhorsand, H. Heterocycl. Commun. 2017, 23, 429. https://doi.org/10.1515/hc-2017-0124.

140. Chavan, A. S.; Kharat, A. S.; Bhosle, M. R.; Mane, R. A. Heterocycl. Commun. 2018, 24, 103. https://doi.org/10.1515/hc-2017-0130.

141. El-Emary, T. I.; Bakhite, E. A. Pharmazie 1999, 54, 106. https://doi.org/10.1002/chin.199921122.

142. Alegaon, S. G.; Hirpara, M. B.; Alagawadi, K. R.; Jalalpure, S. S.; Rasal, V. P.; Salve, P. S.; Kumbar, V. M. Med. Chem. Res. 2017, 26, 1127.

https://doi.org/10.1007/s00044-017-1821-1. 
143. Bekhit, A. A.; Ashour, H. M. A.; Bekhit, A. E.-D. A.; Bekhit, S. A. Med. Chem. (Los. Angeles). 2009, 5, 103.

144. Nossier, E. S.; El-Hallouty, S. M.; Zaki, E. R. Int J Pharm Sci 2015, 7, 353.

145. El-Bordany, E. A.; Abdel Aziz, A.; Abou-Elmagd, W. S. I.; Hashem, A. I. J. Heterocycl. Chem. 2018, 55, 291. https://doi.org/10.1002/jhet.3048.

146. Khalifa, N. M.; Nossier, E. S.; Al-Omar, M. A. Russ. J. Gen. Chem. 2017, 87, 1295. https://doi.org/10.1134/S1070363217060263.

147. Netankar, P. D.; Lingampalle, D. L.; Jagrut, V. B.; Jawale, D. V; Mane, R. A. Chem. Biol. 2012, 2, 246.

148. Nikpassand, M.; Zare Fekri, L.; Sanagou, S. Heterocycl. Commun. 2016, 22, 243. https://doi.org/10.1515/hc-2016-0024.

149. Khalifa, N. M.; Nossier, E. S.; Al-Omar, M. A. Russ. J. Gen. Chem. 2017, 87, 868. https://doi.org/10.1134/S1070363217040338.

150. Gaffer, H. E.; Abdel-Fattah, S.; Etman, H. A.; Abdel-Latif, E. J. Heterocycl. Chem. 2017, 54, 331. https://doi.org/10.1002/jhet.2588.

151. Renuka, N.; Vivek, H. K.; Pavithra, G.; Ajay Kumar, K. Russ. J. Bioorganic Chem. 2017, 43, 197. https://doi.org/10.1134/S106816201702011X.

152. Dayakar, C.; Kumar, B. S.; Sneha, G.; Sagarika, G.; Meghana, K.; Ramakrishna, S.; Prakasham, R. S.; China Raju, B. Bioorg. Med. Chem. 2017, 25, 5678.

https://doi.org/10.1016/j.bmc.2017.08.042.

153. Hawass, M. A. E.; Sanad, S. M. H.; Ahmed, A. A. hameed M.; Elneairy, M. A. E. A. E. J. Sulfur Chem. 2018, $39,388$.

https://doi.org/10.1080/17415993.2018.1435657.

154. Goudgaon, N. M.; U, S. B.; Tukaram, V. J. Pharm. Res. 2014, 8, 17.

155. Arafa, W. A. A.; Makhlouf, A. A. Int. J. Adv. Res. 2013, 1, 320.

156. Hussen, D. A.; Bekhit, A. A.; Hymete, A. Ethiop. Pharm. J. 2009, 27, 112.

https://doi.org/10.4314/epj.v27i2.58273.

157. Shejale, S. R.; Awati, S. S.; Gandhi, J. M.; Satpute, S. B.; Patil, S. S.; Kondawar, M. S. Der Pharma Chem. 2014, 6, 75.

158. Jagath Reddy, G.; Pallavi, K.; Shailaja Reddy, R.; Srinivasa Rao, K. Indian J. Chem. - Sect. B Org. Med. Chem. 2005, 44, 812.

https://doi.org/10.1002/chin.200534191.

159. Brahmbhatt, D. I.; Kaneria, A. R.; Patel, A. K.; Patel, N. H. Indian J. Chem. - Sect. B Org. Med. Chem. 2010, $49,971$.

https://doi.org/10.1002/chin.201047141.

160. Kundaliya, K. N.; Chovatiya, Y. L.; Patel, N. H.; Brahmbhatt, D. I. Der Pharma Chem. 2016, 8, 303.

161. Abdelrazek, F. M.; Michael, F. A.; Mohamed, A. E. Arch. Pharm. (Weinheim). 2006, 339, 305. https://doi.org/10.1002/ardp.200500259.

162. Hafiz, I. S. A.; Rashad, M. E. E.; Mahfouz, M. A. E.; Elnagdi, M. H. J. Chem. Res. Synopses 1998, 690.

163. Khalifa, N. M.; Al-Omar, M. A.; Taha, M. M. Russ. J. Gen. Chem. 2017, 87, 2966.

https://doi.org/10.1134/S1070363217120398.

164. Li, J. P.; Qiu, J. K.; Li, H. J.; Zhang, G. S. J. Chinese Chem. Soc. 2011, 58, 268.

https://doi.org/10.1002/jccs.201190087.

165. El-Ebiary, N. M.; Swellem, R. H.; Nawwar, G. A. M. Pharm. Chem. J. 2017, 51, 39.

https://doi.org/10.1007/s11094-017-1554-y.

166. El-Emary, T. I.; Khalil, A.; El-Hag Ali, G. A. M.; El-Adasy, A. A. A. M. Phosphorus, Sulfur Silicon Relat. Elem. 
2005, 180, 19.

https://doi.org/10.1080/10426500490494778.

167. Desai, N. C.; Rajpara, K. M.; Joshi, V. V. Bioorg. Med. Chem. Lett. 2013, 23, 2714.

https://doi.org/10.1016/j.bmcl.2013.02.077.

168. Khalifa, N. M.; Al-Omar, M. A.; Nossier, E. S. Russ. J. Gen. Chem. 2017, 87, 846.

https://doi.org/10.1134/S1070363217040296.

169. Thakrar, S.; Bavishi, A.; Bhavsar, D.; Parekh, S.; Vala, H.; Radadiya, A.; Parmar, M.; Savant, M.; Shah, A. Synth. Commun. 2012, 42, 3269.

https://doi.org/10.1080/00397911.2011.576448.

170. Thakrar, S.; Bhavsar, D.; Jain, V.; Shah, A. Chem. Biol. 2012, 2, 220.

171. Thakrar, S.; Bavishi, A.; Radadiya, A.; Vala, H.; Parekh, S.; Bhavsar, D.; Chaniyara, R.; Shah, A. J.

Heterocycl. Chem. 2014, 51, 555.

https://doi.org/10.1002/jhet.1641.

172. Thakor, T.; Savjani, J. Int. J. PharmTech Res. 2014, 6, 1397.

173. Khalifa, N. M.; Al-Omar, M. A.; Ali, O. M. Russ. J. Gen. Chem. 2017, 87, 2933.

https://doi.org/10.1134/S1070363217120349.

174. Dawane, B. S.; Konda, S. G.; Bhosle, R. B. Der Pharma Chem. 2010, 2, 251.

175. S. M. Gomha World J. Pharm. Res. 2015, 4, 26.

176. Prakash, O.; Hussain, K.; Kumar, R.; Wadhwa, D.; Sharma, C.; Aneja, K. R. Org. Med. Chem. Lett. 2011, 1, 5.

https://doi.org/10.1186/2191-2858-1-5.

177. Li, J.; Qiu, J.; Li, H.; Zhang, G. Chinese J. Chem. 2011, 29, 511.

https://doi.org/10.1002/cjoc.201190114

178. Murugan, R.; Ramamoorthy, K.; Sundarrajan, S.; Ramakrishna, S. Tetrahedron 2012, 68, 7196.

https://doi.org/10.1016/j.tet.2012.06.017.

179. Trivedi, A.; Dodiya, D.; Dholariya, B.; Kataria, V.; Bhuva, V.; Shah, V. Chem. Biol. Drug Des. $2011,78,881$. https://doi.org/10.1111/j.1747-0285.2011.01233.x.

180. Kumar, P.; Hussain, K.; Kumar, A. Curr. Chem. Lett. 2014, 3, 75.

https://doi.org/10.5267/j.ccl.2014.1.002.

181. Paplal, B.; Nagaraju, S.; Veerabhadraiah, P.; Sujatha, K.; Kanvah, S.; Vijaya Kumar, B.; Kashinath, D. RSC Adv. 2014, 4, 54168.

https://doi.org/10.1039/c4ra07708c.

182. Appna, N. R.; Raghu, K.; Maripi, S.; Garaga, S. Der Pharma Chem. 2017, 9, 50.

183. Hussain, K.; Wadhwa, D. Int. J. Org. Chem. 2014, 04, 174.

https://doi.org/10.4236/ijoc.2014.43019.

184. Trivedi, A. R.; Dodiya, D. K.; Dholariya, B. H.; Kataria, V. B.; Bhuva, V. R.; Shah, V. H. Bioorg. Med. Chem. Lett. 2011, 21, 5181.

https://doi.org/10.1016/j.bmcl.2011.07.068.

185. El-Metwally, S.; Khalil, A. K. Acta Chim. Slov 2010, 57, 941.

186. Goudgaon, N. M.; U, S. B.; Dhage, D. Heterocycl. Lett. 2012, 2, 154.

187. Trivedi, A. R.; Bhuva, V. R.; Dholariya, B. H.; Dodiya, D. K.; Kataria, V. B.; Shah, V. H. Bioorg. Med. Chem. Lett. 2010, 20, 6100.

https://doi.org/10.1016/j.bmcl.2010.08.046.

188. Reddy, G. J.; Latha, D.; Rao, K. S. Heterocycl. Commun. 2004, 10, 331. 
https://doi.org/10.1515/HC.2004.10.4-5.331.

189. Sivaprasad, G.; Peramal, P. T. J. Heterocycl. Chem. 2005, 42, 863.

https://doi.org/10.1002/jhet.5570420518.

190. Bhatt, J. D.; Chudasama, C. J.; Patel, K. D. Arch. Pharm. (Weinheim). 2017, 350, 1.

https://doi.org/10.1002/ardp.201700088.

191. Chaudhari, P. K.; Pandey, A.; Shah, V. H. Orient. J. Chem. 2010, 26, 1377.

192. Bratenko, M. K.; Chornous, V. A.; Vovk, M. V. Russ. J. Org. Chem. 2005, 41, 95.

https://doi.org/10.1007/s11178-005-0127-9.

193. Jachak, M. N.; Avhale, A. B.; Ghotekar, B. K.; Kendre, D. B.; Toche, R. B. J. Heterocycl. Chem. 2008, 45, 1221.

https://doi.org/10.1002/jhet.5570450447.

194. Sahu, S.; Sp, S. Der Pharma Chem. 2017, 9, 120.

195. Kumbar, M. N.; Kamble, R. R.; Kamble, A. A.; Salian, S. R.; Kumari, S.; Nair, R.; Kalthur, G.; Adiga, S. K.; Prasad, D. J. Int. J. Med. Chem. 2016, 2016, 1.

https://doi.org/10.1155/2016/9890630.

196. Kamal, R.; Kumar, V.; Kumar, R.; Bhardwaj, J. K.; Saraf, P.; Kumari, P.; Bhardwaj, V. Arch. Pharm.

(Weinheim). 2017, 350, 1.

https://doi.org/10.1002/ardp.201700137.

197. Bhatt, J. D.; Chudasama, C. J.; Patel, K. D. Arch. Pharm. (Weinheim). 2016, 791.

https://doi.org/10.1002/ardp.201600148.

198. Chobe, S. S.; Adole, V. A.; Deshmukh, K. P.; Pawar, T. B.; Jagdale, B. S. Sch. Res. Libr. Arch. Appl. Sci. Res. 2014, 6, 61.

199. Hamama, W. S.; El-Bana, G. G.; Shaaban, S.; Zoorob, H. H. J. Heterocycl. Chem. 2018, 55, 971. https://doi.org/10.1002/jhet.3127.

200. Bellam, M.; Gundluru, M.; Sarva, S.; Chadive, S.; Netala, V. R.; Tartte, V.; Cirandur, S. R. Chem. Heterocycl. Compd. 2017, 53, 173.

https://doi.org/10.1007/s10593-017-2036-6.

201. Kovvuri, J.; Nagaraju, B.; Kamal, A.; Srivastava, A. K. ACS Comb. Sci. 2016, 18, 644. https://doi.org/10.1021/acscombsci.6b00107.

202. Padalkar, V. S.; Borse, B. N.; Gupta, V. D.; Phatangare, K. R.; Patil, V. S.; Sekar, N. J. Heterocycl. Chem. 2016, 53, 1347.

https://doi.org/10.1002/jhet.1506.

203. Kumar, S.; Ceruso, M.; Tuccinardi, T.; Supuran, C. T.; Sharma, P. K. Bioorg. Med. Chem. 2016, $24,2907$. https://doi.org/10.1016/j.bmc.2016.04.061.

204. Reddy, T. S.; Kulhari, H.; Reddy, V. G.; Bansal, V.; Kamal, A.; Shukla, R. Eur. J. Med. Chem. 2015, $101,790$. https://doi.org/10.1016/j.ejmech.2015.07.031.

205. Madhu, C.; Hariprasad, S.; Hymavathi, A.; Ramanjaneyulu, K.; J. S. Kumar; B. v. Rao Int J Pharm Bio Sci 2016, 7, 257.

206. Dawane, B. S.; Yemul, O. S.; Chobe, S. S.; Mandawad, G. G.; Kamble, R. D.; Shinde, A. V; Kale, V. S.; Hurne, A. O.; Pawde, M. A.; Kale, M. P.; Desai, N. P.; Salgare, R. R.; Patil, M. B.; Mundhe, S. N.; Chavan, S. R. Der Pharma Chem. 2011, 3, 300.

207. Chobe, S. S.; Kamble, R. D.; Patil, S. D.; Acharya, A. P.; Hese, S. V.; Yemul, O. S.; Dawane, B. S. Med. Chem. Res. 2013, 22, 5197. https://doi.org/10.1007/s00044-013-0487-6. 
208. Thumar, N. J.; Patel, M. P. Arkivoc 2009, 13, 363. https://doi.org/10.3998/ark.5550190.0010.d30

209. Metwally, N. H.; Badawy, M. A.; Okpy, D. S. Chem. Pharm. Bull. 2015, 63, 495. https://doi.org/10.1248/cpb.c14-00885.

210. Karale, B. K.; Chavan, V. P.; Mane, A. S.; Hangarge, R. V. . G.; C. H.; Shingare, M. S. Korean J. Med. Chem. 2000, 10, 84 .

211. Prakash, O.; Kumar, R.; Parkash, V. Eur. J. Med. Chem. 2008, 43, 435. https://doi.org/10.1016/j.ejmech.2007.04.004.

212. Waghmare, A. S.; Pandit, S. S. Iran. Chem. Commun. 2015, 3, 291.

213. Pravin N. Muli, Santosh S. Chobe, Rahul D. Kamble, S. V. H.; Pratima P. Mogle, B. S. D. World J. Pharm. Res. 2014, 3, 728.

214. Janardhan, B.; Rajitha, B.; Crooks, P. A. J. Saudi Chem. Soc. 2014, 18, 722. https://doi.org/10.1016/j.jscs.2014.01.009.

215. Mamaghani, M.; Tabatabaeian, K.; Mohammadi, M.; Khorshidi, A. J. Chem. 2013, 2013, 1. https://doi.org/10.1155/2013/490972.

216. Thumar, N. J.; Patel, M. P. Arch. Pharm. Life Sci. 2011, 344, 91. https://doi.org/10.1002/ardp.201000010.

217. Thumar, N. J.; Patel, M. P. Med. Chem. Res. 2012, 21, 1751. https://doi.org/10.1007/s00044-011-9693-2.

218. Nandakumar, A.; Perumal, P. T. Org. Lett. 2013, 15, 382.

https://doi.org/10.1021/ol303326g.

219. Gupta, P.; Gupta, S.; Sachar, A.; Kour, D.; Singh, J.; Sharma, R. L. J. Heterocycl. Chem. 2010, 47, 324. https://doi.org/10.1002/jhet.282.

220. Sahu, S.; Shrivastava, S. Chem. Sci. Trans. 2016, 5, 760. https://doi.org/10.7598/cst2016.1240.

221. Wang, M.; Zhang, T. T.; Liang, Y.; Gao, J. J. Monatsh. Chem. 2012, 143, 835. https://doi.org/10.1007/s00706-011-0648-6.

222. Mehta, H. B.; Dixit, B. C.; Dixit, R. B. Chinese Chem. Lett. 2014, 25, 741. https://doi.org/10.1016/j.cclet.2014.03.015.

223. Nia, R. H.; Mamaghani, M.; Tabatabaeian, K.; Shirini, F.; Rassa, M. Acta Chim. Slov. 2013, 60, 889. http://www.ncbi.nlm.nih.gov/pubmed/24362994.

224. Suresh, L.; Sagar Vijay Kumar, P.; Poornachandra, Y.; Ganesh Kumar, C.; Chandramouli, G. V. P. Bioorg. Med. Chem. Lett. 2017, 27, 1451.

https://doi.org/10.1016/j.bmcl.2017.01.087.

225. Lipson, V. V.; Borodina, V. V.; Zemlyanaya, N. I.; Shirobokova, M. G.; Musatov, V. I.; Shishkina, S. V.; Sofronov, D. S. Russ. J. Org. Chem. 2015, 51, 697. https://doi.org/10.1134/S1070428015050206.

226. Chobe, S. S.; Mandawad, G. G.; Yemul, O. S.; Kinkar, S. S.; Dawane, B. S. Int. J. ChemTech Res. 2011, 3, 938.

227. Chobe, S. S.; Dawane, B. S.; Tumbi, K. M.; Nandekar, P. P.; Sangamwar, A. T. Bioorg. Med. Chem. Lett. 2012, 22, 7566. https://doi.org/10.1016/j.bmcl.2012.10.027.

228. Salman, A. S. J. Chem. Biol. Phys. Sci. 2016, 6, 469.

229. Ashok, D.; Rangu, K.; Gundu, S.; Rao, V. H. Chem. Heterocycl. Compd. 2016, 52, 928. 


\section{https://doi.org/10.1007/s10593-017-1987-y.}

230. Shaikh, M. A.; Farooqui, M.; Abed, S. Res. Chem. Intermed. 2018, 44, 5483.

https://doi.org/10.1007/s11164-018-3435-8.

231. Kamal, A.; Babu, K. S.; Hussaini, S. M. A.; Srikanth, P. S.; Balakrishna, M.; Alarifi, A. Tetrahedron Lett. 2015, 56, 4619.

\section{https://doi.org/10.1016/j.tetlet.2015.06.006.}

232. Fekri, L. Z.; Nikpassand, M.; Pour, K. H. Curr. Org. Synth. 2015, 12, 76.

233. Velpula, R.; Banothu, J.; Bavantula, R. Biolological Med. Chem. Effic. eco-friendly Protoc. Synth. 2013, 1, 32.

234. Neena; Nain, S.; Bhardwaj, V.; Kumar, R. Pharm. Chem. J. 2015, 49, 254. https://doi.org/10.1007/s11094-015-1266-0.

235. Abou-Elmagd, W. S. I.; Hashem, A. I. Med. Chem. Res. 2013, 22, 2005. https://doi.org/10.1007/s00044-012-0205-9.

236. Reddy, G. J.; Rao, K. S.; Khalilullah, M.; Latha, D.; Thirupathaiah, C. Heterocycl. Commun. 2005, 11, 331.

237. Suresh, L.; Sagar Vijay Kumar, P.; Poornachandra, Y.; Ganesh Kumar, C.; Chandramouli, G. V. P. Bioorg. Chem. 2016, 68, 159.

https://doi.org/10.1016/j.bioorg.2016.08.006.

238. Jourshari, M. S.; Mamaghani, M.; Tabatabaeian, K.; Shirini, F.; Rassa, M.; Langhari, H. Lett. Org. Chem. 2012, 9, 664 .

https://doi.org/10.2174/157017812803521207.

239. Abdel-Aziem, A.; El-Gendy, M. S.; Abdelhamid, A. O. Eur. J. Chem. 2012, 3, 455. https://doi.org/10.5155/eurjchem.3.4.455-460.683.

240. Liu, J.; Chen, M.; Wang, Y.; Zhao, X.; Wang, S.; Wu, Y.; Zhang, W. Eur. J. Med. Chem. 2017, $133,36$. https://doi.org/10.1016/j.ejmech.2017.03.030.

\section{Authors' Biographies}

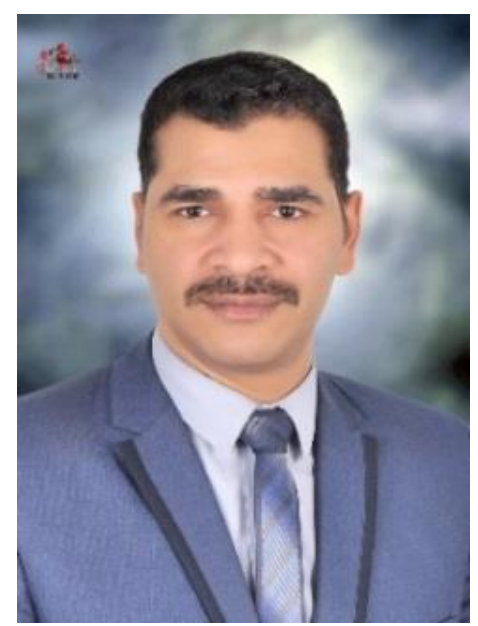

Ismail A. Abdelhamid was born in Egypt in December 1978. He graduated from Cairo University, Egypt in 2001 then he got his M.Sc. and Ph.D. degrees in 2005 and 2007, respectively, at Cairo University in the field of organic synthesis. In 2017 he was appointed as a full Professor of Organic chemistry at Cairo University. He was awarded the Alexander von Humboldt research fellowship in 2008-2011 and in 2014, 2017, and 2019 with Prof. Holger 
Butenschön, at Hannover University, Germany. He received several research prizes; Cairo University Incentive Award (2012), Cairo University Scientific Excellence Award (2016) and State Incentive Award (2019).

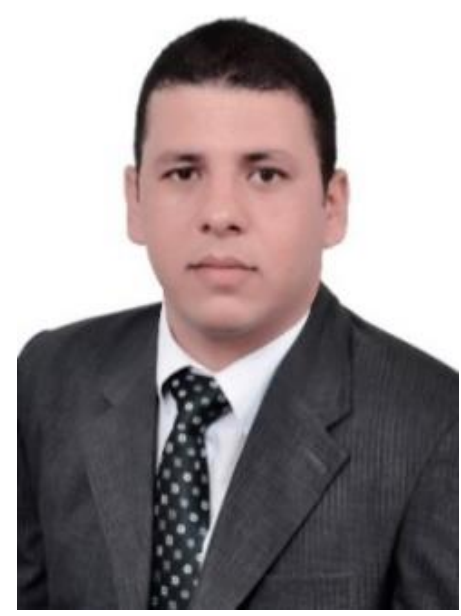

Mahmoud A. E. Hawass was born in 1985 in Giza, Egypt. He has graduated from Cairo University, Faculty of Science, Egypt in 2008 then he got his M.Sc degree in 2014. He has published four paper in the field of organic synthesis.

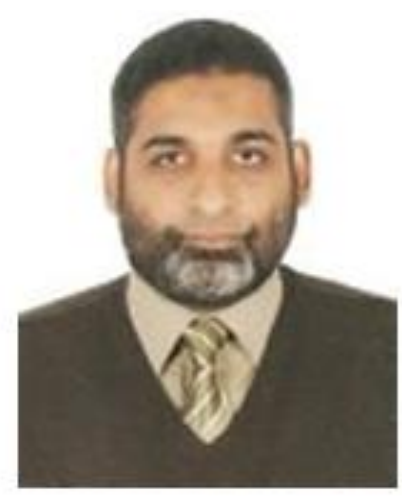

Sherif M. H. Sanad was born in Egypt in July 1980. He graduated from Cairo University, Egypt in 2002 then he got his M.Sc. and Ph.D. degrees in 2009 and 2012, respectively, at Cairo University in the field of organic synthesis. In 2019 he was appointed as associated professor of Organic chemistry at Cairo University.

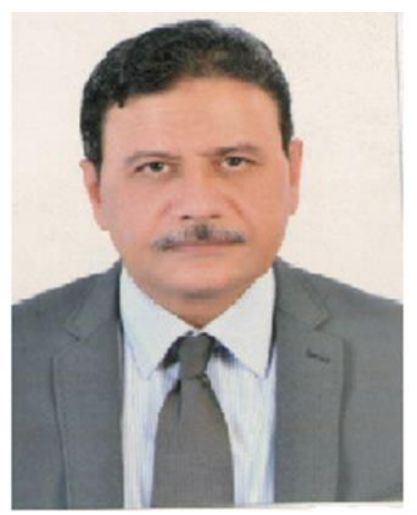

Ahmed H. M. Elwahy was born in 1963 in Giza, Egypt. He graduated from Cairo University, Egypt in 1984 then he got his M.Sc. and Ph.D. degrees in 1988 and 1991, respectively, at Cairo University in the field of organic 
synthesis. He was awarded the Alexander von Humboldt research fellowship in 1998-2000 and in 2003, 2005, 2009, 2010 and 2012 with Prof. Klaus Hafner, at TU Darmstadt, Germany. In 2002 he was appointed as a full Professor of Organic chemistry at Cairo University. In 2001 he received the State-Award in Chemistry and in 2016 received Cairo University Appreciation-Award in Basic Science. He published around 140 scientific papers in distinguished international journals.

This paper is an open access article distributed under the terms of the Creative Commons Attribution (CC BY) license (http://creativecommons.org/licenses/by/4.0/) 